\begin{tabular}{|l|l|}
\hline 2. To: (Receiving Organization) & 3. From: (Originating Organization) \\
W-030 TEST REVIEW BOARD & DISPOSAL PROJECTS \\
\hline 5. Proj./Prog./Dept./Div.: & 6. Design Authority/Design Agent/Cog. \\
PROJECT W-030 & Engr.: D.B. Cole/W.M. Harty/ \\
\hline
\end{tabular}

8. Originator Remarks:

Release of test report for pre-operational test of $\mathrm{W}-030$ Recirculation Condenser Cooling System. Project W-030 provides the $A Y / A Z$ tank farms ventilation upgrade.

11. Receiver Remarks: 11A. Design Baseline Document? [] Yes [X] No
4. Related EDT No.: 616328

7. Purchase order No.:

NA

9. Equip./Component No.:

NA

10. System/Bldg./Facility: AY/AZ Tank Farms

12. Major Assm. Dwg. No.:

NA

13. Permit/Permit Application No.: NA

14. Required Response Date: Immediate

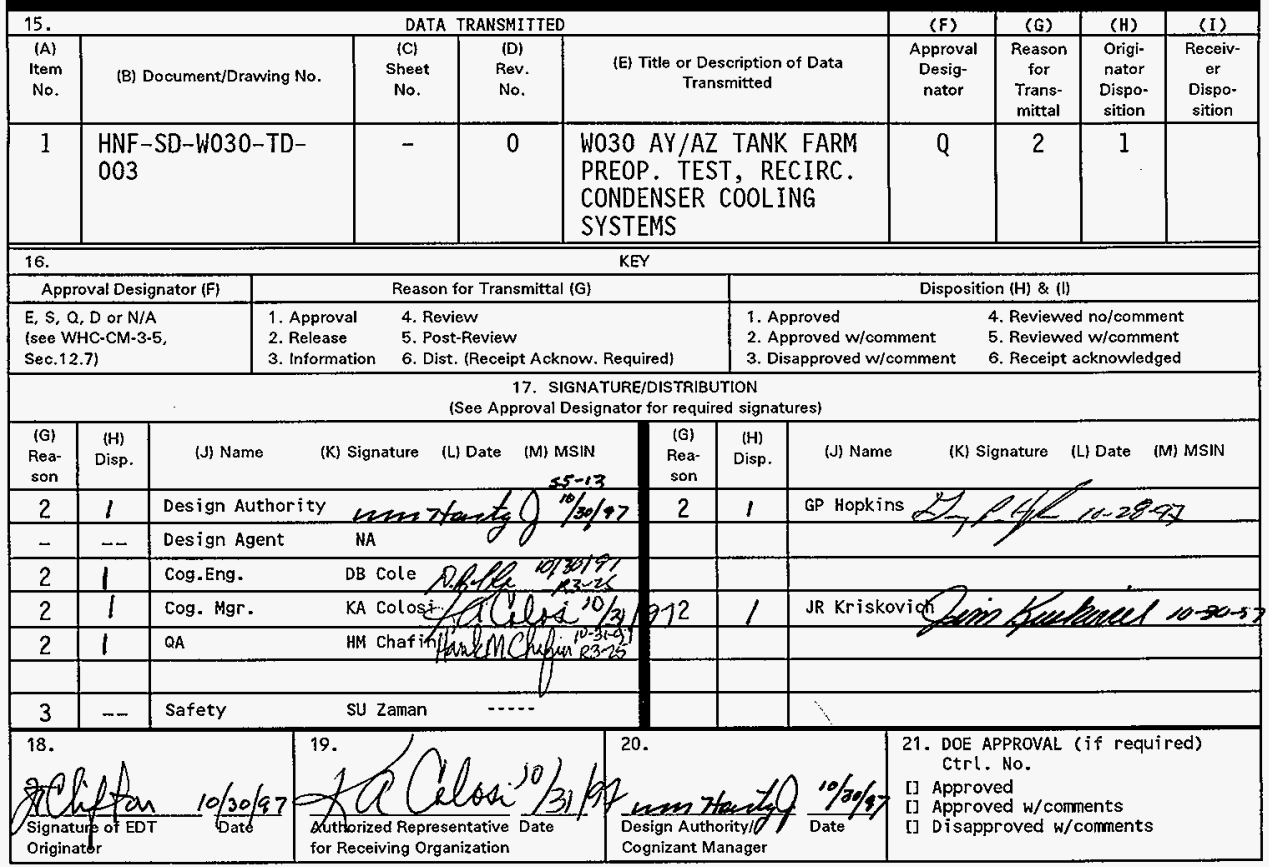

BD - 7400-172-2 (05/96) GEF097 
HNF-SD-W030-TD-003, Rev. 0

\title{
PREOPERATIONAL TEST REPORT, RECIRCULATION CONDENSER COOLING SYSTEMS
}

\author{
FT CLIFTON
}

NUMATEC HANFORD COMPANY, Richland, WA 99352

U.S. Department of Energy Contract DE-AC06-96RL13200

$\begin{array}{llll}\text { EDT/ECN: } & 622919 & \text { UC: } & 2030 \\ \text { Org Code: } & 8 C 473 & \text { Charge Code: } & \text { NH107 } \\ \text { B\&R Code: } & \text { EW3130010 } & \text { Total Pages: } 302\end{array}$

Key Words: TEST, COOLING, VENTILATION, RECIRCULATION, UPGRADE

Abstract: Preoperational test report for Recirculation Condenser Systems, Project $W-030$. Project $W-030$ provides a ventilation upgrade for the four Aging Waste Facility tanks.

The four system provide condenser cooling water for vapor space cooling of tanks AY101, AY102, AZ101, AZ102. Each system consists of a valved piping loop, a pair of redundant recirculation pumps, a closed-1oop evaporative cooling tower, and supporting instrumentation; equipment is located outside the farm on concrete slabs. Piping is routed to the each ventilation condenser inside the farm via below-grade concrete trenches. The tests verify correct system operation and correct indications displayed by the central Monitor and Control System.

TRADEMARK DISCLAIMER. Reference herein to any specific commercial product, process, or service by trade name, trademark, manufacturer, or otherwise, does not necessarily constitute or imply its endorsement, recomendation, or favoring by the United States Government or any agency thereof or its contractors or subcontractors.

Printed in the United States of America. To obtain copies of this document, contact: Document Control Services, P.O. Box 950, Mailstop H6-08, Richland WA 99352, Phone (509) 372-2420; Fax (509) 376-4989.
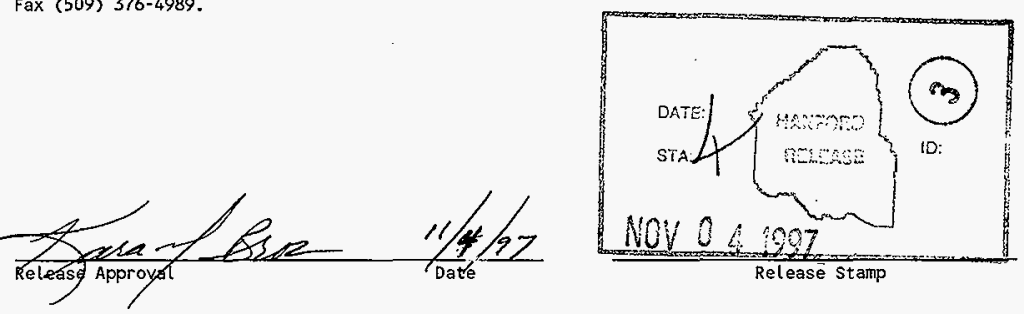

Approved for Public Release 
HNF-SD-W030-TD-003,

REV. 0, PAGE 1

\section{W030 AY/AZ TANK FARM PREOPERATIONAL TESTING, RECIRCULATION CONDENSER COOLING (EW) SYSTEM TEST REPORT}

\section{ATTACHMENTS}

Attachment 1 - Test Report Checklist

Attachment 2 - Copy of original test procedure with test review board approval sheet, recorded data, and attached sections for 4 tanks (AY101, AY102, AZ101, \& AZ102), each including the following appendices:

Appendix A - List of Instrumentation Requiring Calibration Verification Appendix B - Valve Alignment Table

Appendix C - Electrical Alignment TabTe

Appendix D - Operation Data Sheets

Appendix E - Signature/Initial Verification Table

Appendix F - Test Log

Appendix G - Test Exception Report \#W030-TE-006

Appendix $H$ - Test Exception Log

Attachment 3 - Miscellaneous Supporting Data, including:

CVI list

Relevant vendor data

Report of 4 Aug 96, M. Jennings to G. Howe17, RE: W030 Cooling System Component Vibration Data

Report of 13 Oct 97 , M. Jennings to F. Clifton, RE: W030 Cooling System Component Vibration Data [Followup Report]

Lab test reports of cooling water glycol concentration

Procedure validation checksheet

Attachment 4 - Calibration, Grooming, and ATignment (CGA) Plan, with data.

\section{REFERENCES}

1. WHC-SD-W030-POTP-003, Rev. 0

Preoperational Test Procedure,

2. WHC-SD-W030-SUP-003, Rev. 0

Recirculation Condenser Cooling System

3. $H-2-131067$

4. $\mathrm{H}-2-131068$

5. $\mathrm{H}-2-131069$

6. $\mathrm{H}-2-131070$

7. WHC-SD-W030-ATR-004, Rev. 0

Startup Test Plan, W-030

P\&ID AY101EW Recirc Condenser Cooling

P\&ID AY102EW Recirc Condenser Cooling

P\&ID AZ101EW Recirc Condenser Cooling

P\&ID AZ102EW Recirc Condenser Cooling

W-030 Acceptance Test Report, Cooling and

Miscellaneous Instrumentation

8. HNF-SD-W030-TD-004, Rev. 0

Preoperational Test Report, Primary Vent

Condenser Cooling System

\section{INTRODUCTION}

Preoperational Test WHC-SD-W030-P0TP-003 was satisfactori7y performed, starting in May 1996, with one test exception. A copy of test exception report \#W030-TE-006 is attached to this test report. This test was performed over a two month period to allow for completion of instrument calibration, loop checks, and implementation of design changes. 
The tested system includes four recirculation water-glycol cooling loops, each including a redundant pair of recirculation pumps, an evaporative cooling tower (also referred to as a "fluid cooler" in vendor literature), interconnecting valved piping, and related instrumentation. All recirculation system interlocks, alarms, overrides, and controls were tested for each system. Each system operated as designed and within expected parameters and setpoints. ATl test actions were initiated or verified at the central control room graphic screens of the Monitor and Control System (MCS).

All system instrumentation was calibrated and all system instrument loop checks were completed prior to testing in accordance with a Project W030 internal Calibration, Grooming, and Alignment (CGA) Plan. Data is included in Attachment-4. All system valves were checked for proper alignment, prior to performance of this test. The testing satisfactority met the test objectives as outlined in reference-2 for the applicable system which is designed as shown on references -3 through -6 . Performance data were collected primarily for information only, with minimal acceptance criteria. One test exception was recorded and resolved. This test report includes a copy of the final approved test procedure (reference-1), annotated with field test data and initialed by the Test Engineer.

SUMMARY OF TEST RESULTS

Acceptance criteria: The following criteria were evaluated for each of the 4 tanks, or systems, in the process of testing:

\begin{tabular}{|c|c|c|}
\hline ITEM/FUNCTION TESTED & ACCEPTANCE CRITERIA & TEST RESULT \\
\hline RECIRCULATION PUMPS & $\begin{array}{l}\text { PUMPS ARE OPERABLE AND PERFORM } \\
\text { REASONABLY CLOSE TO VENDOR DATA IN } \\
\text { BOTH FLOW AND NO-FLOW CONDITIONS }\end{array}$ & CRITERIA MET \\
\hline RECIRC PUMP VIBRATION & $\begin{array}{l}\text { VIBRATION IS TYPICAL FOR PUMPS OF THIS } \\
\text { TYPE }\end{array}$ & $\begin{array}{l}\text { CRITERIA MET; SEE } \\
\text { FOLLOWUP VIBRATION REPORT }\end{array}$ \\
\hline $\begin{array}{l}\text { RECIRC PUMP CONTROLS AND } \\
\text { INTERLOCKS }\end{array}$ & $\begin{array}{l}\text { PUMPS CAN BE CONTROLLED REMOTELY FROM } \\
\text { MCS; PUMP CONTROLS AND INTERLOCKS } \\
\text { OPERATE CORRECTLY PER DESIGN }\end{array}$ & CRITERIA MET \\
\hline $\begin{array}{l}\text { RECIRC PUMP LOW FLOW ALARM } \\
\text { (LOCATED ON RETURN LINE FROM } \\
\text { CONDENSER) }\end{array}$ & $\begin{array}{l}\text { ALARM ACTIVATES ON MCS GRAPHICS } \\
\text { SCREEN, RESETS ON RETURN TO NORMAL } \\
\text { CONDITION }\end{array}$ & CRITERIA MET \\
\hline $\begin{array}{l}\text { RECIRC PUMP LOW FLOW SHUTDOWN } \\
\text { OVERRIDE SWITCHES }\end{array}$ & SWITCHES OPERATE CORRECTLY PER DESIGN & CRITERIA MET \\
\hline $\begin{array}{l}\text { EVAPORATIVE COOLING TOWER SPRAY } \\
\text { PUMP }\end{array}$ & $\begin{array}{l}\text { PUMP IS OPERABLE AND CAN BE CONTROLLED } \\
\text { REMOTELY FROM MCS; VIBRATION IS } \\
\text { TYPICAL FOR TYPE OF PUMP }\end{array}$ & CRITERIA MET \\
\hline $\begin{array}{l}\text { EVAPORATIVE COOLING TOWER FAN AND } \\
\text { TEMPERATURE CONTROL DAMPER }\end{array}$ & $\begin{array}{l}\text { FAN IS OPERABLE AND CAN BE CONTROLLED } \\
\text { REMOTELY FROM MCS; VIBRATION IS } \\
\text { TYPICAL FOR TYPE OF FAN; DAMPER } \\
\text { MODULATES TO MAINTAIN GLYCOL TEMP. AT } \\
\text { SETPOINT }\end{array}$ & CRIIERIA MET; SEE TEXT \\
\hline $\begin{array}{l}\text { EVAPORATIVE COOLING TOWER PAN } \\
\text { HEATER }\end{array}$ & HEATER IS OPERABLE & CRITERIA MET \\
\hline
\end{tabular}


HNF-SD-W030-TD-003,

REV. 0, PAGE 3

\begin{tabular}{||l|l|c||}
\hline \multicolumn{1}{|c|}{ ITEM/FUNCTION TESTED } & \multicolumn{1}{|c|}{ ACCEPTANCE CRITERIA } & TEST RESULT \\
\hline \hline $\begin{array}{l}\text { EVAPORATIVE COOLING TOWER PAN } \\
\text { LIQUID LEVEL SWITCH }\end{array}$ & $\begin{array}{l}\text { SWITCH IS OPERABLE; INTERLOCKS WITH } \\
\text { PAN HEATER, SPRAY PUMP, COOLING FAN, } \\
\text { AND MAKEUP VALVE ARE ALL FUNCTIONAL }\end{array}$ & CRITERIA MET \\
\hline EVAPORATIVE COOLING TOWER FILTER & $\begin{array}{l}\text { PUMP IS OPERABLE, AND VIBRAIION IS } \\
\text { TYPICAL FOR TYPE OF PUMP; SYSTEM } \\
\text { RECIRC PUMP AND FILTRATION SYSTEM }\end{array}$ & \multicolumn{1}{|c|}{ CRITERIA MET; SEE } \\
& $\begin{array}{l}\text { PP INDICATION/ALARM, CONDUCTIVITY ' } \\
\text { INDICATION, AND ALL SYSTEM CONTROLS } \\
\text { AND INTERLOCKS }\end{array}$ & FOLLOWUP VIBRATION REPORT \\
\hline
\end{tabular}

Water System Filling: All four of the cooling water recirculation (EW) systems, $A Y-101, A Y-102, A Z-101$ and $A Z-102$, were turned over to $W-030$ Project Test Engineering Personnel prior to being filled with a mixture of water and propylene glycol. Sanitary water was used for filling and flushing the system and was supplied by tanker truck; raw water which is piped to the w030 site is not sufficiently pure for this service.

Prior to filling operations, a vent valve was installed at each evaporative cooling tower heat exchanger inlet and at each recirculation condenser inlet to ensure removal of all trapped air from the piping system. The four EW systems were filled using a portable engine-driven pump with a capacity of approximately $30 \mathrm{gal} / \mathrm{min}$ and a discharge head of $40 \mathrm{psig}$. Each system was flushed to remove discoloration of the water due to corrosion products in the piping, then drained in preparation for glycol addition. For freezeprotection, several 55-gallon drums of propylene glycol (DOWFROST heat transfer fluid) were added to each system to achieve a minimum concentration of $40 \%$ by volume. Copies of lab analyses are attached, showing the final glycol concentration; specific data is shown in the table below, including that used to calculate the amount of glycol needed for each system.

\begin{tabular}{||c|c|c|c||}
\hline EW SYSTEM 1.D. & $\begin{array}{c}\text { CALCULATED SYSTEM } \\
\text { VOLUME, GALLONS }\end{array}$ & GLYCOL VOLUME ADDED & $\begin{array}{c}\text { FINAL GLYCOL CONCENTRATION BY } \\
\text { LAB ANALYSIS, \% BY VOLUME }\end{array}$ \\
\hline \hline AY101 & 495 & 4.5 drums & 49 \\
\hline AY102 & 585 & 5 drums & 43 \\
\hline AZ101 & 670 & 6 drums & 42 \\
\hline AZ102 & 380 & 3.5 drums & 53 \\
\hline
\end{tabular}

The EW systems were filled as they became available and each recirculation pump was operated for minimum total of 24 hours to achieve adequate mixing of the glycol solution.

A sample was taken from each system and analyzed by the WSCF Laboratory to determine precise glycol concentration. The DOWFROST dilution water chemistry requirements are as follows: chlorides $\leq 25 \mathrm{ppm}$, sulfates $\leq 25 \mathrm{ppm}$, calcium $\leq 50 \mathrm{ppm}$, and magnesium $\leq 50 \mathrm{ppm}$. It was determined before filling that site sanitary water meets these requirements. See reference- 8 for additional manufacturer's data on DOWFROST propylene glycol heat transfer fluid. 
HNF-SD-W030-TD-003, REV. 0, PAGE 4

Pump Testing: The EW systems were the first part of each tanks recirculation cooling system to be tested. As instrument calibrations and loop checks were completed, the sections of the test procedure for the EW systems were performed.

It was noted that the mechanical seals on all of the recirculation pumps started to leak during operation of the pumps, some almost immediately and others after days of operation. It was surmised that the leaks may have resulted from construction leak testing having been performed using compressed air (in lieu of water). During testing, air was seen leaking from the seals; this air flow may have contaminated the seais with particulate matter (grit) and caused the seal failures later in the test program. Additionally, the pump shafts were found to have been painted in the sealing area, which could have contributed to the problem. Corrective maintenance is in progress on the pumps, and most of the eight recirculation pumps have been successfully overhauled and retested.

Both redundant recirculation pumps in each system were operated and determined to be operating within the ijmits of the vendor pump curve. Copies of the pump curves are attached. The following table summarizes the data recorded during the test of each recirculation pump at shutoff head and at system design flow rates.

\begin{tabular}{|c|c|c|c|c|}
\hline PUMP I.D. & PUMP FLOW， GAL/MIN & $\begin{array}{c}\text { SUCTION PRESSURE, } \\
\text { PSIG }\end{array}$ & $\begin{array}{c}\text { DISCHARGE PRESSURE, } \\
\text { PSIG }\end{array}$ & $\begin{array}{l}\text { MOTOR CURRENT, } \\
\text { AMPS }\end{array}$ \\
\hline \multirow[t]{2}{*}{ AY101-EW-P-1A } & [No $\mathrm{Flow}]$ & 13 & 60 & 7.4 \\
\hline & 192 & 11.5 & 58 & 9.5 \\
\hline \multirow[t]{2}{*}{ AY101-EW-P-1B } & [No Flow] & 13 & 52 & 7.0 \\
\hline & 192 & 11.5 & 48 & 9.4 \\
\hline \multirow[t]{2}{*}{ AY102-EW-P-1A } & [No Flow] & 8 & 47 & 7.1 \\
\hline & 195 & 6 & 42 & 9.5 \\
\hline \multirow[t]{2}{*}{ AY102-EW-P-1B } & [No $F$ low] & 7.5 & 46 & 6.9 \\
\hline & 193 & 6 & 42 & 9.4 \\
\hline \multirow[t]{2}{*}{ AZ101-EW-P-1A } & [No Flow] & 10 & 68 & 13.0 \\
\hline & 328 & 5 & 61 & 19.5 \\
\hline \multirow[t]{2}{*}{$A Z 101-E W-P-1 B$} & [No Flow] & 10 & 68 & 13.0 \\
\hline & 332 & 5 & 61 & 21.0 \\
\hline \multirow[t]{2}{*}{ AZ102-EW-P-IA } & [No Flow] & 8 & 46 & 7.3 \\
\hline & 192 & 5 & 40 & 9.5 \\
\hline \multirow[t]{2}{*}{ AZ102-EW-P-IB } & [No Flow] & 6 & 44 & 7.1 \\
\hline & 201 & 4 & 39 & 9.5 \\
\hline
\end{tabular}


Evaporative Cooling Tower Problems and Modifications: Prior to operation and testing of the evaporative cooling towers, an effort was made to flush out the tower pans to remove dirt and debris. During this effort, two design flaws were discovered and corrected:

- Solenoid operated valves, providing raw water makeup to the evaporative cooling towers, failed to operate.

- Solenoid operated valves, provided for automatic high conductivity blowdown drains, failed to operate.

Investigations and corrective actions were as follows:

- The 1-inch pipe supplying raw water to AY102 evaporative cooling tower was found to be plugged with debris (sand, pebbles, trash). Thus, the failure of the raw water makeup solenoid valves was likely due to fouling by debris in the raw water. All the valves were replaced by solenoid valves of a design more tolerant of operation in a "dirty" system. The plugged piping was back-flushed to remove the obstruction, and all supply lines to the remaining systems were flushed to ensure they were also free of debris. To preclude future problems with fouling of the makeup valves, strainers were installed in the supply piping to each system. The strainer baskets incorporate an 80-mesh screen which can be blown down when dirty.

- It was determined from vendor information that the installed solenoidoperated blowdown valves required a differential pressure of 5 psid to operate, whereas the maximum differential applied in this configuration would be less than 1 psid. Thus, they were replaced with motor-operated ball valves (MOVs) not dependent on operating pressures. Installation of the MOVs for the high-conductivity drain valves required that the control power transformer for the evaporative cooling tower be changed as well. The existing transformer was too small to carry the additiona] current load of the Mov. After the installation of the 1 kva transformers, the conductivity loops and the MOVs were tested for proper operation.

During testing, another design $\mathrm{fl}$ aw was discovered and corrected: it was noted that the spray pump and fan were tripped off by the level controller each time the system called for makeup water. After discussion with the evaporative cooling tower vendor, an engineering change was initiated removing the trip of the spray pump and fan on "1ow" leve1 (raw water makeup) and incorporating a trip on "low-1ow" level in the tower pan. Even at the low-low level, there is adequate water remaining in the system to keep the spray pump suction line flooded. The spray pump and fan will not restart automatically following a low-low level trip; such a condition should be investigated by the operator and the spray pump and fan restarted when the cause of the low-low levet condition is corrected.

Evaporative Cooling Tower Tests: After flushing of the tower pans was completed, each evaporative cooling tower was tested. The fans, spray pumps, 
pan heaters and fan dampers were tested for proper operation. interlocks and controls were verified to operate as designed.

AT1 alarms,

The filtration loop on each system was tested. This testing included the filter pumps, separators, bag filters, conductivity elements and flow switches. Al1 were verified to operate as designed.

Vibration Checks: Vibration data were taken by TWRS maintenance on al1 rotating equipment in each EW cooling system in August 1996. These data are attached as part of a report from a TWRS maintenance consultant, along with recommendations for those components with questionable vibration readings. The items in question included the evaporative cooling tower fans, filter recirc pump AY102-W-P-1, and the condenser cooling recirc pumps for AY102. In addition, the report cited general deficiencies (e.g., loose or missing fasteners, incorrect mounting) in how the components were installed.

Corrective maintenance was initiated in response to the report (as well as the pump seal leakage problem discussed above). Some items have been successfully overhauled and retested, while work is in progress on others. At the time of this writing, all of the condenser recirculation pumps had been overhauled and reinstalled with the exception of AY102-EW-P-1A and AZ102-EW-P-1B.

A final set of readings was taken in October 1997, and the resulting followup report is attached. The fans are not mentioned in the followup; high readings reported earlier were attributed to the motor mounting structures and are inherent in the vendor's skid design, and not indicative of a problem with the motors or fans, per se. The followup report states a "generally acceptable condition" is indicated for all of the pumps, with a few exceptions. One of the condenser recirculation pumps, AZ102-EW-P-1B, remains a serious concern due to vibration and it is recommended that the motor be replaced before turnover to Operations. In addition, the condenser spray pump AZ101-EW-SP-1 is stated to have a bad bearing and will need to be replaced (as a unit, with motor). The two "missing" condenser recirc pumps remain to be reinstalled and retested.

Test Notes: The test procedure required a Test Engineer's signature (but not the date of the signature) on some of the test steps. Dates were provided along with signatures on calibration verification sheets, valve alignment checklists, and electrical alignment checklists (see Appendix-E), as we11 as the "prerequisite" steps in Section 4.0. Performance dates for specific test steps may be verified from archived work packages containing the original test record (see Item 8 of checklist, Attachment 1 ).

Test steps requiring a Test Engineer's signature were those which entailed either recording data, or verifying pretest conditions, system configuration, or expected operating conditions or responses which constitute the acceptance criteria for the test. Recorded data were required primarily for information.

Paragraph 4.7 provides for recording of data for a specific 1 ist of equipment and instruments (M\&TE) required for testing. However, some of the listed items happen not to be required for this test, and no data are entered. Also, in many test steps throughout the procedure, measurement of motor amperage was 
HNF-SD-W030-TD-003, REV. 0, PAGE 7

taken by test instrument rather than read on the graphic screen (the remote current-measuring device was not available at the time); this is considered acceptable since further testing is planned.

This P0TP test effectively satisfies previously-deferred test exception TE\#004 and TE\#005 from construction acceptance testing, reference-7, sections 9.2 and 9.8, "Fluid Cooler Fan Testing". During the ATP test, defects were discovered with the temperature-controlled automatic damper positioning devices for the cooling fans on the $A Z$ fluid coolers. Corrective action was taken Tater and the devices were successfully tested in section 3.0 of Attachment-C and $-D$ of this test.

One test exception was written to remove several items from 1 ists of instrumentation requiring calibration. The instruments in question were functionally tested but were not designed to be caljbrated, therefore the requirement was not relevant.

\section{CONCLUSION}

A11 local and remote controls were verified to operate as designed. All instrumentation and interlocks were functionally checked to operate as designed. No testing was performed to evaluate heat removal capacity as the associated ventilation systems are not operable as yet. Extensive data will be taken as part of the future integrated systems test to aid in this evaluation. The Recirculation Condenser Cooling Systems, AY101, AY102, AZ101 \& AZ102, were found to operate as designed. 


\begin{tabular}{|c|c|c|c|}
\hline \multicolumn{4}{|c|}{ ATTACHMENT 1 - TEST REPORT CHECKLIST } \\
\hline ITEM & REQUIRED ACTION TO BE VERIFIED & INITIALS & DATE \\
\hline 1 & Test completed per approved procedure & 82 & $10 / 15 / 97$ \\
\hline \multirow[t]{8}{*}{2} & Required enclosures provided & & $10 / 15 / 47$ \\
\hline & a. Summary of test results & & $10 / 15 / 47$ \\
\hline & b. Signed/dated procedure validation sheet & & $10 / 15 / 87$ \\
\hline & c. Applicable ECNs and NCRs $N / A$ & te & $10 / 15 / 97$ \\
\hline & d. Onsite vendor test procedures/reports $N / A$ & tc & $10 / 15 / 97$ \\
\hline & e. Applicable/important vendor data & tre & $10 / 15 / 47$ \\
\hline & f. CVI 1ist, Major equipment & tTe & $10 / 15 / 97$ \\
\hline & g. Instrument and Loop calibration data & & $10 / 15 / 47$ \\
\hline \multirow[t]{3}{*}{3} & Test Exceptions (TEs) - Qty. $\quad$ ol & 1C. & $10 / 15 / 97$ \\
\hline & a. All listed in Test Log & & $10 / 15 / 97$ \\
\hline & b. Al1 TE reports closed/signed/dated & & $14 / 15 / 97$ \\
\hline 4 & Al1 applicable test steps signed/dated (1) & & $10 / 15 / 27$ \\
\hline \multirow[t]{3}{*}{5} & Test personnel & xrc & $10 / 15 / 27$ \\
\hline & a. All qualified (2) & E & $10 / 15 / 47$ \\
\hline & b. All have signed/dated signature $10 \mathrm{~g}$ & & $10 / 15 / 97$ \\
\hline \multirow[t]{4}{*}{6} & Recorded test data & gre & $10 / 15 / 47$ \\
\hline & a. Summary of acceptance criteria incl. & $\sqrt{0}$ & $10 / 15 / 97$ \\
\hline & b. AT1 required data entered & $\sqrt{2}$ & $10 / 15 / 97$ \\
\hline & c. All data meets acceptance criteria (3) & & $10 / 15 / 97$ \\
\hline \multirow[t]{3}{*}{7} & Related Acceptance Test Report (ATR) (4) & 积 & $10 / 15 \% 97$ \\
\hline & a. Report No: WHC-SD-WO30-ATR- 004 & & $10 / 15 / 97$ \\
\hline & b. TEs covered in this test: 004,005 (4) & QIE & $20 / 15 / 97$ \\
\hline 8 & Filename, original test record $2 E-96-00285$ & & $10 / 15 / 47$ \\
\hline
\end{tabular}

NOTES: 1. All test steps are signed off by the responsible person. Where the signoff date is not noted, the test summary presents appropriate disposition (Test Notes).

2. No personnel qualification standard was established for this test beyond that required to perform the responsibilities listed in section 2.3 . The individuals assigned were deemed qualified by their management.

3. Where acceptance criteria was not specified in the procedure, it is addressed in the test report.

4. For description of ATR issues, see Test Report (Test Notes). 
HNF-SD-W030-TD-003,

REV. O, PAGE 9

\section{ATTACHMENT 2:}

\section{COPY OF ORIGINAL TEST PROCEDURE WITH RECORDED DATA}


PREOPERATIONAL TESTING, RECIRCULATION CO

WHC-SO-W030-POTP-003

HNF-SD-W030-TD-003,

ION NO. 0

REV. 0 , PAGE 10

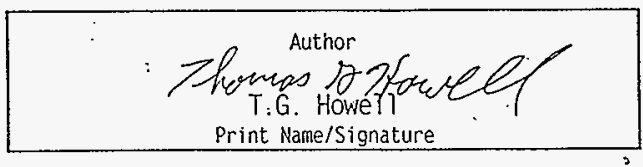

APPROVAL DESIGNATOR SO

PROCEDURE APPROVAL BY TEST: REVIEW BOARD (RB)

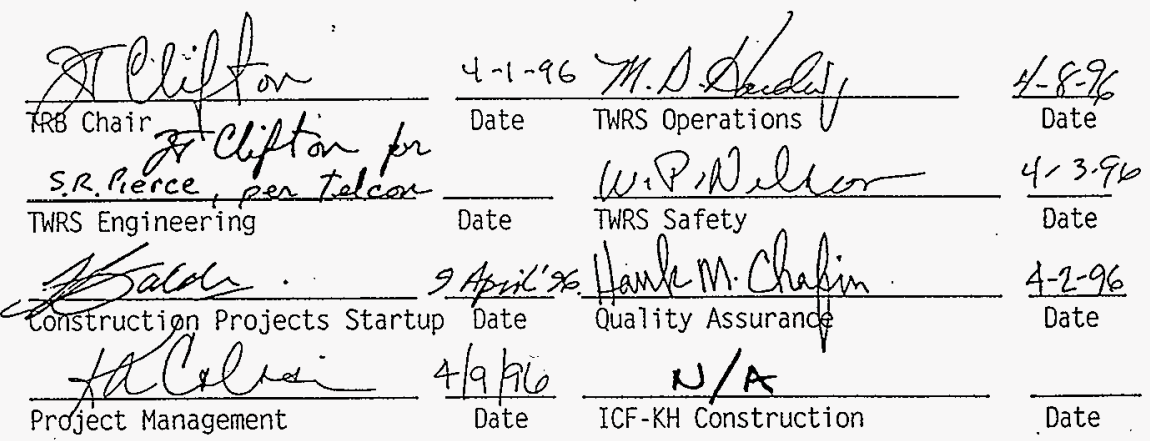




\section{TABLE OF CONTENTS PAGE}

1.0 PURPOSE

2.0 INFORMATION

2.1 SCOPE

2.2 TERMS AND DEFINITIONS

2.3 RESPONSIBILITIES

2.4 CHANGE CONTROL

2.5 EXCEPTIONS

2.6 REFERENCES

2.7 ENVIRONMENTAL

2.8 SAFETY

2:9 RADIATION ANO CONTAMINATION CONTROL

2.11 GENERAL INFORMATION

2.12 LIMITS AND PRECAUTIONS

4.0 PREREQUISITES

5.1 Tank AY101 Recirc Condenser Cooling System

5.2 Tank AY102 Recirc Condenser Cooling System

5.3 Tank AZ101 Recirc Condenser Cooling System

5.4 Tank AZ102 Recirc Condenser Cooling System

Attachment $A$

ar- Attachment B Attachment $\mathrm{C}$ Attachment $D$ 


\subsection{PURPOSE}

1.1 This procedure has been prepared to verify the Recirculation Condenser Cooling Systems operate in accordance with system design.

\subsection{INFORMATION}

\subsection{SCOPE}

2.1.1 This procedure will demonstrate the operation of the following components in the Recirculation Condenser Cooling Systems for Tanks AZ101. AZ102. AY101, and AY102:

- Glycol System Recirc Pumps

- Cooling Tower Spray Pump

- Cooling Fan

- Recirc Pump

- Separator.

- Bag Filter

2.1.2 This test will demonstrate the operation of system interlocks and controls both local and remote.

\subsection{TERMS AND DEFINITIONS}
2.2.1 HPT - Heath Physics Iechnician
2.2.2 OS - Operating Station
2.2.3 LOI - Local Operator Interface device
2.2.4 HS - Hand Switch

\subsection{RESPONSIBILITIES}

2.3.1 The craft (TWRS Maintenance and/or Construction Forces) s.'personnel are responsible for:

- Providing assistance during the test. 
PREOPERATIONAL TESTING, RECIRCULATION CONDENSER COOLING WHC-SD-W03O-POTP-OC

REVISION NO. 0

HNF-SD-HO3O-TD-003, REV. 0, PAGE 13

\subsubsection{Test Director responsible:}

- Safe and productive accomplishment of the tests necessary to achieve startup.

- Ensure safe working conditions and practices.

- Ensure compliance with test documents. Operational

Safety Requirements/Documents (OSRS/OSDs) during testing.

- Communicate and coordinate the tests with the East Tank Farm Shift Manager.

- Ensure appropriate review/approval of any modifications to test.procedures are completed prior to returning to work

- Direct line of communication and centralized point of control during normal. abnorma?, and casualty situations.

- Provides the M\&TE equipment found in STEP 4.7 of this procedure.

- Conducts pre-job planning meeting as necessary.

- Scheduling/rescheduling of the test as required.

- Delegates any of the above responsibilities as needed - to a deputy.

2.3.3 The Engineering Personnel responsible for:

- Providing technical support during testing.

- Providing programming support during testing.

- Forcing data in PLC program during testing.

- Direct preoperational testing

- Review test documents to validate acceptance

- Prepare post testing documents

- Records equipment status and data per this procedure.

- Conducts pre-job system walkdown.

- Recording data exceptions and other notes as required on the POTP Data Sheets.

2.3.4 Operations Personnel responsible for:

- Observing test activities for training purposes. 


\subsection{CHANGE CONTROL}

2.4.1 Test procedure administrative or editorial changes required during testing may be accommodated as exceptions in the released test report. if the changes will not affect operating facility safety, function. or performance and will not compromise or influence test data. Requirement changes, changes to acceptance criteria, or changes to Danger. Caution. Special Precautions, or other safety or environmental instructions in test procedures. prepared as supporting documents must be made by engineering change notice.

\subsection{EXCEPTIONS}

2.5.1 Exceptions to results or to the test procedure will be given a sequential number and recorded on Appendix $H$. Test Exception log sheet. A test exception report. Appendix G. will be filled out to record and disposition each test exception.

\subsection{REFERENCES -}

2.6.1 The following documents were used to write or are referenced in this procedure:

- Project W-030 Startup Test P7an. WHC-SD-W030-SUP-003

- H-2-131067. P\&ID AY101EW Recirc Cond Cooling

- H-2-131068. P\&ID AY102EW Recirc Cond Cooling

- H-2-131069. P\&ID AZ101EW Recirc Cond Cooling

- H-2-131070. P\&ID AZ102EW Recirc Cond Cooling

- Tank Farm Ventilation Upgrade. W-030-C2

- Tank Farm Ventilation Upgrade. W-030-C3

- W-030-P1. Evaporative Cooling Tower

- W-030-P12, Vertical.In-Line Centrifugal Pump

- W-030-P14. Primary/Recirculation Off-Gas Heat Exchangers

-W-030-P18, Gas/Liquid Separator

\subsection{ENVIRONMENTAL}

2.7.1 Spills of hazardous materials (including propylene glycol) should be reported to Environmental Reports group at 3734942 . 
PREOPERATIONAL TESTING, RECIRCULATION CONDENSER COOLING

WHC-SD-W030-POTP-00?

REVISION NO. 0

HNF-SD-W030-TD-003,

REV. 0, PAGE 15

2.7.2 Hazardous and mixed waste should be disposed of according to T0-100-052. or by calling Environmental Waste

Operations at $372-1208$.

\subsection{SAFETY}

Warning - In addition to contámination hazards, operators should be aware of the possibility of coming into contact with poisonous snakes and spiders.

Warning - Exposure to Propylene Glycol can cause gastrointestinal disturbances, nausea, headache and - vomiting and CNS depression. May be harmful by ingestion. inhalation or skin absorption. May cause skin and eye irritation.

2.8.1 The following administrative procedures control work performed in this procedure:

- Safety Manua7. WHC-CM-1-10

- Industrial Hygiene Manual. WHC-CM-1-11.

- Tank Farm Health and Safety Plan (HASP). WHC-SD-WM-HSP-002

\subsection{RADIATION AND CONTAMINATION CONTROL}

2.9.1 None of the work involved in this test will be conducted in a radiation or contamination control area.

\subsection{QUALITY ASSURANCE.}

2.10.1 № Quality Assurance witness or holdpoints are required in this procedure. Quality Assurance shall review and approve the test procedure. the final test report and the disposition of all test exceptions.

\subsection{GENERAL INFORMATION}

\subsubsection{None}


PREOPERATIONAL TESTING. RECIRCULATION CONDENSER COOLING

WHC - SD-W030 - POTP - 00'

REVISION NO. 0

HNF-SD-W030-TD-003,

REV. 0, PAGE 16

\subsection{LIMITS AND PRECAUTIONS}

2.12.1 If during performance of this procedure, any of the following conditions are found. immediately notify the Test Director:

- Any equipment malfunction which could prevent fulfillment of it's functional requirements.

- Personnel error or procedural inadequacy which could prevent fulfillment of procedural requirements.

The Test Director may choose to stop work and place equipment in a safe condition based on the significance of the malfunction. error or inadequacy.

2.12.2 Contact Test Director for additional instructions if changing plant conditions affect work or delays in work extend past end of shift.

2.12.3 If any waste is generated during performance of this. instruction consult Facility/Plant/Area Hazardous Waste Coordinator for specific instructions to ensure compliance with WHC and DOE environmental standards, as applicable. for disposal.

2.12.4 Comply with WHC and plant/facility specific lock and tag or over-tagging requirements, as applicable.

2.12.5 A11 Measuring and Test Equipment (M\&TE) used during performance of this procedure to collect qualitative data. with the exception of timing devices shall meet the following requirements:

- Be within its current calibration cycle as evidenced by an affixed calibration label.

- Be capable of desired range.

- Have an accuracy (consistent with state-of-the-art limitations) equal to or greater than the accuracy specified in the procedure.

2.12.6 Timing measurements shall be made with commercially available time devices. 
2.12.7. The Test Director has overall control of the testing process and change authorization for this procedure. They are responsible for running the test, data collection. and ensuring compliance with all requirements in this procedure.

2.12.8 All readings are to be taken and recorded for each location where the capability exists (i.e. local instrument. LOI, OS).

\subsection{RECORDS}

3.1 This procedure as well as all completed attachments/appendices are kept as a permanent record.

\subsection{PREREQUISITES}

Unlèss otherwise specified, prerequisite actions may be performed in any order.

4.1 Perform a pretest briefing for all personnel involved in the performance of this test.

Test Director

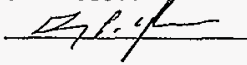
Date $s=-14-86$

4.2 Perform a walkdown inspection of the systems tested by this procedure.

Test Director

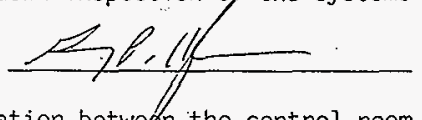
Date $5-14-86$

4.3 Radio communication between the control room and field test personnel has been verified

Test Director
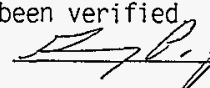
Date $5-14-86$

4.4 The official copy of this POTP and all other copies that will be

ar. used during the test have been verffied to be the latest revision. Test Director

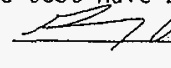
Date $=M 4 y$

4.5 All open items have been evaluated and verified to not affect the performance of this POTP (Quality Assurance Nonconformance Reports. Construction Punch Lists, outstanding Engineering or Field Change Notices. Startup-originated Design Change Requests. Test Deficiency Reports, and Master System Purch List items).

Test Director

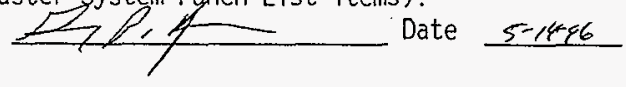


4.6 Notify WHC Maintenance (373-9035) 24 hours prior to commencement of

- testing to facilitate obtaining baseline data on fans and motors by maintenance.

\subsection{EQUIPMENT/INSTRUMENTS}

Supplied by Test Operator uniess otherwise noted.

4.7.1 Clamp-on Ammeter: 0-40 Ampere.

Manufacturer: Amp pesclie Model No. $A C \Omega-4$

Serial No. Olaq Calibration Date 1-24-96

Calibration Due Date 1-24,97

4.7.2 Shorting Jumpers with ON-OFF switch (SW): 4 feet long.

Quantity of 2 required.

4.7.3 Sonic Flow Instrument with a range of $0-150 \mathrm{gpm}$ with a minimum line size of $1 \frac{1}{2}$ inch I.D.

Manufacturer: - L.PA Model No. AL/A Serial No. N/A Calibration Date Calibration Due Date N N/A

4.7.4 Multi-meter 0 -480V

Manufacturer: Eluke Model No. $8024 B$

Serial No. O/68 Calibration Date $5-17-95$ Calibration Due Date $5=17-96$

4.7.5 Ohmmeter (OHM): Quant ity of 2 required.

Manufacturer:

Serial No. Model No

Calibration Due Date

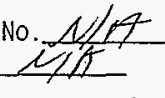

\subsection{PROCEDURE}

6.1 Tank AY101 Recirc Condenser Cooling System.

5.1.1 Preoperational testing shall be performed using Attachment A of this procedure.

5.2 Tank AY102 Recirc Condenser Cooling System.

5.2.1 Preoperational testing shall be performed using Attachment $B$ of this procedure. 
HNF-SD-W030-TD-003, REV. 0, PAGE 19

5.3 Tank AZ101 Recirc Condenser Cooling System.

5.3:1 Preoperational testing shall be performed using Attachment $C$ of this procedure.

5.4 Tank AZ102 Recirc Condenser Cooling System.

5.4.1 Preoperational testing shall be performed using Attachment D of this procedure. 


\begin{tabular}{ccc}
\hline PREOPERATIONAL TESTING. RECIRCULATION CONDENSER COOLING \\
WHC-SD-W030-POTP-003 & HNF-SD-WO30-TD-003, \\
ATTACHMENT A & REV. 0, PAGE 20 \\
Revision No. $0 \quad$ TANK AY101 RECIRC CONDENSER COC &
\end{tabular}

1.0 INITIAL CONDITIONS

1.1 VERIFY all system instrumentation in Appendix $A$ is calibrated and has a current calibration tag affixed to each instrument and that all loop calibrations are complete.

Test Engineer:

1.2 VERIFY the system is aligned for preoperational testing in

accordance with Appendix $B$.

Test Engineer: $>$ Pff

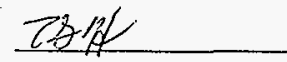

1.3 VERIFY system electrical circuit breakers are aligned in accordance with Appendix $C$.

Test Engineer:

JAht

1.4 VERIFY expansion tank level is at mid level as indicated on LIAY101EWTK-1.

Test Engineer: TAHF

1.5 All personinel who will be involved with this procedure have provided the required signature verification information in Appendix E .

Test Engineer:

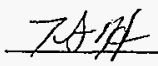

1.6 The test engineer has verified, by review of the tag $\log$ and a walkdown of the systems being tested, that all components within and including the test boundary have been "blue" tagged.

Test Engineer:

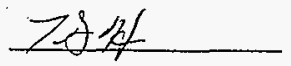

2.0 Tank AY101 Glycol Recirc System Testing

2.1 VERIFY Recirc Pump AY101-EW-P-1A-10cal control switch HS-AY101EWPIAI in the STOP pOsition.

Test Engineer:

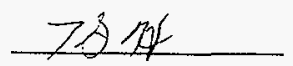

2.2 CLOSE local breaker for Recirc Pump AY101-EW-P.-1A. 
PREOPERATIONAL TESTING, RECIRCULATION CONNFNSFR INOI TNR WHC-SD-W030-POTP-003

Revision No. $\underline{0}$

2.3 RECORD the following data for Recirc Pump AY101-EW-P-1A:

Expansion Tank Level (Local)

Suction Pressure(Loca1) Run Time (graphics screen 06EvpAYI.v) $\bigcirc$ hrs

Test Engineer:

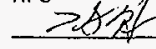

2.4 ATTEMPT to start Recirc Pump AY101-EW-P-1A from MCS graphics screen 06EvpAY1.v.

2.5 VERIFY Recirc Pump AY101-EW-P-IA does not start.

Test Engineer:

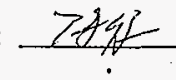

2.6 BUMP Recirc Pump AY101-EW-P-1A by placing control switch HSAY101EWP-IAI in START and then back to STOP.

2.7 VERIFY rotation of the Recirc Pump AY101-EW-P-1A is in the direction indicated by the arrow on the pump.

Test Engineer:

2.8 PLACE Recirc Pump AY101-EW-P-1A 10cal control switch HS-AY101EWP$1 A 1$ in the START position.

2.9 VERIFY Recirc Pump AY101-EW-P-1A starts.

Test Engineer:

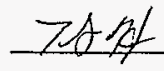

2.10 RECORD the following data for Recirc Pump AY101-EW-P-1A:

2.10.1

TesT Jestrumput

JOHA: $5 \$ 4196$
2.10.2 Amps
Motor voltage (TEST INST.) 492 volts
Suction Pressure (LOCAL)
- Discharge Pressure (LOCAL)

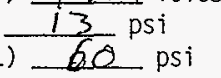

2.10 .3
2.10 .4

2.10 .3
2.10 .4

Motor Current (

Test Engineer:

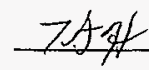

2.11 SLOWLY OPEN Recirc pump AY101-EW-P-1A discharge valve HV-AY101EWP$1 A 5$ and establish a system flowrate of $\geq 192 \mathrm{gpm}$ as indicated on FI-AY101EWR-1 on graphics screen 06EvpAY1.v. 
PREOPERATIONAL TESTING, RECIRCULATION CONDENSER COOLING WHC-SD-W030-POTP-O

Revision No. 0

ATTACHMENT A

HNF-SD-WO30-TD-003,

REV. 0, PAGE 22

TANK AY101 RECIRC CONDENSER

2.12 VERIFY low flow alarm FAL-AY101WR-I clears graphics screen 06EvPAY1.V.

Test Engineer: ZA $\not 2$

2.13 RECORD the following data for Recirc Pump AY101-EW-P-1A:

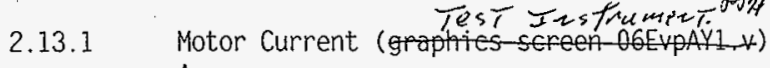
Amps

2.13 .2

2.13 .3

2.13 .4

2.13 .5

2.13 .6

Motor voltage (TEST INST.) 494 volts Suction Pressure (LOCAL) H.s psi

Discharge Pressure (LOCAL) -58 psi

System Flow (graphics screen 06EvpAY1.v) 192 gpm

Expansion Tank Level (Local) $1 / 2$

Test Engineer:

2.14 THROTTLE CLOSED valve HV-AY101EWS-1A2 untiT system flow starts to decrease as indicated on FI-AY101EWR-1 graphics screen .06EvpAY1.v.

2.15 FULLY OPEN Recirc PUMP AY101-EW-P-IA discharge valve HV-AY101EWPIA5.

2.16 ADJUST system flow to $2192 \mathrm{gpm}$ using HV-AY101EWS-1A2

2.17 VERIFY Recirc Pump AY101-EW-P-1B local control switch HS-AY101EWP$1 B 1$ in the STOP position.

Test Engineer:

2.18 CLOSE local breaker for Recirc Pump AY101-EW-P-1B.

2.19 RECORO the following data for Recirc Pump AY101-EW-P-1B:

Suction Pressure(Local) 11.5 psi

Run Time (graphics screen 06EvpAY1.v) \& hrs.

-. Test Engineer:

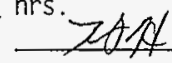

2.20 BUMP Recirc Pump AY101-EW-P-1B by placing control switch HSAY101EWP-1B1 in START and then back to STOP.

2.21 VERIFY rotation of the Recirc Pump AY101-EW-P-1B is in the direction indicated by the arrow on the pump.

Test Engineer:

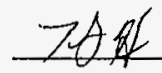


PREOPERATIONAL TESTING, RECIRCULATION RONDENSER COOLING WHC-SD-W030-POIP.0O

Revision No. 0 ATTACHMENT A HNF-SD-W030-TD-003, TANK AY101 RECIRC CONDENSER

2.22 ATTEMPT to start Recirc Pump AY101-EW-P-1B from MCS graphics. screen 06EvpAY1.v.

2.23 VERIFY Recirc Pump AY101-EW-P-1B does not start.

Test Engineer:

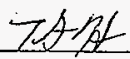

2.24 PLACE Recirc Pump AY101-EW-P-IB local control switch HS-AY101EWP$1 B 1$ in the START position.

2.25 VERIFY Recirc Pump AY101-EW-P-IB starts.

Test Engineer:

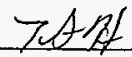

2.26 RECORD the following data for Recirc Pump AY101-EW-P-1B: TesT Justrumery Tons shislog

2.26.1 Motor Current (graphies-sereen-06EupAY1.*) 7.0

2.26.2 Motor voltage (TEST INST.) 494 volts

2.26.3 Suction Pressure (LOCAL) $/ 3 \mathrm{psi}$

2.26.4 Discharge Pressure (LOCAL) $\frac{52}{2} \mathrm{psi}$

Test Engineer: 건

2.27 SLOWLY OPEN Recirc pump AY101-EW-P-1B discharge vaive HV-AY101EWP185.

2.28 ATTEMPT to stop Recirc Pump AY101-EW-P-1A from MCS graphics screen 06EvpAYl.v.

2.29 VERIFY that Recirc Pump AY101-EW-P-IA does not STOP.

Test Engineer:

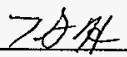

2.30 PLACE Recirc Pump AY101-EW-P-iA local control switch HS-AY101EWP-

\%. IAI in the STOP position.

2.31 VERIFY that Recirc Pump AY101-EW-P-1A STOPS.

Test Engineer:

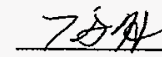

2.32 ADJUST system flow, if required, to $\geq 192$ gpm using HV-AY101EWS$1 \mathrm{~A} 2$. 
PREOPERATIONAL TESTING, RECIRCULATION CONNFNCFR IONI INS WHC-SD-W030-POTP-003

Revision No. 0 ATTACHMENT A

HNF-SD-W030-TD-003, TANK AY101 RECIRC CONDENSER CI

2.33 RECORD the following data for Recirc Pump AY101-EW-P-1B:

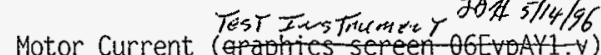

Motor Current (graphies-sereen-06EvAY1-v) 9,4 Amps

2.33.2 Motor Voltage (TEST INST.) 484 Volts

2.33.3 Suction Pressure (LOCAL) M.5 psi

2.33.4 Discharge Pressure (LOCAL) $4 \pi$ psi

2.33.5 System Flow (graphics screen 06EvpAYl.v) $19 \geq \mathrm{gpm}$

2.33.6 Expansion Tank Level (Local) _ $y_{2}$

Test Engineer:

2.34 THROTTLE HV-AY101EWS-1A2 to decrease flow to $<170 \mathrm{gpm}$.

2.35 VERIFY system low flow alarm. FAL-AY101EWR-1 annunciates on graphics screen 06EvpAY1.v.

Test Engineer:

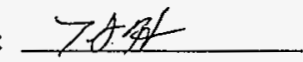

2.36 THROTTLE HV-AY101EWS-1A2 to increase flow to $2192 \mathrm{gpm}$.

2.37 VERIFY system low flow alarm. FAL-AY101EWR-1 clears on graphics screen 06EvpAY1.v.

Test Engineer:

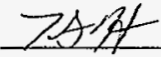

2.38 PLACE Recirc Pump AY101-EW-P-1A local control switch HS-AY101EWP$1 A 1$ in the REMOTE position.

2.39 START Recirc Pump AY101-EW-P-1A from graphics screen 06EvpAY1.v

2.40 VERIFY Recirc Pump AY101-EW-P-1A starts.

Test Engineer:

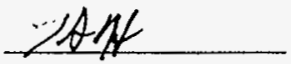

2.A1 PLACE Recirc Pump AY101-EW-P-1B local contro? switch HS-AYI01EWP$1 B 1$ in the STOP position.

2.42 VERIFY Recirc Pump AY101-EW-P-1B stops.

Test Engineer:

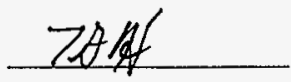

2.43 PLACE Recirc Pump AY101-EW-P-1B local control switch HS-AY101EWP$1 B 1$ in the REMOTE position.

2.44 PLACE Recirc Pump AY101-EW-P-1B in Standby on graphics screen 06EVPAY1.V. 


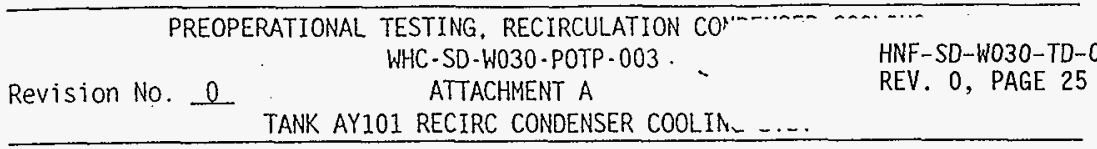

2.45 OPEN local breaker switch for Recirc Pump AY101-EW-P-1A.

2.46 VERIFY Recirc Pump AY101-EW-P-1B auto starts. Test Engineer:

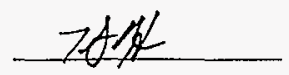

2.47 PLACE Recirc Pump AY101-EW-P-IA remote control switch HS-AY101EWP$1 A$ in STOP position on graphics screen 06EvpAYl.V.

2.48 CLOSE local breaker for Recirc Pump AY101-EW-P-1A.

2.49 PLACE Recirc Pump AY101-EW-P-1A in the Standby on graphics screen 06EvPAY1.V.

2.50 OPEN local breaker for Recirc Pump AY101-EW-P-1B.

2.51 VERIFY Recirc Pump AY101-EW-P-1A auto starts. Test Engineer:

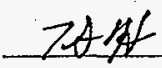

2.52 PLACE Recirc. Pump AY101-EW-P-1B remote control switch HS-AY101EWP$1 B$ in STOP position on graphics screen 06EvPAYl.V.

2.53 CLOSE loca'l breaker for Recirc Pump AY101-EW-P-1B.

2.54 ALLOW Recirc Pump AY101-EW-P-1A to operate for one hour for collection of vibration data and bearing data.

2.55 RECORD vibration data and bearing temperatures on Appendix D-1.

Test Engineer:

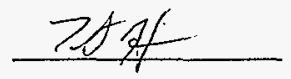

2.56 START Recirc Pump AY101-EW-P-1B remotely from LCU4-17.

2r:57 STOP Recirc Pump AY101-EW-P-1A remotely from LCU4-16.

2.58 ALLOW Recirc.Pump AY101-EW-P-1B.to operate for one hour for collection of vibration data and bearing data.

2.59 RECORD vibration data and bearing temperatures on Appendix D-2.

Test Engineer:

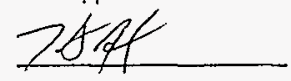


PREOPERATIONAL TESTING, RECIRCULATION I WHC-SD-W030-POTP-003
HNF-SD-W030-TD-003, REV. 0, PAGE 26

Revision No. 0

ATTACHMENT A

TANK AY101 RECIRC CONDENSER COOLAMG JIMIII

2.60 SLOWLY CLOSE Expansion Tank Level Switch LSL-AY101EWTK- 1 Low Side Isolation Valve HV-AY101EWTK-1Al.

2.61 VERIFY Expansion Tank Low Level Alarm LAL-AY101EWTK-1 annunciates on graphics screen 06EvpAY1.v.

Test Engineer:

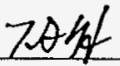

2.62 OPEN Expansion Tank Level Switch LSL-AY101EWTK- 1 Low Side Isolation Valve HV-AY101EWTK-1A1.

2.63 VERIFY Expansion Tank Low Level ATarm LAL-AYI01EWTK-1 clears on graphics screen 06EvpAY1.v.

Test Engineer:

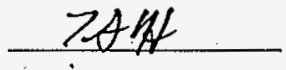

2.64 PLACE LOW Flow Shutdown switches HS-AY101EWP-IAB and HS-AY101EWP$1 B B$ in the OVERRIDE position on graphics screen 06EvpAYl.v.

2.65 PLACE Recirc Pump AY101-EW-P-1A in STANDBY on graphics screen 06EvPAY1.V.

2.66 THROTTLE HV-AY101ENS-1A2 to decrease flow to <100 gpm.

2.67 VERIFY the Recirc Pump AY101-EW-P-1B does not STOP on low flow.

Test Engineer:

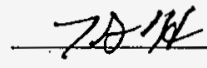

2.68 PLACE Low Flow Shutdown Override switch HS-AY101EWP-1BB in the: NORMAL position from the LOI.

2.69 VERIFY the Recirc Pump AY101-EW-P-1B STOPS on low flow.

Test Engineer:

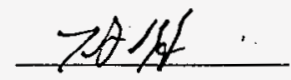

2.70 VERIFY Recirc Pump AY101-EW-P-1A starts.

Test Engineer:

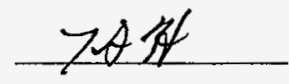

2.71 PLACE Low Flow Shutdown Override switch HS-AY101EWP-IAB in the NORMAL position on graphics screen 06EvpAY1.V.

2.72 VERIFY the Recirc PUMP AY101-EW-P-1A STOPS on low flow.

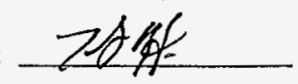


PREOPERATIONAL TESTING, RECIRCULATION CONDFNSER COOLING WHC - SO - W030-P0TP- 003

Revision No. 0

ATTACHMENT A

HNF-SD-W030-TD-003,

REV. 0, PAGE 27

TANK AY101 RECIRC CONDENSER CC

2.73 PLACE Low Flow Shutdown Override switch HS-AY101EWP-1AB in the OVERRIDE position on graphics screen 06EvpAY1.V.

2.74 RESET Recirc Pump AY101-EW-P-1A by placing remote control switch HS-AY101EWP-1A in STOP position on graphics screen 06EvPAYI.v.

2.75 PLACE Recirc Pump AY101-EW-P-1A remote control switch HS-AY101EWP$1 A$ in START position on graphics screen 06EvpAY1.V.

2.76 VERIFY that Recirc Pump AY101-EW-P-1A starts.

Test Engineer:

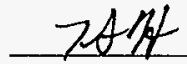

2.77 THROTTLE HV-AY101EWS-1A2 to increase system flow to $2192 \mathrm{gpm}$.

2.78 PLACE LOW Flow Shutdown Override switch HS-AY101EWP-1AB in the NORMAL position on graphics screen 06EvpAY1.v.

3.0 Tank AY101 Evaporator Tower Testing

3.1 REMOVE manway covers on the cooling tower.

3.2 OPEN HV-AY101-RW-1 Cooling Tower Raw Water Isolation vaive at Evaporator Tower.

3.3 VERIFY cooling tower fill via makeup valve EV-AY101EWT-1AI.

\section{Test Engineer: ZA HL}

3.4 INSTALL manway covers when cooling tower fill is complete.

3.5 VERIFY the following local switch positions:

- Evaporator Tower AY101-EW-T-1 Spray Pump AY101-EW-SP-1 loca1 control switch HS-AY101-EWSP-1A in OFF.

- Evaporator Tower AY101-EW-T-1 Fan 10cal control switch HSAY101EWT-1A1 in OFF.

- Evaporator Tower AY101-EW-T-1 Pan Heater local control switch HS-AY101EWT-1A2 in OFF.

Test Engineer:

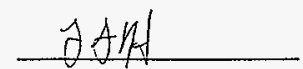

3.6 CLOSE the local supply breaker for the spray pump. cooling fan. and pan heater in the Evaporator Tower local distribution panel. 
PREOPERATIONAL TESTING, RECIRCULATION CONDENSER COOLING WHC-SD-W030-POTP- 003

Revision No. 0 ATTACHMENT A

HNF-SD-W030-TD-003, TANK AYIO1 RECIRC CONDENSER CC REV. 0, PAGE 28

3.7 REMOVE splash guard from top of Spray Pump AY101-EW-SP-1 motor to enable viewing of the motor shaft.

3.8 BUMP Spray Pump AY101-EW-SP-1 by placing control switch HS-AY101EWSP-1A in START and then back to OFF.

3.9 VERIFY rotation of the Spray Pump AY101-EW-SP-1 is in the direction indicated by the arrow on the pump.

Test Engineer:

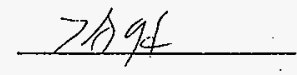

3.10 REINSTALL splash guard on Spray Pump AY101-EW-SP-1 motor.

3.11 PLACE Evaporator Tower AY101-EW-T-1 Spray Pump AY101-EW-SP-1 local control switch HS-AY101-EWSP-1A in START:

3.12 VERIFY Spray Pump AY101-EW-SP-1 starts.

Test Engineer:

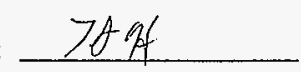

3.13 RECORD the following data for Spray Pump AY101-EW-SP-1:

3.13.1 Motor Current (TEST INST.) 1.5 Amps

3.13.2 Motor Voltage (TEST INST.) 482 Volts

3.13.3 Spray Flow (TEST INST.) $\overline{1 / T \text { TP }}$ gpm

Test Engineer:

3.14 ALLOW Spray Pump AY101-EW-SP-1 to operate for one hour for collection of vibration data and bearing data.

3.15 RECORD vibration data and bearing temperatures on Appendix D-3.

Test Engineer:

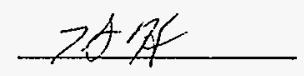

30.16 PLACE Evaporator Tower AY101-EW-T-1 Spray Pump AY101-EW-SP-1 remote control switch HS-AY101-EWSP-1 in STOP on graphics screen 06EvPAY1.v.

3.17 VERIFY Spray Pump AY101-EW-SP-1 does NOT STOP. Test Engineer:

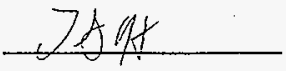

3.18 PLACE Evaporator Tower AY101-EW-T-1 Temperature Control Damper local control Switch HS-AY101-EWT-1A3 in OPEN. 
PREOPERATIONAL TESTING, RECIRCULATION RONANNCED IONI TMR: WHC-SD-W03O-POTP-003

HNF-SD-W030-TD-003,

Revision No. $\underline{0}$

ATTACHMENT A

REV. O, PAGE 29

TANK AY101 RECIRC CONDENSER CC

3.19 VERIFY Evaporator Tower AY101-EW-T-1 Temperature Control Damper OPENS.

Test Engineer:

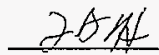

3.20 PLACE Evaporator Tower AY101-EW-T-1 Cooling Fan local control. switch HS-AYI01-EWT-1A1 in START.

3.21 VERIFY Evaporator Tower AY101-EW-T-1 Cooing Fan starts.

Test Engineer:

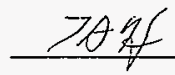

3.22 RECORD the following data for Evaporator Tower AY101-EW-T-1 Cooling Fan:

3.22.1 Motor Current (TEST INST.) 10.4 AmpS

3.22.2 Motor voltage (TEST INST.) $4 \$ 2$ Volts

Test Engineer:

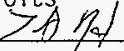

3.23 ALLOW Evaporator Tower AY101-EW-T-1 Cooling Fan to operate for one hour for collection of vibration data and bearing data.

3.24 RECORD yibration data and bearing temperatures on Appendix D-4.

Test Engineer:

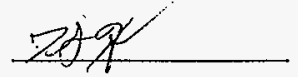

3.25 PLACE Evaporator Tower AY101-EW-T-1 Cooling Fan remote control switch HS-AY101-EWT-1 in OFF on graphics screen 06EvpAY1.v.

3.26 VERIFY Evaporator Tower AY101-EW-T-1 Cooling Fan does NOT STOP..

Test Engineer:

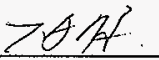

3:27 PLACE Evaporator Tower AY101-EW-T-1 Pan Heater local control switch HS-AY101-EWT-1A2 in ON.

3.28 VERIFY Evaporator Tower AY101-EW-T-1 Pan Heater remains deenergized by checking the heater contactor is OPEN.

Test Engineer:

3.29 SIMULATE a low Evaporator Tower Pan level.

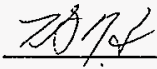


PREOPERATIONAL TESTING, RECIRCULATION CONDENSER COOI ING WHC-SD-W03O-POTP-00

Revision No. 0 ATTACHMENT A HNF-SD-W030-TD-003, REV. O, PAGE 30 TANK AY101 RECIRC CONDENSER

3.30 VERIFY the following actions occur:

3.30.1 Evaporator Tower Spray Pump AY101-EW-SP-1 stops.

3.30.2 Evaporator Tower Cooling Fan stops.

3.30.3 Evaporator Tower Makeup Vaive EV-AY101EWT-1A1 opens. Test Engineer:

3.31 RECORD makeup flow at FI-AZRW-1 (graphics screen 05Water.v). 21 gpm.

Test Engineer:

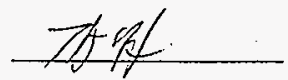

3.32 RESTORE the Evaporator Tower Pan level to normal.

3.33 VERIFY Evaporator Tower Pan Heater energizes by checking the heater contactor is CLOSED.

Test Engineer:

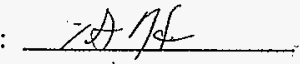

3.34 PLACE Evaporator Tower AY101-EW-T-1 Temperature Contro? Damper local control Switch HS-AY101-EWT-1A3 in AUTO.

3.35 PLACE Evaporator Tower AY101-EW-T-1 Spray Pump AY101-EW-SP-1 locaT control switch HS-AY101-EWSP-1A in REMOTE.

3.36 PLACE Evaporator Tower AY101-EW-T-1 Cooling Fan local control switch HS-AYIO1-EWT-IAI in REMOTE.

3.37 PLACE Evaporator Tower AY101-EW-T-1 Spray Pump AY101-EW-SP-1 remote control switch HS-AY101-EWSP-1 in START on graphics screen 06EvpAY1.v.

3.38 VERIFY Evaporator Tower AY101-EW-T-1 Spray Pump AY101-EW-SP-1 starts.

Test Engineer:

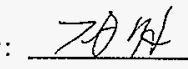

3.39 VERIFY Evaporator Tower Pan Heater deenergizes by checking the heater contactor is OPEN.

Test Engineer: ZA

3.40 PLACE Evaporator Tower AY101-EW-T-1 remote control switch HSAY101-EWT-1A1 in ON on graphics screen 06EvpAY1.v. 
PREOPERATIONAL TESTING, RECIRCULATION CONDENSFR C.OMI TNR.

WHC.SD-W030-POTP.003

Revision No. 0

ATTACHMENT A

HNF-SD-W030-TD-003,

REV. 0, PAGE 31

TANK AY101 RECIRC CONDENSER COOI

3.41 VERIFY the following events occur:

3.41.1 AY101 Evaporator Tower Copling Fan starts.

3.41.2 AY101 Evaporator Tower-AY101-EW-T-1 Temperature

Control Damper modulates to maintain glycol

temperature at setpoint $100^{\circ} \mathrm{F}$ as indicated on graphics screen06EvpAY1.v.

Test Engineer:

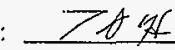

3.42 OPEN Closed Loop Filtration System inlet and outlet block valves HV-AY101W-1 and HV-AY101W-3.

3.43 BUMP the Closed Loop Filtration System Recirc Pump AY101-W-P-1 by placing control switch HS-AY101WP-1 in HAND and then back to OFF.

3.44 VERIFY rotation of the Closed Loop Filtration System Recirc Pump AY101-W-P-1 is in the direction indicated by the arrow on the pump.

Test Engineer:

3.45 PLACE the Closed Loop Filtration System Recirc Pump AY101-W-P-I local control switch AY101WP-1 in HAND.

3.46 VERIFY Closed Loop Filtration Recirc Pump AY101-W-P-1 starts.

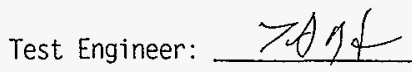

3.47 SLOWLY OPEN Closed Loop Filtration System Recirc Pump AY101-W-P-1 discharge valve HV-AYlOLW-2 to establish full system flow.

3.48 RECORD the following data for Closed Loop Filtration Recirc Pump AY101-W-P-1:

2. 3.48 .1 Motor Current (TEST INST.) $Z .7$ Amps

3.48.2 Motor Voltage (TEST INST.) $48 \tilde{5}$ Volts

3.48.3 Recirc Flow (TEST INST.) MCNAgpm

3.48.4 Separator Inlet Pressure (LOCAL) 23.5 psi

3.48.5 Separator Out let Pressure (LOCAL)

Test Engineer:

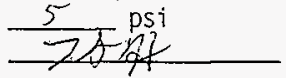

3.49 ALLOW Closed Loop Filtration Recirc Pump AY101-W-P-1 to operate for one hour for collection of vibration data and bearing data. 
PREOPERATIONAL TESTING, RECIRCULATION CONDENSER COOLING WHC-SD-W030-POTP-003

Revision No. 0 ATTACHMENT A

HNF-SD-W030-TD-003, TANK AY101 RECIRC CONDENSER C

REV. 0, PAGE 32

3.50 RECORD vibration data and bearing temperatures on Appendix D-5.

Test Engineer:

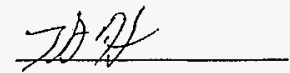

3.51 OPEN Bag Filter AY101-W-F-2 inlet isolation vaive HV-AY101W-4.

3.52 VENT all air from the filter housing using the pressure/vacuum relief valve on top of the filter.

3.53 OPEN Bag Filter AY101-W-F-2 outlet isolation valve HV-AY101W-5.

3.54 VERIFY bag filter differential pressure indicates in the green range on PDIS-AY101WF-2.

Test Engineer:

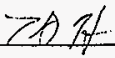

3.55 LOOSEN fitting on Bag Filter PDIS-AY101WF-2 low side instrument tubing.

3.56 VERIFY Bag Filter high differential pressure alarm PDAH-AY101WF-2 initiates at 15psid on graphics screen 06EvpAYl.v.

Test Engineer:

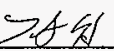

3.57 TIGHTEN fitting Bag Filter PDIS-AY101WF-2 low side instrument line.

3.58 VERIFY Bag Filter high differential pressure alarm PDAH-AY101WF-2 clears on graphics screen 06EvpAYl.v.

Test Engineer:

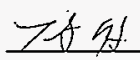

3.59 RECORD the conductivity reading indicated on the Clearview controller.

3.59 .1 Tower fluid conductivity $\frac{240}{\text { Test Engineer: }}$ Micromhos

3.60 PLACE the Test Switch on the Clearview Controller in the TEST position.

3.61 VERIFY Evaporator Tower Bleed Valve EV-AY101EWT-1A2 OPENS.

Test Engineer:

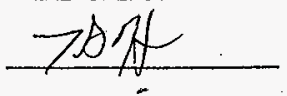


PREOPERATIONAL TESTING, RECIRCULATION CONDENSER COOLING WHC-SD - W030-POTP-00:

Revision No. O ATTACHMENT A

HNF-SD-W030-TD-003, TANK AY101 RECIRC CONDENSER ( REV. 0, PAGE 33

3.62 PLACE the Test Switch on the Clearview Controller in the OPER position.

3.63 VERIFY Evaporator Tower Bleed Valve EV-AY101EWT-1A2 CLOSES.

Test Engineer:

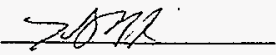

3.64 PLACE the Closed Loop Filtration System Recirc Pump AY101-W-P-1 local control switch AY101WP-1 in OFF.

3.65 CLOSE the Closed Loop Filtration System inlet and outlet block valves HV-AY101W-1 and HV-AY101W-3.

3.66 IF desired. THEN DRAIN the Closed Loop Filtration System as follows, OTHERWISE continue at step 3.67:

3.66.1 REMOVE drain plug from Closed Loop Filtration System

Recirc Pump casing.

3.66.2 DISCONNECT hoses on either side of bag filter.

3.66.3 REMOVE vent plug from top of vent filter.

3.66.4 WHEN system completes draining. THEN reinstall a11 plugs and connect hoses to bag filter.

3.67 PLACE Evaporator Tower AY101-EW-T-1 Spray Pump AY101-EW-SP-1 remote control switch HS-AY101-EWSP-1 in STOP.

3.68 VERIFY Evaporator Tower AY101-EW-T-1 Spray Pump AY101-EW-SP-1 STOPS.

Test Engineer:

3.69 PLACE Evaporator Tower AY101-EW-T-1 remote control switch HSAY101-EWT-1AI in OFF.

3.70. VERIFY the following events occur:

3.70.1 AY101 Evaporator Tower Cooling Fan STOPS.

3.70.2 AY101 Evaporator Tower AY101-EW-T-1 Temperature Control Damper OPENS.

Test Engineer:

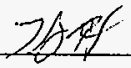

3.71 CLOSE Raw Water supply to the Evaporator Tower HV-AY101RW-1. 


PREOPERATIONAL TESTING, RECIRCULATION CONDENSER COOLING
WHC-SD-WO30.POTP-Or
ATTACHMENT A

3.72 IF it is desired to drain the Evaporator Tower. THEN PERFORM the following steps. OTHERWISE continue at step 3.75.

3.73 PLACE the Test Switch on the Clearview Controller in the TEST position to allow the Evaporator Tower to drain.

3.74 PLACE the Test Switch on the Clearview Controller in the OPER position.

3.75 VERIFY a 7 test equipment installed to support this test has been removed.

Test Engineer: Pof 


\begin{tabular}{c}
\hline PREOPERATIONAL TESTING, RECIRCULATION CONNFNSFR CONI ING \\
WHC.SD-WO30-POTP.003 \\
ATTACHMENT A
\end{tabular}

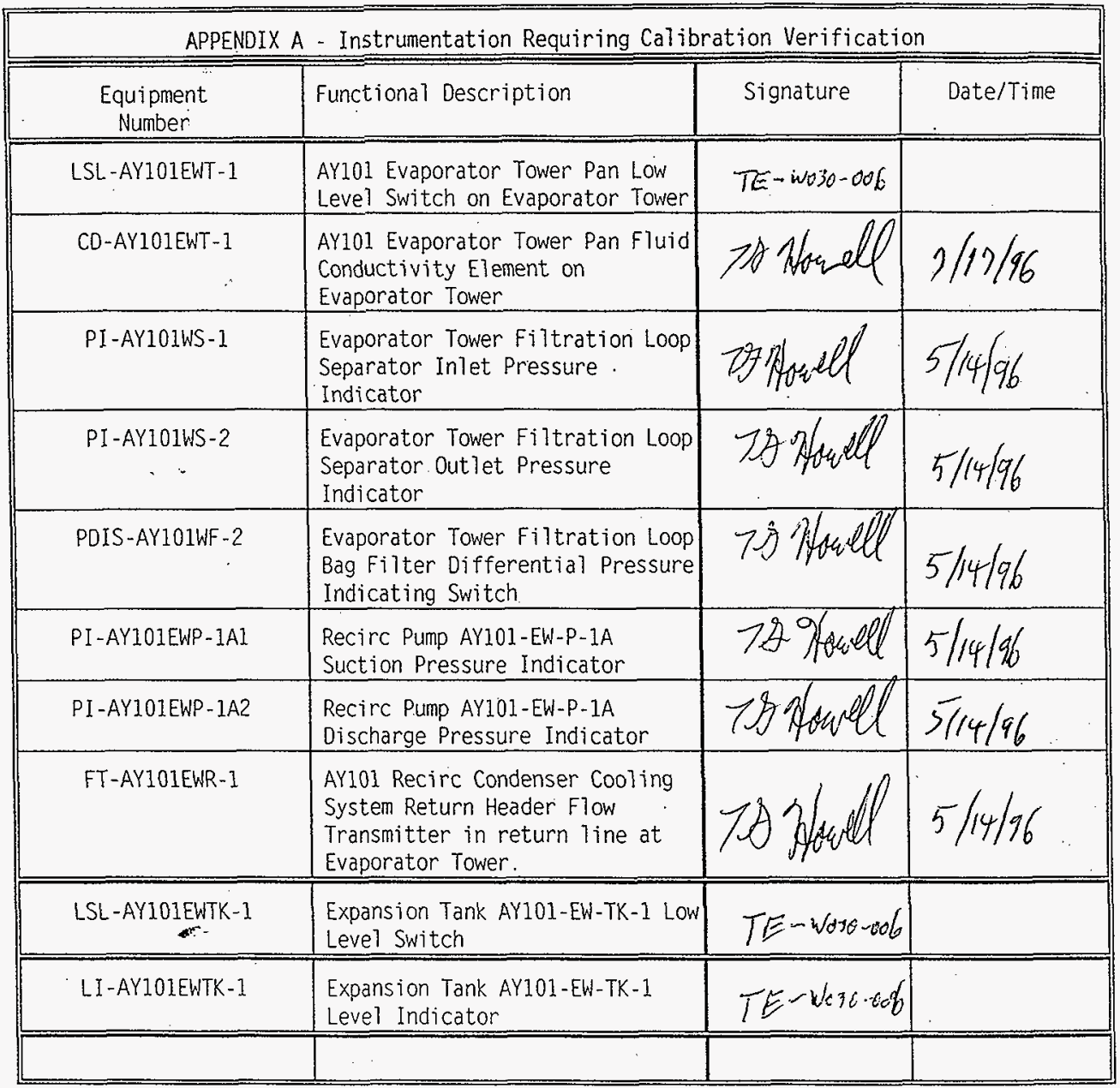


PREOPERATIONAL TESTING. RECIRCULATION CONDENSER C

HNF-SD-W030-TD-003, WHC-SD-W030-POTP-003

Revision No. 0

ATTACHMENT A

Effective Date

TANK AY101 RECIRC CONDENSER COOLING SYSTEM

\begin{tabular}{|c|c|c|c|c|}
\hline $\begin{array}{l}\text { VALVE } \\
\text { NUMBER }\end{array}$ & VALVE NAME & $\begin{array}{l}\text { REQUIRED } \\
\text { POSITION } \\
\end{array}$ & $\begin{array}{c}\text { INITIALS } \\
(1 *) \\
\end{array}$ & $\begin{array}{l}\text { IV } \\
\text { (2) }\end{array}$ \\
\hline HV-AY101EWP-1AI & $\begin{array}{l}\text { Recirc Pump AY101-EW-P-1A } \\
\text { Suction Isolation Valve }\end{array}$ & OPEN & (b) $7 \times$ & ced \\
\hline HV-AY101EWP-1A3 & $\begin{array}{l}\text { Recirc Pump AY101-EW-P-1A } \\
\text { Suction Pressure Gage Isolation }\end{array}$ & OPEN & . TKU & cell \\
\hline HV-AYI01EWP-IA4 & $\begin{array}{l}\text { Recirc Pump AY101-EW-P-IA } \\
\text { Discharge Pressure Gage Isolation }\end{array}$ & OPEN & CW. TKe. & cal \\
\hline HV-AY101EWP-1A5 & $\begin{array}{l}\text { Recirc Pump AY101-EW-P.IA } \\
\text { Discharge Isolation }\end{array}$ & CLOSED & G. & art \\
\hline HV-AY101EWS-IA2 & $\begin{array}{l}\text { Recirc Pump AY10I-EW-P-1A } \\
\text { Discharge Header Isolation }\end{array}$ & OPEN & T.KN & ed \\
\hline$H V \cdot A Y 101 E W P-I B 1$ & $\begin{array}{l}\text { Recirc Pump AY101-EW.P.1B } \\
\text { Suction Isolation Valve }\end{array}$ & OPEN & QU TKW & en \\
\hline HV-AY101EWP-1B3 & $\begin{array}{l}\text { Recirc Pump AY101-EW-P-1B } \\
\text { Suction Pressure Gage Isolation }\end{array}$ & OPEN & $\int_{T K N}$ & ent \\
\hline HV-AY101EWP-1B4 & $\begin{array}{l}\text { Recirc Pump AY101-EW-P-1B } \\
\text { Discharge Pressure Gage Isolation }\end{array}$ & OPEN & TKK & eer \\
\hline HV-AY101EWP-1B5 & $\begin{array}{l}\text { Recirc Pump AY101-EW.P.1B } \\
\text { Discharge Isolation }\end{array}$ & CLOSED & 3. $T K K N$ & Cu \\
\hline HV-AY101-EWS-1A1 & $\begin{array}{l}\text { Recirc Condenser Cooling System } \\
\text { Supply Header Drain Valve in } 241 \text { - } \\
\text { AY-401 valve box. }\end{array}$ & CLOSED & W. $+K N$ & eif \\
\hline$H V \cdot A Y 101 \cdot E W R \cdot I A 1$ & $\begin{array}{l}\text { Recirc Condenser Cooling System } \\
\text { Return Header Drain Valve } 241 \text {-AY- } \\
401 \text { valve box. }\end{array}$ & CLOSED & TKN & cen \\
\hline $\begin{array}{l}\text { HV-AY10I-EWTK- } \\
\text { IAI }\end{array}$ & $\begin{array}{l}\text { Expansion Tank Level Switch LSL- } \\
\text { AY101EWTK-1 Low Side Isolation }\end{array}$ & OPEN & J. T.KN. & \\
\hline $\begin{array}{l}\text { HV-AY101-EWTK. } \\
1 \mathrm{~A} 2\end{array}$ & $\begin{array}{l}\text { Expansion Tank Level Switch LSL. } \\
\text { AY101EWTK-1 High Side Isolation }\end{array}$ & OPEN & (.) Tा TN & at \\
\hline HV-AY101-EWTK-2 & $\begin{array}{l}\text { Recirc Condenser Cooling System Air } \\
\text { Separator Drain Valve }\end{array}$ & CLOSED & KJ. TKN & cut \\
\hline HV-AY101-EWT-1A1 & Cooling Tower Pan Drain Vaive & CLOSED & $T K N$ & and \\
\hline$H V \cdot A Y 101-R W \cdot 1$ & $\begin{array}{l}\text { Cooling Tower Raw Water Isolation } \\
\text { at Evaporator Tower. }\end{array}$ & CLOSED & $7 . K N$ & ed \\
\hline$H V \cdot A Y 10 I W-1$ & $\begin{array}{l}\text { Closed Loop Filtration System Inlet } \\
\text { Block Valve }\end{array}$ & CLOSED & $T K W$ & Bef \\
\hline
\end{tabular}


PREOPERATIONAL TESTING, RECIRCULATION CONDENSER COOLING

HNF-SD-W030-TD-003 REV. 0, PAGE 37

- WHC-SD-W030-POTP-003

Revision No. 0

ATTACHMENT A

PA

Effective Date

TANK AY101 RECIRC CONDENSER COOLING SYSTEM

\begin{tabular}{|c|c|c|c|c|}
\hline $\begin{array}{c}\text { VALVE } \\
\text { NUMBER } \\
\end{array}$ & VALVE NAME & $\begin{array}{l}\text { REQUIRED } \\
\text { POSITION }\end{array}$ & $\begin{array}{l}\text { INITIALS } \\
\left(1^{*}\right)\end{array}$ & $\begin{array}{l}\text { IV } \\
(2)\end{array}$ \\
\hline HV-AY101W-2 & $\begin{array}{l}\text { Closed Loop Filtration System } \\
\text { Recirc Pump AY101-W-P.1 Discharge } \\
\text { Valve }\end{array}$ & $\begin{array}{r}\text { CLOSED } \\
\ddots \quad \nVdash\end{array}$ & $\stackrel{*}{*} / \mathrm{A}$ & $N / A$ \\
\hline$H V-A Y 101 W-3$ & $\begin{array}{l}\text { Closed Loop Filtration System } \\
\text { Outlet Block Valve }\end{array}$ & CLOSED & TKW & $a x$ \\
\hline HV-AY101W-4 & Bag Filter AY101-W-F-2 Inlet Valve & CLOSED & $T K w$ & ent \\
\hline HV-AY101W-5 & Bag Filter AYIOI-W-F-2 Outlet Valve & CLOSED & $71<x$ & $\operatorname{cal}$ \\
\hline$H V-A Y 101 W-6$ & $\begin{array}{l}\text { Conductivity Element inlet } \\
\text { isolation valve }\end{array}$ & OPEN & TKN. & $C$ \\
\hline HV - AY $101 W-7$ & $\begin{array}{l}\text { Conductivity Element outlet } \\
\text { isolation valve }\end{array}$ & OPEN & TKW & $\mu 1$ \\
\hline & & & & \\
\hline
\end{tabular}

Performed

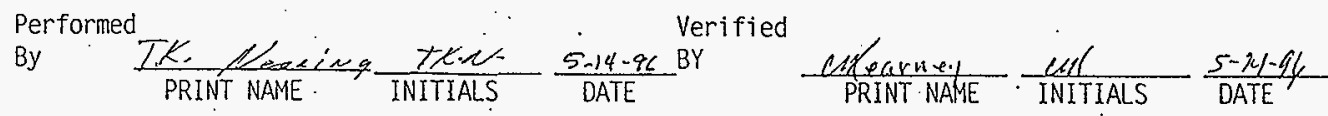

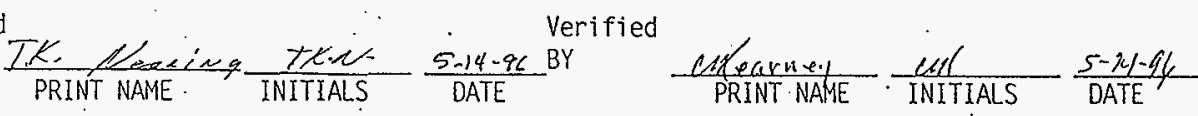

* Valve hV-aylorw-z was. deleter

FROM DESIGN BY ECN-W-030-215,

COPY INCLUDED IN ATT. 3.

NOTE ADDED TO REPORT.

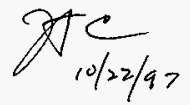




\begin{tabular}{|c|c|c|}
\hline PREOP & $\begin{array}{l}\text { TESTING, RECIRCULATION } \\
\text { WHC-SD-W030-POTP-003 }\end{array}$ & CONDENSER COC \\
\hline Revision No. 0 & $\begin{array}{l}\text { AITACHMENT A } \\
101 \text { RECIRC CONDENSER COOI }\end{array}$ & $\begin{array}{l}\text { Effective } \\
\text { LING SYSTEM }\end{array}$ \\
\hline
\end{tabular}

HNF-SD-W030-TD-003

REV. 0, PAGE 38

TANK AYIOI RECIRC CONDENSER COOLING SYSTEM

APPENDIX C - Tank AY101 Recirc Condenser Cooling System Electrical Alignment

\begin{tabular}{|c|c|c|c|c|}
\hline $\begin{array}{l}\text { BREAKER } \\
\text { NUMBER } \\
\end{array}$ & BREAKER NAME AND LOCATION & $\begin{array}{l}\text { REQUIRED } \\
\text { POSITION } \\
\end{array}$ & $\begin{array}{c}\text { INITIALS } \\
\left(1^{*}\right)\end{array}$ & $\begin{array}{l}\text { IV } \\
(2)\end{array}$ \\
\hline $1 \mathrm{~A}$ & $\begin{array}{l}\text { Evaporator Tower AY101 Supply } \\
\text { Breaker in Distribution PaneIboard } \\
\text { PP-1 Diesel Generator Building }\end{array}$ & ON & $\begin{array}{l}78 \text { Af } \\
\text { TKa }\end{array}$ & ext \\
\hline 2 HP SEPARATOR & $\begin{array}{l}\text { Evaporator Tower AY101 Separator } \\
\text { Recirc Pump Supply Breaker in Local } \\
\text { Panel AY101-EVAP.TWR }\end{array}$ & OFF & 7月7f & cat \\
\hline $\begin{array}{l}\text { FLUID COOLER } \\
\text { CONTROL PANEL } \\
\text { AY101-EW-T-1 }\end{array}$ & $\begin{array}{l}\text { Evaporator Tower AY101 Fan, Recirc } \\
\text { Pump, Pan Heater Supply Breaker in } \\
\text { Local Pane AY101-EVAP.TWR }\end{array}$ & OFF & $\begin{array}{l}7 \& \& 4 \\
T K n\end{array}$ & cent \\
\hline $\begin{array}{l}\text { RECIRC PUMP } \\
\text { AY101-EW-P.1A }\end{array}$ & $\begin{array}{l}\text { AY101 Recirc Condenser Cooling } \\
\text { System Recirc Pump AY101-EW-P-1A in } \\
\text { Local Panel AY101-EVAP-TWR }\end{array}$ & OFF & $\begin{array}{l}7 B \not 2 \\
T / 2 N\end{array}$ & Ut \\
\hline $\begin{array}{l}\text { RECIRC PUMP } \\
\text { AY101-EW-P.1B }\end{array}$ & $\begin{array}{l}\text { AY101 Recirc Condenser Cooling } \\
\text { System Recirc Pump AY101-EW-P-1B in } \\
\text { Local Panel AY101-EVAP-TWR }\end{array}$ & OFF & $\begin{array}{l}7+4 \% \\
T K N\end{array}$ & $\mathscr{C H}$ \\
\hline PANEL PP.7 & $\begin{array}{l}\text { Evaporator Tower Panelboard PP-7 } \\
\text { Supply Breaker in Local Panel } \\
\text { AY101-EVAP-TWR }\end{array}$ & ON & $\begin{array}{l}\text { TAP } \\
\text { TKW }\end{array}$ & \\
\hline $\begin{array}{l}\text { Disconnect } \\
\text { switch }\end{array}$ & $\begin{array}{l}\text { Evaporator Tower AY101 Separator } \\
\text { Recirc Pump local disconnect switch } \\
\text { at pump. }\end{array}$ & OFF & $\begin{array}{l}\text { Poff } \\
\text { TKW }\end{array}$ & \\
\hline & & & & \\
\hline & & & & \\
\hline & & & & \\
\hline & & & & \\
\hline & & & & \\
\hline & & & & \\
\hline
\end{tabular}

'erformed

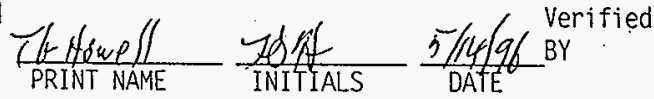

Euleanary 


PREOPERATIONAL TESTING, RECIRCULATION CONDENSER COOLING
WHC-SO-W030-POTP.003
ATTACHMENT A

Equipment Name: IANK AYIOL RECIRC CONDENSER COOLING SYSTEM RECIRC PUMP

EQUIPMENT I.0. NO. : AY101-EW:P-IA

NAMEPLATE DATA: ___ See Appendix F. Jo

VIBRATION DATA (mil)

\begin{tabular}{|l|l|l|l|l|}
\hline IST READING & $\begin{array}{l}\text { MOTOR } \\
\text { OUTBOARO }\end{array}$ & $\begin{array}{l}\text { MOTOR } \\
\text { INBOARD }\end{array}$ & $\begin{array}{l}\text { PUMP } \\
\text { INBOARO }\end{array}$ & $\begin{array}{l}\text { PUMP } \\
\text { OUTBOARD }\end{array}$ \\
\hline HORIZONTAL & & & \\
\hline VERTICAL & See & rst Repert, Att. & 3 \\
\hline AXIAL & Typical all data sheets. \\
\hline
\end{tabular}

\begin{tabular}{|l|l|l|l|l|}
\hline 2ND READING & $\begin{array}{l}\text { MOTOR } \\
\text { OUTBOARD }\end{array}$ & $\begin{array}{l}\text { MOTOR } \\
\text { INBOARD }\end{array}$ & $\begin{array}{l}\text { PUMP } \\
\text { INBOARD }\end{array}$ & $\begin{array}{l}\text { PUMP } \\
\text { OUTBOARD }\end{array}$ \\
\hline HORIZONTAL & & & & \\
\hline VERTICAL & DiTto & & & \\
\hline AXIAL & & & & \\
\hline
\end{tabular}

\begin{tabular}{|l|l|l|l|l|}
\hline $\begin{array}{l}\text { PUMP BEARING } \\
\text { TEMPERATURES }\end{array}$ & $\begin{array}{l}\text { MOTOR } \\
\text { OUTBOARD }\end{array}$ & $\begin{array}{c}\text { MOTOR } \\
\text { INBOARD }\end{array}$ & $\begin{array}{l}\text { PUMP } \\
\text { OUTBOARD }\end{array}$ & PUMP INBOARD \\
\cline { 2 - 5 } & & & & \\
\hline
\end{tabular}

REMARKS:

Performed by :

Date

Verified by :

Date 


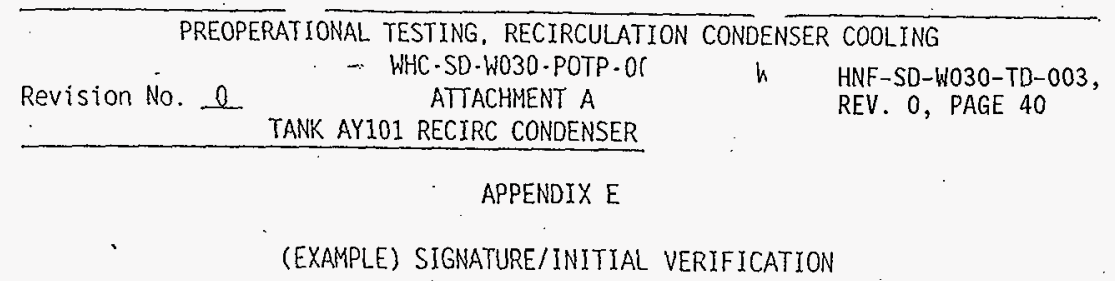

All persons involved in procedure performance. data recording. and verification or evaluation of test steps shall provide their name. job title. signature, and initials in the following table.

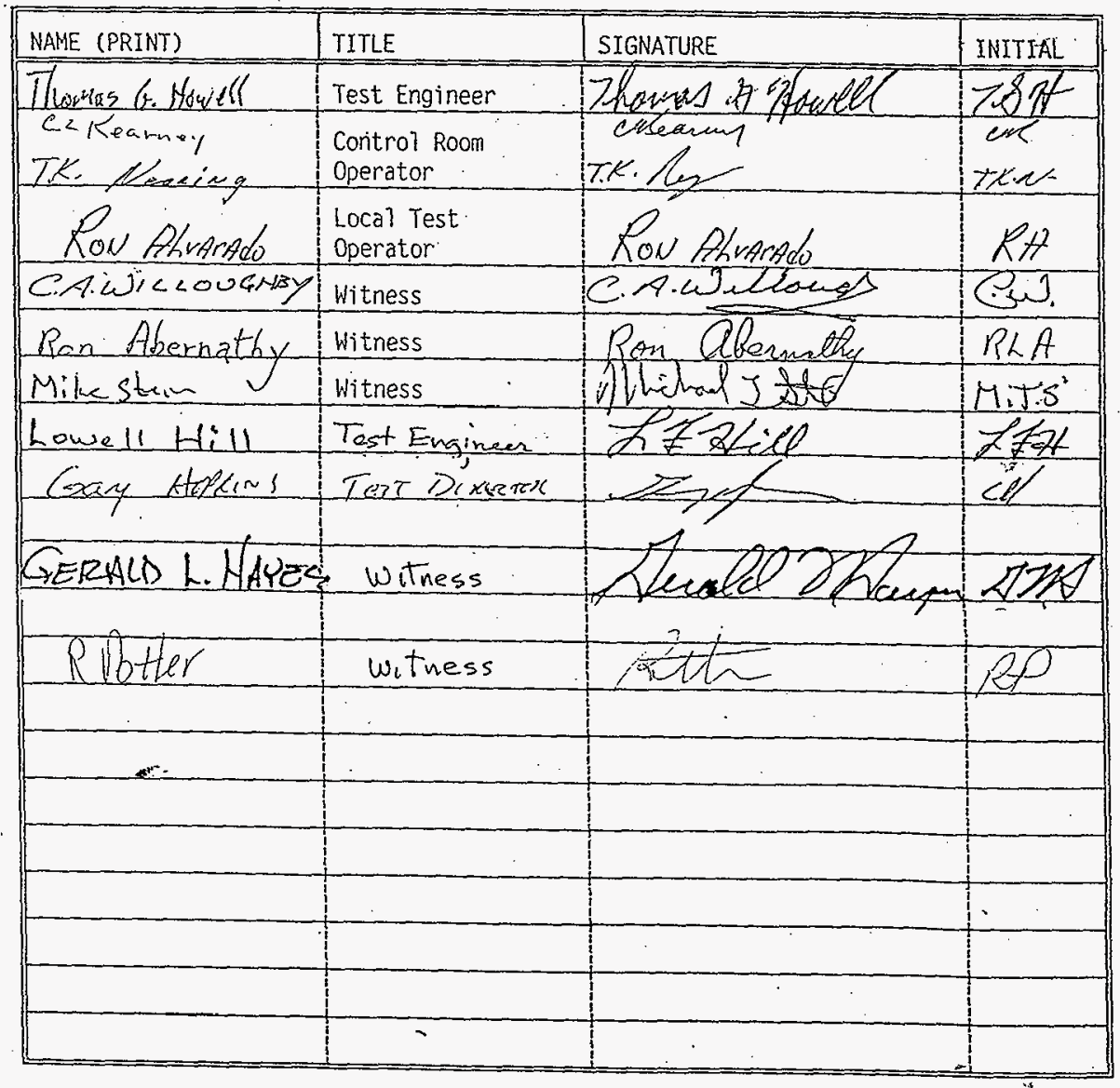




\begin{tabular}{ccc}
\hline PREOPERATIONAL TESTING, RECIRCULATION CONDENSER COOLING \\
WHC-SO-WO30-POTP-003 & HNF-SD-W030-TD-003, \\
ATTACHMENT A & REV. 0, PAGE 41 \\
Revision No. 0 & TANK AYIOI RECIRC CONDENSER C &
\end{tabular}

APPENDIX F

\begin{tabular}{|c|c|c|}
\hline TEST LOG & $\begin{array}{l}\text { FEST NUYBER: } \\
\text { WHC-SO-WOZ- } \\
\text { POTA- - SOS }\end{array}$ & $\begin{array}{l}\text { TEST LOS } \\
\text { PAGE NUMBER: } \\
t \\
\end{array}$ \\
\hline
\end{tabular}

TEST MITLE:

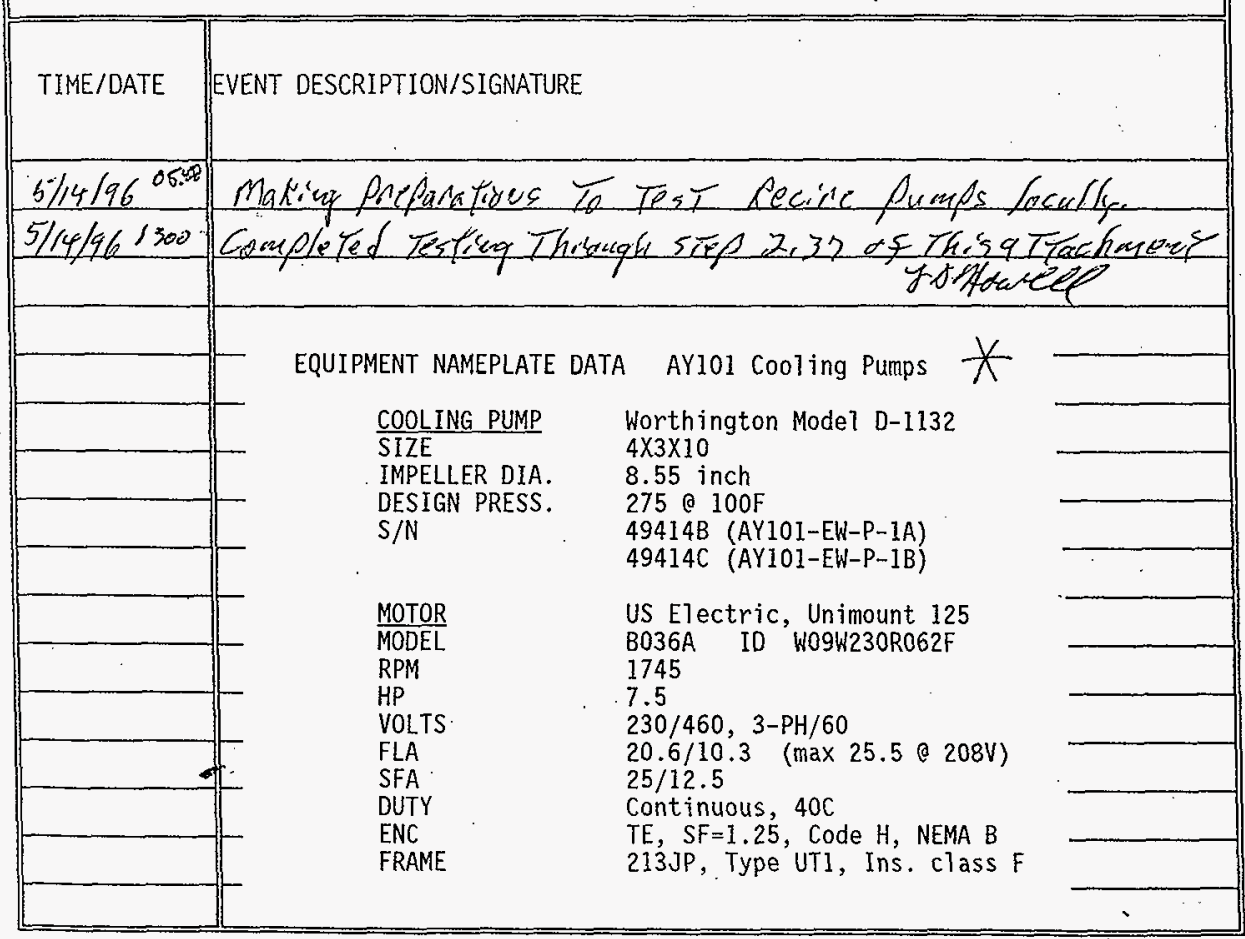

* Date added to iest Report De 
PREOPER .ONAL TESTING, RECIRCULATION CON. NSER COOLING WHC-SD-W030-POTP-003

Revision No. 0

ATTACHETT D

HNF-SD-W030-TD-003, REV. 0, PAGE 42

TANK AZ102 RECIRC CONDENSER COOL

AYIOI

APPENDIX $F$

TEST LOG

TEST TITLE:

TIME/DATE

EVENT DESCRIPTION/SIGNATURE

EQUIP

\section{NAMEPLAT}

DATA

AY101 COOLING TOWER

\section{X}

TOWER Baltimore Aircoil (BAC)

MODEL

$S / N$

F1-742-K.

BELT

94200707

$\mathrm{B} 73$

PUMPS

MODEL

SPRAY PUMP

SIZE

IMP. DIA.

BAC \#300211

$S / N$

$150 \mathrm{GPM}, \mathrm{TDH} 12 \mathrm{ft}$

PJM032394E

TYPE

--

MOTOR

$S / N$

MODEL

RPM

HP

VOLTS

FLA

SFA

DUTY

ENC

FRAME
General Electric, XHL210590

5K38PN48 (cat K221)

3450

1

208-230/460, 3-PH/60

$3.3-3.2 / 1.6$

4.7-4.4/2.2

Continuous, $40 \mathrm{C}$

$S F=1.4$, Code $M$

Ins. class $B$

$56 \mathrm{~J}$
FILTER PUMP

Grundfos 89404

85 GPM, TDH $61 \mathrm{ft}$ 03500RPM $-$

EPI50-2020-U-B-A-BBVV

Ba7dor Cat. \#VM3555-NP

F1293

Spec. \#35A13-672F5

3450

2

208-230/460

$5.7-5.4 / 2.7$

$--$

Continuous, $40 \mathrm{C}$

TE, $S F=1.15$, Code $H$

Ins. class $B$

$56 \mathrm{C}$ of 


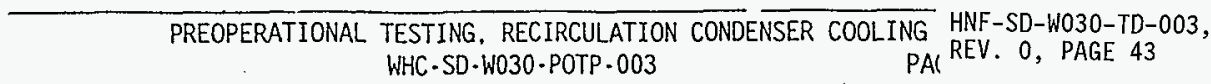

Revision No. e

ATTACHMENT A

Effective Di..

TANK AY101 RECIRC CONDENSER COOLING SYSTEM

\section{APPENDIX G}

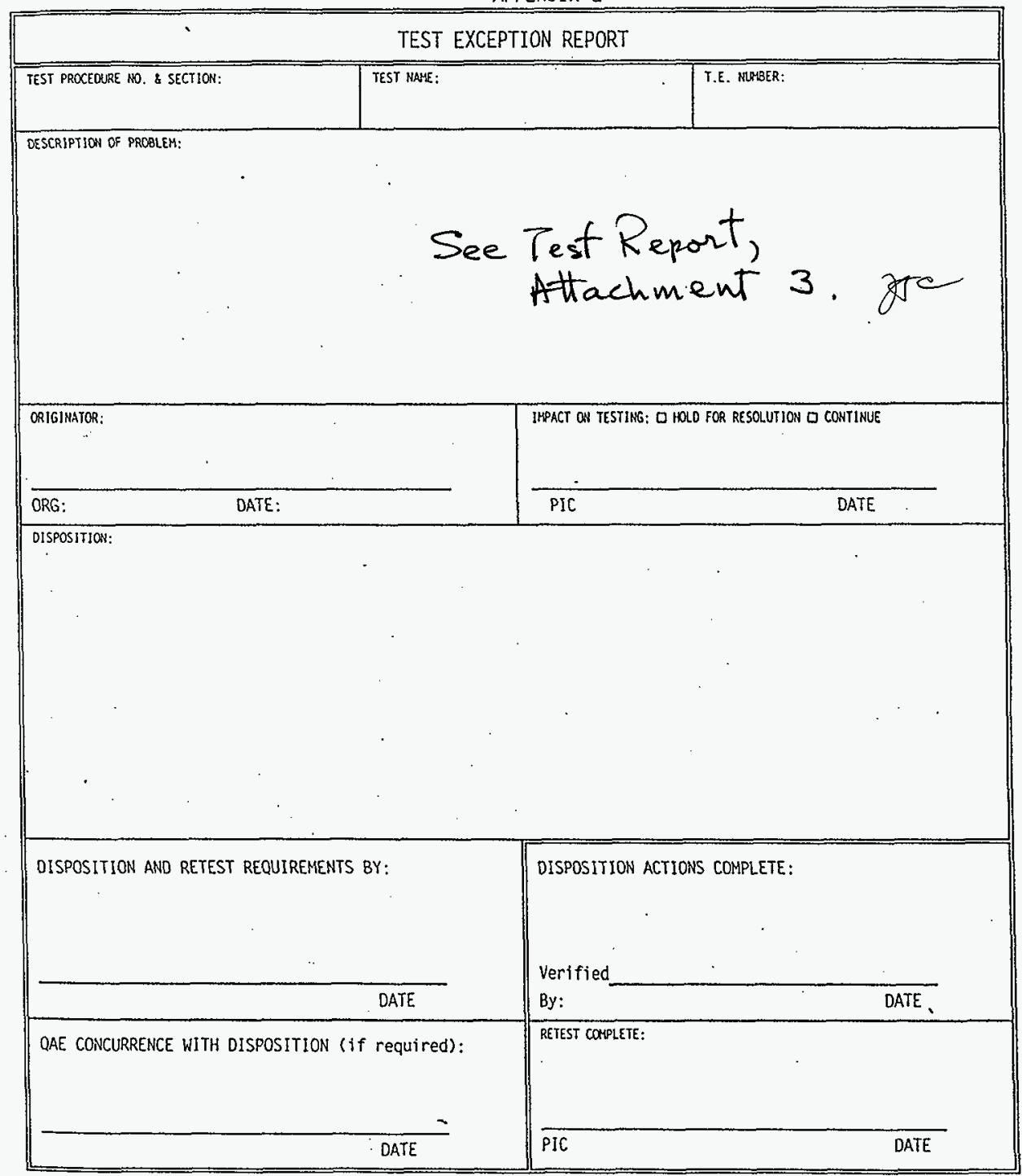




\begin{tabular}{c} 
PREOPERAT LONAL TESTING, RECIRCULATION INNNFNC \\
WHC-SD.WO30.POTP-003 \\
ATTACHMENT B \\
Revision No. \& \\
TANK AY102 RECIRC CONDENSER Cl \\
\hline
\end{tabular}

\subsection{INITIAL CONDITIONS}

1.1 VERIFY all system instrumentation in Appendix $A$ is calibrated and has a current calibration tag affixed to each instrument and that a)l loop calibrations are complete.

Test Engineer:

HNF-SD-W030-TD-003, REV. 0, PAGE 44

1.2 VERIFY the system is aligned for preoperational testing in accordance with Appendix $B$.

Test Engineer:

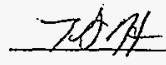

1.3 VERIFY system electrical circuit breakers are aligned in accordance with Appendix C.

Test Engineer:

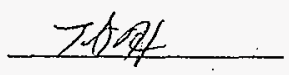

1.4 VERIFY expansion tank leve] is at midlevel as indicated on LIAY102EWTK-1.

Test Engineer:

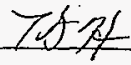

1.5 All personnel who will be involved with this procedure have provided the required signature verification information in Appendix E .

Test Engineer:

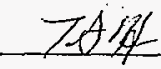

1.6 The test engineer has verified, by review of the tag $\log$ and a walkdown of the systems being tested, that all components within and including the test boundary have been "blue" tagged.

Test Engineer:

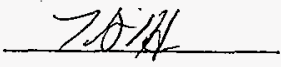

2.0 Tank AY102 Glycol Recirc System Testing

2.1 VERIFY Recirc Pump AY102-EW-P-1A local control switch HS-AY102EWPIAI in the STOP pOsition.

Test Engineer:

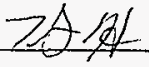

2.2 CLOSE local breaker for Recirc Pump AY102-EW-P-IA. 
PREOPERATIONAL TESTING. RECIRCULATION CONDENSER COOLING WHC-SO-WO3O-POTP-OC

Revision No. 0

ATTACHMENT B

HNF-SD-W030-TD-003,

REV. 0 , PAGE 45

TANK AY102 RECIRC CONDENSER

2.3 RECORD the following data for Recirc Pump AY102-EW-P-1A:

Expansion Tank Level (Local)

Suction Pressure(Local)

8 psi

Run Time (graphics screen 06EvpAY1.v) \& hrs

Test Engineer:

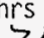

2.4 ATTEMPT to start Recirc Pump AY102-EW-P-1A from MCS graphics screen 06EvpAY1.v.

2.5 VERIFY Recirc Pump AY102-EW-P-1A does not start.

Test Engineer: To\&

2.6 BUMP Recirc Pump AY102-EW-P-1A by placing control switch HSAY102EWP-IA1 in START and then back to STOP.

2.7 VERIFY rotation of the Recirc Pump AY102-EW-P-IA is in the direction indicated by the arrow on the pump.

Test Engineer:

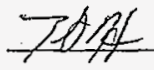

2.8 PLACE Recirc Pump AY102-EW-P-1A local control switch HS-AY102EWP$1 A I$ in the START position.

2.9 VERIFY Recirc Pump AY102-EW-P-1A starts.

Test Engineer:

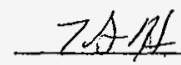

2.10 RECORD the following data for Recirc Pump AY102-EW-P-1A:

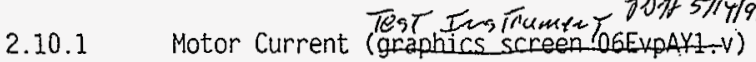

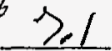
Amps

2.10 .2

2.10 .3

Motor Voltage (TEST INST.) 492 volts

Suction Pressure (LOCAL) psi

ar. $\quad 2.10 .4$

Discharge Pressure (LOCAL)

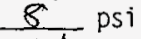

Test Engineer: psi

2.11 SLOWLY OPEN Recirc pump AY102-EW-P-1A discharge valve HV-AYI02EWP$1 A 5$ and establish a system flowrate of $2192 \mathrm{gpm}$ as indicated on FI-AY102EWR-1 on graphics screen 06EvpAY1.v. 


\begin{tabular}{c}
\hline PREOPERATIONAL TESTING, RECIRCULATION CONDENSER COOLING \\
WHC-SD-WO30-POTP-00: \\
ATTACHMENT B
\end{tabular}

2.12 VERIFY low flow alarm FAL-AY102WR-1 clears graphics screen 06EvpAY1.V.

Test Engineer:

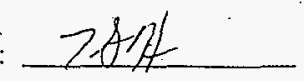

2.13 RECORD the following data for Recirc Pump AY102-EW-P-1A:

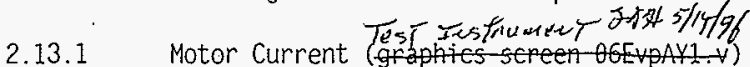

95

2.13.2 Motor Voltage (TEST INST.) $49 \mathrm{z}$ Volts

2.13.3 Suction Pressure (LOCAL) _

2.13.4 Discharge Pressure (LOCAL) 42 psi

2.13.5 System Flow (graphics screen 06EvpAY1.v) $195 \mathrm{gpm}$

2.13.6 Expansion Tank Level (Local) Y/2

Test Engineer:

2.14 THROTTLE CLOSED VaTve HV-AY102EWP-1A2 until system flow starts to decrease as indicated on FI-AY102EWR-1 graphics screen 06EvpAY1.v.

2.15 FULLY OPEN Recirc Pump AY102-EW-P-IA discharge valve HV-AY102EWP-. $1 \mathrm{A5}$.

2.16 ADJUST system flow to $z 192 \mathrm{gpm}$ using HV-AY102EWP-1A2

2.17 VERIFY Recirc PUmp AY102-EW-P-1B local control switch HS-AY102EWP$1 B 1$ in the STOP pOsition.

2.18 CLOSE local breaker for Recirc Pump AY102-EW-P-1B.

2.19 RECORD the following data for Recirc Pump AY102-EW-P-1B:

Suction Pressure(Local) $\gg .5$ psi

Run Time (graphics screen 06EvpAYl.v) $O$ hrs.

Test Engineer:

zity

2.20 BUMP Recirc Pump AY102-EW-P-1B by placing control switch HSAY102EWP-1BI in START and then back to STOP.

2.21 VERIFY rotation of the Recirc Pump AY102-EW-P-1B is in the direction indicated by the arrow on the pump.

Test Engineer:

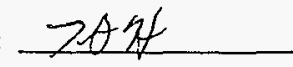




PREOPERATIONAL TESTING, RECIRCULATION CONDENSER COOLING
WHC-SD-WO30-POTP-OC
TANK AY102 RECIRC CONDENSER

2.22 ATTEMPT to start Recirc Pump AY102-EW-P-1B from MCS graphics screen 06EvpAY1.v.

2.23 VERIFY Recirc Pump AY102-EW-P-1B doès not start.

\section{Test Engineer: ZAZK}

2.24 PLACE Recirc Pump AY102-EW-P-1B local control switch HS-AY102EWP$1 B 1$ in the START position.

2.25 VERIFY Recirc Pump AY102-EW-P-1B starts. Test Engineer: 7AA 2h

2.26 RECORD the following data for Recirc Pump AY102-EW-P-1B:

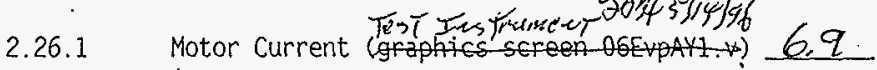

2.26.2 Motor voltage (TEST INST.) 492 volts

2.26.3 Suction Pressure (LOCAL) $\frac{4.5}{25 i}$

2.26.4 Discharge Pressure (LOCAL) 4 _t psi Test Engineer:

2.27 SLOWLY OPEN Recirc pump AY102-EW-P-1B discharge valve: HV-AYI02EWP$1 \mathrm{B5}$.

2.28 ATTEMPT to stop Recirc Pump AY102-EW-P-1A from MCS graphics screen 06EvpAY1.V.

2.29 VERIFY that Recirc Pump AY102-EW-P-1A does not STOP.

Test Engineer:

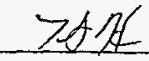

2.30 PLACE Recirc. Pump AY102-EW-P-1A local control switch HS-AY102EWPIAl in the STOP pOsition.

2.31 VERIFY, that Recirc Pump AY102-EW-P-1A STOPS.

Test Engineer: ZI/f

2.32 ADJUST system flow, if required, to $2192 \mathrm{gpm}$ using HV-AY102EWP. 1 A2. 
PREOPERATIONAL TESTING, RECIRCULATION CONDENSER COOLING WHC-SD-W030-POTP- 0

Revision No. 0 ATTACHMENT B TANK AY102 RECIRC CONDENSER

HNF-SD-W030-TD-003,

REV. 0, PAGE 48

2.33 RECORD the following data for Recirc Pump AY102-EW-P-1B:

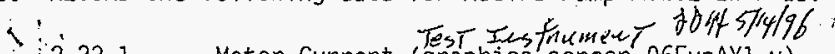

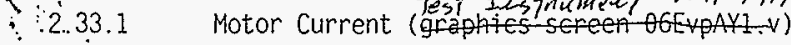

9.4

2.33 .2 Amps

2.33 .3 Motor voltage (TEST INST.)

2.33 .4 Suction Pressure (LOCAL)

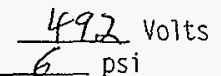

2.33 .5

Discharge Pressure (LOCAL)

2.33 .6

System Flow (graphics screen 06EvpAYI.v) $193 \mathrm{gpm}$ Expansion Tank Leve] (Local)

Test Engineer:

$Y_{2}$

rAft

2.34 THROTTLE HV-AY102EWP-1A2 to decrease flow to $<170 \mathrm{gpm}$.

2.35 VERIFY system low flow a Tarm, FAL-AY102EWR-1 annunciates on graphics screen 06EvpAY1.v.

$\because \cdots$

Test Engineer:

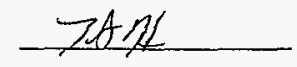

2.36 THROTLE HV-AY102EWP-IA2 to increase flow to $2192 \mathrm{gpm}$.

2.37 VERIFY system low flow alarm. FAL-AY102EWR-1 clears on graphics screen 06EvpAYI.v.

Test Engineer:

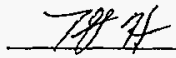

2.38 PLACE Recirc Pump AY102-EW-P-IA local control switch HS-AY102EWPIAl in the REMOTE position.

2.39 START Recirc Pump AY102-EW-P-1A from graphics screen 06EvpAY1.v

2.40 VERIFY Recirc Pump AY102-EW-P-1A starts.

Test Engineer:

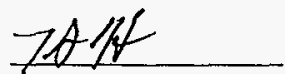

2. 41 PLACE Recirc Pump AY102-EW-P-1B local control switch HS-AY102EWP$1 B 1$ in the STOP position.

2.42 VERIFY Recirc Pump AY102-EW-P-IB stops.

Test Engineer:

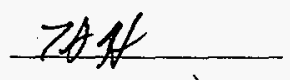

2.43 PLACE Recirc Pump AY102-EW-P-1B locàl control switch HS-AY102EWP181 in the REMOTE position.

2.44 PLACE Recirc Pump AY102-EW-P-1B in Standby on graphics screen OGEvPAYl.V. 
PREOPERATIONAL TESTING, RECIRCULATION CONDENSER COOLING WHC-SD-W030-POTP-003

Revision No. 2 ATTACHMENT B

HNF-SD-W030-TD-003, REV. O, PAGE 49

TANK AY102 RECIRC CONDENSER CI

2.45 OPEN local breaker switch for Recirc Pump AY102-EW-P-IA.

2.46 VERIFY Recirc Pump.AY102-EW-P-1B auto starts.

Test Engineer:

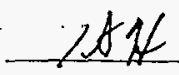

2.47 PLACE Recirc Pump AY102-EW-P-IA remote control switch HS-AY102EWP$1 A$ in STOP position on graphics screen 06EvpAYl.v.

2.48 CLOSE local breaker for Recirc Pump AY102-EW-P-1A.

2.49 PLACE Recirc Pump AY102-EW-P-1A in the Standby on graphïs screen 06EvPAYl.v.

2.50 OPEN local breaker for Recirc Pump AY102-EW-P-1B.

2.51 VERIFY Recirc Pump AY102-EW-P-1A auto starts. Test Engineer:

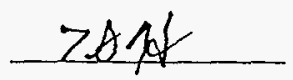

2.52 PLACE Recirc Pump AY102-EW-P-IB remote control switch HS-AY102EWP$1 B$ in STOP position on graphics screen 06EvPAY1.v.

2.53 CLOSE local breaker for Recirc Pump AY102-EW-P-1B.

2.54 ALLOW Recirc Pump AY102-EW-P-1A to operate for one hour for collection of vibration data and bearing data.

2.55 RECORD vibration data and bearing temperatures on Appendix D-1.

Test Engineer:

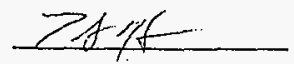

2.56. START Recirc Pump AY102-EW-P-18 remotely from LCU4-17.

2.57 STOP Recirc Pump AY102-EW-P-IA remotely from LCU4-16.

2.58 ALLOW Recirc Pump AY102-EW-P-1B to operate for one hour for collection of vibration data and bearing data.

2.59 "RECORD vibration data and bearing temperatures on Appendix D-2.

Test Engineer:

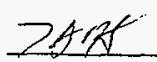




\begin{tabular}{ccc} 
PREOPERA. JNAL TESTING. RECIRCULATION CONIIINSFR COOI TNG & WHC-SD-W030-POTP-00 \\
ATTACHMENT B & HNF-SD-W030-TD-003, \\
Revision No. 0 & REV. 0, PAGE 50 \\
\hline
\end{tabular}

2.60 SLOWLY CLOSE Expansion Tank Leve? Switch LSL-AY102EWTK- 1 Low Side Isolation Valve HV-AY102EWTK-IAI.

2.61 VERIFY Expansion Tank LOW Level ATarm LAL-AYI02EWTK-1 annunciates on graphics screen 06EvpAY1.V.

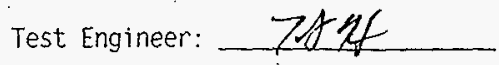

2.62 OPEN Expansion Tank Level Switch LSL-AY102EWTK- 1 Low Side Isolation Valve HV-AY102EWTK-1A1.

2.63 VERIFY Expansion Tank Low Level Alarm LAL-AY102EWTK-1 clears on -graphics screen 06EvpAY1.v.

$\because \therefore$

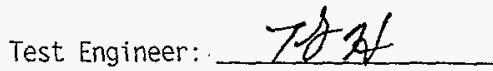

2.64 PLACE Low Flow Shutdown switches HS-AY102EWP-1AB and HS-AY102EWPIBB in the OVERRIOE position on graphics screen O6EVPAY1.V.

2.65 PLACE Recirc Pump AY102-EW-P-1A in STANOBY on graphics screen 06EvpAY1.V.

2.66 THROTTLE HV-AY102EWP-1A2 to decrease flow to $<100 \mathrm{gpm}$.

2.67 VERIFY the Recirc Pump AY102-EW-P-1B does not STOP on Tow flow.

Test Engineer:

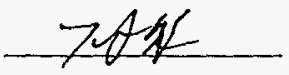

2.68 PLACE LOW Flow Shutdown Override switch HS-AY102EWP-1BB in the NORMAL pOsition from the LOI.

2.69. VERIFY the Recirc Pump AY102-EW-P-1B STOPS on low flow.

Test Engineer:

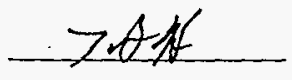

2.70 VERIFY Recirc Pump AY102-EW-P-1A starts.

Test Engineer:

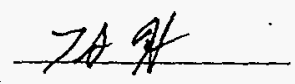

2.71 PLACE LOW Flow Shutdown Override switch HS-AY102EWP-IAB in the NORMAL position on graphics screen 06EvpAY1.V.

2.72 VERIFY the Recirc Pump AY102-EW-P-1A STOPS on low flow.

Test Engineer:

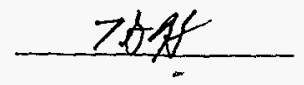




PREOPERATIONAL TESTING, RECIRCULATION CONDENSER COOLING
WHC-SD-W030-POTP-00
ATTACHMENT B
TANK AY102 RECIRC CONDENSER

2.73 PLACE LOW Flow Shutdown Override switch HS-AY102EWP-1AB in the OVERRIOE position on graphics screen O6EVPAYI.V.

2.74 RESET Recirc Pump AY102-EW-P-1A by placing remote control switch HS-AY102EWP-IA in STOP position on graphics screen O6EvpAY1.V. $\because$.

2.75 PLACE Recirc Pump AY102-EW-P-1A remote control switch HS-AY102EWP$1 A$ in START position on graphics screen 06EvpAY1.v.

2.76 VERIFY that Recirc Pump AY102-EW-P-IA starts.

$\because \quad \therefore \quad$

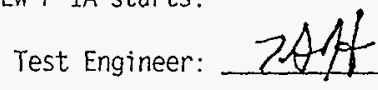

2.77 . THROTTLE HV-AY102EWP-1A2 to increase system flow to $\geq 192 \mathrm{gPm}$.

2.78 PLACE LOW Flow Shutdown Override switch HS-AY102EWP-1AB in the NORMAL position on graphics screen 06EvPAY1.V.

3.0 Tank AY102 Evaporator Tower Testing

3.1 REMOVE manway covers on the cooling tower.

3.2 OPEN HV-AY102-RW-1 Cooling Tower Raw Water Isolation valve at Evaporator Tower.

3.3 'VERIFY' cooling tower fill via makeup valve EV-AYI02EWT-1AI.

Test Engineer:

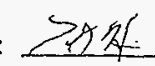

3.4 INSTALL manway covers when cooling tower fill is complete.

3.5. VERIFY..the following local switch positions:

- Evaporator Tower AY102-EW-T-1 Spray Pump AY102-EW-SP-1 Toca1,

ar. control switch HS-AY102-EWSP-1A in OFF:

$\because$ : Evaporator Tower AY102-EW-T-I Fan 7ocal control switch HSAY102EWT-1A1 in OFF.

- Evaporator Tower AY102-EW-T-1 Pan Heater local control switch HS-AY102EWT-1A 2 in OFF.

Test Engineer: $7 \not 14$

3.6 CLOSE the local supply breaker for the spray pump. cooling fan, and pan heater in the Evaporator Tower local distribution panel. 


\begin{tabular}{ccc}
\hline PREOPER & $\begin{array}{c}\text { ONAL TESTING, RECIRCULATION CONL NSER COOLING } \\
\text { WHC-SO-W030-POTP-003 }\end{array}$ \\
Revision No. 0 & ATTACHMENT B & HNF-SD-W030-TD-003, \\
& TANK AY102 RECIRC CONDENSER C & REV. 0, PAGE 52
\end{tabular}

3.7 REMOVE splash guard from top of Spray Pump AY102-EW-SP-1 motor to enable viewing of the motor shaft.

3.8 BUMP Spray Pump AY102-EW-SP-1 by placing control switch HS-AY102EWSP-1A in START and then back to OFF.

3.9 VERIFY rotation of the Spray Pump AY102-EW-SP-1 is in the direction indicated by the arrow on the pump.

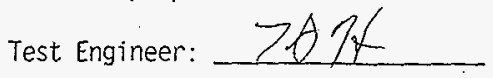

3.10 REINSTALL splash guard on Spray Pump AY102-EW-SP-1 motor.

3.11 PLACE Evaporator Tower AY102-EW-T-1 Spray Pump AY102-EW-SP-1 loca1 control switch HS-AY102-EWSP-1A in START.

3.12 VERIFY Spray Pump AY102-EW-SP-1 starts.

Test Engineer:

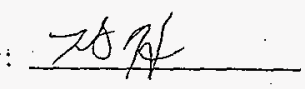

3.13 RECORD the following data for Spray Pump AY102-EW-SP-1:

3.13.1 Motor Current (TEST INST.) 1,5 Amps

3.13.2 Motor Voltage (TEST INST.) 486 Volts

3.13.3 Spray Flow (TEST INST.) NIA gpm

Test Engineer:

3.14 ALLOW Spray Pump AY102-EW-SP-1 to operate for one hour for collection of vibration data and bearing data.

3.15 RECORD vibration data and bearing temperatures on Appendix D-3.

Test Engineer:

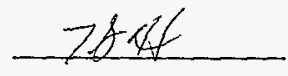

3.16 PLACE Evaporator Tower AY102-EW-T-1 Spray Pump AY102-EW-SP-1 remote control switch HS-AY102-EWSP-1 in STOP on graphics screen 06EvpAY1.v.

3.17 VERIFY Spray Pump AY102-EW-SP-1 does NOT STOP. Test Engineer:

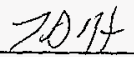

3.18 PLACE Evaporator Tower AY102-EW-T-1 Temperature Control Damper local control Switch HS-AY102-EWT-1A3 in OPEN. 


\section{PREOPERAT, IAL TESTING, RECIRCULATION CONDENSER COOLING WHC-SD-W03O-POTP- 0 \\ Revision No. O ATTACHMENT B TANK AY102 RECIRC CONDENSER \\ HNF-SD-W030-TD-003, REV. O, PAGE 53}

3.19 VERIFY Evaporator Tower AY102-EW-T-1 Temperature Control Damper OPENS.

Test Engineer:

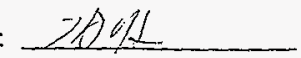

3.20 PLACE Evaporator Tower AY102-EW-T-1 Cooling Fan local control switch HS-AY102-EWT-1A1 in START.

3.21 VERIFY Evaporator Tower AY102-EW-T-1 Cooling Fan starts.

Test Engineer:

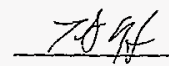

3.22 RECORD the following data for Evaporator Tower AY102-EW-T-1 Cooling Fan:

3.22.1. Motor Current (TEST INST.) 10. 7 Amps

3.22.2 Motor Voltage (TEST INST.) 483 volts

Test Engineer: $Z 27 f$

3:23 ALLOW Evaporator Tower AY102-EW-T-1 Cooling Fan to operate for one hour for collection of vibration data and bearing data.

3.24 RECORD vibration data and bearing temperatures on Appendix D-4.

Test Engineer:

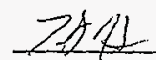

3.25 PLACE Evaporator Tower AY102-EW-T-1 Cooling Fan remote control switch HS-AY102-EWT-1 in OFF on graphics screen 06EvpAY1.v.

3.26 VERIFY Evaporator Tower AY102-EW-T-1 Cooling Fan does NOT STOP..

Test Engineer: YAR

3.27 PLACE Evaporator Tower AY102-EW-T-I Pan Heater local control switch HS-AY102-EWT-1A2 in ON.

3.28 VERIFY Evaporator Tower AY102-EW-T-1 Pan Heater remains deenergized by checking the heater contactor is OPEN.

3.29 SIMULATE a low Evaporator Tower Pan level.

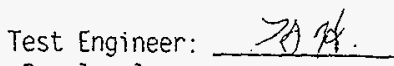


PREOPERATIONAL TESTING, RECIRCULATION CONDENSER COOLING WHC-SD.W030-POTP.003

Revision No. O ATTACHMENT B TANK AY102 RECIRC CONDENSER C .

HNF-SD-W030-TD-003, REV. 0, PAGE 54

3.30 VERIFY the following actions occur:

3.30.1 Evaporator Tower Spray Pump AY102-EW-SP-1 stops.

3.30.2 Evaporator Tower Cooling Fan stops.

3.30.3 Evaporator Tower Makeup Và7ve EV-AY102EWT-1A1 opens.

Test Engineer: $20 \%$

3.31 RECORD makeup flow at FI-AZRW-1 (graphics screen 05water.v). $20.9 p m$.

Test Engineer:

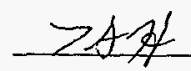

3.32 RESTORE the Evaporator Tower Pan level to normal.

3.33 VERIFY Evaporator Tower Pan Heater energizes by checking the heater contactor is CLOSED.

Test Engineer:

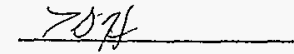

3.34 PLACE Evaporator Tower AY102-EW-T-1 Temperature Control Damper local control Switch HS-AY102-EWT-1A3 in AUTO.

3.35 PLACE Evaporator Tower AY102-EW-T-1 Spray Pump AY102-EW-SP-1 local control switch HS-AY102-EWSP-1A in REMOTE.

3.36 PLACE Evaporator Tower AY102-EW-T-1 Cooling Fan local control switch HS-AY102-EWT-1AI in REMOTE.

3.37 PLACE Evaporator Tower AY102-EW-T-1 Spray Pump AY102-EW-SP-1 remote control switch HS-AY102-EWSP-1 in START on graphics screen 06EvpAYl.v.

3.38 VERIFY Evaporator Tower AY102-EW-T-1 Spray Pump AY102-EW-SP-1 starts.

Test Engineer: " $D$ LL

3.39 VERIFY Evaporator Tower Pan Heater deenergizes by checking the heater contactor is OPEN.

Test Engineer:

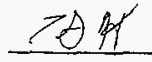

3.40 PLACE Evaporator Tower AY102-EW-T-I remote control switch HSAY102-EWT-1AI in ON on graphics screen 06EvpAY1.v. 
PREOPERATI NAL TESTING, RECIRCULATION CONDENSER COOLING WHC - SO - WO3O - POTP - C

Revision No. 0 ATTACHMENT B TANK AY102 RECIRC CONDENSEK

HNF-SD-W030-TD-003, REV. 0, PAGE 55

3.41 VERIFY the following events occur:

3.41.1 AY102 Evaporator Tower Cooling Fan starts.

3.41.2 AY102 Evaporator Tower AY102-EW-T-1 Temperature

Control Damper modulates to maintain glycol

temperature at setpoint $100^{\circ} \mathrm{F}$ as indicated on graphics screen06EvpAY1.y.

Test Engineer:

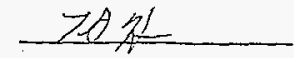

3.42 OPEN Closed Loop Filtration System inlet and outlet block valves HV-AY102W-I and HV-AY102W-3.

3.43 BUMP the Closed Loop Filtration System Recirc Pump AY102-W-P-1 by placing control switch HS-AYI02WP-I in HAND and then back to OFF.

3.44 VERIFY rotation of the Closed Loop Filtration System Recirc Pump AY102-W-P-1 is in the direction indicated by the arrow on the pump.

Test Engineer:

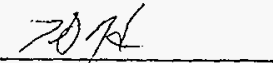

3.45 PLACE the Closed Loop Filtration System Recirc Pump AY102-W-P-1 local control switch AY102WP-1 in HAND.

3.46 VERIFY Closed Loop Filtration Recirc Pump AY102-W-P-1 starts.

Test Engineer:

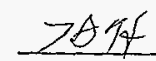

3.47 SLOWLY OPEN Closed Loop Filtration System Recirc Pump AY102-W-P-1. discharge valve HV-AY102W-2 to establish full system flow.

3.48 RECORD the following data for Closed Loop Filtration Recirc Pump AY102-W-P-1:

3.48.1 Motor Current (TEST INST.) 2, 2 Amps

3.48.2 Motor Voltage (TEST INST.) 482 Volts

3.48 .3

3.48 .4

Recirc Flow (TEST INST.) N NA gpm

3.48 .5

Separator Inlet Pressure (LOCAL) $\geq 0$ psi

Separator Outlet Pressure (LOCAL)

Test Engineer: psi

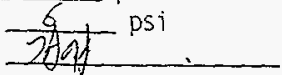

3.49 ALLOW Closed Loop Filtration Recirc Pump AY102-W-P-1 to operate for one hour for collection of vibration data and bearing data. 
PREOPERAIIONAL TESTING, RECIRCULATION CONDENSER COOLING WHC-SD-WO3O-POTP-OO'

Revision No. 0 ATTACHMENT B TANK AY102 RECIRC CONDENSER !

HNF-SD-W030-TD-003, REV. 0, PAGE 56

3.50 RECORD vibration data and bearing temperatures on Appendix D-5.

Test Engineer:

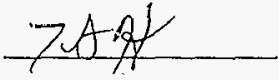

3.51 OPEN Bag Filter AY102-W-F-2 inlet isolation valve HV-AY102W-4.

3.52 VENT all air from the filter housing using the pressure/vacuum relief valve on top of the filter.

3.53 OPEN Bag Filter AYI02-W-F-2 outlet isolation valve HV-AY102W-5.

3.54 VERIFY bag filter differential pressure indicates in the green range on PDIS-AYI02WF-2.

Test Engineer: $>A \lambda d$

3.55 LOOSEN fitting on Bag Filter PDIS-AY102WF-2 low side instrument tubing.

3.56 VERIFY Bag Filter high differential pressure alarm PDAH-AY102WF-2 initiates at 15psid on graphics screen 06EvpAYl.v.

Test Engineer:

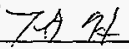

3.57 TIGHTEN fitting Bag Filter PDIS-AY102WF-2 low side instrument Tine.

3.58 VERIFY Bag Fitter high differential pressure alarm PDAH-AY102WF-2 clears on graphics screen O6EvpAY1.v.

Test Engineer:

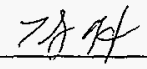

3.59 RECORD the conductivity reading indicated on the Clearview controller.

2. 3.59 .1 Tower fluid conductivity $1 \geq 0$ Micromhos Test. Engineer:

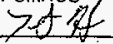

3.60 PLACE the Test Switch on the Clearview Controller in the TEST position.

3.61 VERIFY Evaporator Tower Bleed Valve EV-AY102EWT-1A2 OPENS.

Test Engineer:

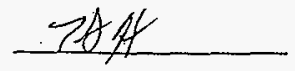




\section{PREOPERATI}

Revision No. 0
L TESTING, RECIRCULATION CONDEN acR COOLING WHC. SO- W03O- POTP- 00'

ATTACHMENT B

TANK AY102 RECIRC CONDENSER 1
HNF-SD-W030-TD-003,

REV. O, PAGE 57

3.62 PLACE the Test Switch on the Clearview Controller in the OPER position.

3.63 VERIFY Evaporator Tower BTeed VaTve EV-AY102EWT-1A2 CLOSES. Test Engineer:

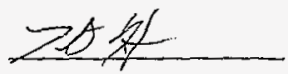

3.64 PLACE the Closed Loop Filtration System Recirc. Pump AY102-W-P-1 local control switch AY102WP-1 in OFF.

3.65 CLOSE the Closed Loop Filtration System inlet and outlet block valves HV-AY102W-1 and HV-AY102W-3.

3.66 If desired. THEN DRAIN the Closed Loop Filtration System as follows. OTHEPWISE continue at step 3.67:

3.66.1 REMOVE drain plug from Closed Loop Filtration System Recirc Pump casing.

3.66.2 DISCONNECT hoses on either side of bag filter.

3.66.3 REMOVE vent plug from top of vent filter.

3.66.4 WHEN system completes draining. THEN reinsta17 al7 plugs and connect hoses to bag filter.

3.67 PLACE Evaporator Tower AY102-EW-T-1 Spray Pump AY102-EW-SP-1 remote control switch HS-AY102-EWSP-1 in STOP.

3.68 VERIFY Evaporator Tower AY102-EW-T-1 Spray Pump AY102-EW-SP-1 STOPS.

Test Engineer:

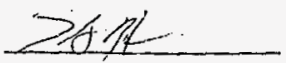

3.69 PLACE Evaporator Tower AY102-EW-T-I remote control switch HSAY102-EWT-1A1 in OFF.

3.70 VERIFY the following events occur:

3.70.1 AY102 Evaporator Tower Cooling Fan STOPS.

3.70.2 AY102 Evaporator Tower AY102-EW-T-1 Temperature Control Damper OPENS.

Test Engineer:

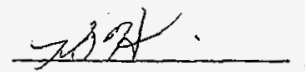

3.71 CLOSE Raw Water supply to the Evaporator Tower HV-AY102RW-1. 
PREOPERATIONAL TESTING, RECIRCULATION CONDENSER COOLING WHC-SD-W030-POTP-OC

Revision No. 0 ATTACHMENT B HNF-SD-W030-TD-003, REV. 0, PAGE 58 TANK AY102 RECIRC CONDENSER

3.72 If it is desired to drain the Evaporator Tower. THEN PERFORM the following steps. OTHERWISE continue at step 3.75 .

3.73 PLACE the Test Switch on the Clearview Controller in the TEST position to allow the Evaporator Tower to drain.

3.74 PLACE the Test Switch on the Clearview Controller in the OPER position.

3.75 VERIFY all test equipment installed to support this test has been removed.

Test Engineer:

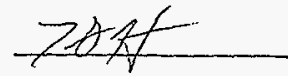


PREOPERAT, ...AL TESTING, RECIRCULATION CONDENSER COOLING WHC. SO $\cdot$ W030-POTP.003 PAGt

Revision No. $\Omega$ ATTACHMENT $B$

Effective Date REV. O, PAGE 59 2 RECIRC CONDENSER COOLING SYSTEM

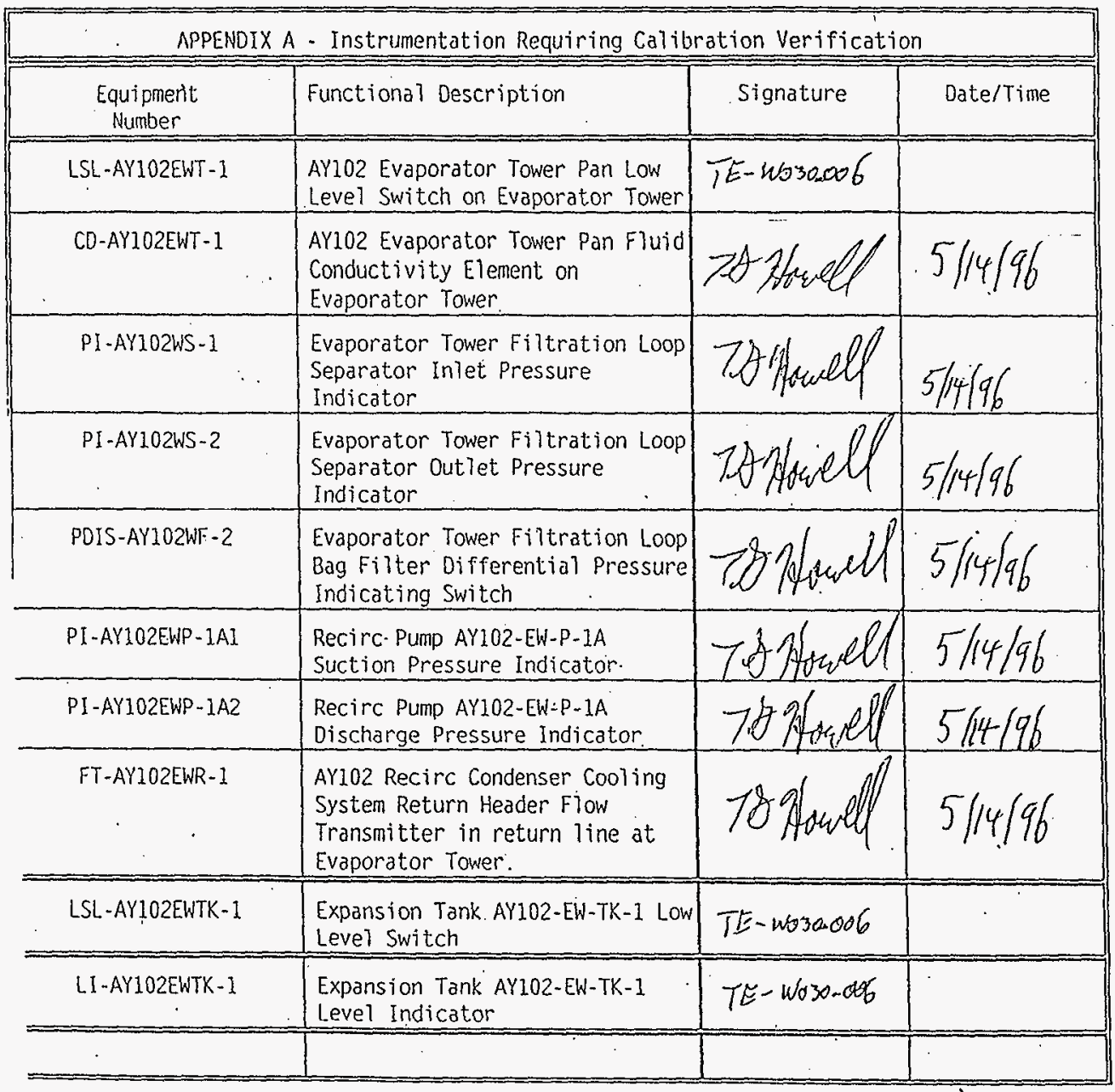


PREOPERATIONAL TESTING, RECIRCULATION CONDENSER COOLING

HNF-SD-W030-TD-003 WHC - SD - W030-P0TP-003

PAGE

Revision No. 0

ATTACHMENT B

Effective Date

TANK AY102 RECIRC CONDENSER COOLING SYSTEM

\begin{tabular}{|c|c|c|c|c|}
\hline $\begin{array}{l}\text { VALVE } \\
\text { NUMBER }\end{array}$ & VALVE NAME & $\begin{array}{l}\text { REQUIRED } \\
\text { POSITION }\end{array}$ & $\begin{array}{c}\text { INITIALS } \\
\left(I^{*}\right)\end{array}$ & $\begin{array}{l}\text { IV } \\
(2)\end{array}$ \\
\hline HV-AY102EWP-1AI & $\begin{array}{l}\text { Recirc Pump AY102-EW-P-1A } \\
\text { Suction Isolation Valve }\end{array}$ & OPEN & S. cak & $T . \angle W$ \\
\hline HV-AY102EWP-IA3 & $\begin{array}{l}\text { Recirc Pump AY102-EW-P-1A } \\
\text { Suction Pressure Gage Isolation }\end{array}$ & OPEN & $\cos$ & TKWN \\
\hline HV - AY102EWP-1A4 & $\begin{array}{l}\text { Recirc Pump AY102-EW-P-1A } \\
\text { Discharge Pressure Gage Isolation }\end{array}$ & OPEN & ). $\cos$ & TKaL \\
\hline HV-AY102EWP-1A5 & $\begin{array}{l}\text { Recirc Pump AY102-EW-P-1A } \\
\text { Discharge Isolation }\end{array}$ & CLOSED & D. cak & TKW \\
\hline HV-AY102EWS-1A2 & $\begin{array}{l}\text { Recirc Pump AY102-EW-P-1A } \\
\text { Discharge Header Isolation }\end{array}$ & OPEN & $\operatorname{con}$ & Then \\
\hline HV-AY102EWP-1B1 & $\begin{array}{l}\text { Recirc Pump AY102-EW-P-IB } \\
\text { Suction Isolation Valve }\end{array}$ & OPEN & D. cer & $T K \omega-$ \\
\hline HV-AY102EWP-1B3 & $\begin{array}{l}\text { Recirc Pump AY102-EW-P.1B } \\
\text { Suction Pressure Gage Isolation }\end{array}$ & OPEN" & 0. $\mathrm{cm}$ & JXW. \\
\hline HV-AY102EWP- 184 & $\begin{array}{l}\text { Recirc Pump AY102-EW-P-1B } \\
\text { Discharge Pressure Gage Isolaticn }\end{array}$ & OPEN & emp & $T K W$ \\
\hline HV-AY102EWP-1B5 & $\begin{array}{l}\text { Recirc Pump AY102-EW-P-1B } \\
\text { Discharge Isolation }\end{array}$ & CLOSED & in & TKW: \\
\hline HV-AY102-EWS-1AI & $\begin{array}{l}\text { Recirc Condenser Cooling System } \\
\text { Supply Header Drain Valve in } 241 \text {. } \\
\text { AY-402. valve box. }\end{array}$ & CLOSED & Bar & Trees \\
\hline HV-AY102-EWR-1AI & $\begin{array}{l}\text { Recirc Condenser Cooling System } \\
\text { Return Header Drain Valve 241-AY- } \\
402 \text { valve box. }\end{array}$ & CLOSED & & TKN- \\
\hline $\begin{array}{l}\text { HV-AY102-EWTK- } \\
\text { IA1 }\end{array}$ & $\begin{array}{l}\text { Expansion Tank Level Switch LSL. } \\
\text { AY102EWTK-1 Low Side Isolation }\end{array}$ & OPEN & Su ch & TKK \\
\hline $\begin{array}{l}\text { HV - AY102-EWTK- } \\
\text { IA2 } \\
\end{array}$ & $\begin{array}{l}\text { Expansion Tank Level Switch LSL- } \\
\text { AY102EWTK-1 High Side Isolation }\end{array}$ & OPEN & Eu. ant & TKKN \\
\hline HV-AY102-EWTK-2 & $\begin{array}{l}\text { Recirc Condenser Cooling System Air } \\
\text { Separator Drain Valve }\end{array}$ & CLOSED & $\operatorname{con}$ & TKR \\
\hline HV-AY102-EWT-1AI & Cooling Tower Pan Drain Valve & CLOSED & $\cos$ & TKN \\
\hline HV-AY102-RW-1 & $\begin{array}{l}\text { Cooling Tower Raw Water Isolation } \\
\text { at Evaporator Tower. }\end{array}$ & CLOSED & $\cos$ & TRe \\
\hline $\mathrm{HV}-\mathrm{AY} 102 \mathrm{~W}-1$ & $\begin{array}{l}\text { Closed Loop Filtration System Inlet } \\
\text { Block Valve }\end{array}$ & CLOSED & $\cos$ & THed \\
\hline
\end{tabular}




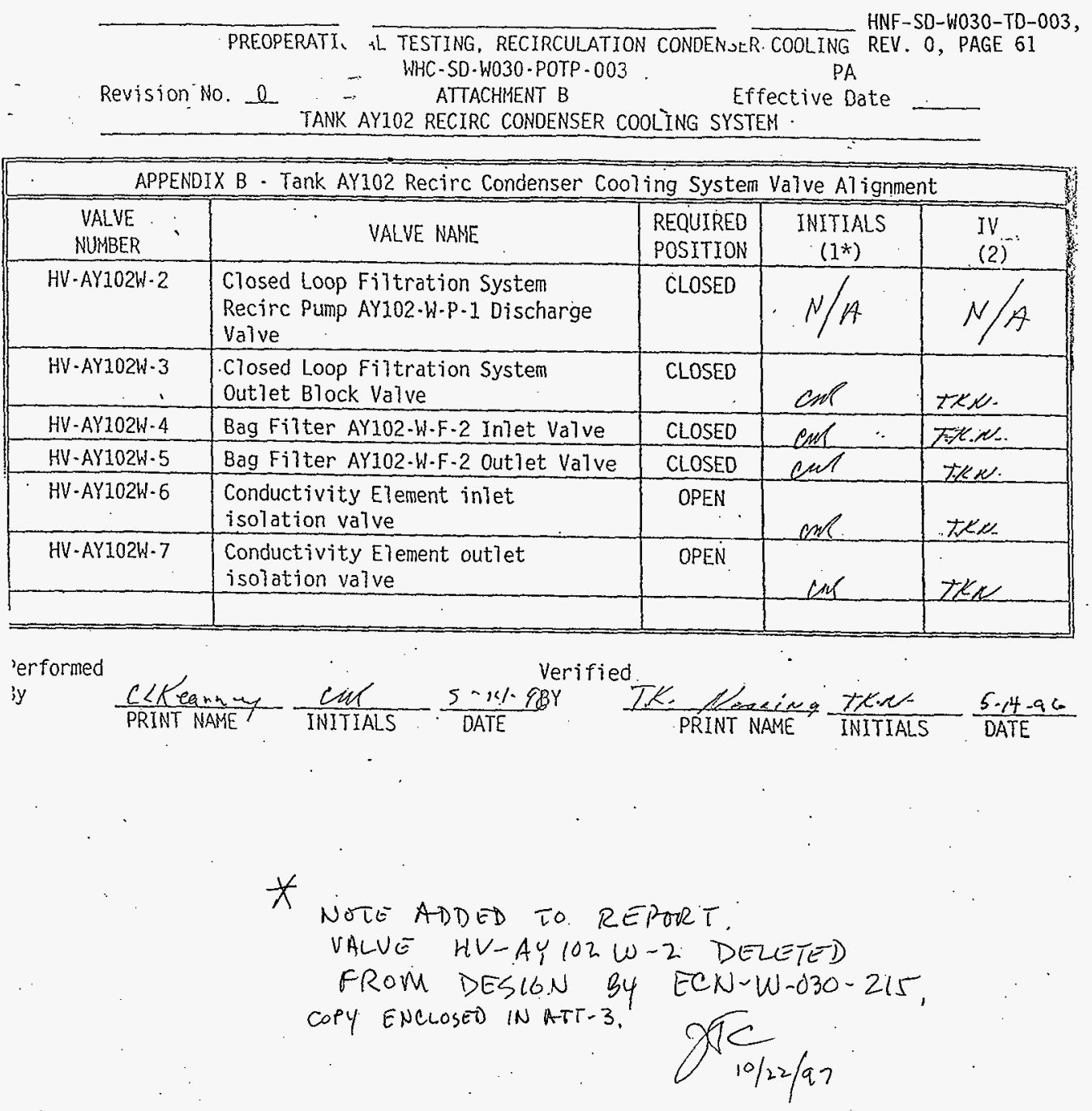




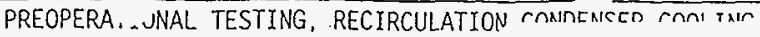

WHC.SD - W030-POTP-003

HNF-SD-W030-TD-003,

Revision No. 0

ATTACHMENT B

REV. 0, PAGE 62

TANK AY102 RECIRC CONDENSER CO

\begin{tabular}{|c|c|c|c|c|}
\hline $\begin{array}{l}\text { BREAKER , } \\
\text { NUMBER }\end{array}$ & BREAKER NAME AND LOCATION & $\begin{array}{l}\text { REQUIRED } \\
\text { POSITION }\end{array}$ & $\begin{array}{c}\text { INITIALS } \\
\left(1^{*}\right) \\
\end{array}$ & $\begin{array}{l}\text { IV } \\
\text { (2) }\end{array}$ \\
\hline $1 \mathrm{~B}$ & $\begin{array}{l}\text { Evaporator Tower AY102 Supply } \\
\text { Breaker in Distribution Panelboard } \\
\text { PP.1 Diesel Generator Building }\end{array}$ & ON & $\begin{array}{l}78) \not 1 \\
\text { cink }\end{array}$ & 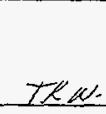 \\
\hline 2 HP SEPARATOR & $\begin{array}{l}\text { Evaporator Tower AY102 Separator } \\
\text { Recirc Pump Supply Breaker in Loca] } \\
\text { Panel AY102-EVAP.TWR }\end{array}$ & OFF & 78 & TKKH. \\
\hline $\begin{array}{l}\text { FLUID COOLER } \\
\text { CONTROL PANEL } \\
\text { AY102-EW-T-I }\end{array}$ & $\begin{array}{l}\text { Evaporator Tower AY102 Fan, Recirc } \\
\text { Pump, Pan Heater Supply Breaker in } \\
\text { Local Panel AY102-EVAP. TWR }\end{array}$ & OFF & $\begin{array}{l}7 \& 21 \\
\text { cen }\end{array}$ & TEx \\
\hline $\begin{array}{l}\text { RECIRC PUMP } \\
\text { AYI02:EW-P-1A }\end{array}$ & $\begin{array}{l}\text { AY102 Recirc Condenser Cooling } \\
\text { System Recirc Pump AY102-EW-P-1A in } \\
\text { Local Panel AY102-EVAP. TWR }\end{array}$ & OFF. & & THW. \\
\hline $\begin{array}{l}\text { RECIRC PUMP } \\
\text { AY102-EW-P-1B }\end{array}$ & $\begin{array}{l}\text { AY102 Recirc Condenser Cooling } \\
\text { System Recirc Pump AY102-EW-P-1B in } \\
\text { Local Pane1 AY102-EVAP-TWR }\end{array}$ & OFF & $\begin{array}{l}7 A g \\
\cos \end{array}$ & $\pi K \mu$ \\
\hline PANEL PP-7 & $\begin{array}{l}\text { Evaporator Tower Panelboard PP.7 } \\
\text { Supply Breaker in Local Panel } \\
\text { AY101-EVAP.TWR }\end{array}$ & ON & $7 \& q$ & $T K N$ \\
\hline $\begin{array}{l}\text { Disconnect } \\
\text { switch }\end{array}$ & $\begin{array}{l}\text { Evaporator Tower AY102 Separator } \\
\text { Recirc Pump local disconnect switch } \\
\text { at pump. }\end{array}$ & OFF & 78 & TKw \\
\hline & & & & \\
\hline & & & & \\
\hline & & & & \\
\hline & & & & \\
\hline & & & & \\
\hline+ & & & & \\
\hline & & & & \\
\hline
\end{tabular}

'formed

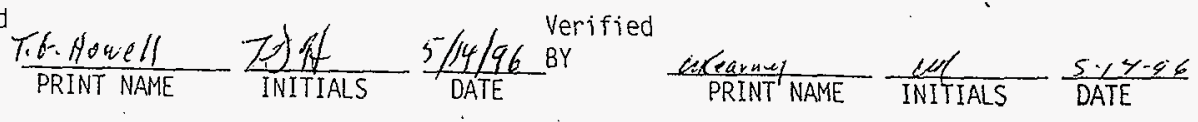


PREOPERAT IAL TESTING, RECIRCULATION CONDENSER COOLING REV. 0, PAGE 63 WHC-SD-W030-POTP-003

Revision No. 0

ATTACHMENT B

PAI

Effective Date

TANK AYI02 RECIRC CONDENSER COOLING SYSTEM

APPENDIX $0-1$

OPERATION DATA SHEET

Equipment Name: 'IANK AY102 RECIRC CONDENSER COOLING SYSTEM RECIRC PUMP

EQUIPMENT I.D. NO. : AYIO2-EW-P-IA

NAMEPLATE DATA:

See Appendix F. ge

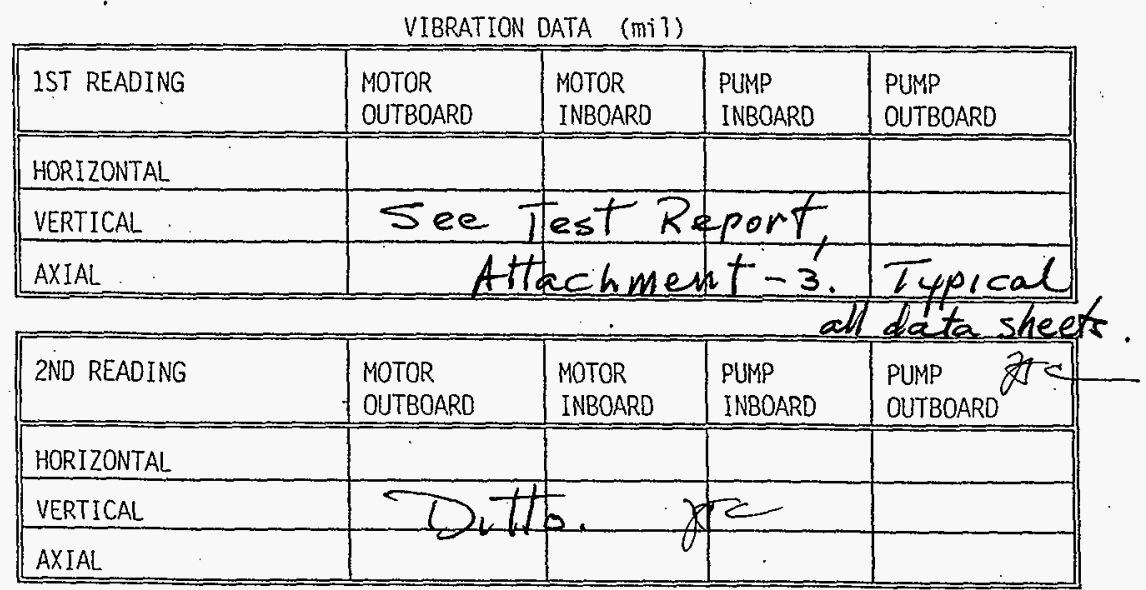

\begin{tabular}{|l|l|l|l|l|}
\hline $\begin{array}{l}\text { PUMP BEARING } \\
\text { TEMPERATURES }\end{array}$ & $\begin{array}{l}\text { MOTOR } \\
\text { OUTBOARD }\end{array}$ & $\begin{array}{l}\text { MOTOR } \\
\text { INBOARD }\end{array}$ & $\begin{array}{l}\text { PUMP } \\
\text { OUTBOARD }\end{array}$ & PUMP INBOARD \\
\cline { 2 - 4 } & & & & \\
\hline
\end{tabular}

IARKS:

Performed by :

Date

Verified by :

Date 
PREOPERAT .. NAL TESTING, RECIRCULATION CONDENSER COOLING WHC-SO-WO3O-POTP - OC

Revision No. 0

ATTACHMENT $B$

HNF-SD-W030-TD-003,

TANK AY102 RECIRC CONDENSER

REV. 0, PAGE 64

APPENDIX F

TEST LOG

HiEST TIIL:

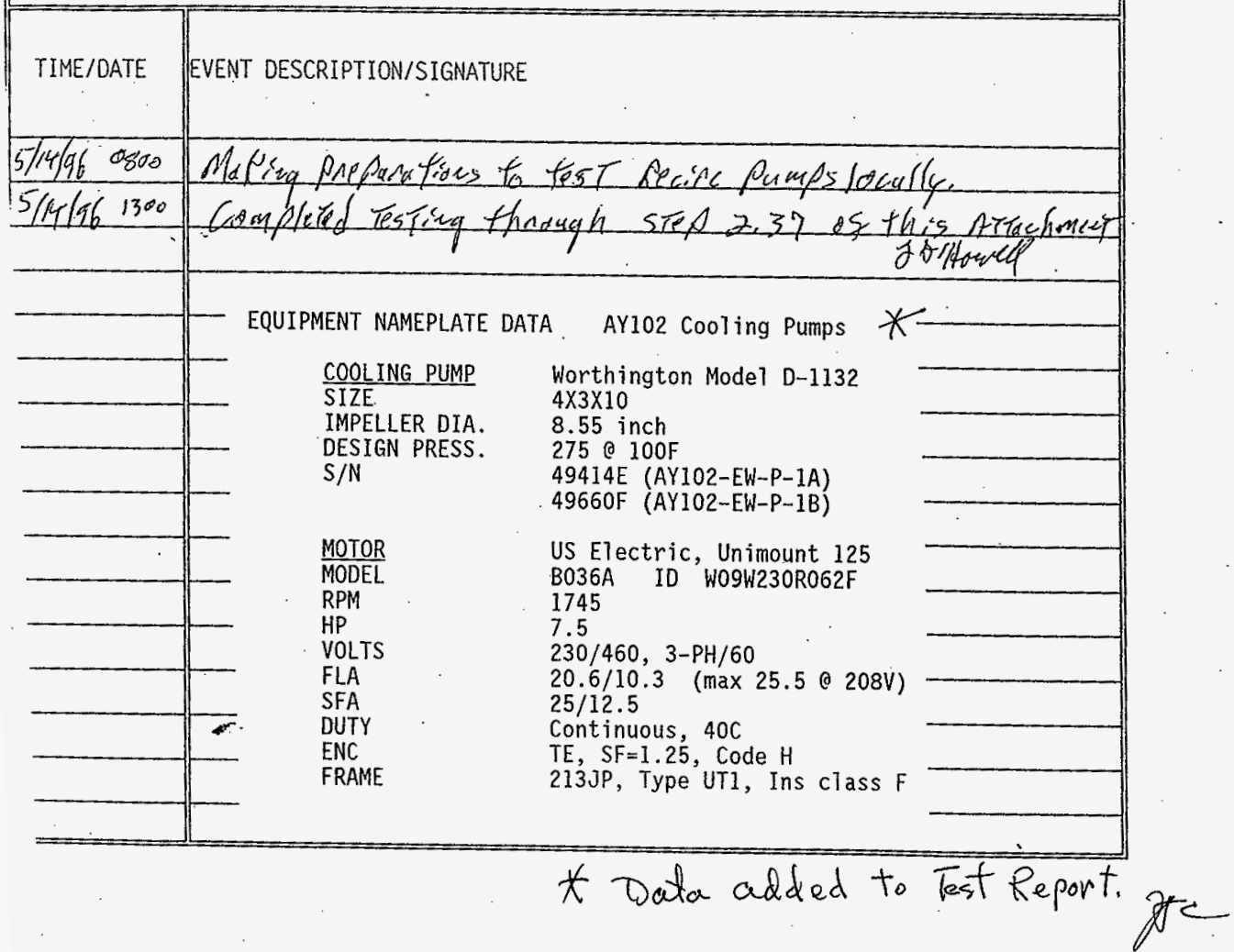


PREOPEF .ONAL TESTING, RECIRCULATION CON. _NSER COOLING WHC-SD-W030-POTP-003

Revision No. 0

ATACHENT

TANK AZTO2 RECIRC CONDENSER CC

HNF-SD-W030-TD-003, A.Ylo2

REV. O, PAGE 65 APPENDIX $F$

TEST LOG TEST NUHEER:

TESTI lOG

PAGE NLMBSR:

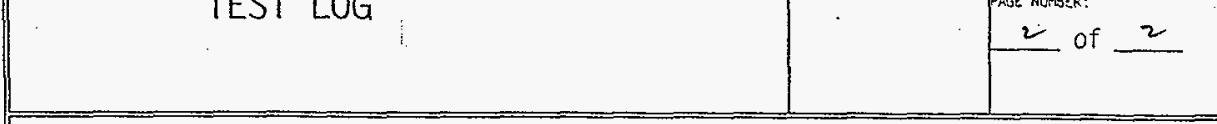

TEST TITLE:

TIME/DATE

EVENT DESCRIPTION/SIGNATURE

EQUIPMENT NAMEPLATE DATA AY102 COOLING TOWER

TOWER Baltimore Aircoil (BAC)

MODEL F1-742-K

S/N $\quad 94200708$

BELT B73

PUMPS SPRAY PUMP

MODEL $\quad$ BAC \#300211

FILTER PUMP

SIZE $\quad 150 \mathrm{GPM}$, TDH $12 \mathrm{ft}$

IMP. DIA, 3.0 inch

$S / N \quad 0 . J M 022394 I$

TYPE

MOTOR

$S / N$

MODEL

RPM

HP

VOLTS

FLA

SFA

DUTY

ENC

FRAME

General Electric

ZHL010910

5K38PN48 (cat K221)

3450

*

Grundfos B9414

85 GPM, TDH $61 \mathrm{ft}$ O3500RPM

$-$

EP150 2020 U-B-A-BBVP

Baldor Cat. \#VM3555-NP

F394

Spec. \#35A13-672F5

3450

1

2

208-230/460, 3-PH/60

208-230/460

3.3-3.2/1.6

4.7-4.4/2.2

Continuous, $40 \mathrm{C}$

$S F=1.4$, Code $M$,

$5.7-5.4 / 2.7$

Continuous, $40 \mathrm{C}$

Ins. class $B$

$T E, S F=1.15$, Code $H$

Ins. Class $B$

$56 \mathrm{~J}$

$56 \mathrm{C}$

* Data added to Test Report. If 
PREOPEI IONAL TESTING, RECIRCULATION CONDENSER COOLING REV. 0, PAGE 66 WHC-SD-W030-POTP-003

Revision No. Q

ATTACHMENT B

Effective Date

TANK AY102 RECIRC CONDENSER COOLING SYSTEM

APPENDIX G

TEST EXCEPTION REPORT

\begin{tabular}{|c|c|c|}
\hline TEST PRQCEOUSE WO. \& SECTION: & TEST NAME: & T.E. MLMER: \\
\hline OESCRIPTION OF PROBLEM: & & \\
\hline
\end{tabular}

ORIGINATOR:

ORG:

DATE:

O15POSITION:
IHPACT OA TESTIHG: O HOLO FOR RESOLUTION D CONTINUE

PIC DATE

ISPOSITION AND RETEST REQUIREMENTS BY:

DATE

E CONCURRENCE WITH DISPOSITION (If required):

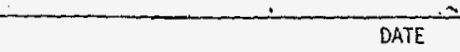

DISPOSITION ACTIONS COMPLETE:

Verified

By: DATE

REYET CONPLTE:

PIC

DATE 


\begin{tabular}{|c|c|c|}
\hline$P$ & MTIONAL TESTING, RECIRCUL & R COOLING \\
\hline Revision No. & $\begin{array}{l}\text { WHC.SD-W030-POTP - } \\
\text { ATTACHMENT C } \\
\text { TANK AZ101 RECIRC CONDENSE }\end{array}$ & $\begin{array}{l}\text { HNF-SD-W030-TD-003, } \\
\text { REV. O, PAGE } 67\end{array}$ \\
\hline
\end{tabular}

\subsection{INITIAL CONDITIONS}

1.1 VERIFY all. system instrumentation in Appendix $A$ is calibrated and has a current calibration tag affixed to each instrument and that all loop calibrations are complete.

Test Engineer:

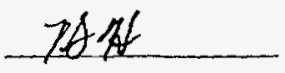

1.2 VERIFY the system is aligned for preoperational testing in accordance with Appendix $B$.

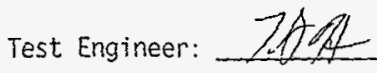

1.3 VERIFY system electrical circuit breakers are aligned in accordance with Appendix $C$.

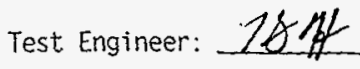

1.4 VERIFY expansion tank jevel is at midlevel as indicated on LIAZ101EWTK-1.

Test Engineer:

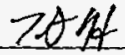

1.5 All personnel who will be involved with this procedure have provided the required signature verification information in Appendix E.

Test Engineer:

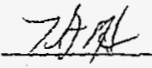

1.6 The test engineer has verified. by review of the $\mathrm{tag}$ log and a walkdown of the systems being tested, that all components within and including the test boundary have been "blue" tagged.

Test Engineer:

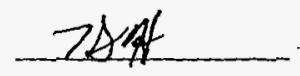

2.0 Tank AZ101 Glycol Recirc System Testing

2.1 VERIFY Recirc PunP A.Z101-EW-P-1A local control switch HS-AZ101EWP-

- 1 A 1 in the STOP position.

Test Engineer:

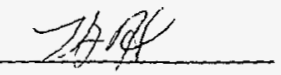

2.2 CLOSE local breaker for Recirc Pump AZ101-EW-P-1A. 


PREOPERATIUNAL TESTING, RECIRCULATION CONDENSFR COOLING
WHC-SO-W030-POTP-00
ATTACHMENT C

2.3 RECORD the following data for Recirc Pump AZ101-EW-P-1A:

Expansion Tark Level (Local)

Suction Pressure(Local)

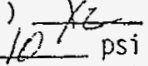

Run Time (graphics screen 06EvpAYl.v) 5 hrs

Test Erigineer:

hrs

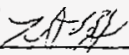

2.4. :ATTEMPT to start Recirc Pump AZ101-EW-P-1A from MCS graphics screen 06EvpAY1.V.

2.5 VER1FY Recirc Pump AZ101-EW-P-1A does not start.

Test Engineer:

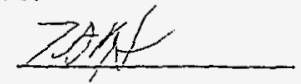

2.6 BUMP Recirc Pump AZ101-EW-P-1A by placing control switch HSA2101EWP-1A1 in START and then back to STOP.

2.7 VERIFY rotation of the Recirc Pump AZ101-EW-P.1A is in the direction indicated by the arrow on the pump.

$\therefore \therefore$

Test Engineer:

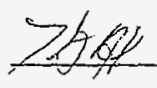

2.8 PLACE Recirc Pump AZ101-EW-P-1A local contro? switch HS-AZ101EWPIAI in the START position.

2.9 VERIFY Recirc Pump AZ101-EW-P-1A starts.

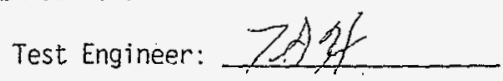

2.10 RECORD the following data for Recirc Pump AZ101-EW-P-1A:

$2.10 .1 \quad$ Motor Current (graphics screen 06EvpAY1.v) 13

$\therefore$ Amps

2.10 .2 Motor voltage (TEST INST.) 499 Volts

2.10 .3 Suction Pressure. (LOCAL) $\frac{10}{10}$ psi

ar. 2.10 .4 Discharge Pressure (LOCAL)

Test Engineer:

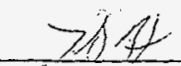

2.I1 SLOWLY OPEN Recirc pump AZ101-EW-P-IA discharge Valve HV-AZ101EWP$1 A 5$ and establish a system flowrate of $2327 \mathrm{gpm}$ as indicated on FI-AZ101EWR-1 on graphics screen 06EvpAY1.v. 
PREOPER, IONAL TESTING, RECIRCULATION COM

WHC-SD-W030-POTP-00

Revision No. 0

ATTACHMENT $C$

HNF-SD-W030-TD-003,

REV. 0, PAGE 69

TANK AZ101 RECIRC CONDENSER :

2.12 VERIFY low flow alarm FAL-AZ101WR-1 clears graphics screen 06EvpAY1.v.

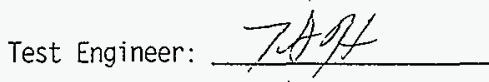

2.13 RECORD the following data for Recirc Pump AZ101-EW-P-1A:

2.13.1 Motor Current (graphics screen 06EvpAY1.v) 19,5 Amps

2.13.2 Motor Voltage (TEST INST.) 4999 Volts

2.13.3 Suction Pressure (LOCAL) - -5 psi

2.13.4 Discharge Pressure (LOCAL) EL psi

2.13.5 System Flow (graphics screen 06EvpAY1.v) $328 \mathrm{gpm}$

2.13.6 . Expansion Tank Leve] (Local) y/z

Test Engineer:

2.14 THROTTLE CLOSED valve HV-AZ101EWP-1A2 unti] system flow starts to decrease as indicated on FI-AZ101EWR-1 graphics screen 06EvpAY1.V.

2.15 FULLY OPEN Recirc Pump AZ101-EW-P-1A discharge valve HV-AZ101EWP1 A5.

2.16 ADJUST system flow to $\geq 327 \mathrm{gpm}$ using HV-AZ101EWP-IA2

2.17 VERIFY Recirc Pump AZ101-EW-P-1B. local control switch HS-AZ101EWP$1 B I$ in the STOP position.

Test Engineer:

2.18 CLOSE local breaker for Recirc PUMP AZ101-EW-P-1B.

2.19 RECORD the following data for Recirc Pump AZ101-EW-P-1B:

Suction Pressure(Local)

Run Time (graphics screen 06EvpAY1.v)

Test Engineer: hrs.

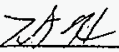

2.20 BUMP Recirc Pump AZ101-EW-P-1B by placing control switch HSAZ101EWP-1BI in START and then back to STOP.

2.21 VERIFY rotation of the Recirc Pump AZ101-EW-P-1B is in the direction indicated by the arrow on the pump.

Test Engineer: 


\begin{tabular}{ccc}
\hline PREOPEK & $\begin{array}{c}\text { ONAL TESTING, RECIRCULATION COR . NSER COOLING } \\
\text { WHC-SD-WO30-POTP-0 }\end{array}$ & HNF-SD-WO30-TD-003, \\
ATTACHMENT C & REV. 0, PAGE 70 \\
Revision No. $\&$ & TANK AZ101 RECIRC. CONDENSER &
\end{tabular}

2.22 ATTEMPT to start Recirc Pump AZ101-EW-P-1B from MCS graphics screen 06EvpAY1.v.

2.23 VERIFY Recirc Pump AZ101-EW-P-IB does not start.

Test Engineer:

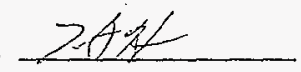

2.24 PLACE Recirc Pump AZ101-EW-P-1B local control switch HS-AZ101EWP$1 B 1$ in the START position.

2.25 VERIFY Recirc Pump AZ101-EW-P-1B starts.

Test Engineer:

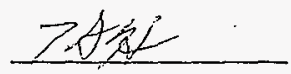

2.26 RECORD the following data for Recirc Pump AZ101-EW-P-1B:

2.26.1 Motor Current (gräphics screen 06EvpAYI.v) $\perp 3$ Amps

2.26 .2

Motor Voltage (TEST INST.

2.26 .3

Suction Pressure (LOCAL)

Discharge Pressure (LOCAL)

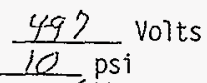

2.26 .4

Test Engineer:

2.27 SLOWLY OPEN Recirc pump AZ101-EW-P-IB discharge valve HV-AZ101EWP185:

2.28 ATTEMPT to stop Recirc Pump AZ101-EW-P-1A from MCS graphics screen 06EvpAY1.v.

2.29 VERIFY that Recirc Pump AZ101-EW-P-1A does not STOP.

Test Engineer:

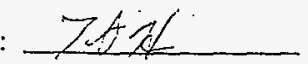

2.30 PLACE Recirc Pump AZ101-EW-P-1A local control switch HS-AZ101EWPIA1 in the STOP position.

2.31 VERIFY that Recirc Pump AZ101-EW-P-1A STOPS.

Test Engineer:

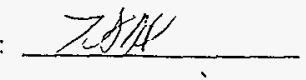

2.32 ADJUST system flow, if required, to z192 gpm using HV-AZI01EWP$1 \mathrm{~A} 2$. 
PREOPE:

2.33 RECORD the following data for Recirc Pump AZ101-EW-P-IB:

2.33 .1

Motor Current (graphics screen 06EvpAY1.v) $\geq L$ Amps

2.33 .2

Motor Voltage (TEST INST.) $\quad 499$ volts

2.33 .3

Suction Pressure (LOCAL)

2.33 .4

Discharge Pressure (LOCAL) 5 psi

2.33 .5

2.33 .6

System Flow (graphics. screen 06EvpAY1.v) 332 gpm

Expansion Tank Level (Local)

Test Engineer:

2

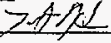

2.34 THROTTLE HV-AZ101EWP-1A2 to decrease flow to $<295 \mathrm{gpm}$.

2.35 VERIFY system low flow alarm. FAL-AZ101EWR-1 annunciates on graphics screen 06EvpAYl.v.

Test Engineer:

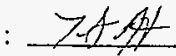

2.36 THROTTLE HV-AZ101EWP-1A2 to increase flow to $\geq 327 \mathrm{gpm}$.

2.37 VERIFY system low flow alarm, FAL-AZ101EWR-1 clears on graphics screen 06EvpAY1.v.

Test Engineer:

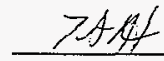

2.38 PLACE Recirc Pump AZ101-EW-P-1A local control switch HS-AZ101EWPIAI in the REMOTE position.

2.39 START Recirc Pump AZ101-EW-P-1A from graphics screen 06EvpAY1.V

2.40 VERIFY Recirc Pump AZ101-EW-P-1A starts. Test Engineer:

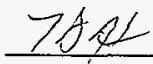

2.41 PLACE Recirc Pump AZ101-EW-P-1B local control switch HS-AZ101EWP$1 B 1$ in the STOP pOsition.

2.42 VERIFY Recirc Pump AZ101-EW-P-1B stops.

Test Engineer:

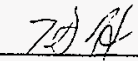

2.43 PLACE Recirc Pump AZ101-EW-P-IB local control switch HS-AZ101EWP$1 B 1$ in the REMOTE position.

2.44 PLACE Recirc Pump AZ101-EW-P-1B in Standby on graphics screen 06EvpAY1.V. 


\begin{tabular}{|c|c|c|}
\hline PREOPERA; & NAL TESTING, RECIRCULATION CONDL & . ER COOLING \\
\hline Revision No. $\underline{0}$ & $\begin{array}{c}\text { WHC-SD-W030-POTP- } \\
\text { ATTACHMENT C } \\
\text { AZ101 RECIRC CONDENSEF }\end{array}$ & $\begin{array}{l}\text { HNF-SD-WO30-TD-003 } \\
\text { REV. 0, PAGE } 72\end{array}$ \\
\hline
\end{tabular}
TANK AZ101 RECIRC CONDENSEI

2.45 OPEN local breaker switch for Recirc Pump AZ101-EW-P-1A.

2.46 VERIFY RecirC Pump AZ101-EW-P-1B auto starts. Test Engineer:

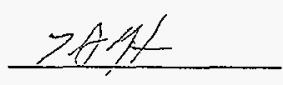

2.47 PLACE Recirc Pump AZ101-EW-P-1A remote control switch HS-AZ101EWP$1 A$ in STOP position on graphics screen 06EvpAY1.V.

2.48 CLOSE local breaker for Recirc Pump AZ101-EW-P-1A.

2.49 PLACE Recirc Pump AZ101-EW-P-1A in the Standby on graphics screen 06EvPAY1.V.

2.50 OPEN local breaker for Recirc Pump AZ101-EW-P-1B.

2.51 VERIFY Recirc Pump AZ101-EW-P-1A auto starts. Test Engineer:

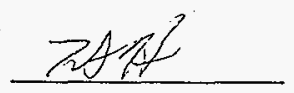

2.52 PLACE Recirc Pump AZ101-EW-P-1B remote control switch HS-AZ101EWP$1 B$ in STOP position on graphics screen 06EvpAYl.v.

2.53 CLOSE local preaker for Recirc PUmp AZ101-EW-P-1B.

2.54 ALLOW Recirc Pump AZ101-EW-P-IA to operate for one hour for collection of vibration data and bearing data.

2.55 RECORD vibration data and bearing temperatures on Appendix D-1.

Test Engineer:

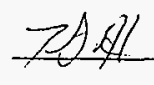

2.56 START Recirc Pump AZ101-EW-P-1B remotely from LCU4-17.

2.57 STOP Recirc Pump AZ101-EW-P-IA remotely from LCU4-16.

2.58 ALLOW Recirc Pump A2101-EW-P-1B to operate for one hour for collection of vibration data and bearing data.

2.59 RECORD vibration data and bearing temperatures on Appendix $0-2$. Test Engineer: Zifl 
PREOPER ¿ONAL TESTING, RECIRCULATION COI. JSER COOLING WHC-SD-W03O-POTP-OC

Revision No. 2

ATTACHMENT C

TANK AZ101 RECIRC CONDENSER
HNF-SD-W030-TD-003,

REV. O, PAGE 73

2.60 SLOWLY CLOSE Expansion Tank Level Switch LSL-AZ101EWTK- 1 Low Side Isolation Valve HV-AZ101EWTK-1A1.

2.61 VERIFY Expansion Tank Low Level Alarm LAL-AZ101EWTK-1 annunciates on graphics screen 06EvpAY1.v.

Test Engineer:

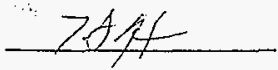

2.62 OPEN Expansion Tank Level Switch LSL-AZ101EWTK- 1 Low Side ISolation Valve HV-AZ101EWTK-1A1.

2.63 VERIFY Expansion Tank LOW Level Alarm LAL-AZ101EWTK-1 clears on graphics screen 06EvpAY1.v.

Test Engineer:

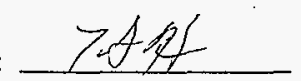

2.64 PLACE Low Flow Shutdown switches HS-AZ101EWP-1AB and HS-AZ101EWPIBB in the OVERRIDE position on graphics screen 06EvpAYI.v.

-2.65 PLACE Recirc Pump AZ101-EW-P-1A in STANDBY on graphics screen 06EvPAY1.V.

2.66 THROTTLE HV-AZ101EWP-1A2 to decrease flow to $<100 \mathrm{gpm}$.

2.67. VERIFY the Recirc Pump AZ101-EW-P-IB does not STOP on low flow.

Test Engineer:

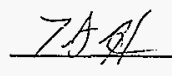

2.68 PLACE Low Flow Shutdown Override switch HS-AZ101EWP-1BB in the NORMAL position from the LOI.

2.69 VERIFY the RecirC Pump AZ101-EW-P-1B STOPS on low flow.

Test Engineer:

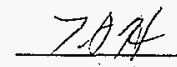

2.70 VERIFY Recirc Pump AZ101-EW-P-1A starts.

Test Engineer:

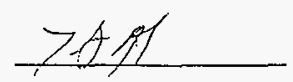

2.71 PLACE LOW Flow Shutdown Override switch HS-AZ101EWP-1AB in the NORMAL position on graphics screen 06EvpAYI.V.

2.72 VERIFY the Recirc Pump AZ101-EW-P-1A STOPS on low flow.

Test Engineer:

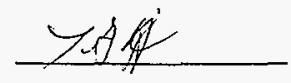




\begin{tabular}{|c|c|c|}
\hline \multicolumn{3}{|c|}{ PREOPERAT, UIAL TESTING, RECIRCULATION CONDE, ,UER COOLING } \\
\hline Revision No. 0 & $\begin{array}{l}\text { WHC - SD-W030-POTP-C } \\
\text { ATTACHMENT C }\end{array}$ & $\begin{array}{l}\text { HNF-SD-W030-TD-003, } \\
\text { REV 0, PAGE } 74\end{array}$ \\
\hline
\end{tabular}

2.73 PLACE Low Flow Shutdown Override switch HS-AZ101EWP-1AB in the OVERRIDE position on graphics screen 06EvpAY1.V.

2.74 RESET Recirc Pump AZ101-EW-P-1A by placing remote control switch HS-AZ101EWP-1A in STOP position on graphics screen 06EVPAYI.V.

2.75 PLACE RecirC Pump AZ101-EW-P-1A remote control switch HS-AZ101EWPIA in START position on graphics screen 06EvpAY1.v.

2.76 VERIFY that Recirc Pump AZ101-EW-P-1A starts.

Test Engineer:

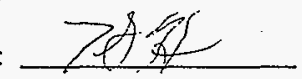

2.77 THROTTLE HV-AZ101EWP-IA2 to increase system flow to $2327 \mathrm{gpm}$.

2.78 PLACE Low Flow Shutdown Override switch HS-AZ101EWP-1AB in the NORMAL position on graphics screen 06EvpAY1.V.

\subsection{Tank AZ101 Evaporator Tower Testing}

3.1 REMOVE manway covers on the cooling tower.

3.2 OPEN HV-AZ101-RW-1 Cooling Tower Raw Water Isolation valve at Evaporator Tower.

3.3 VERIFY cooling tower fill via makeup valve EV-AZ101EWT-1Al.

Test Engineer:

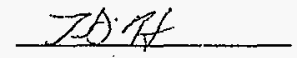

3.4 INSTALL manway covers when cooling tower fill is complete.

3.5. VERIFY the following local switch positions:

- Evaporator Tower AZ101-EW-T-1 Spray Pump AZ101-EW-SP-1 loca1 control switch HS-AZ101-EWSP-1A in OFF.

- Evaporator Tower AZ101-EW-T-1 Fan local control switch HSAZ101EWT-1A1 in OFF.

- Evaporator Tower AZ101-EW-T-1 Pan Heater local control switch HS-AZ101EWT-1A 2in OFF.

Test Engineer:

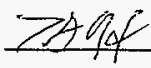

3.6 CLOSE the local supply breaker for the spray pump. cooling fan. and pan heater in the Evaporator Tower local distribution panel. 
PREi

Revision No. 0
.ZATIONAL TESTING, RECIRCULATION

WHC-SD-WO3O-POTP

ATTACHMENT C

TANK AZ101 RECIRC CONDENS
HNF-SD-W030-TD-003, REV. 0, PAGE 75

3.7 REMOVE splash guard from top of Spray Pump AZ101-EW-SP-1 motor to enable viewing of the motor shaft.

3.8 BUMP Spray Pump AZ101-EW-SP-1 by placing control switch HS-AZ101EWSP-1A in START and then back to OFF.

3.9 VERIFY rotation of the Spray Pump AZ101-EW-SP-1 is in the direction indicated by the arrow on the pump.

Test Engineer:

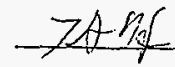

3.10 REINSTALL splash guard on Spray Pump AZ101-EW-SP-1 motor.

3.11 PLACE Evaporator Tower AZ101-EW-T-1 Spray Pump AZ101-EW-SP-1 locaT control switch HS-AZ101-EWSP-1A in START.

3.12 VERIFY Spray Pump AZ101-EW-SP-1 starts.

Test Engineer:

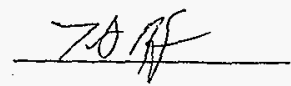

3.13 RECORD the following data for Spray Pump AZ101-EW-SP-1:

3.13.1 Motor Current (TEST INST.)

3.13 .2

Motor Voltage (TEST INST.)

1.7 Amps

3.13 .3

Spray Flow (TEST INST.)

Test Engineer:

gpm

3.14 ALLOW Spray Pump AZ101-EW-SP-1 to operate for one hour for collection of vibration data and bearing data.

3.15 RECORD vibration data and bearing temperatures on Appendix D-3.

Test Engineer:

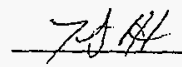

3.16 PLACE Evaporator Tower AZ101-EW-T-1 Spray Pump AZ101-EW-SP-I remote control switch HS-AZ101-EWSP-1 in STOP on graphics screen 06EvPAYI.V.

3.17 VERIFY Spray Pump AZ101-EW-SP-1 does NOT STOP. Test Engineer:

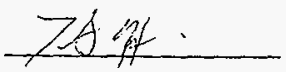

3.18 PLACE Evaporator Tower AZ101-EW-T-1 Temperature Control Damper local control Switch HS-AZ101-EWT-1A3 in OPEN. 
PREOPER .ONAL TESTING, RECIRCULATION CON NNSER COOLING WHC-SD-WO30-POTP-
Revision No. 0
ATTACHMENT C
TANK AZ101 RECIRC CONDENSE
HNF-SD-W030-TD-003,
REV. 0, PAGE 76

3.19 VERIFY Evaporator Tower AZ101-EW-T-1 Temperature Control Damper OPENS.

Test Engineer:

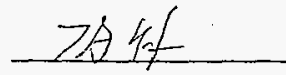

3.20 PLACE Evaporator Tower AZ101-EW-T-1 Cooling Fan local control switch HS-AZ101-EWT-1A1 in START.

3.21 VERIFY Evaporator Tower AZ101-EW-T-1 Cooling Fan starts.

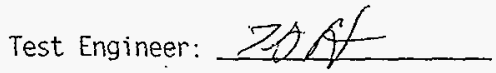

3.22 RECORD the following data for Evaporator Tower AZ101-EW-T-1 Cooling Fan:

3.22.1 Motor Current (TEST INST.) $1 \$, 2$ Amps

3.22.2 Motor Voltage (TEST INST.) 485 Volts

Test Engineer: ¿At?

3.23 ALLOW Evaporator Tower A2101-EW-T-1 Cooling Fan to operate for one hour for collection of vibration data and bearing data.

3.24 RECORD vibration data and bearing temperatures on Appendix D-4.

Test Engineer:

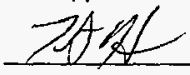

3.25 PLACE Evaporator Tower AZ101-EW-T-1 Cooling Fan remote control switch HS-AZ101-EWT-1 in OFF on graphics screen 06EvpAYI.v.

3.26 VERIFY Evaporator Tower AZ101-EW-T-I Cooling Fan does NOT STOP..

Test Engineer:

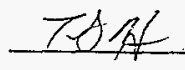

3.27 PLACE Evaporator Tower AZ101-EW-T-I Pan Heater local control switch HS-AZ101-EWT-1A2 in ON.

3.28 VERIFY Evaporator Tower AZ101-EW-T-1 Pan Heater remains deenergized by checking the heater contactor is OPEN.

3.29 SIMULATE a low Evaporator Tower Pan level.

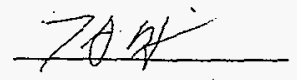


PREOPEK IONAL TESTING, RECIRCULATION CO. NSER COOLING WHC-SD-WO3O-POTP-OC

Revision No. 0

ATTACHMENT C

TANK AZ101 RECIRC CONDENSER

HNF-SD-W030-TD-003,

REV. 0, PAGE 77

3.30 VERIFY the following actions occur:

3.30.1 Evaporator Tower Spray Pump AZ101-EW-SP-1 stops.

3.30.2 Evaporator Tower Cooling Fan stops.

3.30.3 Evaporator Tower Makeup Valve EV-AZ101EWT-1Al gpens.

Test Engineer:

3.31 RECORD makeup flow at FI-AZRW-1 (graphics screen 05water.v): 23 gpm.

Test Engineer:

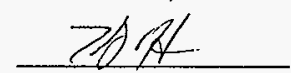

3.32 RESTORE the Evaporator Tower Pan level to normal.

3.33 VERIFY Evaporator Tower Pan Heater energizes by checking the heater contactor is CLOSED.

Test Engineer:

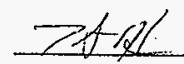

3.34 PLACE Evaporator Tower AZ101-EW-T-1 Temperature Control Damper local control Switch HS-AZ101-EWT-1A3 in AUTO.

3.35 PLACE Evaporator Tower AZ101-EW-T-1 Spray Pump AZ101-EW-SP-1 local control switch HS-AZ101-EWSP-1A in REMOTE.

3.36 PLACE Evaporator Tower AZ101-EW-T-1 Cooling Fan local control switch HS-AZ101-EWT-1A1 in REMOTE.

3.37 PLACE Evaporator Tower AZ101-EW-T-1 Spray Pump AZ101-EW-SP-1 remote control switch HS-AZ101-EWSP-1 in START on graphics screen 06EvpAY1.v.

3.38 VERIFY Evaporator Tower AZ101-EW-T-1 Spray Pump AZ101-EW-SP-1 starts.

Test Engineer: ZA) //4

3.39 VERIFY Evaporator Tower Pan Heater deenergizes by checking the heater contactor' is OPEN.

Test Engineer:

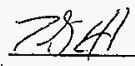

3.40 PLACE Evaporator Tower AZ101-EW-T-1 remote control switch HSAZ101-EWT-1A1 in ON on graphics screen O6EvpAY1.v. 
PREOPERATI . .AL TESTING, RECIRCULATION CONDE I. R COOLING WHC-SD-WO30-POTP-00:

Revision No.

ATTACHMENT C

HNF-SD-W030-TD-003,

TANK AZ101 RECIRC CONDENSER $C$

REV. 0, PAGE 78

3.41 VERIFY the following events occur:

3.41.1 AZ101 Evaporator Tower Cooling Fan starts.

3.41.2 AZ101 Evaporator Tower AZ101-EW-T-1 Temperature

Control Damper modulates to maintain glýcol

temperature at setpoint $100^{\circ} \mathrm{F}$ as indicated on graphics screen06EvpAYl.v.

Test Engineer:

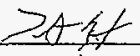

3.42 OPEN Closed Loop Filtration System inlet and outlet block vaives HV-AZ101W-1 and HV-AZ101W-3.

3.43 BUMP the Closed Loop Filtration System Recirc Pump AZ101-W-P-1 by placing control switch HS-AZ101WP-1 in HAND and then back to OFF.

3.44 VERIFY rotation of the Closed Loop Filtration System Recirc Pump AZ101-W-P-1 is in the direction indicated by the arrow on the pump.

Test Engineer:

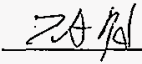

3.45 PLACE the Closed Loop Filtration System Recirc Pump AZ101-W-P-1 local control switch AZ101WP-1 in HAND.

3.46 VERIFY Closed Loop Filtration Recirc Pump AZ101-W-P-I starts.

Test Engineer:

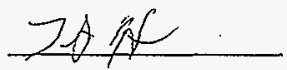

3.47 SLOWLY OPEN Closed Loop Filtration System Recirc Pump AZ101-W-P-I discharge valve HV-AZ101W-2 to establish full system flow.

3.48 RECORD the following data for Closed Loop Filtration Recirc Pump AZ101-W-P-1:

3.48 .1

3.48 .2

3.48 .3

3.48 .4

3.48 .5

Motor Current (TEST INST.) Motor Voltage (TEST INST.) Recirc Flow (TEST INST.)

$\frac{\frac{2.6}{483} \text { Amps }}{\frac{18}{\sqrt{4}} \mathrm{pm}}$

Separator Inlet Pressure (LOCAL) 25 psi

Separator Outlet Pressure (LOCAL)

Test Engineer:

$\frac{10}{13 G K}$ psi

3.49 ALLOW Closed Loop Filtration Recirc Pump AZ101-W-P-1 to operate for one hour for collection of vibration data and bearing data. 
PREOPER .. IONAL TESTING, RECIRCULATION CO $\ldots$.NSER COOLING WHC. SD - W03O-POTP. OC

Revision No. 0

ATTACHMENT C

HNF-SD-W030-TD-003,

REV. O, PAGE 79

TANK AZ101 RECIRC CONDENSER

3.50 RECORD vibration data and bearing temperatures on Appendix D-5.

Test Engineer:

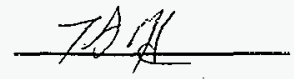

$3.51^{\circ}$ OPEN Bag Filter AZ101-W-F-2 inlet isolation valve HV-AZ101W-4.

3.52 VENT all air from the filter housing using the pressure/vacuum relief valve on top of the filter.

3.53 OPEN Bag Filter AZ101-W-F-2 outlet isolation valve HV-AZ101W-5.

3.54 VERIFY bag filter differential pressure indicates in the green range on PDIS-AZ101WF-2.

Test Engineer:

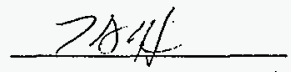

3.55 LOOSEN fitting on Bag Filter POIS-AZ101WF-2 low side instrument tubing.

3.56 VERIFY Bag Filter high differential pressure alarm PDAH-AZ101WF-2 initiates at 15psid on graphics screen 06EvpAY1.v.

Test Engineer:

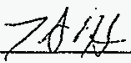

3.57. TIGHTEN fitting Bag Filter POIS-AZ101WF-2 low side instrument line.

3.58 VERIFY Bag Filter high differential pressure alarm PDAH-AZ101WF-2 clears on graphics screen 06EvpaY1.v.

Test Engineer:

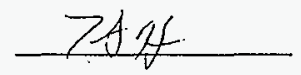

3.59 RECORD the conductivity reading indicated on the Clearview controller.

3.59.1 Tower fluid conductivity $\frac{60}{60}$ Micromhos

Test Engineer:

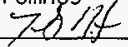

3.60 PLACE the Test Switch on the Clearview Controller in the TEST position.

3.61 VERIFY Evaporator Tower Bleed Valve EV-AZ101EWT-1A2 OPENS.

Test Engineer:

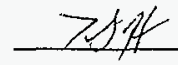


PREOPER .ONAL TESTING, RECIRCULATION CON _NSER COOLING WHC-SD-W030-POTP-C

Revision No.

ATTACHMENT $C$

HNF-SD-W030-TD-003,

REV. O, PAGE 80

TANK AZ101 RECIRC CONDENSEF

3.62 PLACE the Test Switch on the Clearview Controller in the OPER position.

3.63 VERIFY Evaporator Tower Bleed Valve EV-AZ101EWT-1A2 CLOSES.

Test Engineer:

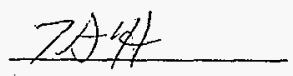

3.64 PLACE the Closed Loop Filtration System Recirc Pump AZ101-W-P-1 local control switch AZ101WP-1 in OFF.

3.65 CLOSE the Closed Loop Filtration System inlet and outlet block valves HV-AZ101W-1 and HV-AZ101W-3.

3.66 IF desired. THEN DRAIN the Closed Loop Filtration System as follows, OTHERWISE continue at step 3.67:

3.66.1 REMOVE drain plug from Closed Loop Filtration System Recirc Pump casing.

3.66.2 DISCONNECT hoses on either side of bag filter.

3.66.3 REMOVE vent plug from top of vent filter.

3.66.4 WHEN system completes draining. THEN reinstall all plugs and connect hoses to bag filter.

3.67 PLACE Evaporator Tower AZ101-EW-T-1 Spray Pump AZ101-EW-SP-1 remote control switch HS-AZ101-EWSP-1 in STOP.

3.68 VERIFY Evaporator Tower AZ101-EW-T-1 Spray Pump AZ101-EW-SP-1 STOPS.

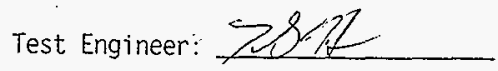

3.69 PLACE Evaporator Tower AZ101-EW-T-1 remote control switch HSAZ10I-EWT-1A1 in OFF.

3.70 VERIFY the following events occur:

3.70.1 AZ101 Evaporator Tower Cooling Fan STOPS.

3.70.2 AZ101 Evaporator Tower AZ101-EW-T-1 Temperature Control Damper OPENS.

Test Engineer:

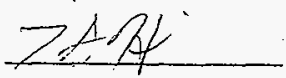

3.71 CLOSE Raw water supply to the Evaporator Tower HV-AZ101RW-1. 


\section{PREOPER$_{4}$ IONAL TESTING, RECIRCULATION CONL WHC.SD-W030-POTP-003 \\ Revision No. 0 \\ TANK AZ101 RECIRC CONDENSER CI \\ REV. 0, PAGE 81}

3.72 IF it is desired to drain the Evaporator Tower. THEN PERFORM the following steps, OTHERWISE continue at step 3.75 .

3.73 PLACE the Test Switch on the Clearview Controller in the TEST position to allow the Evaporator Tower to drain.

3.74 PLACE the Test Switch on the Clearview Controller in the OPER position.

3.75 VERIFY a1] test equipment installed to support this test has been removed.
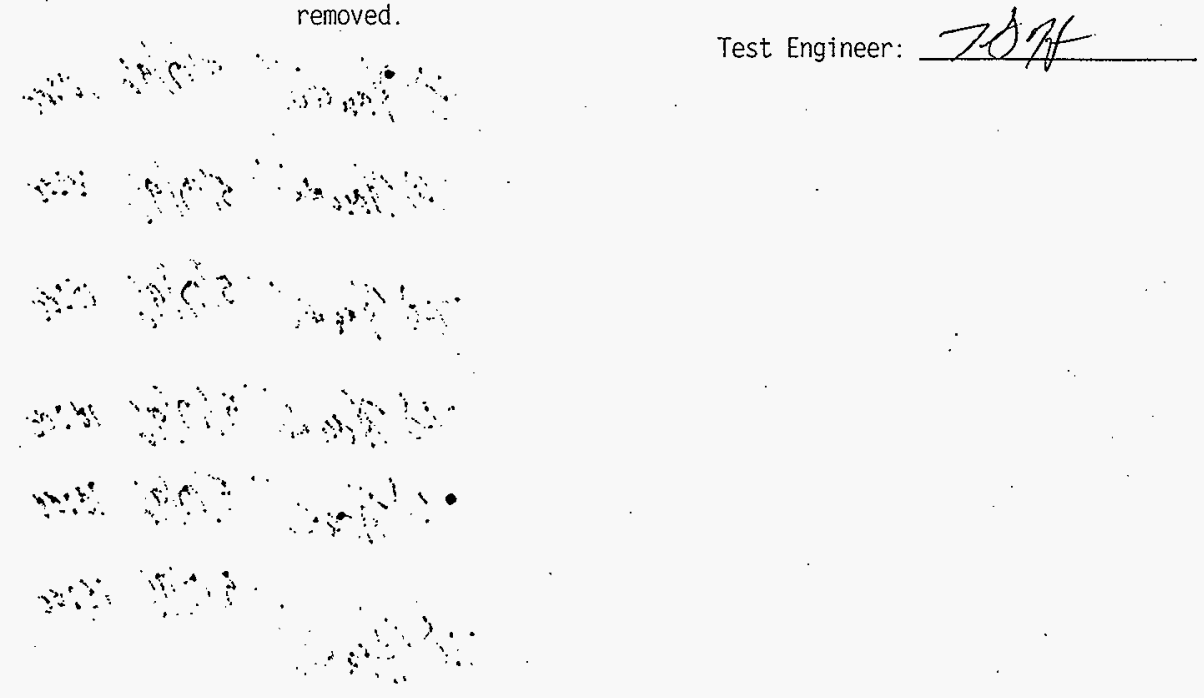


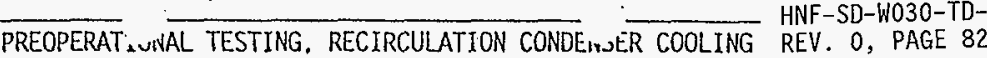
WHC.SD-W030-POTP-003

Revision No. 2

ATTACHMENT C

PA

Effective Date

TANK AZ101 RECIRC CONDENSER COOLING SYSTEM

\begin{tabular}{|c|c|c|c|}
\hline $\begin{array}{l}\text { Equipment } \\
\text { Number }\end{array}$ & Functional Description & Signature & Date/Time \\
\hline LSL-AZ101EWT-1 & $\begin{array}{l}\text { Az101 Evaporator Tower Pan Low } \\
\text { Level Switch on Evaporator Tower }\end{array}$ & $\pi=-1030-006$ & \\
\hline CD-AZ101EWT-I & $\begin{array}{l}\text { AZ101 Evaporator Tower Pan Fluid } \\
\text { Conductivity Element on } \\
\text { Evaporator Tower }\end{array}$ & Latell & $5 / 10 / 96 \cdot 10: 5$ \\
\hline PI -AZ101WS-1 & $\begin{array}{l}\text { Evaporator Tower Filtration Loop } \\
\text { Separator Inlet Pressure } \\
\text { Indicator }\end{array}$ & i: ots & $5 / 10 / 16 \cdot 10.0$ \\
\hline PI-AZ101WS-2 & $\begin{array}{l}\text { Evaporator Tower Filtration Loop } \\
\text { Separator Outlet Pressure } \\
\text { Indicator }\end{array}$ & hurell & $5 / 10 / 96$ w:on \\
\hline PDIS-AZ101WF-2 & $\begin{array}{l}\text { Evaporator Tower Filtration Loop } \\
\text { Bag Filter Differential Pressure } \\
\text { Indicating Switch }\end{array}$ & & $5 / 17 / 96 \quad 10: 80$ \\
\hline PI-AZ101EWP-IA1 & $\begin{array}{l}\text { Recirc Pump AZ101-EW-P-1A } \\
\text { Suction Pressure Indicator }\end{array}$ & & $5 / 17 / 9610.08$ \\
\hline PI-AZ101EWP-1A2 & $\begin{array}{l}\text { Recirc Pump AZ101-EW-P-1A } \\
\text { Discharge Pressure Indicator }\end{array}$ & Tofyouelf & $5 / 10 / 96 \quad 10: 08$ \\
\hline FT-AZ101EWR-1 & $\begin{array}{l}\text { A2101 Recirc Condenser Cooling } \\
\text { System Return Header Flow } \\
\text { Transmitter in return line at } \\
\text { Evaporator Tower. }\end{array}$ & treell & $5 / 13 / 9610: 00$ \\
\hline LSL-AZ101EWTK-1. & $\begin{array}{l}\text { Expansion Tank AZ101-EW-TK-1 Low } \\
\text { Level Switch }\end{array}$ & $\pi \cdot 1 / 030-006$ & \\
\hline LI-AZ101EWTK-1 & $\begin{array}{l}\text { Expansion Tank AZ101-EW-TK-I } \\
\text { Level Indicator }\end{array}$ & $T E-w 030-006$ & \\
\hline & & & \\
\hline
\end{tabular}


PREOPERATIONAL TESTING, RECIRCULATION IMMNENCED RMNI TNI ........ WHC - SD - WO3O-POTP-003

ATTACHMENT C

HNF-SD-W030-TD-003,

Revision No. 0

TANK AZ101 RECIRC CONDENSER COOL

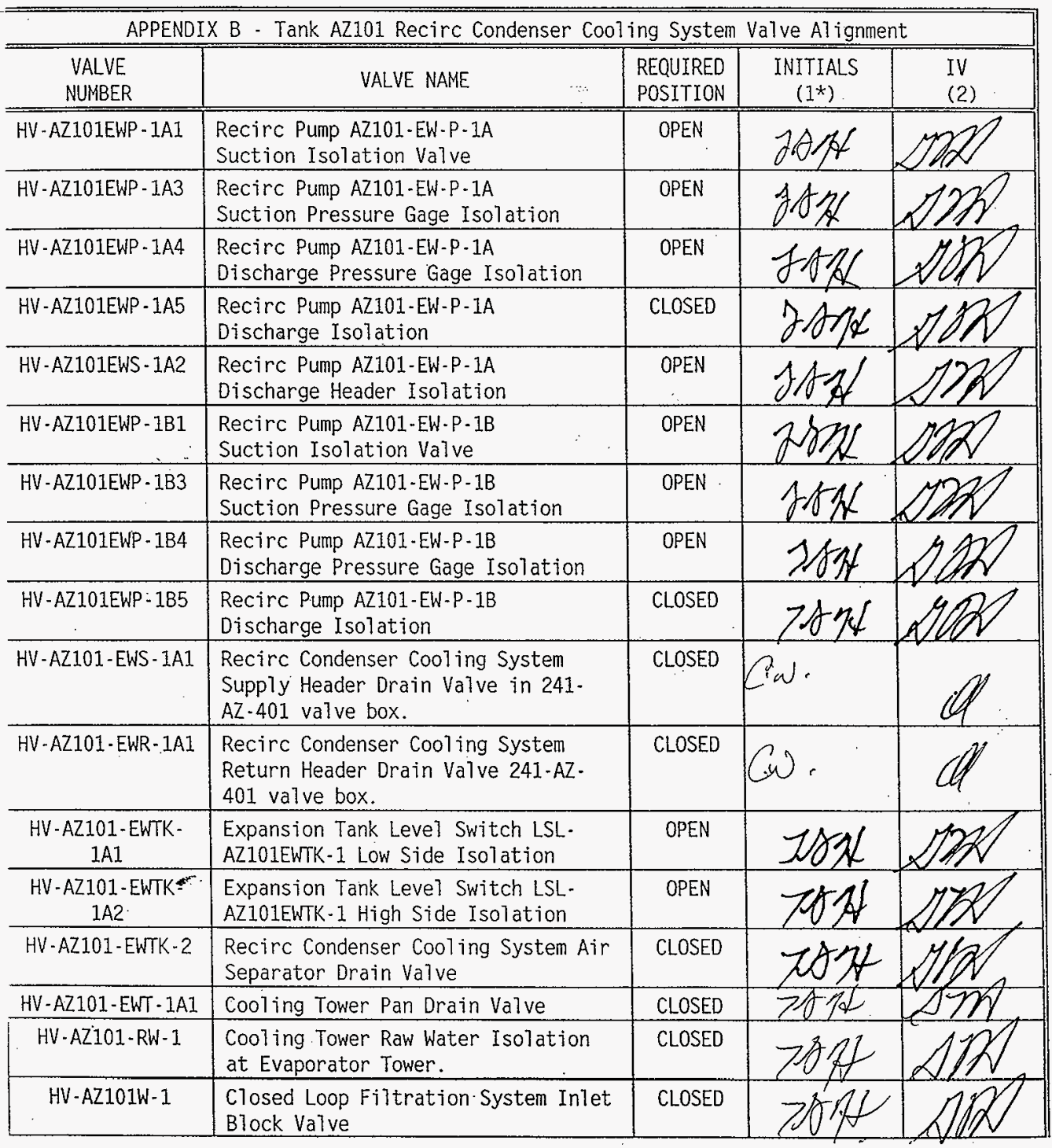


PREOPER IONAL TESTING, RECIRCULATION CO, .NSER COOLING WHC - SD - W030 - POTP - 003

Revision No. 0

ATTACHMENT C

TANK AZ101 RECIRC CONDENSER C

HNF-SD-W030-TD-003,

REV. O, PAGE 84

\begin{tabular}{|c|c|c|c|c|}
\hline \multicolumn{5}{|c|}{ APPENDIX B - Tank AZ101 Recirc Condenser Cooling System Valve Alignment } \\
\hline $\begin{array}{l}\text { VALVE } \\
\text { NUMBER } \\
\end{array}$ & VALVE NAME & $\begin{array}{l}\text { REQUIRED } \\
\text { POSITION } \\
\end{array}$ & $\begin{array}{c}\text { INITIALS } \\
\left(1^{*}\right) \\
\end{array}$ & $\begin{array}{l}\text { IV } \\
(2) \\
\end{array}$ \\
\hline HV-AZ101EWP-1A1 & $\begin{array}{l}\text { Recirc Pump AZ101-EW-P-1A } \\
\text { Suction Isolation Valve }\end{array}$ & OPEN & & r. 11 \\
\hline$H V \cdot A Z 101 E W P-1 A 3$ & $\begin{array}{l}\text { Recirc Pump AZ101-EW.P-1A } \\
\text { Suction Pressure Gage. Isolation }\end{array}$ & OPEN & & $97 x$ \\
\hline HV-AZ101EWP-1A4 & $\begin{array}{l}\text { Recirc Pump AZ101-EW-P-1A } \\
\text { Discharge Pressure Gage Isolation }\end{array}$ & OPEN & & \\
\hline HV-AZ101EWP-1A5 & $\begin{array}{l}\text { Recirc Pump AZ101-EW-P-IA } \\
\text { Discharge Isolation }\end{array}$ & CLOSED & & \\
\hline HV - AZ101EWS- $1 A 2$ & $\begin{array}{l}\text { Recirc Pump AZ101-EW-P-1A } \\
\text { Discharge Header Isolation }\end{array}$ & OPEN & & \\
\hline$H V \cdot A Z 101 E W P \cdot 1 B 1$ & $\begin{array}{l}\text { Recirc Pump AZ101-EW-P-1B } \\
\text { Suction Isolation Valve } \\
\end{array}$ & OPEN & & \\
\hline HV - AZ101EWP - .1B3 & $\begin{array}{l}\text { Recirc Pump AZ101-EW-P-1B } \\
\text { Suction Pressure Gage Isolation }\end{array}$ & OPEN & & \\
\hline HV-AZ101EWP-1B4 & $\begin{array}{l}\text { Recirc Pump AZ101-EW-P.1B } \\
\text { Discharge Pressure Gage Isolation }\end{array}$ & OPEN & & \\
\hline HV -AZ101EWP-1B5 & $\begin{array}{l}\text { Recirc Pump AZ101-EW-P-1B } \\
\text { Discharge Isolation }\end{array}$ & CLOSED & & \\
\hline$H V \cdot A Z 101-E W S-1 A 1$ & $\begin{array}{l}\text { Recirc Condenser Cooling System } \\
\text { Supply Header Drain Valve in } 241 \text { - } \\
\text { AY.401 valve box. }\end{array}$ & CLOSED & & \\
\hline$H V \cdot A Z 101 \cdot E W R \cdot 1 A 1$ & $\begin{array}{l}\text { Recirc Condenser Cooling System } \\
\text { Return Header Drain Valve 241-AY. } \\
401 \text { valve box. }\end{array}$ & CLOSED & & \\
\hline $\begin{array}{l}\text { HV-AZ101-EWTK- } \\
\text { IAI } \\
\end{array}$ & $\begin{array}{l}\text { Expansion Tank Level Switch LSL- } \\
\text { AZ101EWTK-1 Low Side Isolation } \\
\end{array}$ & OPEN & & \\
\hline $\begin{array}{c}\text { HV-AZ101-EWTK- } \\
1 A 2\end{array}$ & $\begin{array}{l}\text { Expansion Tank Level Switch LSL- } \\
\text { AZ101EWTK-1 High Side Isolation }\end{array}$ & OPEN & & \\
\hline HV - AZ101 - EWTK-2 & $\begin{array}{l}\text { Recirc Condenser Cooling System Air } \\
\text { Separator Drain Valve }\end{array}$ & CLOSED & & rytin \\
\hline HV - AZ101-EWT - 1A1 & Cooling Tower Pan Drain Valve & CLOSED & & (x) \\
\hline$H V-A Z 101-R W-1$ & $\begin{array}{l}\text { Cooling Tower Raw Water Isolation } \\
\text { at Evaporator Tower. }\end{array}$ & CLOSED & $28 \lambda$ & MIN \\
\hline$H V \cdot A Z 101 W \cdot I$ & $\begin{array}{l}\text { Closed Loop Filtration System Inlet } \\
\text { Block Valve. }\end{array}$ & CLOSED & $28 \&$ & HID \\
\hline
\end{tabular}


PREOPERATL .AL TESTING, RECIRCULATION CONDEI WHC-SD-WO30-POTP-00:

Revision No.

ATTACHMENT C

HNF-SD-W030-TD-003,

REV. O, PAGE 85

TANK AZ101 RECIRC CONDENSER C

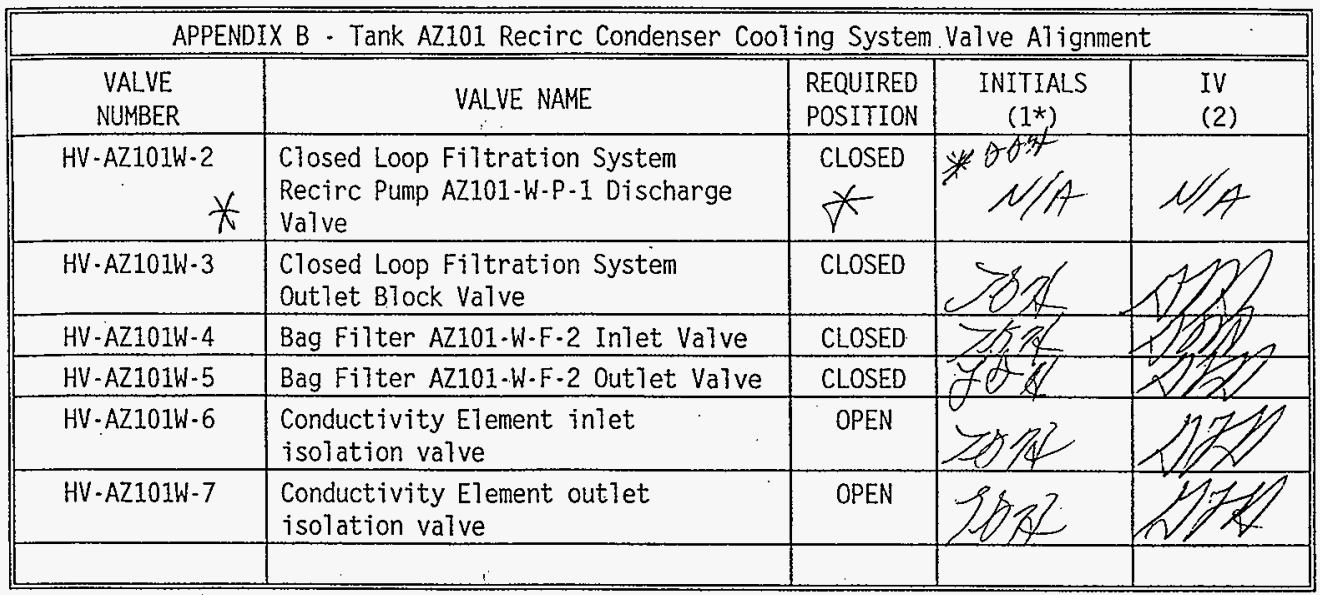

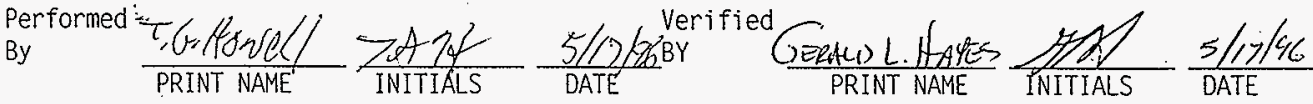

* nute addep to reprort.

VALVE HU-AZIOIW- 2 WAS

DELETED BY ECN-W-O30-215.

a.".

COPY ENCLSED IN ATT. 3.

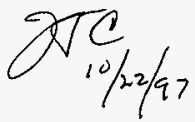


PREOPERn, .ONAL TESTING, RECIRCULATION CON ISER COOLING WHC-SD-W030-POTP-003

Revision No. 0 ATTACHMENT C TANK AZ101 RECIRC CONDENSER CC

HNF-SD-W030-TD-003, REY. 0, PAGE 86

\begin{tabular}{|c|c|c|c|c|}
\hline $\begin{array}{c}\text { BREAKER } \\
\text { NUMBER }\end{array}$ & BREAKER NAME AND LOCATION & $\begin{array}{l}\text { REQUIRED } \\
\text { POSITION }\end{array}$ & $\begin{array}{c}\text { INITIALS } \\
\left(1^{*}\right)\end{array}$ & $\begin{array}{l}\text { IV } \\
\text { (2) }\end{array}$ \\
\hline $1 \mathrm{~A}$ & $\begin{array}{l}\text { Evaporator Tower AZ101 Supply } \\
\text { Breaker in Distribution Panelboard } \\
\text { PP.2 Diesel Generator Building }\end{array}$ & ON & & \\
\hline 2 HP SEPARATOR & $\begin{array}{l}\text { Evaporator Tower AZ101 Separator } \\
\text { Recirc Pump Supply Breaker in Local } \\
\text { Panel AZ101-EVAP-TWR }\end{array}$ & OFF & & \\
\hline $\begin{array}{l}\text { FLUID COOLER } \\
\text { CONTROL PANEL } \\
\text { AZ101-EW-T-1 }\end{array}$ & $\begin{array}{l}\text { Evaporator Tower AZ101 Fan, Recirc } \\
\text { Pump, Pan Heater Supply Breaker in } \\
\text { Local Pane] AZ101-EVAP-TWR }\end{array}$ & OFF & & \\
\hline $\begin{array}{l}\text { RECIRC PUMP } \\
\text { AZ101-EW-P-IA }\end{array}$ & $\begin{array}{l}\text { AZ101 Recirc Condenser Cooling } \\
\text { System Recirc Pump AZ101-EW-P-1A in } \\
\text { Local Panel AZ101-EVAP-TWR }\end{array}$ & OFF & & \\
\hline $\begin{array}{l}\text { RECIRC PUMP } \\
\text { AZ101-EW-P-1B }\end{array}$ & $\begin{array}{l}\text { AZ101 Recirc Condenser Cooling } \\
\text { System Recirc Pump AZ101-EW-P-1B in } \\
\text { Local Panel AZ101-EVAP-TWR }\end{array}$ & OFF & & \\
\hline $\begin{array}{l}\text { PANEL PP.X8 } \\
7894 \\
51016\end{array}$ & $\begin{array}{l}\text { Evaporator Tower Panelboard PP.7 } 8 \\
\text { Supply Breaker in Local Panel } \\
\text { AZ102-EVAP:TWR } \\
\end{array}$ & ON & & \\
\hline $\begin{array}{l}\text { Disconnect } \\
\text { switch }\end{array}$ & $\begin{array}{l}\text { Evaporator Tower AZ101 Separator } \\
\text { Recirc Pump local disconnect switch } \\
\text { at pump. }\end{array}$ & OFF & & \\
\hline & & & & \\
\hline & & & & \\
\hline & & & & \\
\hline & & & & \\
\hline ar & & & & \\
\hline & & & & \\
\hline
\end{tabular}

Performed By

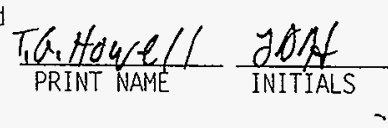

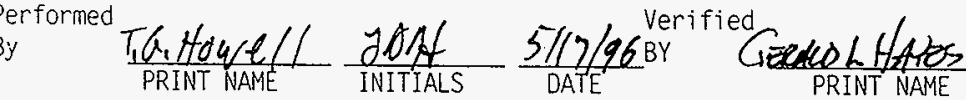

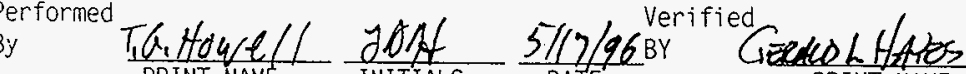
PRINT NAME

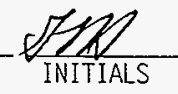
$\frac{5 / m / 96}{\text { DATE }}$ 
PREOPERAT AL TESTING. RECIRCULATION CONDI :R COOLING HNF-SD-W030-TD-003 WHC-SD-W030-POTP-003

Revision No. 0

ATTACHMENT C Effective Di

PAI REV. 0, PAGE 87

Effecti

TANK AZ101 RECIRC CONDENSER COOLING SYSTEM

APPENDIX D-1

OPERATION DATA SHEET

Equipment Name: TANK AZ101 RECIRC CONDENSER COOLING SYSTEM RECIRC PUMP

EQUIPMENT I.D. NO: : AZ101-EW-P-IA

NAMEPLATE DATA:

See Appendix F. $\overline{7}$

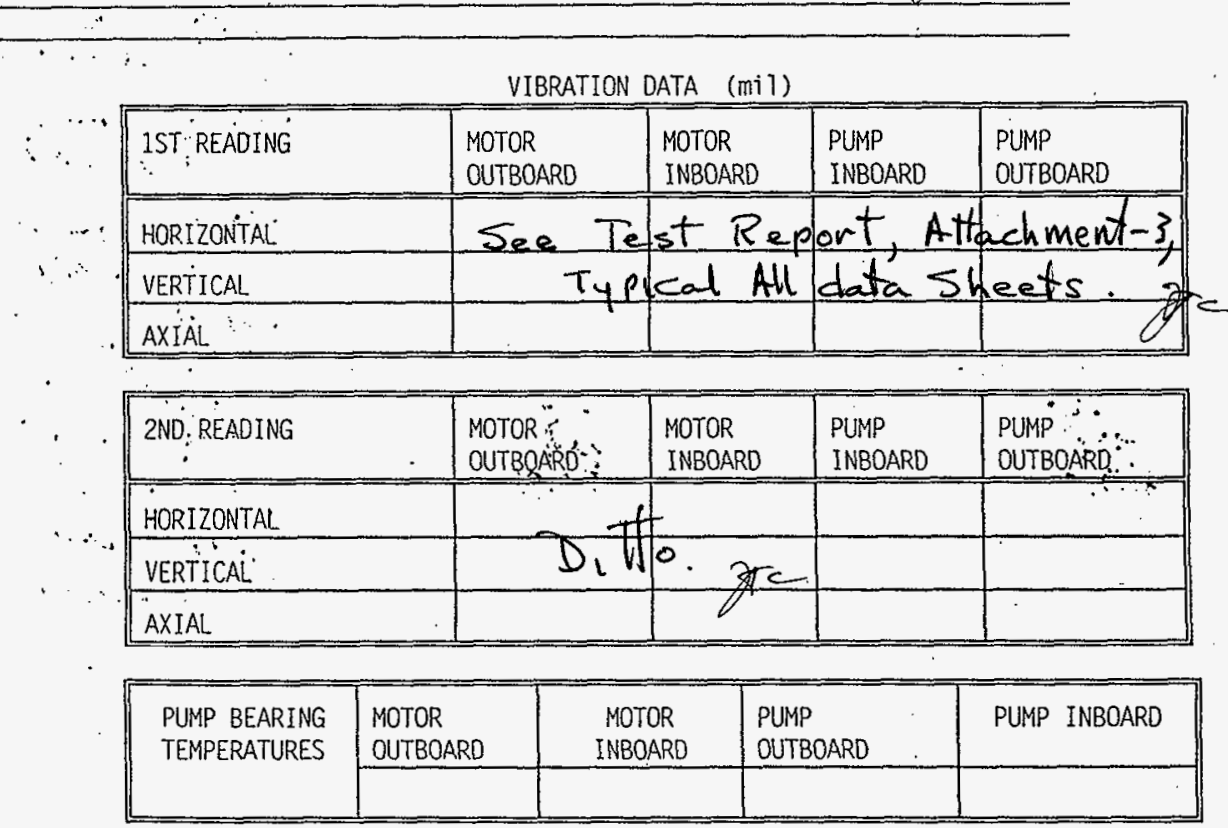

REMARKS:

Performed by :

Date

$\therefore$ a verifiedtio:

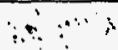

$\therefore$ 
PREOPERAT _. WHC.SD-W030-POTP.003

Revision No. 0 ATTACHMENT C

HNF-SD-W030-TD-003,

TANK AZ101 RECIRC CONDENSER COC

REV. 0, PAGE 88

\section{APPENDIX $F$}

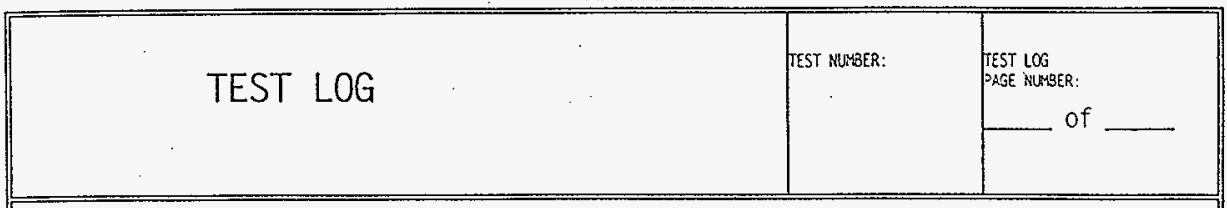

TEST TITLE:

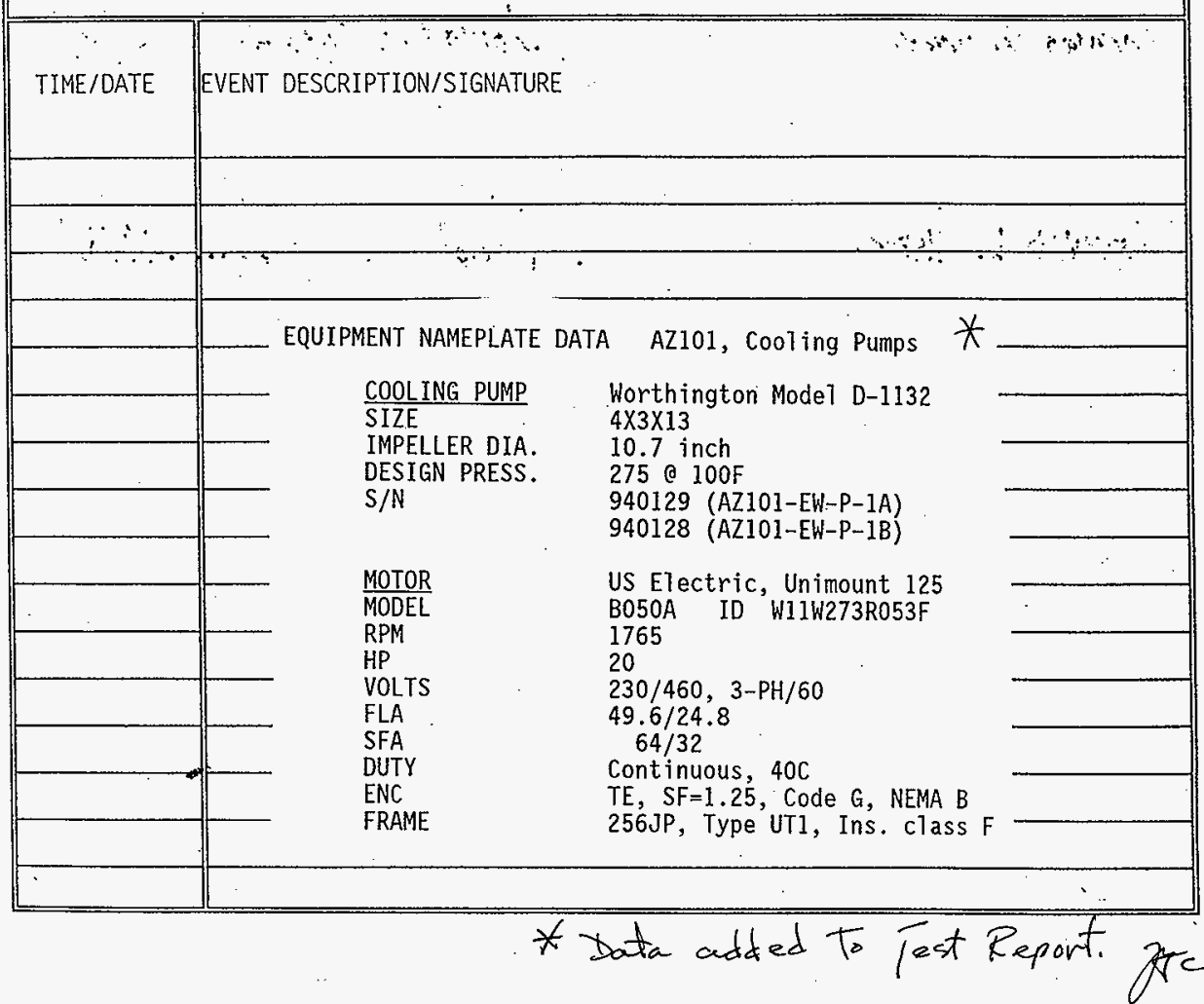


PREOPER .ONAL TESTING. RECIRCULATION CON. NSER COOLING WHC-SD-W030-POTP-003

Revision No. @ 0

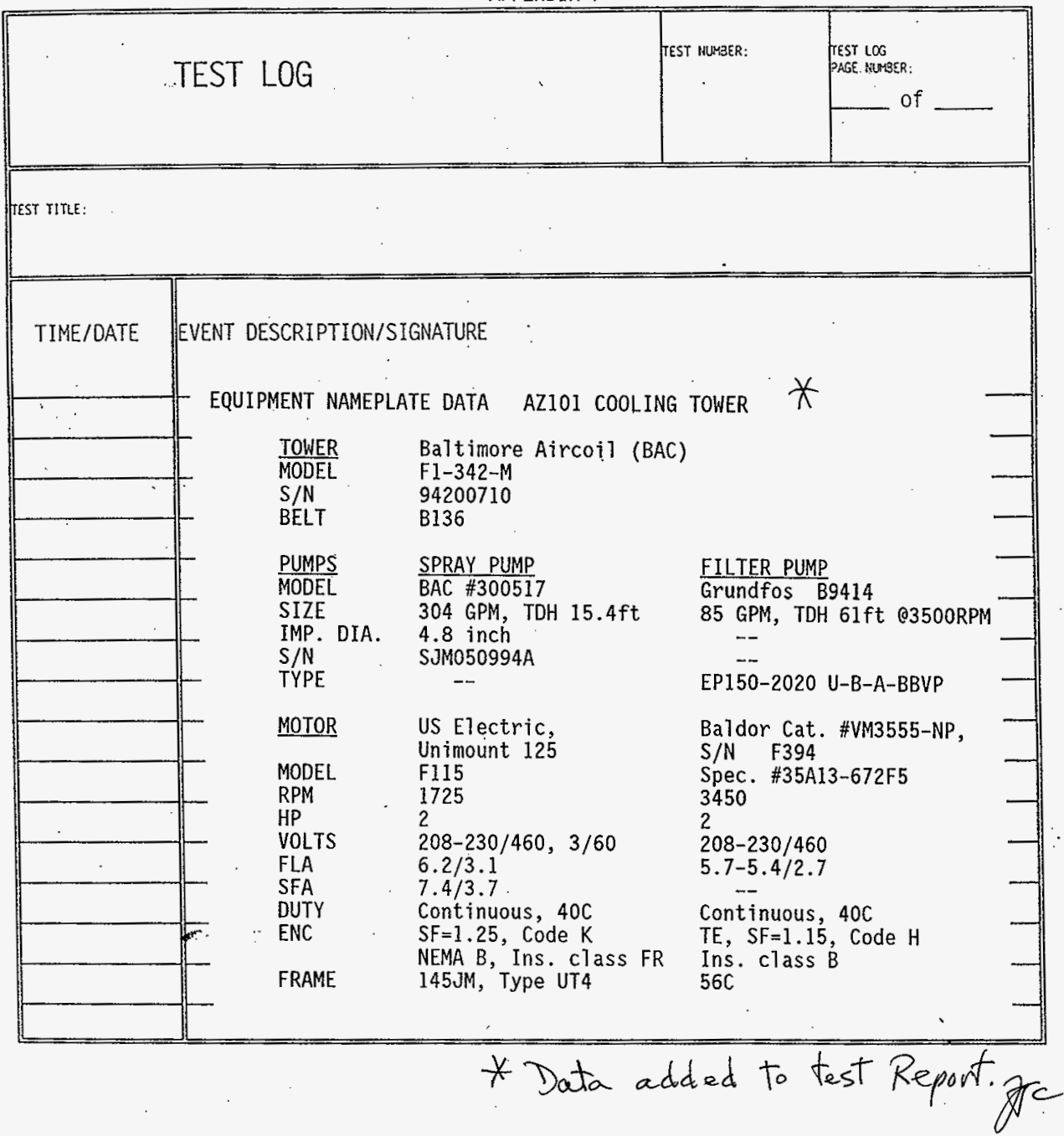


PREOPEK SONAL TESTING, RECIRCULATION COI. NSER COOL WHC-SD-W030-POTP- 003

Revision No. 0

ATTACHMENT C Effecti:.....

HNF-SD-W030-TD-003,

REV. 0, PAGE 90

\section{APPENDIX G}

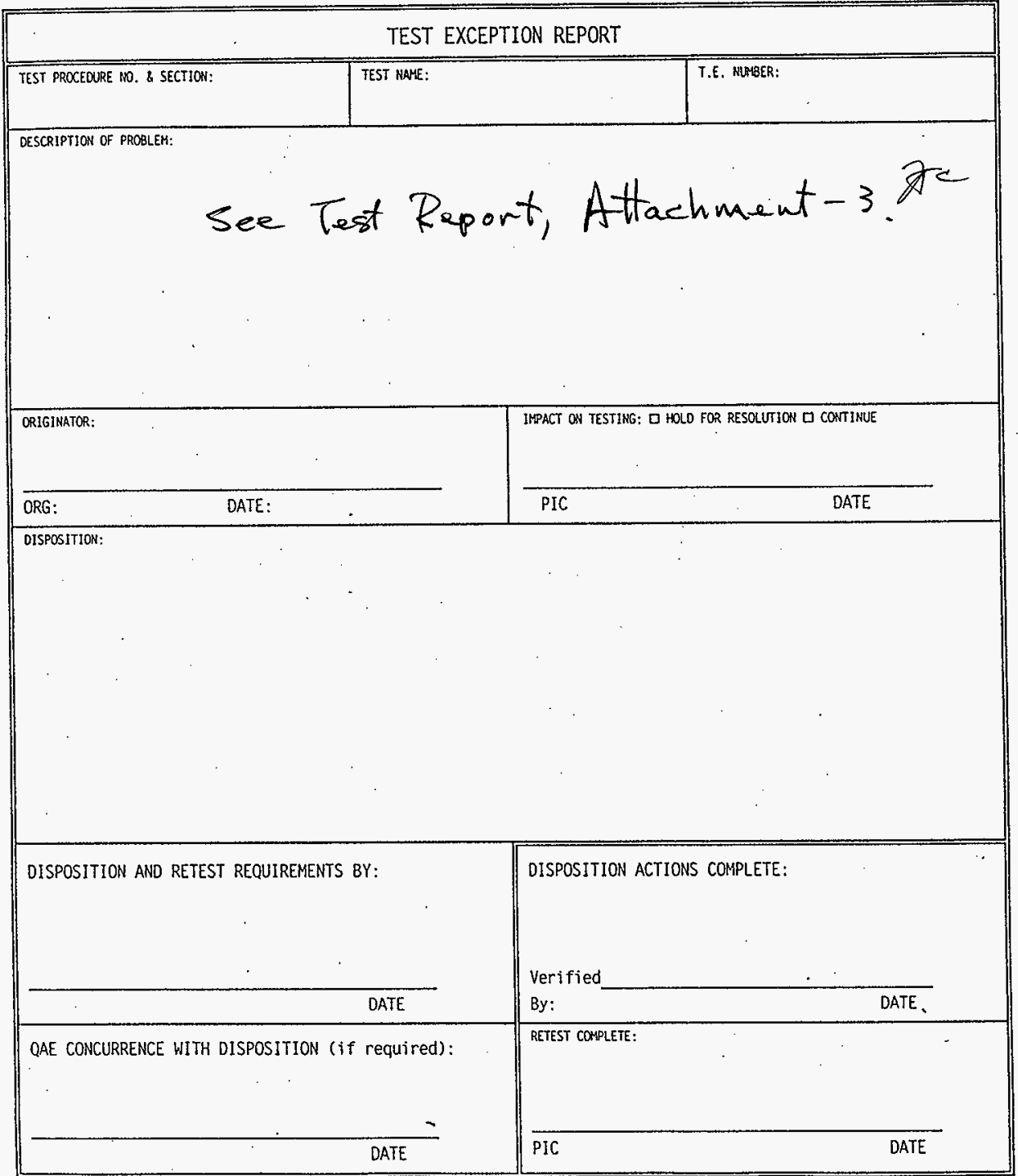




\subsection{INITIAL CONDITIONS}

1.1 VERIFY all system instrumentation in Appendix $A$ is calibrated and has a current calibration tag affixed to each instrument and that all loop calibrations are complete.

Test Engineer:

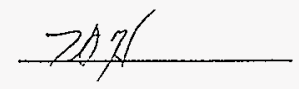

1.2 VERIFY the system is aligned for preoperational testing in accordance with Appendix B.

Test Engineer:

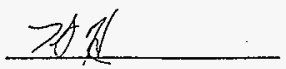

1.3 VERIFY system electrical circuit breakers are aligned in accordance with Appendix C.

Test Engineèr:

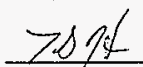

1.4 VERIFY expansion tank level is at midlevel as indicated on LIAZ102EWTK-1.

Test Engineer:

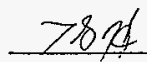

1.5 All personnel who will be involved with this procedure have provided the required signature verification information in Appendix $\dot{E}$.

Test Engineer:

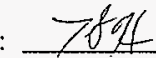

1.6 The test engineer has verified, by review of the $\operatorname{tag} \log$ and a walkdown of the systems being tested, that all components within and including the test boundary have been "blue" tagged.

Test Engineer:

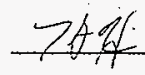

\subsection{Tank AZ102 Glycol Recirc System Testing}

2.1 VERIFY Recirc Pump AZ102-EW-P-1A local control switch HS-AZ102EWPAAl in the STOP position.

Test Engineer:

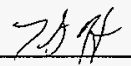

2.2 CLOSE local breaker for Recirc Pump AZ102-EW-P-1A. 
PREOPERATI AL TESTING, RECIRCULATION CONDE, 2 COOLING WHC-SD-W030-POTP-00:

Revision No. $\underline{0}$

ATTACHMENT D

HNF-SD-W030-TD-003, REV. O, PAGE 92

TANK AZ102 RECIRC CONDENSER (

2.3 RECORD the following data for Recirc Pump AZ102-EW-P-1A:

Expansion Tank Level (Local)

Suction Pressure(Local)

8 psi

Run Time (graphics screen 06EvpAY1.v) _L, 0 hrs

Test Engineer:

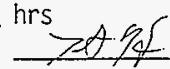

2.4 ATTEMPT to start Recirc Pump AZ102-EW-P-1A from MCS graphics screen 06EvpAYl.v.

2.5 VERIFY Recirc Pump AZ102-E்-P-1A does not start.

Test Engineer:

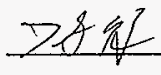

2.6 BUMP Recirc Pump AZ102-EW-P-1A by placing control switch HSAZI02EWP-1Ai in START and then back to STOP.

2.7 VERIFY rotation of the Recirc Pump AZ102-EW-P-1A is in the direction indicated by the arrow on the pump.

Test Engineer:

2.8 PLACE Recirc Pump AZ102-EW-P-1A local control switch HS-AZ102EWPIAl in the START position.

2.9 VERIFY Recirc Pump AZ102-EW-P-1A starts.

Test Engineer:

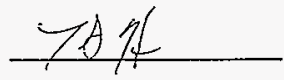

2.10 RECORD the following data for Recirc Pump AZ102-EW-P-IA:

2.10.1 Motor Current (graphics screen 06EvpAY1.v) 7.3 Amps

2.10.2 Motor voltage (TEST INST.) 485 volts

2.10.3 Suction Pressure (LOCAL) $\frac{8}{86}$ psi

2.10.4 Discharge Pressure (LOCAL)

Test Engineer:

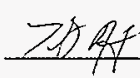

2.11 SLOWLY OPEN Recirc pump AZ102-EW-P-1A discharge vaive HV-AZ102EWP$1 A 5$ and establish a system flowrate of $2192 \mathrm{gpm}$ as indicated on FI-AZ102EWR-1 on graphics screen 06EvpAY1.V. 
PREOPEK, IONAL TESTING, RECIRCULATION CONL. NSER COOLING WHC-SD-W030-POTP-00:

Revision No. 0 ATTACHMENT D

HNF-SD-W030-TD-003,

TANK AZ102 RECIRC CONDENSER I

REV. O, PAGE 93

2.12 VERIFY low flow alarm FAL-AZ102WR-1 clears graphics screen 06EvPAY1.V.

Test Engineer: $\quad$ ZAP

2.13 RECORD the following data for Recirc Pump AZ102-EW-P-1A:

2.13.1 Motor Current (graphics screen 06EvpAY1.v) 9.5

2.13.2 Motor voltage (TEST INST.) 483 volts

2.13.3 Suction Pressure (LOCAL) 5 psi

2.13.4 Discharge Pressure (LOCAL)

2.13.5 System Flow (graphics screen 06EvpAY1.v) /92 gpm

2.13.6. Expansion Tank Level (Local) $1 / 2$

Test Engineer:

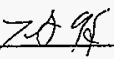

2.14 THROTTLE CLOSED valve HV-AZ102EWP-1A2 unti1 system flow starts to decrease as indicated on FI-AZ102EWR-1 graphics screen 06EvPAY1.V.

2.15 FULLY OPEN Recirc Pump AZ102-EW-P-1A discharge valve HV-AZ102EWP$1 \mathrm{~A} 5$.

2.16 ADJUST system flow to z192 gpm using HV-AZ102EWP-1A2

2.17 VERIFY Recirc Pump AZ102-EW-P-1B local control switch HS-AZ102EWP181 in the STOP position.

- Test Engineer:

2.18 CLOSE - local breaker for Recirc Pump AZ102-EW-P-1B.

2.19 RECORD the following data for Recirc Pump AZ102-EW-P-1B:

Suction Pressure(Local) $\quad 6$ psi

Run Time (graphics screen 06EvpAY1.v) $O$ hrs.

Test Engineer:

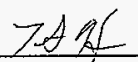




\section{PREOPERAT_ UIVAL TESTING, RECIRCULATION INNAF WHC-SD-W030-POTP-00:}

Revision No. 0 $\therefore$ R rnOI TNR

HNF-SD-W030-TD-003, REV. O, PAGE 94 TANK AZ102 RECIRC CONDENSER $i$

2.22 ATTEMPT to start Recirc Pump AZ102-EW-P-1B from MCS graphics screen 06EvpAY1.v.

2.23 VERIFY: Recirc Pump AZ102-EW-P-1B does not start.

Test Engineer:

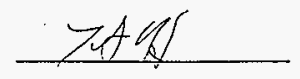

2.24 PLACE Recirc Pump AZ102-EW-P-1B local control switch HS-AZ102EWP$1 B 1$ in the START position.

2.25 VERIFY Recirc Pump AZ102-EW-P-1B starts. Test Engineer:

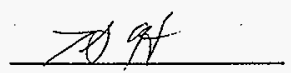

2.26 RECORD the following data for Recirc Pump AZ102-EW-P-1B:

2.26.1 Motor Current (graphics screen 06EvpAY1.v) $\geqslant .3$

2.26.2 Motor voltage (TEST INST.) 483 Volts

2.26.3 Suction Pressure (LOCAL) $\frac{6}{6}$ psi

2.26.4 Discharge Pressure (LOCAL) 《\# psi

Test Engineer:

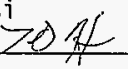

2.27 SLOWLY OPEN Recirc pump AZ102-EW-P-1B discharge vaTve HV-AZ102EWP1 B5.

2.28 ATTEMPT to stop Recirc Pump AZ102-EW-P-1A from MCS graphics screen 06EvPAY1.v.

2.29 VERIFY that Recirc Pump AZ102-EW-P-1A does not STOP.

Test Engineer:

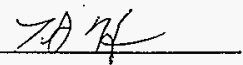

2.30 PLACE RecirC Pump AZ102-EW-P-1A local control switch HS-AZ102EWPIAl in the STOP pOsition.

2.31 VERIFY that Recirc Pump AZ102-EW-P-1A STOPS.

Test Engineer:

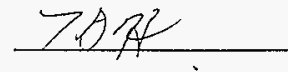

2.32 ADJUST system flow, if required, to 2192 gpm using HV-AZ102EWP$1 \mathrm{~A} 2$. 
PREOPER . IONAL TESTING, RECIRCULAT!

WHC.SD-W030-POTP-0O

Revision No. 0

ATTACHMENT D

HNF-SD-W030-TD-003,

REV. 0, PAGE 95

TANK AZ102 RECIRC CONDENSER .

2.33 RECORD the following data for Recirc Pump AZ102-EW-P-1B:

2.33.1 Motor Current (graphics screen 06EvpAY1.v) 9.5 Amps

2.33 .2

2.33 .3

2.33 .4

Motor Voltage (TEST INST.) 483 Volts

2.33 .5

2.33 .6

Suction Pressure (LOCAL)

Discharge Pressure (LOCAL)

System Flow (graphics screen 06EvpAYl.v) $201 \mathrm{gpm}$

Expansion Tank Level (Loca1)

Test Engineer:

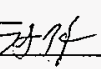

2.34 THROTHLE HV-AZ102EWP-1A2 to decrease flow to $<170 \mathrm{gpm}$.

2.35 VERIFY system low flow alarm. FAL-AZ102EWR-1 annunciates on graphics screen 06EvpAY1:v.

Test Engineer:

2.36 THROTTLE HV-AZ102EWP-IA2 to increase flow to $\geq 192 \mathrm{gpm}$.

2.37 VERIFY system low flow alarm. FAL-AZ102EWR-1 clears on graphics screen 06EvpAYl.v.

Test Engineer:

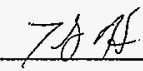

2.38 PLACE Recirc Pump AZ102-EW-P-1A local control switch HS-AZ102EWP$1 \mathrm{Al}$ in the REMOTE position.

2.39 START Recirc Pump AZ102-EW-P-1A from graphics screen 06EvpAY1.V

2.40 VERIFY Recirc Pump AZ102-EW-P-1A starts.

Test Engineer:

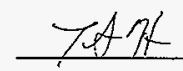

2.41 PLACE Recirc Pump AZ102-EW-P-1B local control switch HS-AZ102EWP$1 B 1$ in the STOP position.

2.42 VERIFY Recirc Pump AZ102-EW-P-1B stops.

Test Engineer:

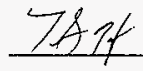

2.43 PLACE Recirc Pump AZ102-EW-P-1B local control switch HS-AZ102EWP$1 \mathrm{B1}$ in the REMOTE position.

2.44 PLACE Recirc Pump AZ102-EW-P-1B in Standby on graphics screen 06EvPAY1.V. 
PREOPERAT „AL TESTING, RECIRCULATION CONNF WHC-SD-W030-POTP-003

Revision No. 0
ATTACHMENT D

TANK AZ102 RECIRC CONDENSER CC
R rnNI TNR

HNF-SD-W030-TD-003, REV. O, PAGE 96

2.45 OPEN local breaker switch for Recirc Pump AZ102-EW-P-1A.

2.46 VERIFY Recirc Pump AZ102-EW-P-1B auto starts. Test Engineer:

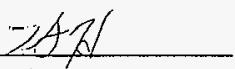

2.47 PLACE Recirc Pump AZ102-EW-P-1A remote control switch HS-AZ102EWP$1 A$ in STOP position on graphics screen 06EvpAY1.v.

2.48 CLOSE local breaker for Recirc Pump AZ102-EW-P-1A.

2.49 PLACE Recirc Pump AZ102-EW-P-1A in the Standby on graphics screen 06EvpAY1.V.

2.50 OPEN 1ocal breaker for Recirc Pump AZ102-EW-P-1B.

2.51. VERIFY Recirc Pump AZ102-EW-P-1A auto starts. Test Engineer:

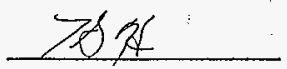

-2.52 PLACE Recirc Pump AZ102-EW-P-1B remote control switch HS-AZ102EWP18 in STOP position on graphics screen 06EvpAYl.v.

2.53 CLOSE local breaker for Recirc Pump AZ102-EW-P-1B.

2.54 ALLOW Recirc Pump AZ102-EW-P-1A to operate for one hour for collection of vibration data and bearing data.

2.55 RECORD vibration data and bearing temperatures on Appendix $0-1$.

Test Engineer:

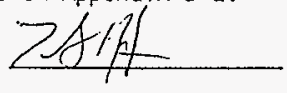

2.56 START Recirc Pump AZ102-EW-P-1B remotely from LCU4-17.

2.57 STOP Recirc Pump AZ102-EW-P-1A remotely from LCU4-16.

2.58 ALLOW Recirc Pump AZ102-EW-P-1B to operate for one hour for collection of vibration data and bearing data.

2.59 RECORD vibration data and bearing temperatures on Appendix D-2.

Test Engineer:

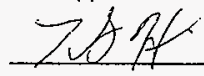




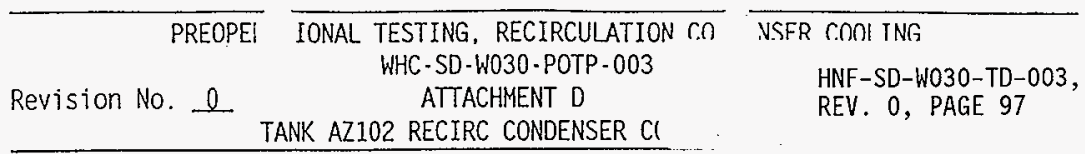

2.60 SLOWLY CLOSE Expansion Tank Leve1 Switch LSL-AZ102EWTK- 1 Low Side Isolation Valve HV-AZ102EWTK-IAl.

2.61. VERIFY Expansion Tank Low Level Alarm LAL-AZ102EWTK-1 annunciates on graphics screen 06EvpAY1.v.

Test Engineer:

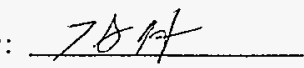

2.62 OPEN Expansion Tank Level Switch LSL-AZ102EWTK- 1 Low Side Isolation Valve HV-AZ102EWTK-1A1.

2.63 VERIFY Expansion Tank Low Level Alarm LAL-AZ102EWTK-1 clears on graphics screen 06EvpAY1.v.

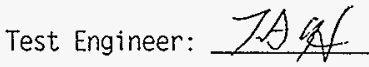

2.64 PLACE Low Flow Shutdown switches HS-AZ102EWP-1AB and HS-AZ102EWPIBB in the OVERRIDE position on graphics screen 06EvpAY1.V.

2.65 PLACE Recirc Pump AZ102-EW-P-1A in STANDBY on graphics screen 06EvpAYl.v.

2.66 THROTTLE HV-AZ102EWP-1A2 to decrease flow to <100 gpm.

2.67 VERIFY the Recirc Pump AZ102-EW-P-1B does not STOP on low flow.

Test Engineer:

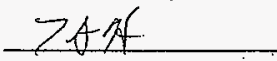

2.68 PLACE LOW Flow Shutdown Override switch HS-AZ102EWP-1BB in the NORMAL position from the LOI.

2.69 VERIFY the Recirc Pump AZ102-EW-P-1B STOPS on low flow.

$$
\text { Test Engineer: ZA }
$$

2.70 VERIFY RecirC PUmp AZ102-EW-P-1A starts.

Test Engineer:

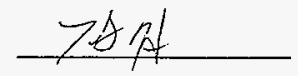

2.71 PLACE Low Flow Shutdown Override switch HS-AZ102EWP-1AB in the NORMAL position on graphics screen 06EvpAY1.v.

2.72 VERIFY the Recirc Pump AZ102-EW-P-1A STOPS on low flow.

Test Engineer:

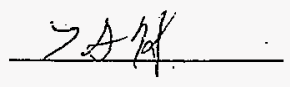




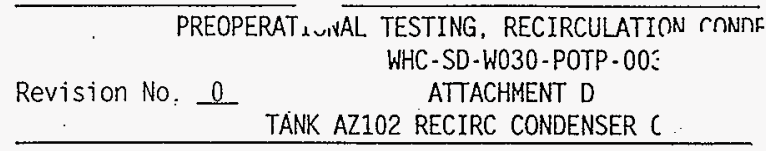

FR TONI TNG

HNF-SD-W030-TD-003, TANK AZ102 RECIRC CONDENSER C

REV. 0, PAGE 98

2.73. PLACE Low Flow Shutdown Override switch HS-AZ102EWP-1AB in the OVERRIDE position on graphics screen O6EvPAY1.V.

2.74 RESET Recirc Pump AZ102-EW-P-1A by placing remote control switch HS-AZ102EWP-1A in STOP position on graphics screen 06EvpAY1.V.

2.75 PLACE Recirc Pump AZ102-EW-P-1A remote control switch HS-AZ102EWPIA in START position on graphics screen 06EvPAY1.V.

2.76 VERIFY that Recirc Pump AZ102-EW-P-1A starts.

Test Engineer:

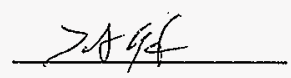

2.77 THROTTLE HV-AZ102EWP-1A2 to increase system flow to $2192 \mathrm{gpm}$.

2.78 PLACE LOW Flow Shutdown Override switch HS-AZ102EWP-1AB in the NORMAL position on graphics screen 06EvPAY1.v.

3.0 Tank AZ102 Evaporator Tower Testing

3.1 REMOVE manway covers on the cooling tower.

3.2 OPEN HV-AZ102-RW-I Cooling Tower Raw Water Isolation valve at Evaporator Tower.

3.3 VERIFY cooling tower fill via makeup valve EV-AZ102EWT-1A1.

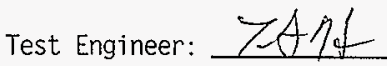

3.4 INSTALL manway covers when cooling tower fill is complete.

3.5 VERIFY the following local switch positions:

- Evaporator Tower AZ102-EW-T-1 Spray Pump AZ102-EW-SP-1 local control switch HS-AZ102-EWSP-1A in OFF.

- Evaporator Tower AZ102-EW-T-1 Fan local control switch HSAZ102EWT-1A1 in OFF.

- Evaporator Tower AZ102-EW-T-1 Pan Heater local control switch HS-AZ102EWT-1A 2in OFF.

Test Engineer:

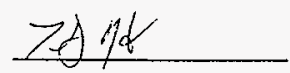

3.6 CLOSE the local supply breaker for the spray pump, cooling fan, and pan heater in the Evaporator Tower local distribution panel. 
3.7 REMOVE splash guard from top of Spray Pump AZ102-EW-SP-1 motor to enable viewing of the motor shaft.

3.8. BUMP Spray Pump AZ102-EW-SP-1 by placing control switch HS-AZ102EWSP-1A in START and then back to OFF.

3.9 VERIFY rotation of the Spray Pump AZ102-EW-SP-1 is in the direction indicated by the arrow on the pump.

Test Engineer:

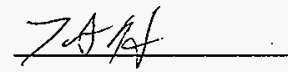

3.10. REINSTALL splash guard on Spray Pump AZ102-EW-SP-1 motor.

3.11 PLACE Evaporator Tower AZ102-EW-T-1 Spray Pump AZ102-EW-SP-1 local control switch HS-AZ102-EWSP-1A in START.

3.12 VERIFY Spray Pump AZ102-EW-SP-1 starts.

Test Engineer:

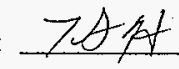

3.13 RECORD the following data for Spray Pump AZ102-EW-SP-1:

3.13.1 Motor Current (TEST INST.) $\quad 1.4$ Amps

3.13.2 Motor voltage (TEST INST.) $4 \overline{90}$ volts

3.13.3 Spray Flow (TEST INST.) 1/ff gpm

Test Engineer:

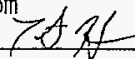

3.14 ALLOW Spray Pump AZ102-EW-SP-1 to operate for one hour for collection of vibration data and bearing data.

3.15 RECORD vibration data and bearing temperatures on Appendix D-3.

Test Engineer:

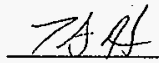

3.16 PLACE Evaporator Tower AZ102-EW-T-1 Spray Pump AZ102-EW-SP-1 remote control switch HS-AZ102-EWSP-1 in STOP on graphics screen 06EvpAY1.v.

3.17 VERIFY Spray Pump AZ102-EW-SP-1 does NOT STOP.

Test Engineer:

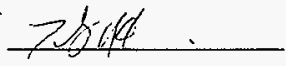

3.18 PLACE Evaporator Tower AZ102-EW-T-1 Temperature Control Damper local control Switch HS-AZ102-EWT-1A3 in OPEN. 
PREOPERAi IAL TESTING, RECIRCULATION CONDL ER COOLING WHC-SD-W030-POTP-003

Revision No. 0

TANK AZ102 RECIRC CONDENSER C

3.19 VERIFY Evaporator Tower AZ102-EW-T-1 Temperature Control Damper OPENS.

. Test Engineer: ZA D.L.

3.20 PLACE Evaporator Tower AZ102-EW-T-1 Cooling Fan local control switch HS-AZ102-EWT-1A1 in START.

3.21 VERIFY Evaporator Tower AZ102-EW-T-1 Cooling Fan starts.

Test Engineer:

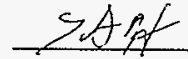

3.22 RECORD the following data for Evaporator Tower AZ102-EW-T-1 Cooling Fan:

3.22.1 Motor Current (TEST INST.) 10.7 Amps

3.22.2 Motor Voltage (TEST INST.) $\frac{490}{401 \text { Volts }}$

Test Engineer:

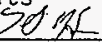

3.23 ALLOW. Evaporator Tower AZ102-EW-T-1 Cooling Fan to operate for one hour for collection of vibration data and bearing data.

3.24 RECORD vibration data and bearing temperatures on Appendix D-4.

Test Engineer:

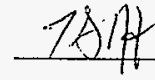

3.25 PLACE Evaporator Tower AZ102-EW-T-1 Cooling Fan remote control switch HS-AZ102-EWT-1 in OFF on graphics screen 06EvpAY1.v.

3.26 VERIFY Evaporator Tower AZ102-EW-T-1 Cooling Fan does NOT STOP..

Test Engineer:

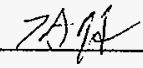

3.27 PLACE Evaporator Tower AZ102-EW-T-1 Pan Heater local control switch HS-AZ102-EWT-1A2 in ON.

3.28 VERIFY Evaporator Tower AZ102-EW-T-1 Pan Heater remains deenergized by checking the heater contactor is OPEN.

Test Engineer:

3.29 SIMULATE a low Evaporator Tower Pan level.

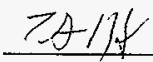




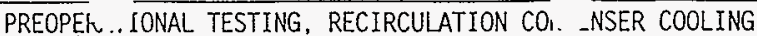
WHC-SD-W030-POTP-003

Revision No. 0

\section{TANK AZ102 RECIRC CONDENSER C}

3.30 VERIFY the following actions occur:

3.30.1 Evaporator Tower Spray Pump AZ102-EW-SP-1 stops.

3.30.2 Evaporator Tower Cooling Fan stops.

3.30.3 Evaporator Tower Makeup Valve EV-AZ102EWT-1A1 opens.

Test Engineer:

3.31 RECORD makeup flow at FI-AZRW-1 (graphics screen 05Water.v). $23 \mathrm{gpm}$.

Test Engineer:

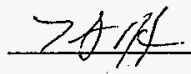

3.32 RESTORE the Evaporator Tower Pan level to normal.

3.33 VERIFY Evaporator Tower Pan Heater energizes by checking the heater contactor is CLOSED.

Test Engineer:

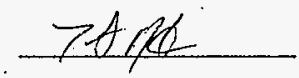

3.34 PLACE Evaporator Tower AZ102-EW-T-1 Temperature Controt Damper local control Switch HS-AZ102-EWT-1A3 in AUTO.

3.35 PLACE Evaporator Tower AZ102-EW-T-1 Spray Pump AZ102-EW-SP-1 Tocal control switch HS-AZ102-EWSP-1A in REMOTE.

3.36 PLACE Evaporator Tower AZ102-EW-T-1 Cooling Fan local control switch HS-AZ102-EWT-1AI in REMOTE.

3.37 PLACE Evaporator Tower AZ102-EW-T-I Spray Pump AZ102-EW-SP-1 remote control switch HS-AZ102-EWSP-1 in START on graphics screen 06EvPAYI.v.

3.38 VERIFY Evaporator Tower AZ102-EW-T-1 Spray Pump AZ102-EW-SP-I starts.

Test Engineer:

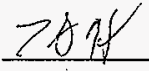

3.39 VERIFY Evaporator Tower Pan Heater deenergizes by checking the heater contactor is OPEN.

Test Engineer:

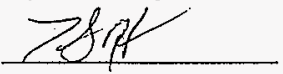

3.40 PLACE Evaporator Tower AZ102-EW-T-1 remote control switch HSAZ102-EWT-1A1 in ON on graphics screen 06EvpAY1.v. 


\section{PREOPERAT.}

Revision No. $\underline{0}$
JAL TESTING, RECIRCULATION CONDL, ER COOLING WHC-SD-W030-POTP-00

ATTACHMENT D
HNF-SD-W030-TD-003, REV. 0, PAGE 102

TANK AZ102 RECIRC CONDENSER

3.41 VERIFY the following events occur:

3.41.1 AZ102 Evaporator Tower Cooling Fan starts.

3.41.2 AZ102 Evaporator Tower AZ102-EW-T-1 Temperature

Control Damper modulates to maintain glycol

temperature at setpoint $100^{\circ} \mathrm{F}$ as indicated on graphics screen06EvpAYl.v.

Test Engineer:

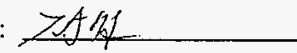

3.42 OPEN Closed Loop Filtration System inlet and outlet block valves HV $-A Z 102 W-1$ and HV-AZ102W-3.

3.43 BUMP the Closed Loop Filtration System Recirc Pump AZ102-W-P-1 by placing control switch HS-AZ102WP-1 in HANO and then back to OFF.

3.44 VERIFY rotation of the Closed Loop Filtration System Recirc Pump AZ102-W-P-1 is in the direction indicated by the arrow on the pump.

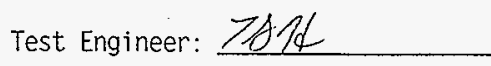

3.45 PLACE the Closed Loop Filtration System Recirc Pump AZ102-W-P-1 local control switch AZ102WP-1 in HAND.

3.46 VERIFY Closed Loop Filtration Recirc Pump AZ102-W-P-I starts.

$$
\text { Test Engineer: ZAf }
$$

3.47 SLOWLY OPEN Closed Loop Filtration System Recirc Pump AZ102-W-P-1 discharge valve HV-AZ102W-2 to establish full system flow.

3.48 RECORD the following data for Closed Loop Filtration Recirc Pump AZ102-W-P-1:

3.48 .1

3.48 .2

3.48 .3

3.48 .4

3.48 .5

Motor Current (TEST INST.) $\frac{2.6}{485}$ Amps
Motor Voltage (TEST INST.)
Recirc Flow (TEST INST.)

Separator Inlet Pressure (LOCAL) 25 psi

Separator Outlet Pressure (LOCAL)

Test Engineer:

$\frac{10}{2 A A P H}$ psi

3.49 ALLOW Closed Loop Filtration Recirc Pump AZ102-W-P-1 to operate for one hour for collection of vibration data and bearing data. 
PREOPER ONAL TESTING, RECIRCULATION CO: WHC.SD-W030-POTP-OI

Revision No. e
ATTACHMENT D

TANK AZ102 RECIRC CONDENSER
NSER COOLING

HNF-SD-W030-TD-003,

REV. 0, PAGE 103

3.50 RECORD vibration data and bearing temperatures on Appendix 0-5.

Test Engineer:

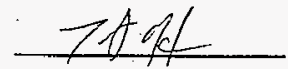

3.51 OPEN Bag Filter AZ102-W-F-2 inlet isolation valve HV-AZ102W-4.

3.52 VENT all air from the filter housing using the pressure/vacuum relief valve on top of the filter.

3.53 OPEN Bag Fj7ter AZ102-W-F-2 outlet jsolation valve HV-AZ102W-5.

3.54 VERIFY bag filter differential pressure indicates in the green range on PDIS-AZ102WF-2.

Test Engineer:

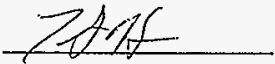

3.55 LOOSEN fitting on Bag Filter PDIS-AZ102WF-2 low side instrument tubing.

3.56 VERIFY Bag Filter high differential pressure alarm PDAH-AZ102WF-2 initiates at 15psid on graphics screen 06EvpAY1.v.

Test Engineer:

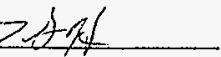

3.57 TIGHTEN fitting Bag Filter PDIS-AZ102WF-2 low side instrument line.

3.58 VERIFY Bag Filter high differential pressure alarm PDAH-AZ102WF-2 clears on graphics screen 06EvpAY1.v.

Test Engineer:

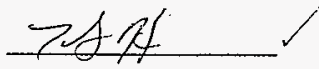

3.59. RECORD the conductivity reading indicated on the Clearview controlier.

3.59.1 Tower fluid conductivity Z40 Micromhos Test Engineer: and

3.60 PLACE the Test Switch on the Clearview Controller in the TEST position.

3.61 VERIFY Evaporator Tower Bleed Valve EV-AZ102EWT-1A2 OPENS.

Test Engineer:

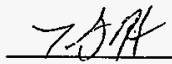


PREOPERAT. ,AL TESTING, RECIRCULATION CONDE, ..ER COOLING WHC-SD-W030-POTP-Or

Revision No. 0 ATTACHMENT D TANK AZ102 RECIRC CONDENSER

HNF-SD-W030-TD-003, REV. 0, PAGE 104

3.62 PLACE the Test switch on the Clearview Controller in the OPER position.

3.63 VERIFY Evaporator Tower Bleed Valve EV-AZ102EWT-1A2 CLOSES.

Test Engineer:

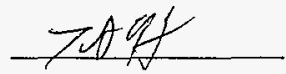

3.64 PLACE the Closed Loop Filtration System Recirc Pump AZ102-W-P-1 local control switch AZ102WP-1 in OFF.

3.65 CLOSE the Closed Loop Filtration System inlet and outlet block valves HV-AZ102W-1 and HV-AZ102W-3.

3.66 IF desired, THEN DRAIN the Closed Loop Filtration System as follows. OTHERWISE continue at step 3.67:

3.66.1 REMOVE drain plug from Closed Loop Filtration System Recirc Pump casing.

3.66.2 DISCONNECT hoses on either side of bag filter.

3.66.3 REMOVE vent plug from top of vent filter.

3.66.4 WHEN system completes draining. THEN reinstall al? plugs and connect hoses to bag filter.

3.67 PLACE Evaporator Tower AZ102-EW-T-1 Spray Pump AZ102-EW-SP-1 remote control switch HS-AZ102-EWSP-1 in STOP.

3.68 VERIFY Evaporator Tower AZ102-EW-T-1 Spray Pump AZ102-EW-SP-1 STOPS.

Test Engineer:

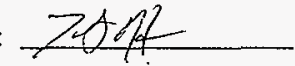

3.69 PLACE Evaporator Tower AZ102-EW-T-1 remote control switch HSAZ102-EWT-1A1 in OFF.

3.70 VERIFY the following events occur:

3.70.1 AZ102 Evaporator Tower Cooling Fan STOPS.

3.70.2 AZ102 Evaporator Tower AZ102-EW-T-1 Temperature Control Damper OPENS.

Test Engineer:

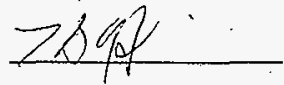

3.71 CLOSE Raw Water supply to the Evaporator Tower HV-AZ102RW-1. 


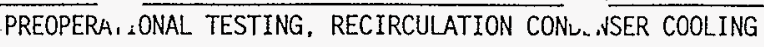
WHC-SD-W030-POTP.

Revision No. 0

ATTACHMENT D

HNF-SD-W030-TD-003,

REV. 0, PAGE 105

TANK AZ102 RECIRC CONDENSE

3.72 IF it is desired to drain the Evaporator Tower. THEN PERFORM the following steps. OTHERWISE continue at step 3.75 .

3.73 PLACE the Test Switch on the Clearview Controlier in the TEST position to allow the Evaporator Tower to drain.

3.74 PLACE the Test Switch on the Clearview Controller in the OPER position.

3.75 VERIFY all test equipment instailed to support this test has been removed.

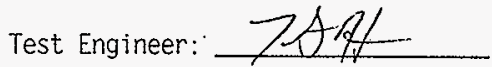


PREOPERAT

Revision No. 0
AL TESTING, RECIRCULATION COND. WHC - SD - WO3O-POTP-OI

ATTACHMENT D

TANK AZ102 RECIRC CONDENSER
ER COOLING

HNF-SD-W030-TD-003,

REV. 0 , PAGE 106

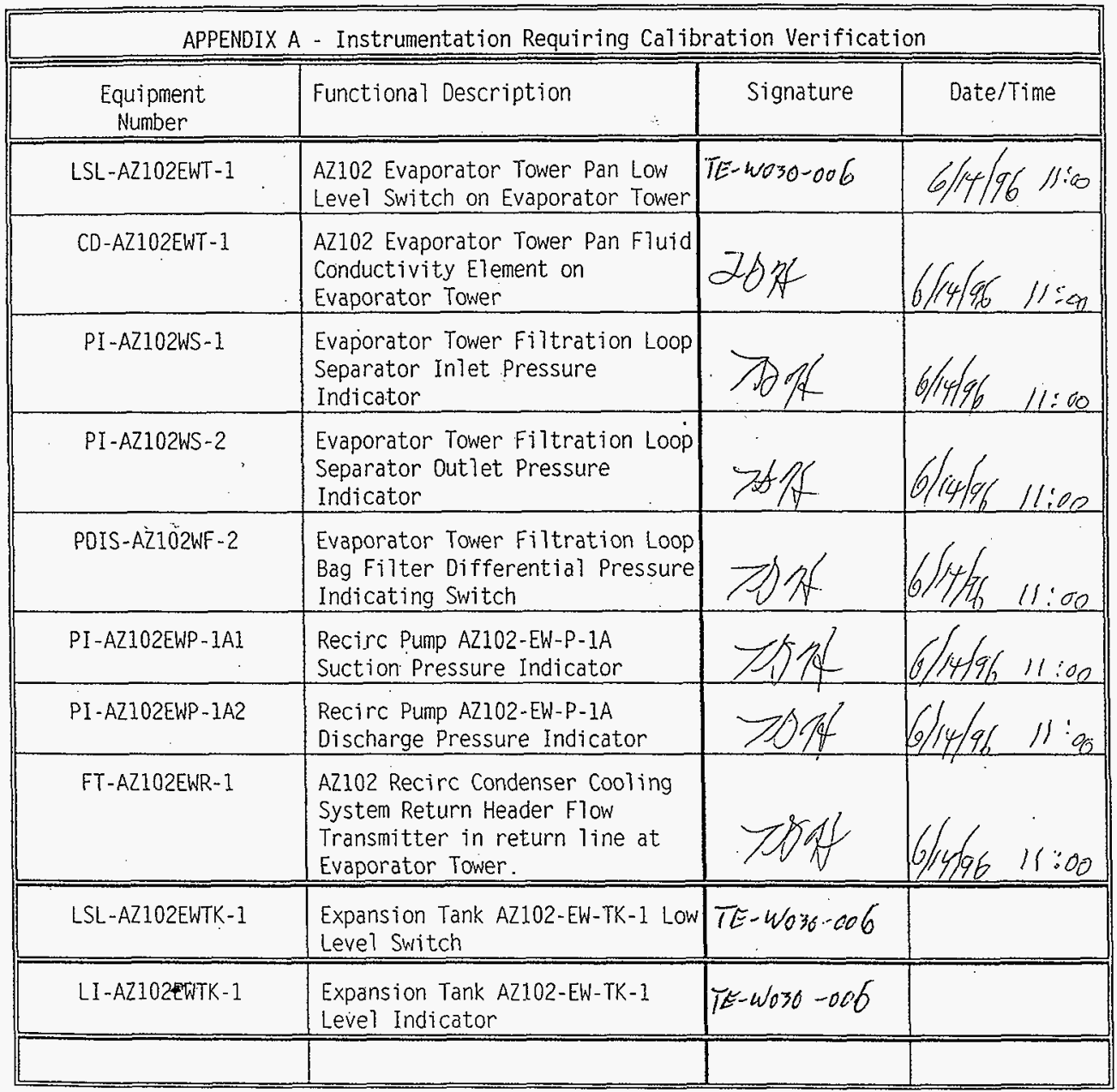


PREOPE. TONAL TESTING, RECIRCULATION CC WHC-SD-W030-POTP-003

Revision No. 0

\section{ATTACHMENT D}

TANK AZ102 RECIRC CONDENSER CO
NSER COOLING

HNF-SD-W030-TD-003, REV. O, PAGE 107

\begin{tabular}{|c|c|c|c|c|}
\hline \multicolumn{5}{|c|}{ APPENDIX B - Tank AZ102 Recirc Condenser Cooling System Valve Alignment } \\
\hline \begin{tabular}{c|} 
VALVE \\
NUMBER \\
\end{tabular} & VALVE NAME & $\begin{array}{l}\text { REQUIRED } \\
\text { POSITION }\end{array}$ & $\begin{array}{c}\text { INITIALS } \\
(1 *) \\
\end{array}$ & $\begin{array}{l}\text { IV } \\
(2)\end{array}$ \\
\hline HV-AZZ102EWP-1A1 & $\begin{array}{l}\text { Recirc Pump AZ102-EW-P-IA } \\
\text { Suction Isolation Valve }\end{array}$ & OPEN & $K_{1} A_{i}$ & \\
\hline HV - AZ102EWP - 1A3 & $\begin{array}{l}\text { Recirc Pump AZ102-EW-P-1A } \\
\text { Suction Pressure Gage Isolation }\end{array}$ & OPEN & $\ell_{1} A$. & \\
\hline HV - AZ102EWP-IA4 & $\begin{array}{l}\text { Recirc Pump AZ102-EW-P-1A } \\
\text { Discharge Pressure Gage Isolation }\end{array}$ & OPEN & $R_{1}, A_{1}$ & \\
\hline HV - AZ102EWP-IA5 & $\begin{array}{l}\text { Recirc Pump AZ102-EW-P.1A } \\
\text { Discharge Isolation }\end{array}$ & CLOSED & $R \cdot A$. & \\
\hline HV-AZ102EWS-1A2 & $\begin{array}{l}\text { Recirc Pump AZ102·EW.P.1A } \\
\text { Discharge Header Isolation }\end{array}$ & OPEN & $R, A$, & \\
\hline HV-AZ102EWP-IBI & $\begin{array}{l}\text { Recirc Pump AZ102-EW-P-1B } \\
\text { Suction Isolation Valve }\end{array}$ & OPEN & $R, f)$ & \\
\hline HV-AZ102EWP-1B3 & $\begin{array}{l}\text { Recirc Pump AZ102-EW-P-1B } \\
\text { Suction Pressure Gage Isolation }\end{array}$ & OPEN & $R_{1} H_{1}$ & \\
\hline HV - AZ102EWP - $1 B 4$ & $\begin{array}{l}\text { Recirc Pump AZ102-EW-P-IB } \\
\text { Discharge Pressure Gage Isolation }\end{array}$ & OPEN & $R . M$. & \\
\hline HV-AZ102EWP-1B5 & $\begin{array}{l}\text { Recirc Pump AZ102-EW-P.IB } \\
\text { Discharge Isolation }\end{array}$ & CLOSED & P.A. & \\
\hline HV-AZ102-EWS-1AI & $\begin{array}{l}\text { Recirc Condenser Cooling System } \\
\text { Supply Header Drain Valve in } 241 \text {. } \\
\text { AY-401 valve box. }\end{array}$ & CLOSED & $R . H$. & \\
\hline HV-AZ102-EWR-IAI & $\begin{array}{l}\text { Recirc Condenser Cooling System } \\
\text { Return Header Drain Valve } 241-A Y \text {. } \\
401 \text { valve box. }\end{array}$ & CLOSED & E.A. & \\
\hline $\begin{array}{l}\text { HV-AZ102-EWTK- } \\
\text { 1A1 } \\
\end{array}$ & $\begin{array}{l}\text { Expansion Tank Level Switch LSL- } \\
\text { AZ102EWTK-1 Low Side Isolation }\end{array}$ & OPEN & P.A. & \\
\hline $\begin{array}{c}\text { HV. AZ102-EWTK- } \\
\text { IA2 }\end{array}$ & $\begin{array}{l}\text { Expansion Tank Level Switch LSL- } \\
\text { AZ102EWTK-1 High Side Isolation }\end{array}$ & OPEN & R.A. & \\
\hline HV-AZ102-EWTK-2 & $\begin{array}{l}\text { Recirc Condenser Cooling System Air } \\
\text { Separator Drain Valve }\end{array}$ & CLOSED & & \\
\hline HV-AZ102-EWT-IAI & Cooling Tower Pan Drain Valve & CLOSED & $R \cdot A$. & $A D Z$ \\
\hline HV-AZ102-RW-1 & $\begin{array}{l}\text { Cooling Tower Raw Water Isolation } \\
\text { at Evaporator Tower. }\end{array}$ & CLOSED & $P A$. & \\
\hline $\mathrm{HV}-\mathrm{AZ10} 2 \mathrm{~W}-1$ & $\begin{array}{l}\text { Closed Loop Filtration System Inlet } \\
\text { Block Valve }\end{array}$ & CLOSED & $R \cdot A$. & \\
\hline
\end{tabular}


PREOPERA1. .NAL TESTING, RECIRCULATION CONDL . .ER COOLING WHC-SD-W030-POTP- 003

Revision No. 2

ATTACHMENT D

HNF-SD-W030-TD-003, REV. O, PAGE 108

\begin{tabular}{|c|c|c|c|c|}
\hline $\begin{array}{l}\text { VALVE } \\
\text { NUMBER }\end{array}$ & VALVE NAME & $\begin{array}{l}\text { REQUIRED } \\
\text { POSITION }\end{array}$ & $\begin{array}{c}\text { INITIALS } \\
(1 *)\end{array}$ & $\begin{array}{l}\text { IV } \\
(2)\end{array}$ \\
\hline $\begin{array}{r}H V \cdot A Z 102 W-2 \\
\not\end{array}$ & $\begin{array}{l}\text { Closed Loop Filtration System } \\
\text { Recirc Pump AZ102-W-P-I Discharge } \\
\text { Valve }\end{array}$ & $\begin{array}{c}\text { CLOSED } \\
\text { से }\end{array}$ & 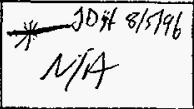 & N/A \\
\hline$H V-A Z 102 W-3$ & $\begin{array}{l}\text { Closed Loop Filtration System } \\
\text { Outlet Block Valve }\end{array}$ & CLOSED & $B, A$. & $21 / 14$ \\
\hline $\mathrm{HV}-\mathrm{AZ102W}-4$ & Bag Filter AZ102-W-F-2 Inlet Valve & CLOSED & & PAft. \\
\hline HV.AZ102W-5 & Bag Filter AZ102-W-F-2 Outlet Valve & CLOSED & $N_{1}$ & 789 \\
\hline HV-AZ102W-6 & $\begin{array}{l}\text { Conductivity Element inlet } \\
\text { isolation valve }\end{array}$ & OPEN & $P_{1} A_{1}$ & HAR \\
\hline HV - AZ102W-7 & $\begin{array}{l}\text { Conductivity Element outlet: } \\
\text { isolation valve }\end{array}$ & OPEN & $\ell_{1} A_{1}$ & ISOA \\
\hline & & & & \\
\hline
\end{tabular}

Performed By

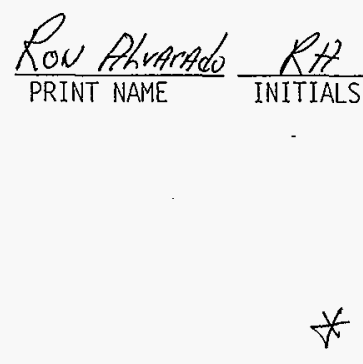

Verified TANK AZ102 RECIRC CONDENSER Cl 
PREOPEF, IONAL TESTING, RECIRCULATION CO WHC-SD-W030-POTP-00:

Revision No. 0 ATTACHMENT D
TANK AZ102 RECIRC CONDENSER C

HNF-SD-W030-TD-003, REV. O, PAGE 109

\begin{tabular}{|c|c|c|c|c|}
\hline $\begin{array}{c}\text { BREAKER } \\
\text { NUMBER }\end{array}$ & BREAKER NAME AND LOCATION & $\begin{array}{l}\text { REQUIRED } \\
\text { POSITION } \\
\end{array}$ & $\begin{array}{c}\text { INITIALS } \\
\left(1^{*}\right)\end{array}$ & $\begin{array}{l}\text { IV } \\
(2) \\
\end{array}$ \\
\hline $1 \mathrm{~B}$ & $\begin{array}{l}\text { Evaporator Tower AZ102 Supply } \\
\text { Breaker in Distribution Panelboard } \\
\text { PP.2 Diesel Generator Building }\end{array}$ & ON & & $78 / 4$ \\
\hline 2 HP SEPARATOR & $\begin{array}{l}\text { Evaporator Tower AZ102 Separator } \\
\text { Recirc Pump Supply Breaker in Local } \\
\text { Panel AZ102-EVAP-TWR }\end{array}$ & OFF & & $7 A \not$ \\
\hline $\begin{array}{l}\text { FLUID COOLER } \\
\text { CONTROL PANEL } \\
\text { AZ102-EW-T-1 } \\
\end{array}$ & $\begin{array}{l}\text { Evaporator Tower AZ102 Fan, Recirc } \\
\text { Pump. Pan Heater Supply Breaker in } \\
\text { Local Panel AZ102-EVAP-TWR }\end{array}$ & OFF & & 21 \\
\hline $\begin{array}{c}\text { RECIRC PUMP } \\
\text { AZ102-EW-P-1A }\end{array}$ & $\begin{array}{l}\text { AZ102 Recirc Condenser Cooling } \\
\text { System Recirc Pump AZ102-EW-P-1A in } \\
\text { Local Panel AZ102-EVAP-TWR }\end{array}$ & OFF & & का \\
\hline $\begin{array}{l}\text { RECIRC PUMP } \\
\text { AZ102-EW-P-1B }\end{array}$ & $\begin{array}{l}\text { AZ102 Recirc Condenser Cooling } \\
\text { System Recirc Pump AZ102-EW-P-IB in } \\
\text { Local Panel AZ102-EVAP-TWR }\end{array}$ & OFF & & ZA 2 \\
\hline PANEL. PP.8 & $\begin{array}{l}\text { Evaporator Tower Panelboard PP-8 } \\
\text { Supply Breaker in Local Panel } \\
\text { AZ102-EVAP.TWR }\end{array}$ & ON & & \\
\hline $\begin{array}{l}\text { Disconnect } \\
\text { switch }\end{array}$ & $\begin{array}{l}\text { Evaporator Tower AZ102 Separator } \\
\text { Recirc Pump local disconnect switch } \\
\text { at pump. }\end{array}$ & OFF & $p$ & 87 \\
\hline & & & & \\
\hline & & & & \\
\hline & & & & \\
\hline & & & & \\
\hline 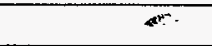 & & & & \\
\hline & & & & \\
\hline
\end{tabular}

Performed

By

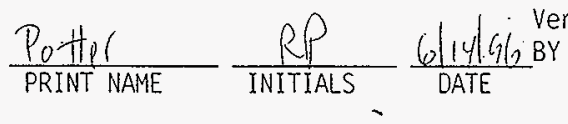

Verified

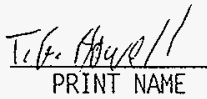

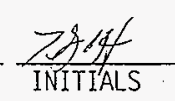

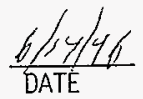




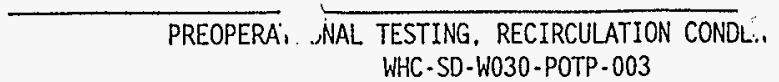

Revision No. $\underline{0}$

ATTACHMENT D

Lucuvive vale

TANK AZ102 RECIRC CONDENSER COOLING SYSTEM

APPENDIX $0-1$

OPERATION DATA SHEET

Equipment Name: TANK AZ102 RECIRC CONOENSER COOLING SYSTEM RECIRC PUMP

EQUIPMENT I.D. NO. : AZ102-EW-P-IA

NAMEPLATE DATA:

See Appendix $-F$ भrc

\begin{tabular}{|l|l|l|l|l|}
\hline \multicolumn{2}{|c|}{ VIBRATION DATA (mi1) } \\
\hline IST READING & $\begin{array}{l}\text { MOTOR } \\
\text { OUTBOARD }\end{array}$ & $\begin{array}{l}\text { MOTOR } \\
\text { INBOARD }\end{array}$ & $\begin{array}{l}\text { PUMP } \\
\text { INBOARD }\end{array}$ & $\begin{array}{l}\text { PUMP } \\
\text { OUTBOARD }\end{array}$ \\
\hline HORIZONTAL & See Test Report, & \\
\hline VERTICAL & Attach ment-3. & \\
\hline AXIAL & Typical all sata sheets \\
\hline
\end{tabular}

\begin{tabular}{|l|l|l|l|l|}
\hline 2ND READING & $\begin{array}{l}\text { MOTOR } \\
\text { OUTBDARD }\end{array}$ & $\begin{array}{l}\text { MOTOR } \\
\text { INBOARD }\end{array}$ & $\begin{array}{l}\text { PUMP } \\
\text { INBOARD }\end{array}$ & $\begin{array}{l}\text { PUMP } \\
\text { OUTBOARD }\end{array}$ \\
\hline \hline HORIZONTAL & & & & \\
\hline VERTICAL & DitO & $\nexists c$ & & \\
\hline AXIAL & & & & \\
\hline
\end{tabular}

\begin{tabular}{|l|l|c|l|c|}
\hline $\begin{array}{l}\text { PUMP BEARING } \\
\text { TEMPERATURES }\end{array}$ & $\begin{array}{l}\text { MOTOR } \\
\text { OUTBOARD }\end{array}$ & $\begin{array}{c}\text { MOTOR } \\
\text { INBOARD }\end{array}$ & $\begin{array}{l}\text { PUMP } \\
\text { OUTBOARD }\end{array}$ & $\begin{array}{c}\text { PUMP INBOARD } \\
\vdots\end{array}$ \\
\cline { 2 - 5 } & & & & $\ldots$ \\
\hline
\end{tabular}

REMARKS:

Performed by :

Date

Verified by : Date 
PREOPER .ONAL TESTING, RECIRCULATION COR. -NSER COOLING

Revision No. 0 WHC-SD.W030-POTP-I

ATTACHMENT D

Wt HNF-SD-W030-TD-003,

REV. 0, PAGE 111

TANK AZ102 RECIRC CONDENSEI

\section{APPENDIX $F$}

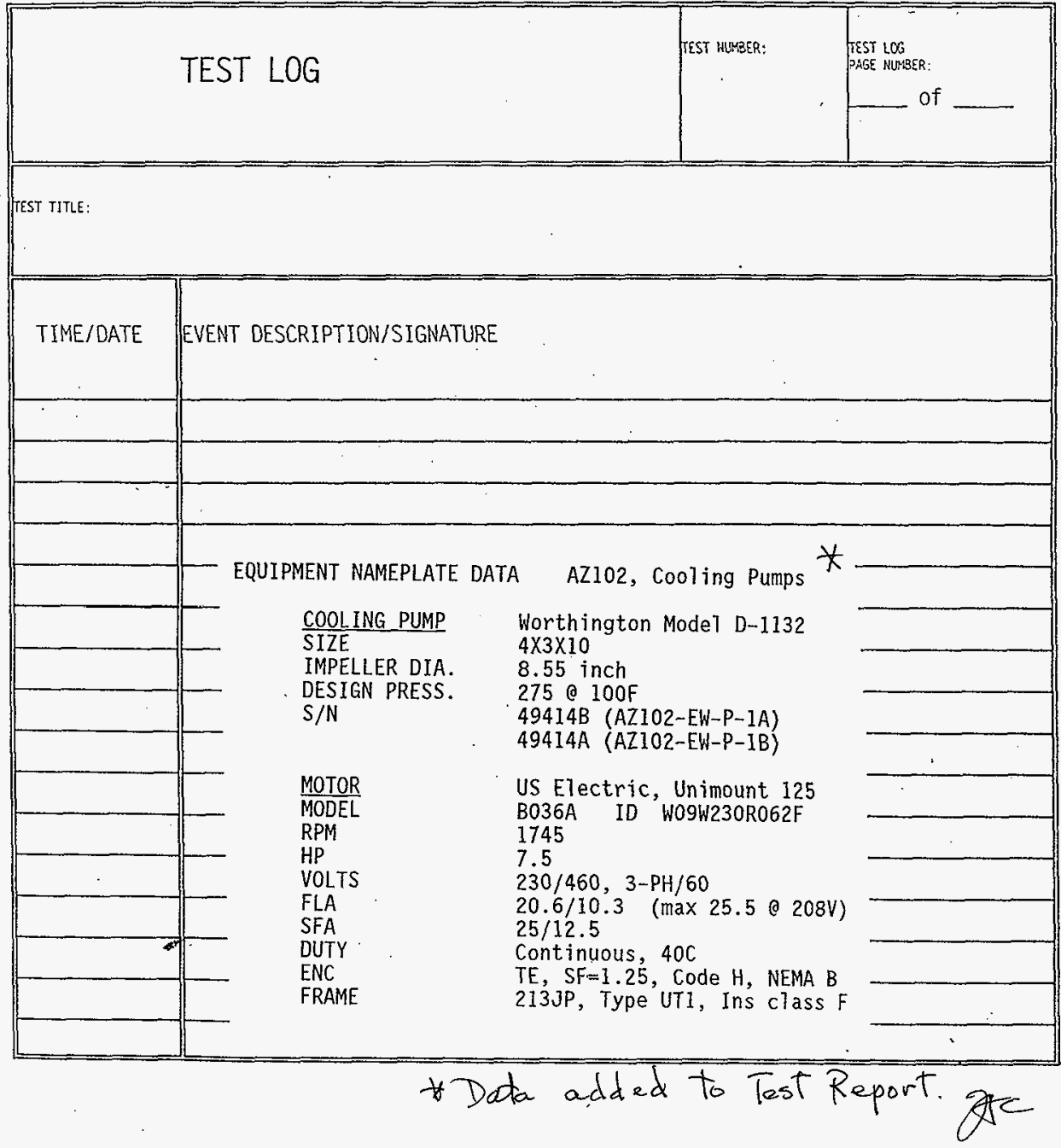


PREOPEK . ONAL TESTING, RECIRCULATION COR. NSFR COOI TNG WHC-SD-W030-POTP-003

Revision No. $\ell$

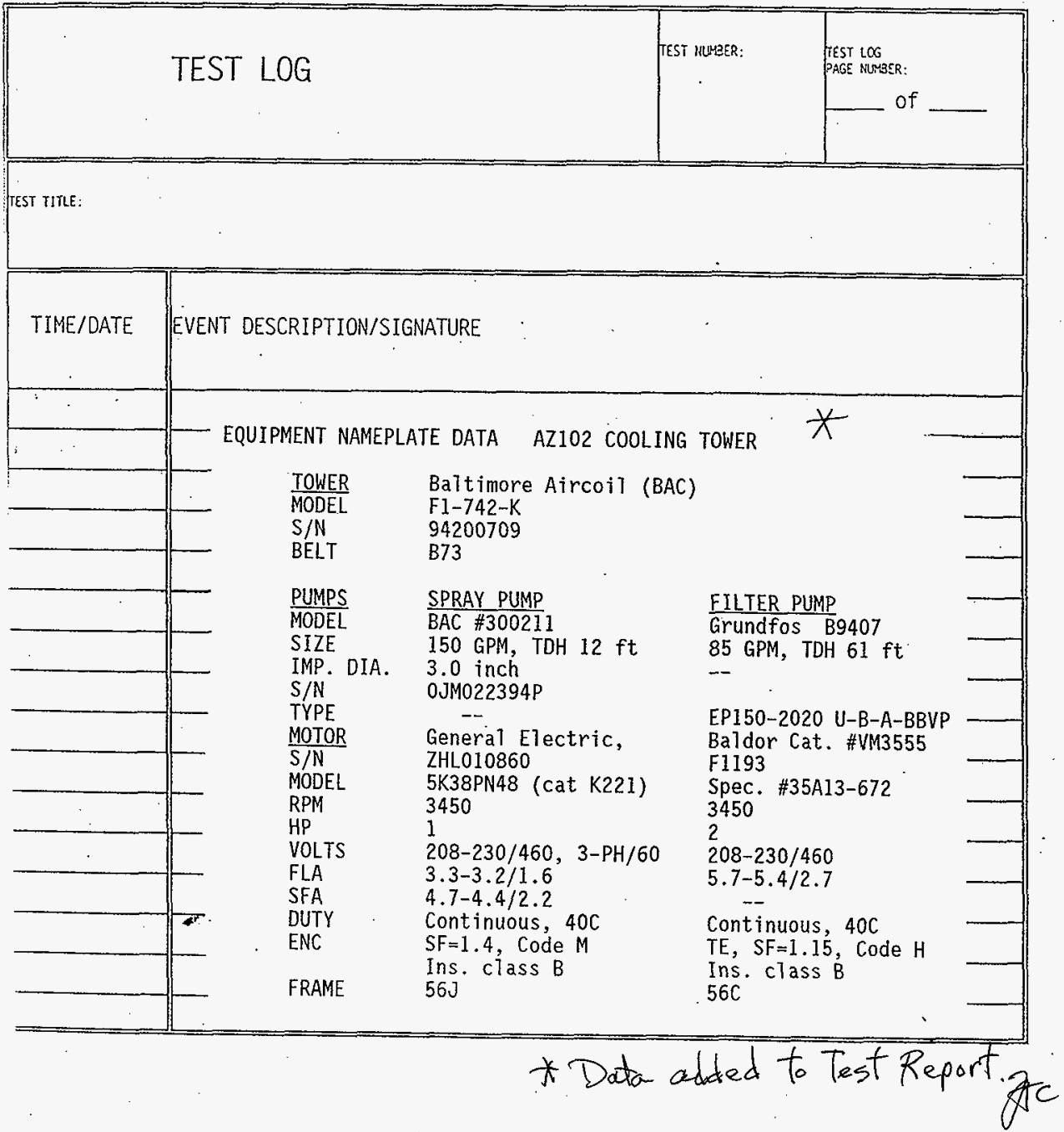


HNF-SD-W030-TD-003,

REV. 0, PAGE 113

\section{ATTACHMENT 3:}

\section{MISCELLANEOUS SUPPORTING DATA}

\section{CERTIFIED VENDOR INFORMATION FILES}

Certified vendor information (CVI) for major equipment is filed as follows:

\begin{tabular}{||l|c|l||}
\hline \multicolumn{1}{|c|}{ EQUIPMENT } & FILE NO. & \multicolumn{1}{|c|}{ VENDOR NAME } \\
\hline \hline Evaporative Cooling Towers & $22525 / 9$ & Baltimore Aircoil \\
\hline Hydronic Components & $22525 / 20$ & Bel1 \& Gossett \\
\hline Centrifugal Pumps & $22525 / 29$ & Worthington \\
\hline
\end{tabular}


PREOPERAT, .. AAL TESTING, RECIRCULATION CONDENSER COOLING
Revision No. 0 WHC-SD-W030-POTP-00
ATTACHMENT B
TANK AYIOZ RECIRC CONDENSER
HNF-SD-W030-TD-003,
REV. 0, PAGE 114

APPENDIX H

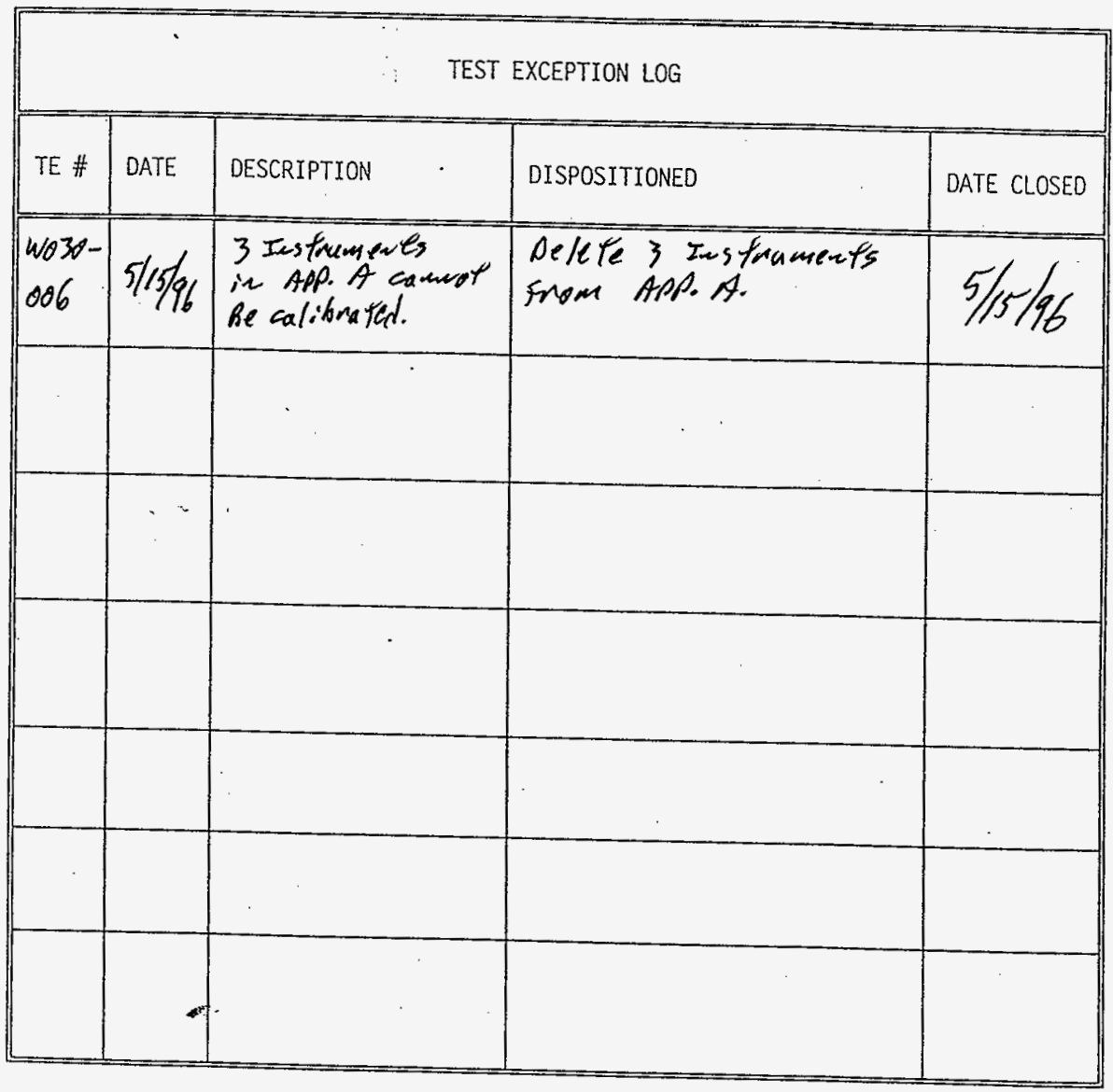


HNF-SD-W030-TD-003,

REV. 0, PAGE 115

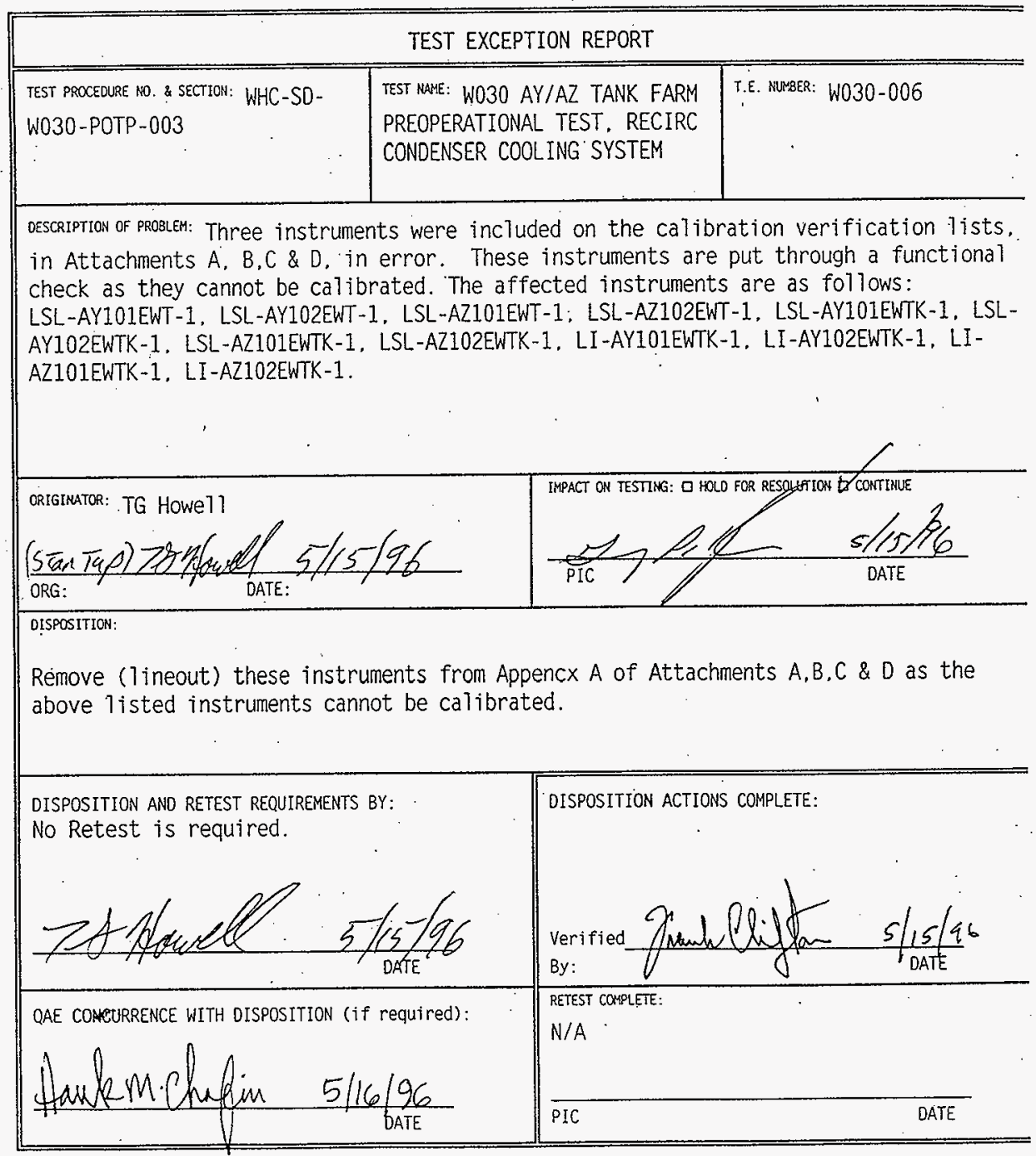




\section{VERIFICATION / VALIDATION CHECKSHEET}

PROCEDURE NUMBER: WHC-SD-WO3O-POTP-003

REVISION NUMBER;

0

PROCEDURE TITLE: Preoperational Testing. Recirculation Condenser Cooling.

\section{VERIFICATION}

PROCEDURE WRITER:

Gary Howe 11

DATE:

DATE: $3 / 11 / 96$

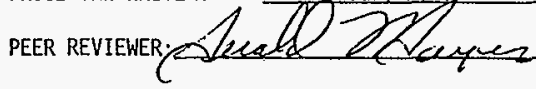

\section{VALIDATION}

OPERATOR: T.K. / Deg DATE: $3 / 12 / 96$

The operator performing the validation must be familiar with area and the systems involved. If there are any No responses, the Operator shal1 document the reason in the DISCREPANCIES Section and ensure that the pertinent information is forwarded to the Procedure Writer for resolution.

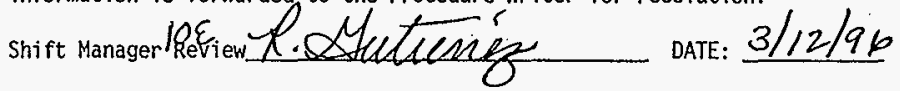

\section{VERIFICATION}

COGNIZANT ENGINEER:

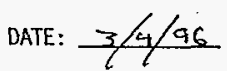

Review validation comments and ensure proper dissposition of validation comments.

Verify that this checksheet can be completed without any NO responses. If there are any NO responses, the justification shall be explained and documented in the DISCREPANCIES Section. 
FROM: M.JENNINGS

TO: F.CLIFTON

DATE: 13 OCT 97

CC; J.COMER, G.HOPKINS, W.MEEUWSEN

SUBJ: W030 COOLING SYSTEM COMPONENT VIBRATION DATA

ALL DATA WAS COLLECTED USING AN SKF, MODEL CMVA10 VIBRATYON ANALYZER/DATA COLLECTOR, HANFORD CALIBRATION NUMBER 752-84-02001, CALIBRATION DUE DATE 5 DEC 97.

THE VALUES ASSOCIATED WITH THE LISTED COMPONENTS ARE RECORDED AS A "PEAK VELOCITY" IN INCHES PER SECOND (IPS) THE OVERALL (OA) VALUE SHOWS THE MEAN OF THE ENTIRE VIBRATION SPECTRUM FROM O TO 12000 CYCLES PER MINUET (CPM). THE X1 VALUES INDICATE THE SPECTRAL PEAK AT 1 TIMES THE EQUIPMENTS RUN SPEED. X1 IS ALSO REFERRED TO AS THE EQUIPMENTS FUNDAMENTAL FREQUENCY. READINGS WERE TAKEN IN THE HORIZONTAL PLAIN ONLY.

$$
\text { AY101-W-P-1 }
$$

1 HORIZ

2 HORIZ

.161 .117

OA, .139 X1

$\mathrm{OA}, .101 \mathrm{X1}$

$$
\text { AZ101-W-P-1 }
$$

1 HORIZ .155 OA, .125 X1

2 HORIZ .108 OA, $.090 \mathrm{XI}$

$$
\mathrm{AY} 101-\mathrm{EW}-\mathrm{SP}-1
$$

1 HORIZ

2 HORIZ $.240 \mathrm{OA}, .237 \mathrm{X} 1$

$$
\text { **AZ101-EW-SP-1** }
$$

1 HORIZ $.046 \mathrm{OA}, .019 \mathrm{X} 1$

2 HORIZ .032 OA, .019 X1

$$
\text { AY 102 -W-P-1 }
$$

1 HORIZ .182 OA, .151 X1

2 HORIZ .164 OA, .145 X1

$$
\text { AZ102-W-P-1 }
$$

1 HORIZ .175 OA, .150 X1

2 HORIZ .216 OA, .206 X1

$$
\text { AY102-EW-SP-1 }
$$

1 HORIZ .124 OA, .118 X1

2 HORIZ .114 OA, .111 X1

$$
\text { AZ102-EW-SP-1 }
$$

1 HORIZ .236 OA, .229 X1

2 HORIZ .127 OA, .124 XI

AZ SPRAY PUMP MARKED "**" HAS A BAD UPPER BEARING.

$$
\text { AY101-EW-P-1A }
$$

1 HORIZ

2 HORIZ

$$
\begin{array}{lll}
.054 & \mathrm{OA}, & .032 \times 1 \\
.030 & \mathrm{OA}, & .013 \mathrm{X1}
\end{array}
$$

AY101-EW-P-1B

1 HORIZ .204.OA, .203 X1

2 HORIZ .138 OA, $.137 \mathrm{X} 1$ 


$$
\text { AY } 102-E W-P-1 A
$$

$\begin{array}{llll}1 & \text { HORIZ PUMP MISSING } \\ 2 & \text { HORIZ } & \text { " }\end{array}$

AY CIRC PUMP MARKED "**" HAS A SERIOUS UNBALANCE.

$$
\text { AZ101-EW-P-1A }
$$

1 HORIZ

$$
.110 \mathrm{OA}, .096 \mathrm{X1}
$$

2 HORIZ .043 OA, .030 X1

$$
\text { AZ102-EW-P-1A }
$$

1 HORIZ .032 OA, .005 X1

2 HORIZ $.023 \mathrm{OA}, .005 \mathrm{X1}$

$$
\mathrm{A} Z-\mathrm{CW}-\mathrm{p}-1 \mathrm{~A}
$$

1 HORIZ

2 HORIZ " "

AZ102-EW-P-1B

$\mathrm{AZ}-\mathrm{CW}-\mathrm{P}-1 \mathrm{~B}$
**AY102-EW-P-1B**

1 HORIZ .793 OA, .737 X1

2 HORIZ .532 OA, .519 X1

1 HORIZ .195 OA, $.176 \mathrm{X1}$

2 HORIZ .108 OA, $.097 \times 1$

$\begin{array}{llll}1 & \text { HORIZ } & \text { PUMP } & \text { MISSING } \\ 2 & \text { HORIZ } & \text { " }\end{array}$

1 HORIZ .053 OA, .048 X1

2 HORIZ .028 OA, .025 X1

1. ALI VALUES - IPS PEAK VELOCITY

2. BEARING DESIGNATOR;

1 INDICATES MOTOR SHAFT FREE END BEARING.

2 INDICATES MOTOR SHAFT COUPLING END BEARING.

3 INDICATES FAN SHAFT COUPLING END BEARING.

4 INDICATES FAN SHAFT FAN END BEARING.

THE ABOVE VALUES WERE OBTAINED ON 13 JULY 97. THESE READINGS WERE TAKEN AS A FOLLOW UP TO THE ORIGINAL MEMO OF 4 AUG 96 AND WERE TAKEN TO ESTABLISH A QUICK CHECK OF EQUIPMENT CONDITION PRIOR TO SERVICE.

THE INSTALLATION PROBLEMS ASSOCIATED WITH THE CLOSED LOOP FILTER PUMPS HAS BEEN CORRECTED AND THE VIBRATION READINGS REFLECT THIS.

WITH THE EXCEPTION OF THE FOLLOWING TWO ISSUES, THE ABOVE VALUES INDICATED A GENERALLY ACCEPTABLE CONDITION FOR THIS TYPE OF EQUIPMENT.

1. THE 101-AZ COOLING TOWER SPRAY PUMP HAS A BAD UPPER BEARING. THE PUMP/MOTOR ASSEMBLY REQUIRES REPLACEMENT PRIOR TO PLACING THE SYSTEM IN A FULLY OPERATIONAL STATUS.

2. THE CONSISTENCY OF RECORDED VALUES FOR SIMILAR EQUIPMENT TYPES, SPECIFICALLY THE COOLING WATER CIRC PUMPS ( $* * * * *-E W-$ P-1A AND B). THESE READING RANGE FROM A LOW OF .023 IPS, 
PEAK, TO A HIGH OF .793 IPS, PEAK. BECAUSE THE PUMP AND MOTOR MANUFACTURERS ARE THE SAME, ONE WOULD THINK THAT THE READINGS SHOULD BE CONSISTENT. BECAUSE OF THE VERTICAL MOUNTING POSITION, ANY UNBALANCE WILL RESULT IN AMPLITUDE CHANGES OF THIS NATURE. THE AY102-EW-P-1B SHOULD BE TAKEN OUT OF SERVICE AS SOON AS PRACTICAL, NOT TO INTERFERE WITH ANY REMAINING TESTING. AT .793 IPS, THIS PUMP WILL NOT LAST VERY LONG. THIS PUMP IS NOT ACCEPTABLE FOR TURNOVER TO OPERATIONS.

\section{RECOMMENDATIONS (SPECIFIC TO AY102-EW-P-1B)}

1. CHECK ALL MOUNTING FASTENERS TIGHT, SPECIFICALLY THE CONCRETE MOUNTING ANCHORS.

2. SWAP THIS PUMPS MOTOR WITH ONE OF THE SMOOTHER PUMPS. IF IT IS A MOTOR BALANCE PROBLEM, THE HIGH LEVELS SHOULD FOLLOW. IF IT IS SHOWN TO BE A BALANCE PROBLEM, LOOKING INTO PROCURING A BETTER QUALITY MOTOR FOR THIS APPLICATION IS RECOMMENDED.

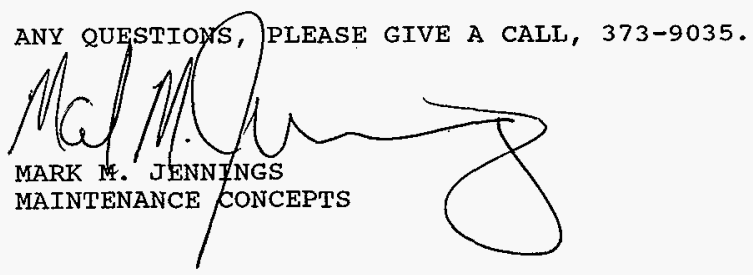


FROM: M.JENNINGS

TO: G. HOWE

DATE: 4 AUG 96

SUBJ: WO30 COOLING.SYSTEM COMPONENT VIBRATION DATA.

ALL DATA WAS COLLECTED USING A SKF, MODEL CMVA10 VIBRATION ANALYZER/DATA COLLECTOR, HANFORD CALIBRATION NUMBER 752-84-02-001, CALIBRATION DUE DATE 6 NOV 96.

THE FOLLOWNG VALUES ASSOCIATED WITH THE FOLLOWING COMPONENTS ARE LISTED AS "PEAK VELOCITY" (INCHES PER SECOND, IPS) WITH THE OVERALL (OA) VALUE SHOWING THE MEAN OF THE ENTIRE SPECTRUM FROM 0 TO 12000 CYCLES PER MINUET (CPM). THE TIMES ONE (X1) REPRESENTS THE EQUIPMENTS FUNDAMENTAL FREQUENCY (EQUIPMENTS RUN SPEED) SPECTRAL PEAK. ALL. READINGS WERE TAKEN IN THE HORIZONTAL PLAIN TO ESTABLISH A QUICK CHECK OF EQUIPMENT CONDITION PRIOR TO SERVICE.

102-AY

EVAPORATOR TOWER SPRAY PUMP, AY102-EW-SP-1

MOTOR OUTBOARD - .25 OA, $.13 \times 1$ MOTOR INBOARD - .22 OA, $.19 \times 1$

EVAPORATOR TOWER COOLING FAN

MOTOR OUTBOARD - .612 OA, .093 X1

FAN CENTER - .166 OA, $.03 \times 1$

FAN INBOARD - .106 OA, $.06 \times 1$

EVAPORATOR TOWER CLOSED LOOP FILTER PUMP, AY102-W-P-1

MOTOR OUTBOARD - .39 OA, $.37 \times 1$ MOTOR INBOARD - .52 OA, $.51 \times 1$

CONDENSER COOLING SYSTEM RECIRC PUMP, AY102-EW-P-1A.

MOTOR OUTBOARD - .49 OA, $.47 \times 1$ MOTOR INBOARD - .4 OA, $.39 \times 1$

CONDENSER COOLING SYSTEM RECIRC PUMP, AY102-EW-P-1B

MOTOR OUTBOARD - .17 OA, $.16 \times 1$ MOTOR INBOARD - .27 OA, $.26 \times 1$

A01-AY

EVAPORATOR TOWER SPRAY PUMP, AY101-EW-SP-1

MOTOR OUTBOARD - .255 OA, $.23 \times 1$ MOTOR INBOARD - .19 OA, $.17 \times 1$ 
EVAPORATOR TOWER COOLING FAN

MOTOR OUTBOARD - .602 OA, $.03 \times 1$

FAN CENTER - .161 OA $.023 \times 1$

FAN INBOARD - .117 OA, $.03 \times 1$

FAN OUTBOARD - .093 OA, $.027 \times 1$

EVAPORATOR TOWER CLOSED LOOP FILTER PUMP, AY101-W-P-1

MOTOR OUTBOARD - .104 OA, $.06 \times 1$ MOTOR INBOARD - 13 OA, $12 \times 1$

CONDENSER COOLING SYSTEM RECIRC PUMP, AY101-EW-P-1A

PUMP NOT OPERATIONAL.

CONDENSER COOLING SYSTEM RECIRC PUMP, AY101-EW-P-1B

PUMP NOT OPERATIONAL.

102-AZ

EVAPORATOR TOWER SPRAY PUMP, AZ102-EW-SP-1

MOTOR OUTBOARD - .205 OA, $.157 \times 1$ MOTOR INBOARD - .13 OA, $.07 \times 1$

EVAPORATOR TOWER COOLING FAN

MOTOR OUTBOARD - .595 OA, $.025 \times 1$

FAN CENTER - .13 OA, $.003 \times 1$

FAN INBOARD - .114 OA, $.03 \times 1$

EVAPORATOR TOWER CLOSED LOOP FILTER PUMP, AZ102-W-P-1

MOTOR OUTBOARD - .16 OA, $.13 \times 1$ MOTOR INBOARD - .29 OA, $.27 \times 1$

CONDENSER COOLING SYSTEM RECIRC PUMP, AZ102-EW-P-1A

MOTOR OUTBOARD - .05 OA, N/AX1 MOTOR INBOARD - .09 OA, N/A X1

CONDENSER COOLING SYSTEM RECIRC PUMP, AZ102-EW-P-1B

MOTOR OUTBOARD - .07 OA, N/AX1 MOTOR INBOARD - .04 OA, N/A X1

101-AZ

EVAPORATOR TOWER SPRAY PUMP, AZ101-EW-SP-1

MOTOR OUTBOARD - .124 OA, .002 X1 MOTOR INBOARD - .18 OA, 1 X1 
EVAPORATOR TOWER COOLING FAN

MOTOR OUTBOARD - .162 OA, $.01 \times 1$

FAN CENTER - .166 OA, N/A X1

FAN INBOARD - .133 OA, N/AX1

FAN OUTBOARD - .147 OA, N/A X1

EVAPORATOR TOWER CLOSED LOOP FILTER PUMP, AZ101-W-P-1

MOTOR OUTBOARD - .172 OA, .01 X1 MOTOR INBOARD - 13 OA, $.1 \times 1$

CONDENSER COOLING SYSTEM RECIRC PUMP, AZ101-EW-P-1A

PUMP NOT OPERATIONAL.

CONDENSER COOLING SYSTEM RECIRC PUMP, AZ101-EW-P-1B

PUMP NOT OPERATIONAL.

A NUMBER OF THE ABOVE READINGS, SPECIFICALLY ON THE COOLING TOWER FAN MOTORS, HAVE AMPLITUDES THAT EXCEED .5 IPS OA WTH THE X1 LEVELS AT LESS THEN $50 \%$ OF THE OVERALL. THIS INDICATES A PROBLEM ASSOCIATED WITH MOTOR MOUNTING STRUCTURE. AT THESE AMPLITUDES, THE MOUNTING STRUCTURE WILL FATIGUE AND FAIL PROBABLY WITHIN THE FIRST FEW YEARS OF OPERATION. THIS IS NOT A 20 YEAR SERVICE LIFE PIECE OF EQUIPMENT AND WLL CREATE A CHRONIC MAINTENANCE CONCERN.

THE CLOSED LOOP FILTER PUMP ASSOCIATED WITH THE 102-AY COOLING TOWER EXHIBIT FAIRLY HIGH READINGS. AGAIN, THIS RELATES TO MOUNTING PROBLEMS. VISUAL INSPECTION SHOWS 3 OUT OF 4 OF THESE PUMPS ARE INCORRECTLY INSTALLED. THE MOUNTING FASTENERS ARE MISSING OR NOT INSTALLED AND THE PUMP DOES NOT SIT CORRECTLY IN ITS MOUNTING SADDLE. IF NOT CORRECTED THIS WILL PROBABLY INITIATE A CHRONIC MAINTENANCE ISSUE.

THE 1A CONDENSER COOLING WATER RECIRC PUMP ASSOCIATED WITH 102-AY HAS ALSO EXHIBITS A HIGH READING WITH THE X1 VALUE DOMINANT. THIS IS AN INDICATION OF AN UNBALANCE. AGAIN, IF NOT CORRECTED THIS WILL PROBABLY INITIATE A CHRONIC MAINTENANCE ISSUE.

IF YOU WOUL LIKE SPECIFIC RECOMMENDATIONS ON CORRECTIVE ACTIONS THAT HAYE A PRQY YEN FRACK RECORD IN EAST TANK FARMS, PLEASE GIVE A CALL, 373-9035.

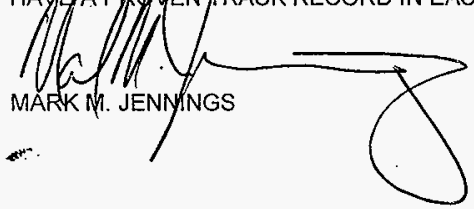




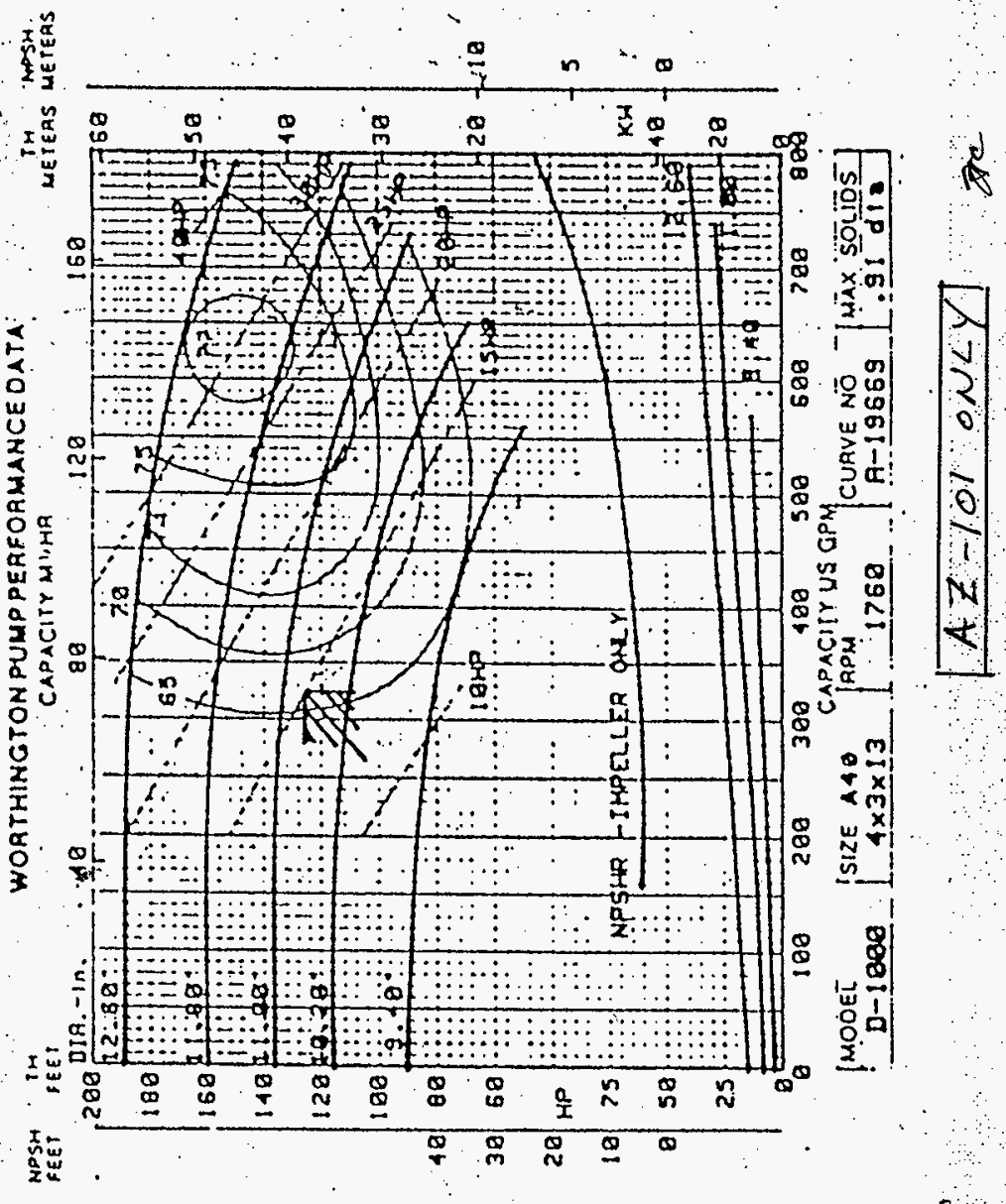




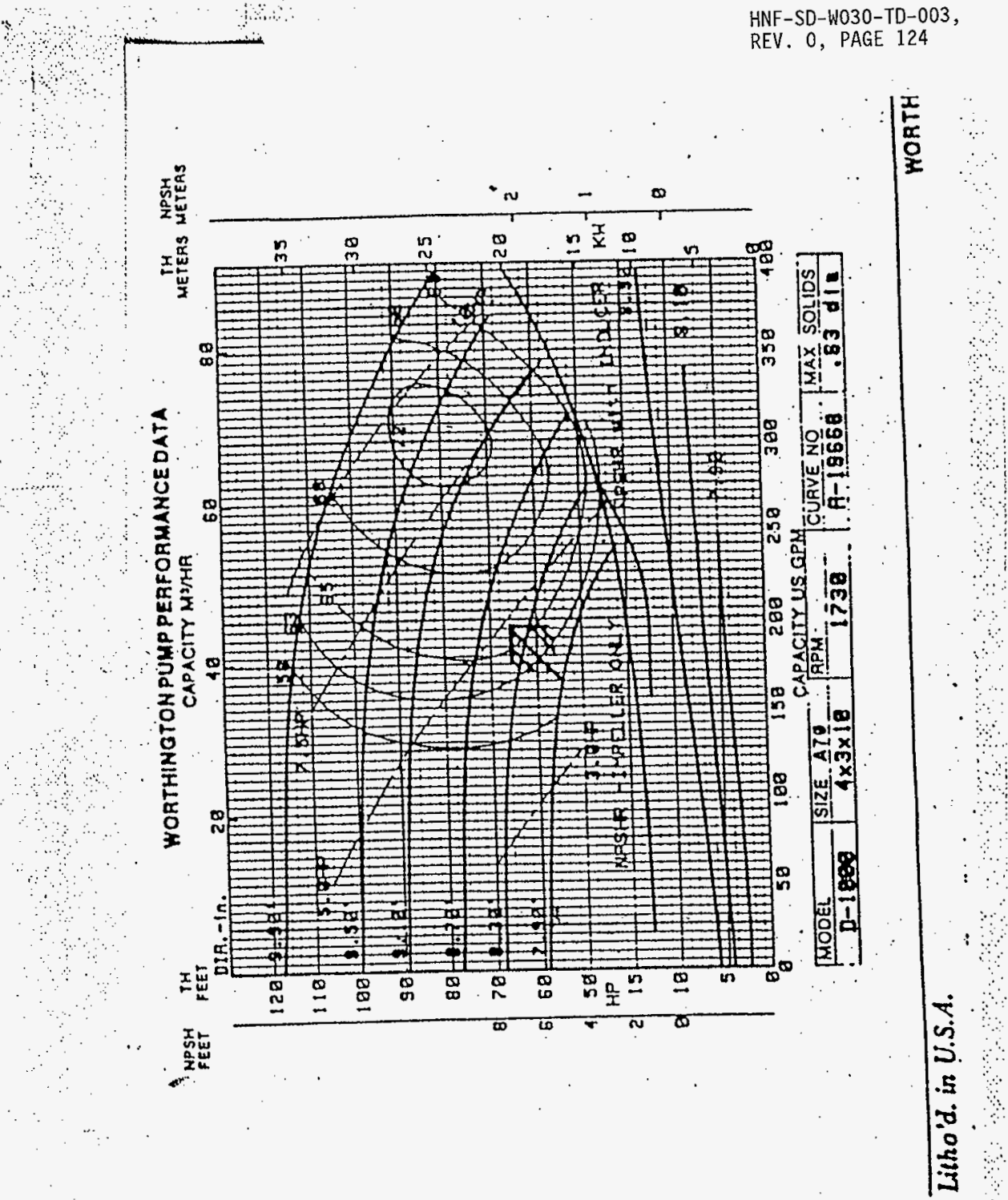


HNF-SD-W030-TD-003,

REV. 0, PAGE 125
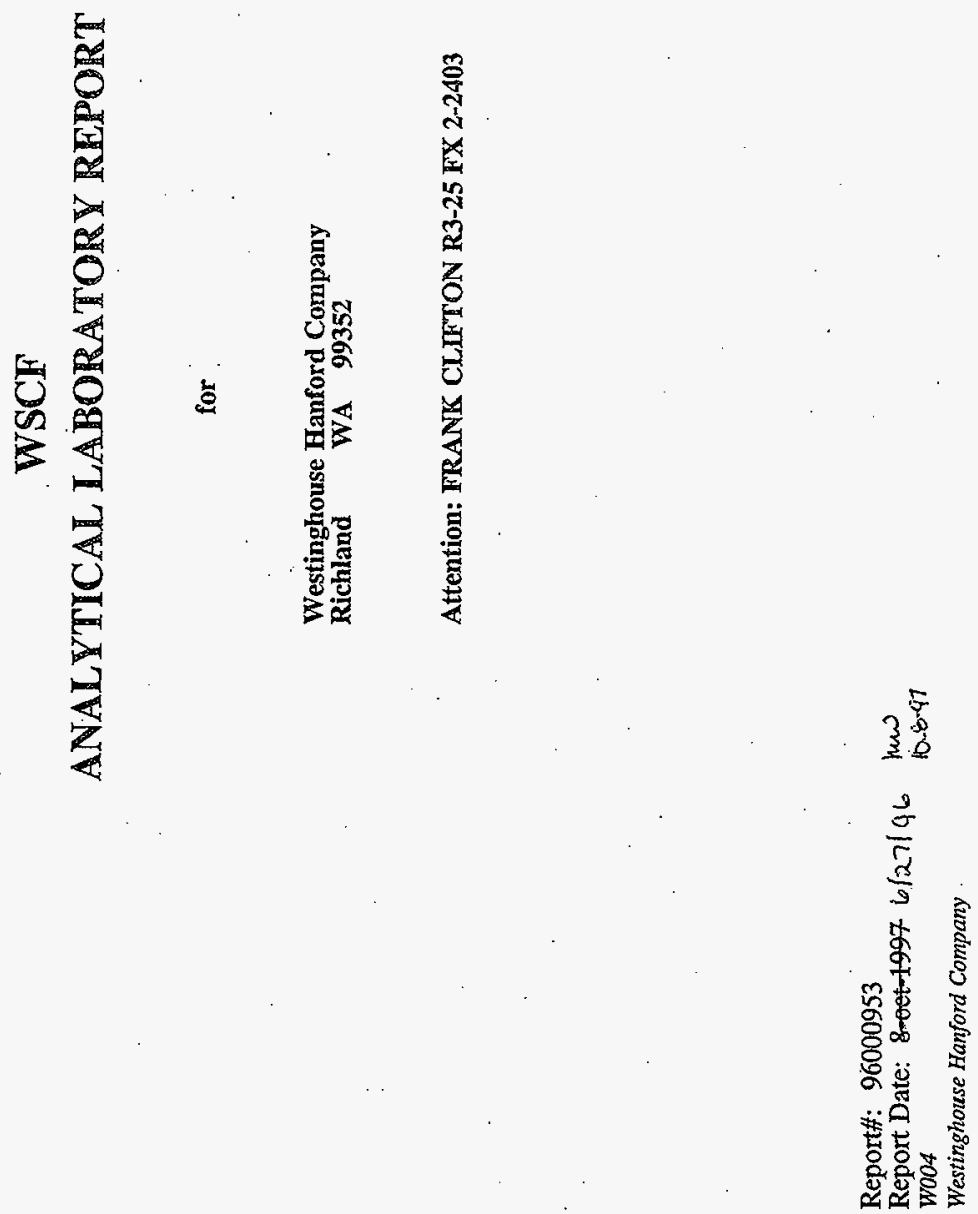


\section{Aftention: \\ FRANK CLIFTON R3-25 FX 2-2403 \\ Project Number. \\ MISC. : :MISCELLANEOUS WHC PROJECTS}

Group \#: 96000730

\begin{tabular}{|c|c|c|c|c|c|c|c|c|c|c|}
\hline Sample \# & Client D & Test Performed & Matrix & Method & RQ & Result & Units & $\mathrm{MOL}$ & Sampled & ReceiYed \\
\hline$\overline{\text { W900001792 }}$ & AY 101 & Miscollanteous Organic Analysoz & WATER & Best Availab & & $49 \% P G$ & & 0.000 & $05117 / 96$ & $05 / 17 / 96$ \\
\hline
\end{tabular}

MDL $\approx$ Minimum Detection Limit

$R Q=$ Result Qualifier $\quad B$ - The analyte was detocted in the associated mothod blank.

$N$ - Identification lis basod on a mass spectra! library sojrch.

- indicates resulte that hava NOT boen validated.

Westinghouse Hanford Company
J - Estimated value.

$U$ - The analyte was analyzed for but not detected. 


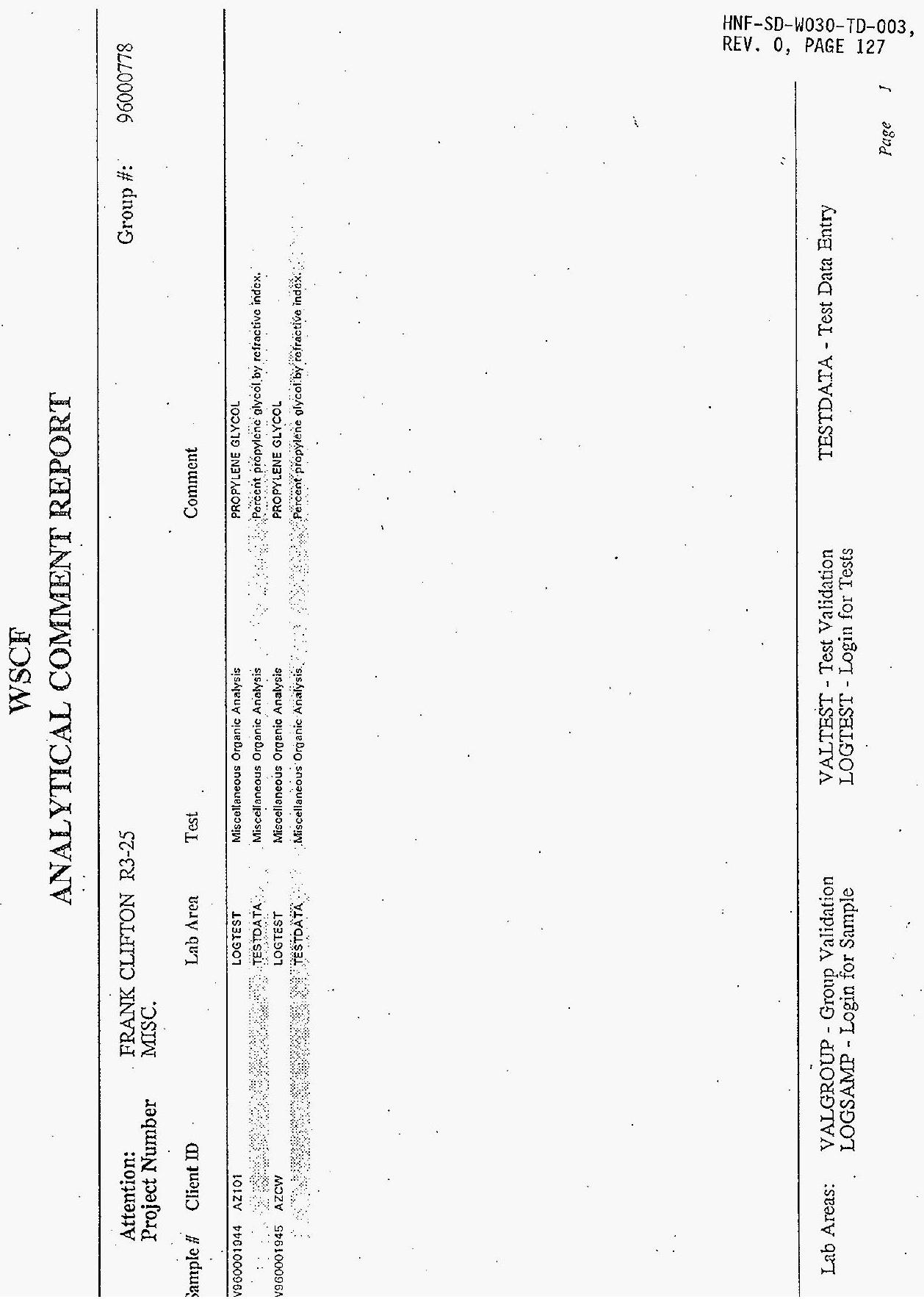




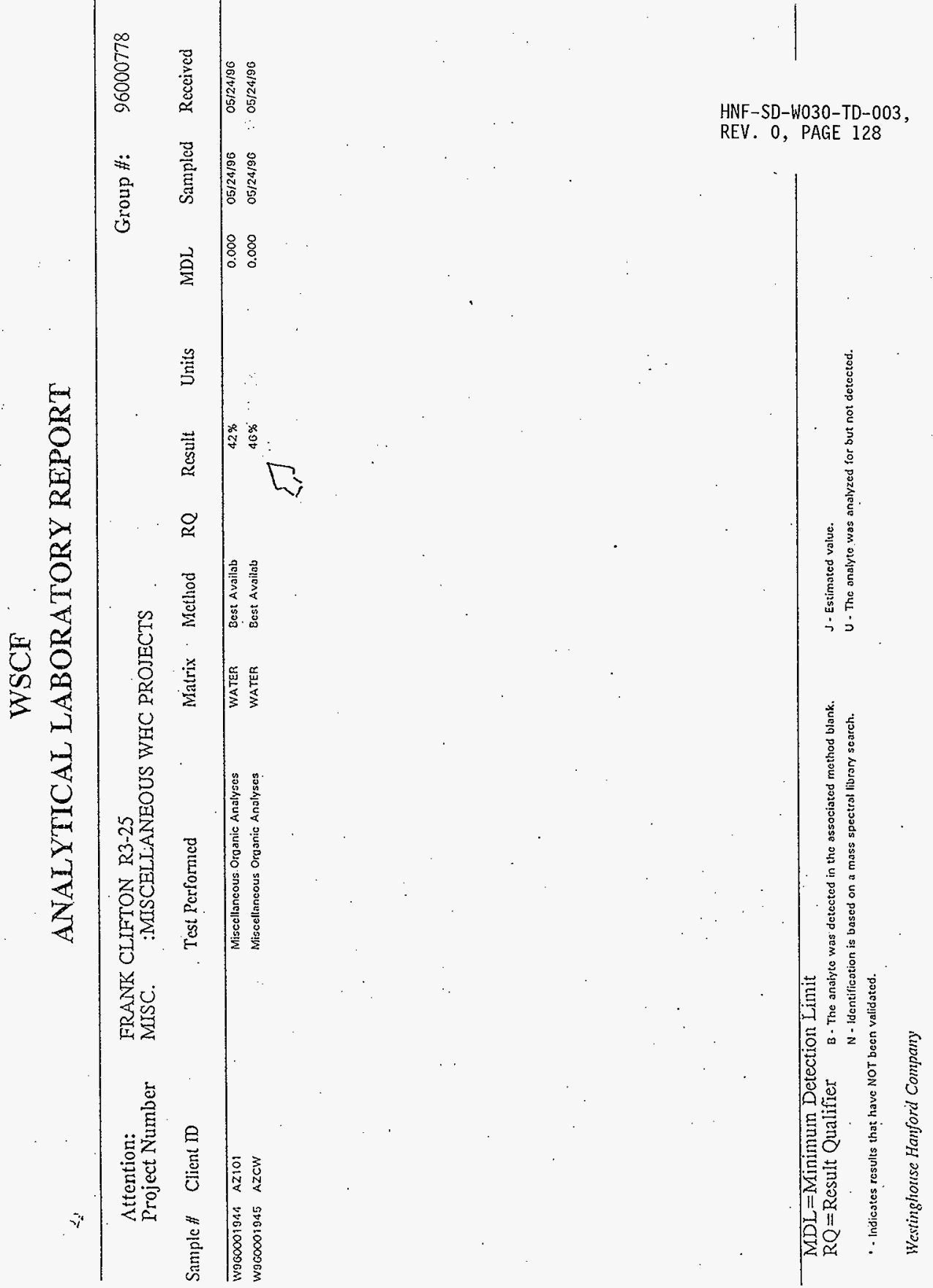




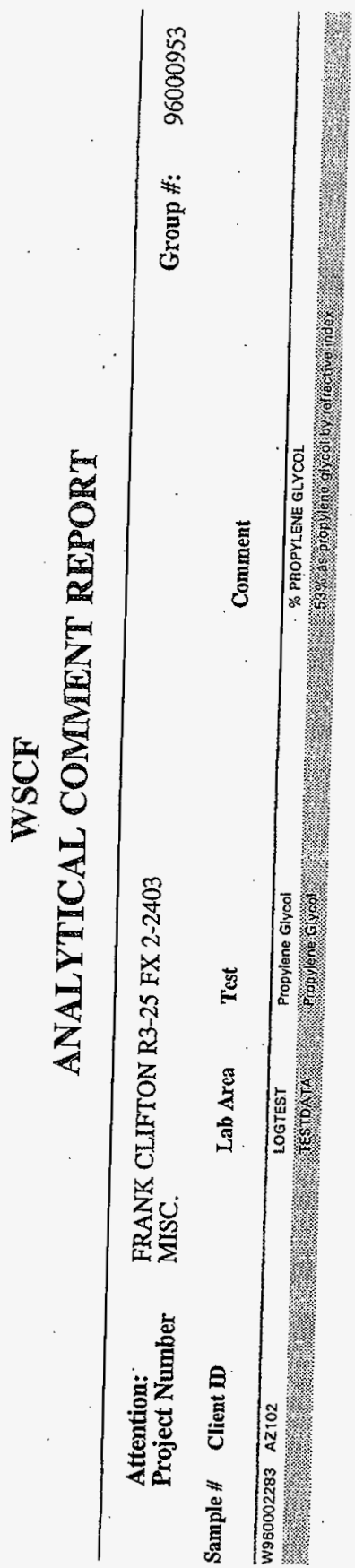

HNF-SD-W030-TD-003, REV. O, PAGE 129

$\stackrel{\Xi}{\infty}$

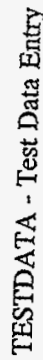

总

훙

速

$\leftrightarrow$,

11

$\forall \in$ 응

본도

방

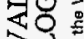

$>-1$

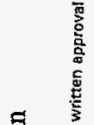

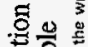

害甭

ส๊

운하 :

옵.

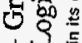

1?

sis:

造

$>0$

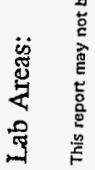




\section{WSCF}

\section{ANALYTICAL LABORATORY REPORT}

Attention: Project Number
FRANK CLIFTON R3-25 FX 2-2403

MISC. :MISCELLANEOUS WHC PROJECTS
Group \#: 96000953

Test Perfined
Matrix Method RQ

WATER ASTM

Result
MOL

Mer
Sampled . Received

\section{MDL $=$ Minimura Detection Limit}

$\mathrm{RQ}=$ Result Qualifier

B - The analyte was detected in the associated method blank.

E - Compound concentration exceeded calibration range.

$\mathrm{N}$ - tdentification is based on a mass spectral library search.

" - Indicates results that have NOT been valifated.

W004

Westinghouse Hanford Company
D. Compound concentration resulted from a dilution.

J. Estimated value. $z$ - see comments.

$U$. The analyte was analyzed for but not detected. 
HNF-SD-W030-TD-003,

REV. 0, PAGE 131

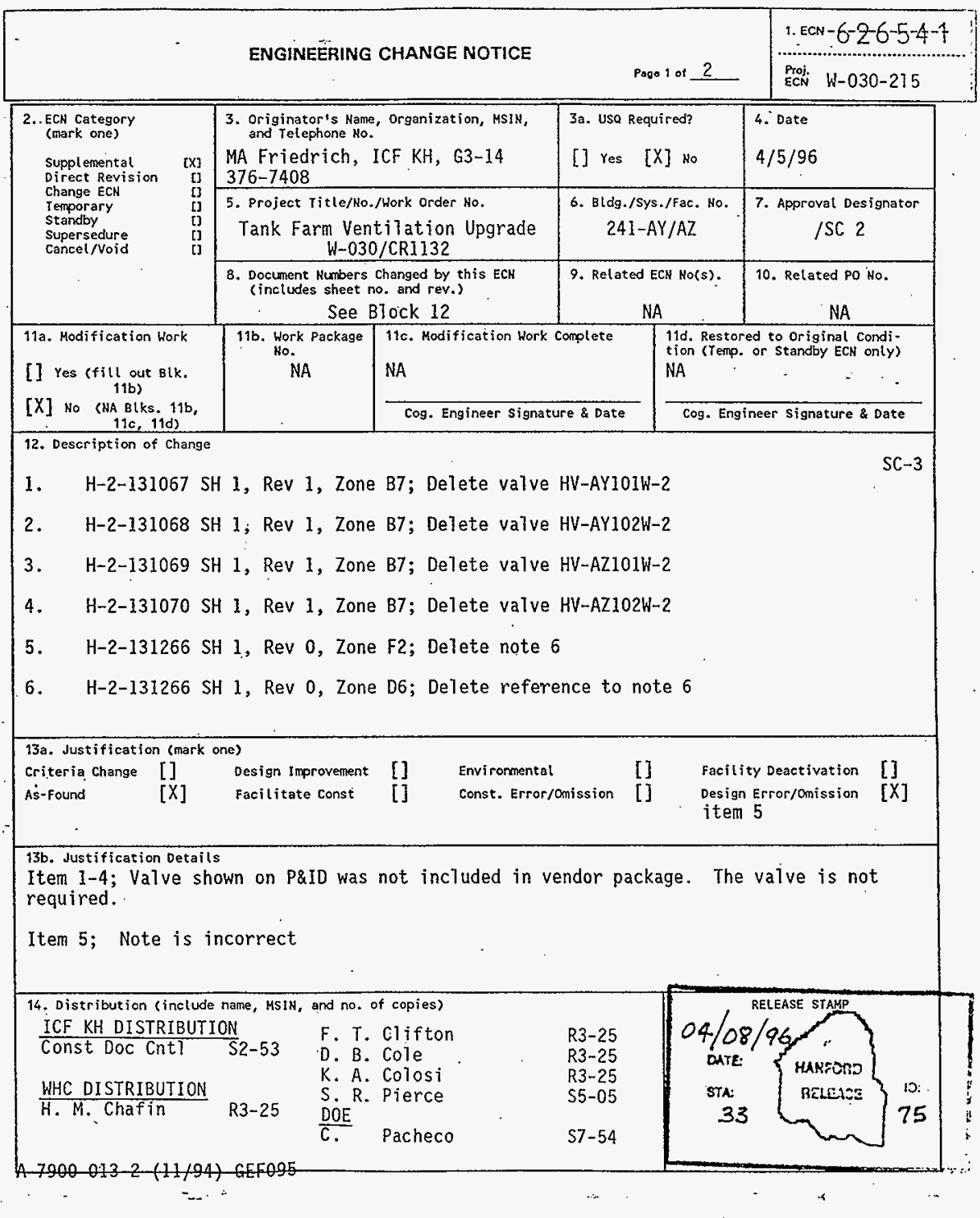




\section{ATTACHMENT 4}

\section{CALIBRATION, GROOMING, AND ALIGNIMENT PLAN}

\section{WITH CALIBRATION DATA}

\section{CONTENTS:}

1. Calibration, Grooming, and Alignment PTan for Project $\mathbf{W}-030$ (unreleased document).

2. Instrument Loop Signoff Sheets with list of applicable loop drawings (see note below).

3. Instrument loop calibration sheets, each followed by related field instrument cal. sheets.

4. Instrument cal. sheets for (non-loop) local instruments (if any).

\section{IMPORTANT NOTES:}

The attached data is presented as field-recorded by plant instrument technicians and initialed off by test engineers; it represents work done over many months according to current site work practices and best available information and techniques. Only necessary information was recorded for each calibration sheet, and thus many data fields are not applicable and are left blank. Some vendor-provided data is included and may be missing non-critical information. Test exception reports were created only where necessary.

Generally, "loops" refers to those instruments related to the central Monitor and Control System (MCS), although some non-MCS "local loops" are noted in the signoff sheets. Loop data sheets are attached in the order shown on the loop signoff sheets. Generally, each loop will include a loop-test data sheet, followed by its associated fieldinstrument calibration data sheet (with the most recent data. presented first) and any other relevant calibration information. Additional supporting data may be found in the archived original test records. 
CALIBRATION, GROOMING, AND ALIGNMENT PLAN

FOR PROJECT W-030

TANK FARM VENTILATION UPGRADE

PLAN APPROVAL

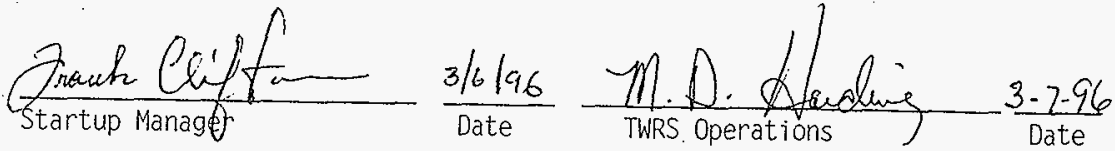

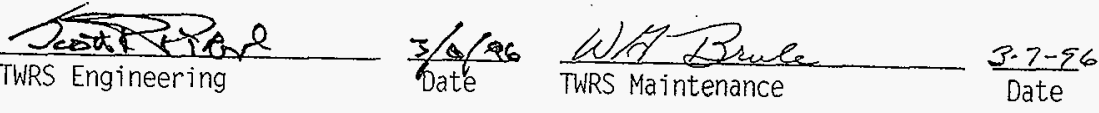

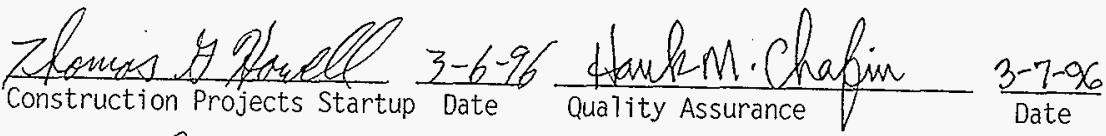

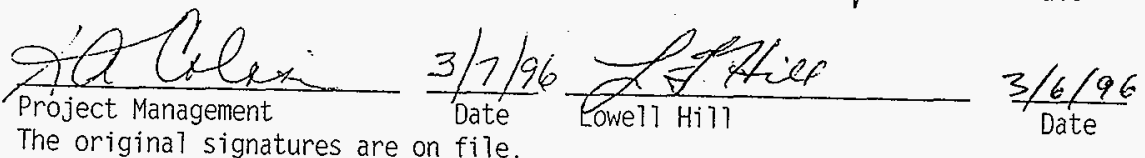




\section{TABLE OF CONTENTS}

SECTION NO.

PAGE

1.0 Purpose and Scope . . . . . . . . . . . . . . . . . 3

2.0 References ..................... 3

3.0 Personnel Requirements ................. 3

4.0 Change Control ................. 3

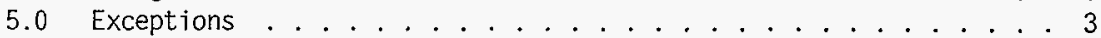

6.0 Precautions and Limitations . . . . . . . . . . . . . . . 4

7.0 Special Tools, Equipment, and Materials . . . . . . . . . . . 4

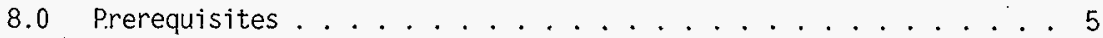

9.0 Procedure .................... 5

10.0 Restoration ................... . . . . . .

11.0 Records .................... . . 8

12.0 Attachments ................... 8

Attachment A- Raw Water System Test Package

Attachment B- Primary Ventilation Condensate System Test Package

Attachment C- Recirculation Condenser Cooling System Test Package

Attachment D- Recirculation Ventilation System Test Package

Attachment E- Primary Ventilation Condensate Cooling System Test Package

Attachment F- Primary Tank Ventilation Systern Test Package Attachment G- Vent Building Ventilation System Test Package 
The purpose of this Calibration, Grooming, and Alignment (CGA) Plan is to provide a safe, uniform, and expeditious method for verifying calibration of instrumentation and associated instrument loops in the Project W-030, Tank Farm Ventilation Upgrade.

1.1 The instrument loops will be calibrated on a system basis and will be identified in a list as part of each system CGA package.

\subsection{REFERENCES}

2.1 Drawing H-2-131000, Shts. 1 thru 4 (W-030 Drawing List).

2.2 Drawing ES-W030-Y00. Shts. 1 thru 5 (W030 MCS Logic Diagram Drawing List).

2.3 Memorandum of Understanding (MOU), Project $W$-030 Test Configuration Control. EDT No. 614741.

\subsection{CHANGE CONTROL}

Administrative or editorial changes required to this plan, during testing. may be accommodated as exceptions in the released test report. if the changes will not affect operating facility safety, function, or performance and will not compromise or influence test data. Addition or deletion of steps or major changes to this plan will be accomplished by revision and approval by the original signature authorities.

\subsection{EXCEPTIONS}

Exceptions to results or to the test procedure will be given a sequential number and recorded on Test Exception log sheet in the applicable CGA Pacakge attachment. A test exception report wi17 be filled out to record and disposition each test exception.

\subsection{PERSONNEL REQUIREMENTS}

5.1 Instrument Technician (2).

5.2 MCS Operator (1).

5.3. Electrician (as needed).

5.4 Test Engineer (1) 


\subsection{PRECAUTIONS AND LIMITATIONS}

6.1 During the performance of this procedure, if any of the following conditions are found. immediately stop work, place equipment in a safe condition, and notify the Test Engineer and take appropriate action to resolve the problem quickly:

6.1.1 Stop work if any condition could jeopardize personnel safety or jeopardize damage to equipment/components.

6.1.2 Stop work if personnel error or procedural error/inadequacy could prevent fulfillment of this procedure.

6.1.3 Stop work if any un-safe condition exists.

6.2 Review hazards in the work area (if any), prior to start of this test.

6.3 Sections or steps within sections of this procedure (except for prerequisites. Section 6.0) may be performed out of sequence under direction and authority of the Test Engineer.

6.4 Performance of this procedure requires stationing of an 0perator in the Control Room.

7.0 Special Tools, Equipment, and Materials

7.1 The following is a general list of test equipment that may be required to perform the testing under this procedure:

7.1.1 Digital Multimeter (OMM).

7.1.2 Decade Box.

7.1.3 Calibrated Pressure Source.

7.1.4 Calibrated Pressure Gauge.

7.1.5 Calibrated Power Supply.

7.1.6 Two-Way Radios or. Sound Powered Phones to communicate between field devices and Control Room. 
7.2 The Measuring and Test Equipment (M\&TE) used to collect test data during performance of this procedure shall meet the following requirements:

7.2.1 It shall be within its current calibration cycle as evidenced by an affixed calibration label.

7.2.2 It shall be capable of providing the desired range.

7.2.3 It shall have an accuracy consistent with state-of-the-art limitations. It shall be equal to or greater than the input tolerance specified on the Data Sheets or if device being calibrated is not recall related, at least 4 times greater than the specified device tolerance.

\subsection{PREREQUISITES}

8.1 Perform a pre-job meeting and walkdown prior to testing.

8.2 Identify safety concerns (if any) related to the testing and assure compliance with safety requirements.

8.3 Establish communications between field devices and Control Room.

8.4 Personnel radiological safety will be as specified by a Radiation Work Procedure for instrumentation/instrument loops requiring entry into the tank farm.

8.5 Equipment and materials entering a contamination control area shall be minimized.

\subsection{PROCEDURE}

\subsection{Test Boundaries}

This procedure provides boundaries within which the test personnel can work, allowing them flexibility to perform the testing and still work in a safe manner. These boundaries are as follows:

9.1.1 Test personnel must work within the site Lock and Tag procedures and identify those pieces of equipment that must be tagged out prior to the test.

9.1.2 Test personnel shall have the freedom to lift leads for diagnostic testing. The circuits being tested shall be restored to a normal configuration upon completion of testing or at the end of the shift, if testing of that loop does not continue on the next shift. 
9.1.3 Test personnel shall have the freedom to change the order in which the testing is being performed.

9.1.4 Test personnel shal1 have the authority to change the system configuration to match the drawing configuration if wiring is found not to be in accordance with the construction drawings and applicable ECN's on systems that have been turned over to WHC. The change must be noted/logged in the daily test log located in the control Room. The contractor should be notified in cases where the system has not been turned over to $\mathrm{WHC}$.

9.1.5 If the system configuration does not work as designed and is installed according to the contract drawings, the problem shall be noted in the daily test $\log$ and the design engineer shall be notified immediately to begin processing an ECN to correct the problem.

9.1.6 Test personnel, when completing a loop test, shall document it in the daily test $\log$ and initial for completion on the package instrument list. Also items found that are not installed per construction drawings, and any other problems/fixes shall be documented in the daily test log.

\subsection{Test Execution}

The test should be performed in accordance with the following steps:

9.2.1 The Test Engineer shal1 identify and initiate any system alignments that may be required for the loops to be tested.

9.2.2 Identify on Loop Test Data Sheet, the sensor actuation method to be used. Record the test instrument type (ie: pressure source, etc.). instrument number, model, and. calibration due date at the bottom of the Data Sheet (M\&TE Inst. No.).

9.2.3 Identify if instrument calibration has been performed or will be performed as part of the loop test. This information will be provided on the calibration data sheet attached to the Loop Test Data Sheet.

9.2.4 Connect the sensor actuation device to the loop to be tested. 
9.2.5 Loop tolerances are determined using the "Square Root of the Sum of the Squares (SRSS)" method as noted in ANSI/ASME PTC 19.1-1985. Part 1. "Measurement Uncertainties." and ANSI/ISA-S67.04-1988. Section 4.4.1. Loop tolerances will be pre-calculated and shown on the Loop Calibration Data Sheet.

9.2.6 Initiate the process input as shown on the Loop Calibration Data Sheet and then record the results in the spaces provided (LOI and MCS Screens) as applicable.

9.2.7 Verify annunciator setpoints and record the actual setpoint in the space provided on the Loop Test Data Sheet:

9.2.8 Additional remarks or comments may be recorded in the space provided on the Loop Test Data Sheet.

9.2.9 If there are any problems with the test that cannot be resolved quickly, then initiate a Test Exception on the form provided as part of each test package.

9.2.10 When al1 loop tests for a system have been completed, then the Test Engineer shall review the test data and sign in the space titled "Loop Complete" on Loop Test Data Sheets.

9.2.11 The associated P\&ID shall be highlighted as loops are completed as a method of tracking test completion.

9.3 DOCUMENT CONFIGURATION CONTROL

All personnel involved with this CGA Procedure and the actual testing shall handle all documentation in accordance with EDT No. 614741. Memorandum of Understanding, Project W-030 Test Configuration Control. The Test Engineer shall review this EDT with all personnel involved in the testing to ensure everyone understands the scope of this document.

\subsection{RESTORATION}

10.1 Upon completion of loop testing, restore the loop to it's design configuration.

10.2 Remove/disconnect all test equipment used to perform the loop test.

10.3 Verify loop restoration by observing that loop indications, alarms and/or computer points are consistent with expected conditions. 
HNF-SD-W030-TD-003,

11.0 RECORDS

REV. 0, PAGE 140

11.1 A11 data sheets, test exception logs and test exception forms will become part of the system preoperational test report that will be developed upon completion of each system Preoperational Test Procedure.

\subsection{ATTACHMENTS}

12.1 The attachments to this document will be laid out by system and will contain the following items for each instrument loop in that system:
- Instrument Loop Sign-Off Sheet
- Instrument Test Data Sheet
- Loop Calibration Data Sheet
- System P\&ID
- Test Exception Log
- Test Exception Form

12.2 The following system test packages will be developed for testing and will become attachments to this document:

Attachment AAttachment BAttachment CAttachment DAttachment E-

Attachment FAttachment G-
Raw Water System Test Package Primary Ventilation Condensate System Test Package Recirculation Condenser Cooling System Test Package Recirculation Ventilation System Test Package Primary Ventilation Condensate Cooling System Test Package

Primary Tank Ventilation System Test Package Vent Building Ventilation System Test Package 
HNF-SD-W030-TD-003,

REV. 0, PAGE 141

\section{INSTRUMENT LOOP SIGN-OFF SHEET}

for the

RECIRCULATION CONDENSER COOLING SYSTEM

\section{LOOP DIAGRAMS}

H.2-131333 Sht's. 3 thru 6

H-2.131333 Sht. 11

H-2-131333 Sht's. 16 thru 17

H.2-131334 Sht's. 3 thru 6

H-2-131334 Sht. 11

H.2.131334 Sht's. 16 thru 17

NOTES ON LOOP CAL DATA SHEETS:

A. Loop Cat Sheets -

1. Location field was not generally used and is left $b$ lank because the information was not helpful or needed.

2. Where "screen" fields are left blank, they are not applicable (i.e. Screen2, screen3). Applicable screen fields have a screen name given.

3. Where "Trip point" fields are left blank, they are not applicable.

B. Instrument Cal Sheets -

1. Sheets marked "VOID" have been superseded by more recent calibration and are included only to show that the instrument had been calibrated at time of PoTp test.

2. If the term "broken" is noted, then the instrument was later found defective and was replaced at time -... of iater calibration. 


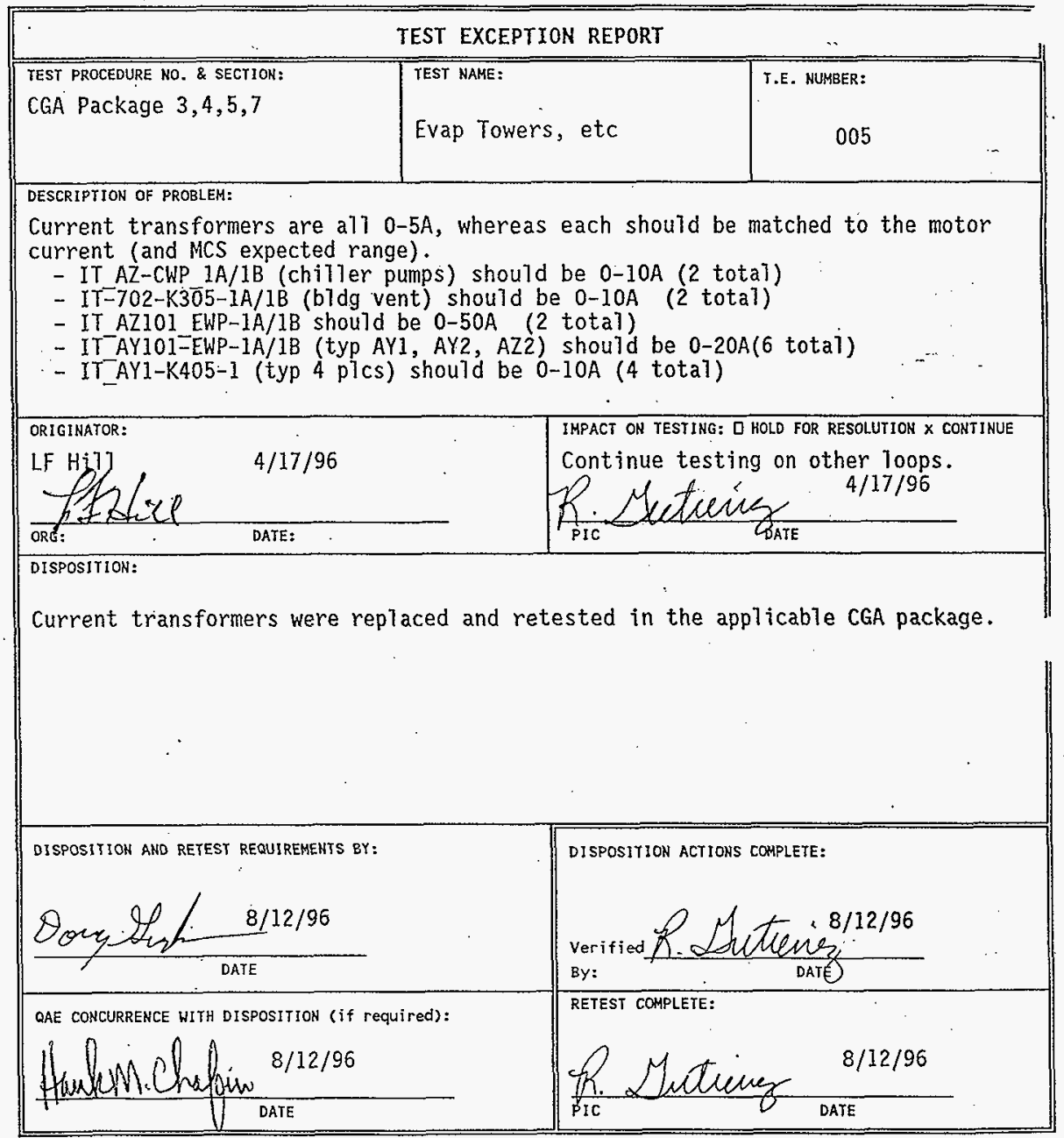




$$
\begin{aligned}
& \text { HNF-SD-W030-TD-003, } \\
& \text { REV. O, PAGE } 143
\end{aligned}
$$

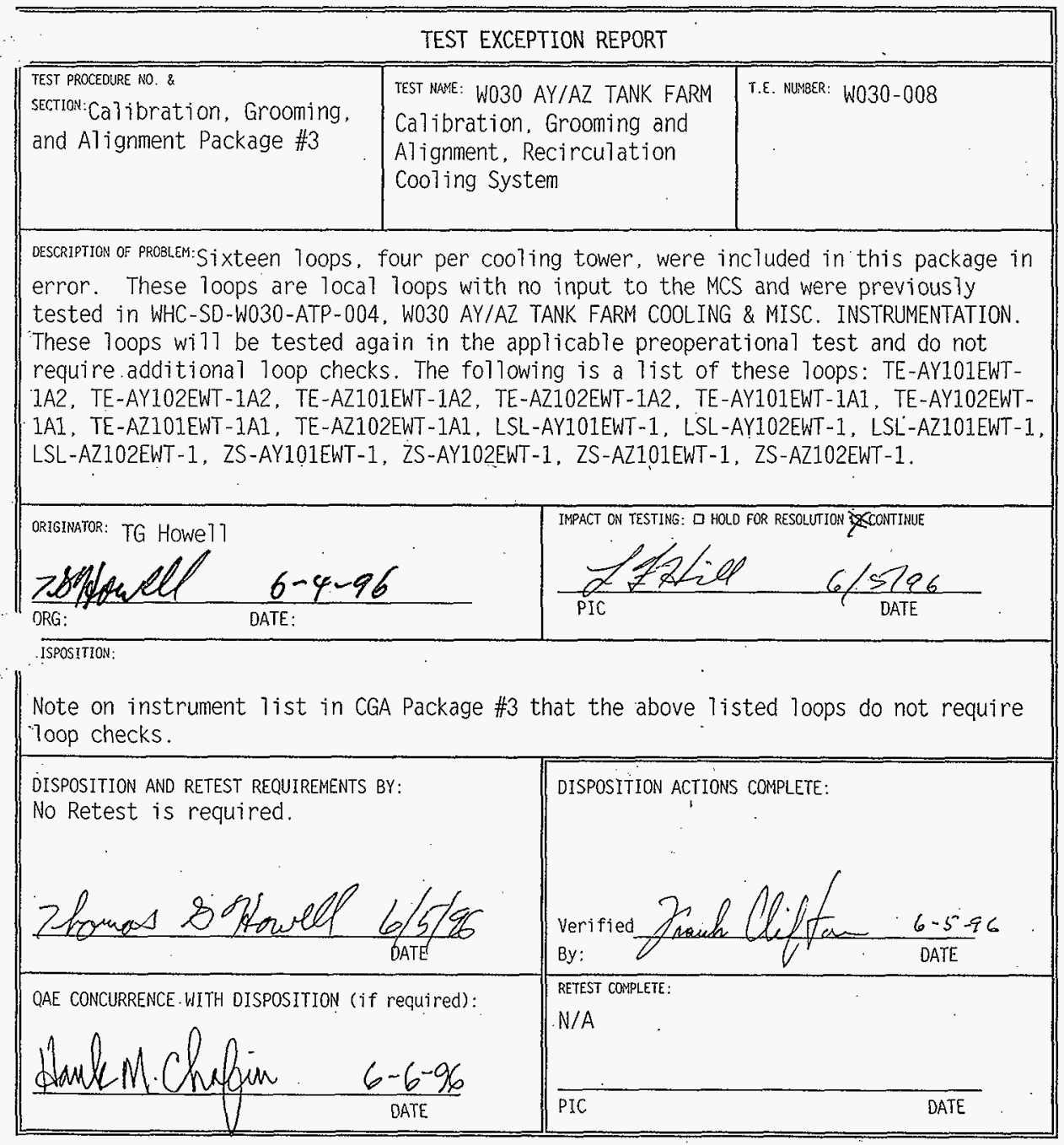


CGA PACKAGE \#3 INSTRUMENT LIST

HNF-SD-W030-TD-003,

REV. 0, PAGE 144

RECIRCULATION CONDENSER COOLING SYSTEM

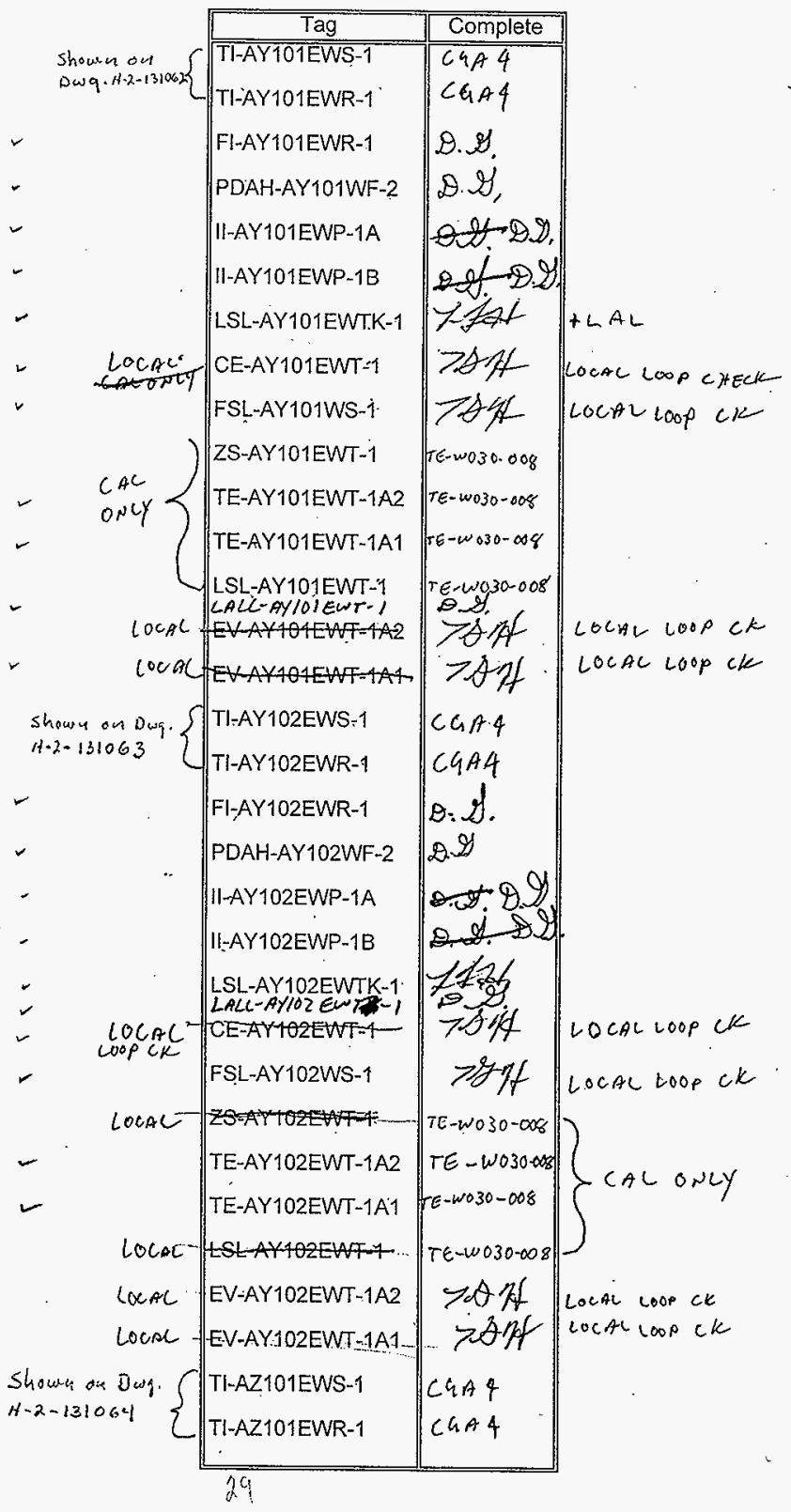

Page Number : 
CGA PACKAGE \#3 INSTRUMENT LIST

HNF-SD-W030-TD-003,

REV. O, PAGE 145

RECIRCULATION CONDENSER COOLING SYSTEM

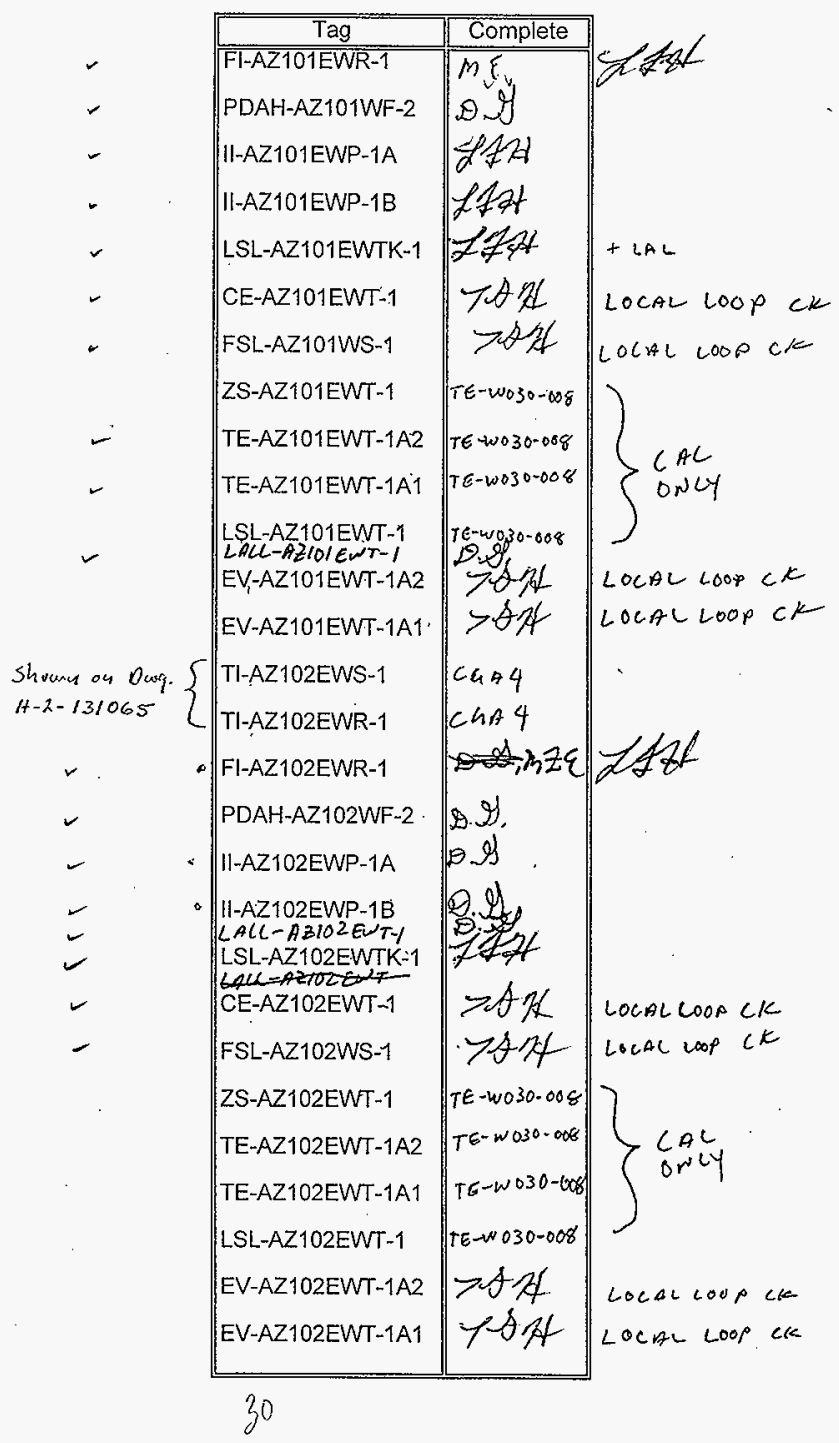

Page Number: 2 
CGA PACKAGE \#3INSTRUMENT LIST

RECIRCULATION CONDENSER COOLING SYSTEM

\begin{tabular}{|c|c|c|}
\hline & TAG & Complete \\
\hline - & YS_AY1_EWSP_1 & DSS \\
\hline- & YS_AY1_EWT_1 & $\theta$ I \\
\hline - & YS_AY1_EWP_1AI & D. I \\
\hline$\checkmark$ & YS_AY1_EWP_1B1 & D. थै, \\
\hline 一 & AY1_EWP_BAA & Q.1. \\
\hline- & AY1_EWP_IBB & Q. \\
\hline - & $A Y{ }_{1}$ EWSP_IA & $\theta 91$ \\
\hline- & $A Y 1$ EWSP_1B & 0.2 \\
\hline- & AY1_EWT_1A & of 8 \\
\hline 乞 & AY1_EWT_1B & $\theta$ \&. \\
\hline 2 & $Y S$ AY2 EWSP. 1 & D. \\
\hline - & YS_AY2.EWT_1 & D. \\
\hline 2 & $Y S \_A Y Z_{2} E W P$-1AI & $0 \%$ \\
\hline$\checkmark$ & YS_AYY_EWP_1B1 & b. \\
\hline 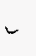 & AY2_EWT_1B & BS. \\
\hline r & AY2_EWT_IA & $p H$. \\
\hline$r$ & AY2_EWSP_1A & $\theta \mathscr{y}$ \\
\hline - & AY2_EWSP_1B & $\theta .4$ \\
\hline$\checkmark$ & AY2_EWP_1AA & DI. \\
\hline - & $A Y 2 \_E W P \_1 A B$ & 0. \\
\hline - & AY2_EWP_1BA & Dy \\
\hline$\sim$ & AY2_EWP_1BB & 9.28, \\
\hline$r$ & YS_AZ1_EWSP 1 & 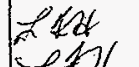 \\
\hline 一 & YS AZI EWP $1 \mathrm{~A} 1$ & $\mid \begin{array}{l}2 \\
x\end{array}$ \\
\hline
\end{tabular}


CGA PACKAGE \#3INSTRUMENT LIST

RECIRCULATION CONDENSER COOLING SYSTEM

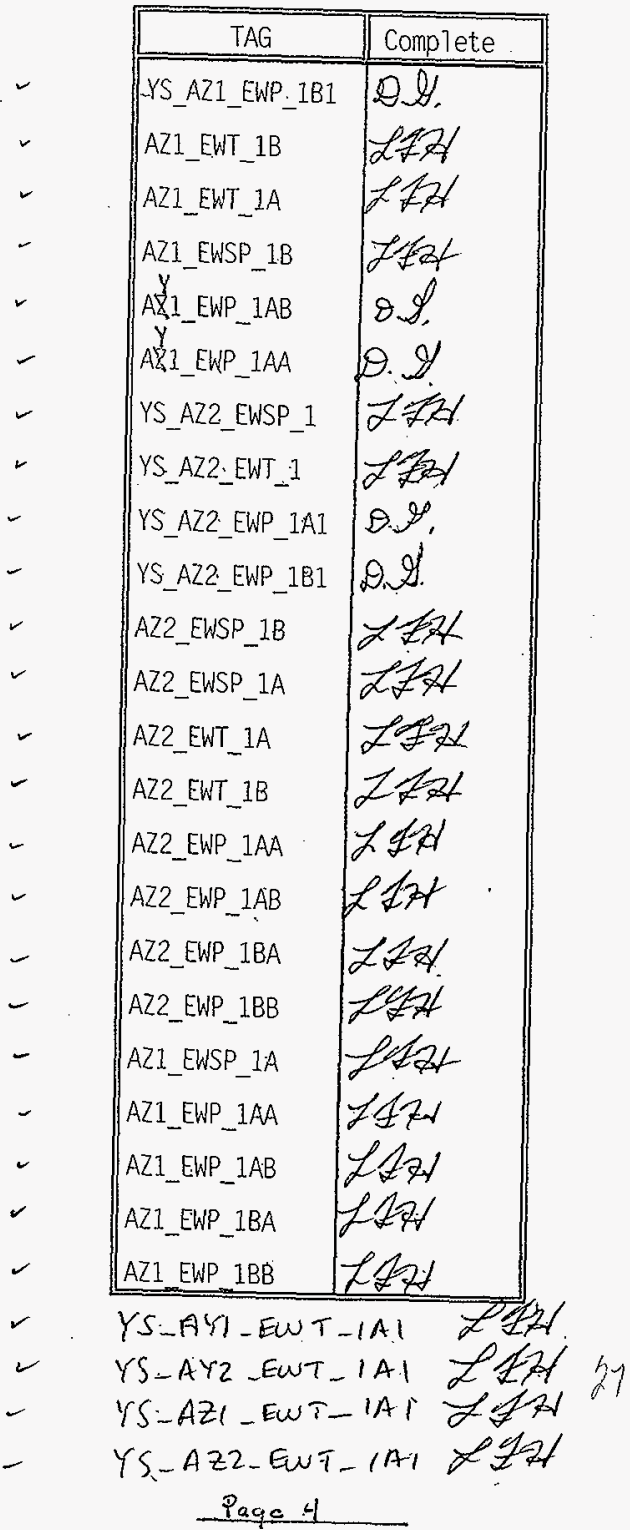


Tag: FI_AY1_EWR_1 I/0 Type: AI Description: EwrHdr Flow

Cntlr: 46 Chan No: 10

P\&ID: H-2-131067

Logic:

Remaxks :

Location:

Actuation Method: $4-20 \mathrm{~m}_{\mathrm{A}}$ Simularor

Sensor Galibration:

Q 5 pt cal

$\square$ per procedure

VI DATA (data attached)

$\frac{Q .2}{(\text { sig }}$

Loop Tolexance:

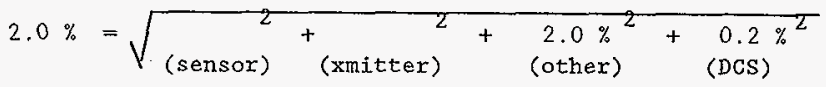

Units: GPM

Process Input

Lo: $\quad 0.00$

Hi: $\quad 300.00$

$$
\begin{array}{ccc}
\text { Readout Tolerance } & \begin{array}{c}
\text { LOI } \\
\text { LCU4-16 }
\end{array} & \begin{array}{c}
\text { Screen1 } \\
\text { O6EvpAY1 }
\end{array} \\
-6.03-6.03 & \cdots .32=0.38 & -.0 .30 \\
293.97-306.03 & \underline{298.88} & -299.72
\end{array}
$$

\begin{tabular}{|c|c|c|c|c|}
\hline Annunciator $(s)$ : & Priority & Setpoint & Tolerance & Trip Point \\
\hline Max: & 0 & 99999.99 & - & \\
\hline HiHi: & 0 & 99999.99 & - & \\
\hline $\mathrm{Hi}:$ & 0 & 99999.99 & - & \\
\hline Lo: & 2 & 170.00 & $163.97-176.03$ & $-180.0 .169 .96(13.09 \mathrm{ma}$ \\
\hline LoLo: & 0 & -9999.99 & - & \\
\hline Min: & 0 & -9999.99 & - & \\
\hline
\end{tabular}

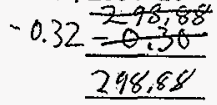

Screen1 Screen2 Screen3

Other: TESTEDTOTALIZER ANO IT PASSED

IKGAL

MTE Instr. No: $817-23-01-005$ Due Date: $1 / 2 / 92$

(signoff) Loop Complete: D. L) Date: $4 / 12 / 96$ 
$5 / 1 / 97$

W-030 CALIBRATION DATA SHEET

HNF-SD-W030-TD-003,

REV. 0, PAGE 149

Tag: FT-AY101EWR-1

P\&ID: H-2-131067. SH 1

LOOP: $\quad H-2-131333, \mathrm{SH} 11$

Manufacturer: Fisher Potter Model No.: 501W.3212B1JB_ Serial No: 93 w0170

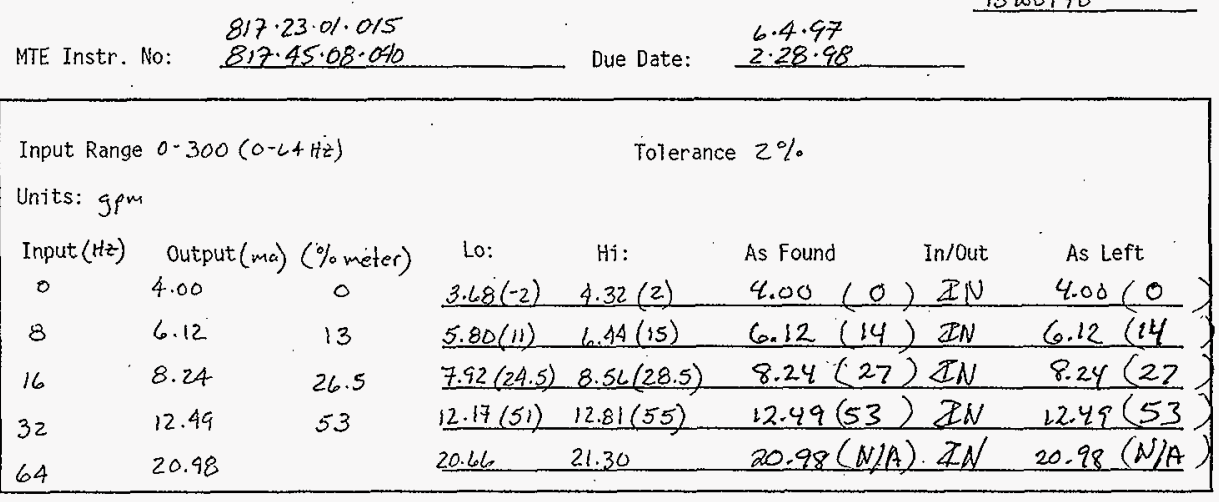

(signoff) calibrated By: Fitzsinmons/Zylonske $\quad$ Date: $5 \cdot 16-97$

Location: LIr AV Coding Water Tower

$$
\begin{aligned}
\text { Comments } & \text { Calibration factor }(\bar{K})=24.115 \mathrm{C} / \mathrm{gal} \odot 93^{\circ} \mathrm{F} \\
& f_{0}=120.6 \mathrm{~Hz} \\
& \text { Span factor }=1324 \\
& \text { fest }=60.3 \mathrm{~Hz}(\max )
\end{aligned}
$$

Verified $O$ and Span readings at MICON.

$$
\begin{gathered}
0=0 \text { g pm } \\
53 \%(159 \mathrm{gpm})=159 \mathrm{gpm}
\end{gathered}
$$


Tag: PDAH_AYIWE_2 I/O TYpe: DI

Description: AYlEWFtrDpHi.

Cntir:

Remarks :

Location:

Actuation method: Pressure, sounce, Regulated Prossure suurce. Instrument listed below.

Sensor Calibration:

区

Setpoint:

Loop Tolerance:

\begin{tabular}{|c|c|c|c|c|c|}
\hline & & & & IOI & Screen 1 \\
\hline & Process & State & color & LCU4-15 & 06EVPAY 1 \\
\hline Lo & (0): & NORMAI & 2 & GFF & GREEN \\
\hline Hi & (1): & HIGH & 3 & $O N$ & low \\
\hline
\end{tabular}

Annunciator (s): Priority

$\begin{array}{rcc}\text { Annunciator (s) : } & \text { Priority } & \text { Alarm color (actual) } \\ \text { Hi: } & 2 & \text { fellow } \\ \text { IO: } & 0 & \end{array}$

(ata attached) $\frac{\mathcal{S} \text { (signoff) }}{\text { (sign }}$

$15 \mathrm{psi}$

Other:

ME Instr. No: $817-35-40-031$ Due Date: $6-22-96$ (signoff) Loop complete: 4.91. Date: $4 / 16 / 96$ 
Manufacturer:

Model No.:

Serial No:

MTE Instr. No: $817-35-40-031$

Due Date:

Input Range $0-20$

Tolerance $5 \%$

Units: psi

Input output

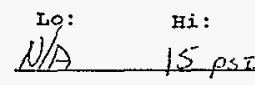

Hi:
15 psi.

As Found

In/out

As Ieft

New. IN 15 pst

(signofficazibrated вy: Kevi- Dis Pat Husen

Date: $4-16-96$

Location:

Comments

SET SWITCH TO ALARM AT 15PSID

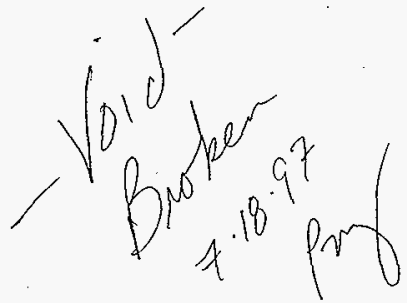


Tag: PDIS-AY101WF-2

P\&ID : P-W030-P1

Model No. : $43220 \mathrm{C}$
LOOP: H-2-131333, SH 17

Serial No: ZE-000 767 MTE Instr. No: $817-3-40-03 /$ Due Date: $-7-3 /-87$

Input Range $0-20$

Units: PSI

Input output

$\begin{array}{ll}0 & 0.00\end{array}$

$5 \quad 5.00$

$10 \quad 10.00$

$15 \quad 15.00$

$20 \quad 20.00$
Tolerance $2.00 \%$

$$
+/-0.40
$$

Lo: Hi: As Found In/Out As Left

$\begin{array}{llllll}-0.40 & 0.40 & 0.0 & I k & 0.0\end{array}$

\begin{tabular}{|c|c|c|c|c|}
\hline 4.60 & 5.40 & 49 & $I_{\mu}$ & 5.1 \\
\hline 9.60 & 10.40 & 9.9 & In & 10.1 \\
\hline 14.60 & 15.40 & 15,2 & In & 2501 \\
\hline 19.60 & 20.40 & 20,2 & Zn & $20 \times$ \\
\hline
\end{tabular}

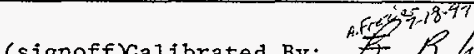

(signoff)Galibrated By:

Location: AYI01 GOAPISTR $E$ TOWER
Comments

SET SWITCH TO ALARM AT,15PSI, FUNCTIONAL TEST: DOES ALARM COME IN AT MICON? YES ,NO

Power not avallable $\Rightarrow$ Limited cal. 
$4 / 17 / 96$

W-030 LOOP TEST DATA SHEET

HNF-SD-W030-TD-003,

REV. 0, PAGE 153

Tag: II_AY1_EWP_1A I/O TYpe: AI Description: EwPumplACurrent

Cntlx: 46 Chan No: 11 P\&ID: H-2-131067 Logic: Y400

Remarks :

Location:

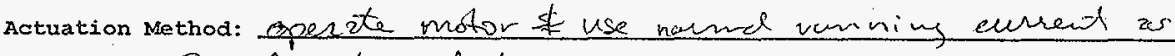
sean $p$ t. to clad

sensor calibration: $N / A$ - factory cellareted only

$\square 5$ pt cal.

per procedure

(data attached)

(signoff)

Loop Tolerance:

Units: AMP

Process Input

Readout Tolerance

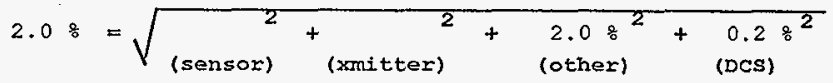

Ir: 0.00

$-.40-.40$

Hi: -20.00

Running current

9.6

$9 \cdot 2-10.0$

Annunciator (s):

Priority setpoint

Tolerance

Trip Point

Max: $\quad 0 \quad 99999.99$

Hill: $0 \quad 99999.99$

MOI
ICU 4-16
$\frac{0}{\frac{9}{x}}$

screen

Screen

$t \infty 3$

Ex: $\quad 0 \quad 99999.99$

I io: $\quad 0 \quad-9999.99$

LIlo: $\quad 0 \quad-9999.99$

Min: $\quad 0 \quad-9999.99$

other:

* AVE vide since current secillater $\triangle \pm .2 A$

MTE Instr. No: $988-45-02-006$ Due Date: $1-24-97$

(signoff) Loop Complete:

Date: $5 / 14 / 96$ 
Tag: IT-AY101EWP-IA

Manufacturex: KATY INSTRS Model No.:420 Serial No: $617 \cdot 45 \cdot 02 \cdot 006$ MTE Instr. No: $817 \cdot 45 \cdot 08 \cdot 040$ Due Date: $\begin{aligned} & 5 \cdot 16 \cdot 97 \\ & 2.28 \cdot 98\end{aligned}$

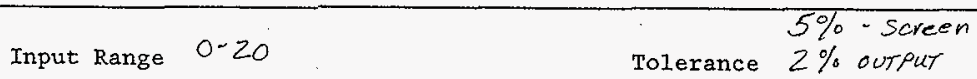

20 $20.00 \quad 20$

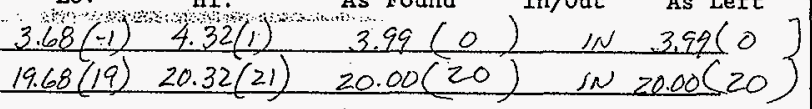

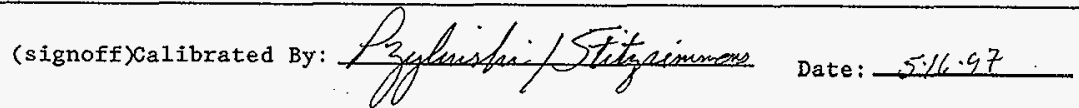
Location: IOL AY COOLNG WATER TOWER (PUMP IA)

comments FACTORY CAUBRATED; check, zero: SPAN. Use current generator $\omega$ calibrated MTE clamp on meter 
$\because \quad 4 / 17 / 96$

W-030 LOOP TEST DATA SHEET

$$
\begin{aligned}
& \text { HNF-SD-W030-TD-003, } \\
& \text { REV. 0, PAGE } 155
\end{aligned}
$$

Tag: II_AY1_EWP_1B IB IO Type: AI

Description: EwPump1BCurrent

Cutler: 46 Chan No: 12

P\&ID: $H-2-131067$

Logic: $\quad Y 402$

Remarks :

Location:

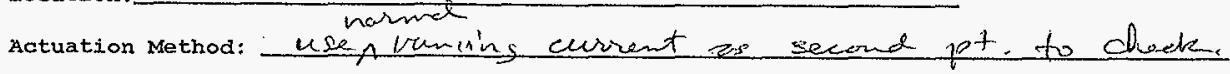

sensor calibration: N/A Not calibiatoble (Fetor col ar ty) [D $5 \cdot \mathrm{pt}$ cal

per procedure (data attached) (signoff)

Loop Tolerance:

Units: AMP

Process Input Readout Tolerance

Lo: $0.00-.40-.40$

Hi: 20.00

$19.60-20.40$

Running 9.3

$8.9-9.7$

current

Annunciator (s) :

priority setpoint

Max: 009999.99

HiED : $\quad 0 \quad 99999.99$

Hi: $\quad 0 \quad 99999.99$

Lo: $\quad 0 \quad-9999.99$

JOLO: 0 -9999.99

Min: $\quad 0 \quad-9999.99$

Tolerance Trip Point

Tolerance Trip Point

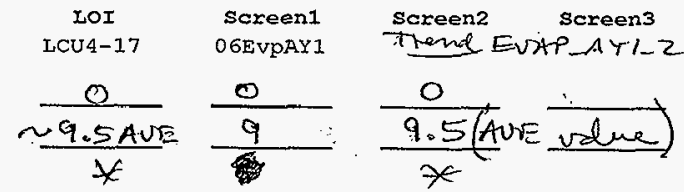

(DOS)

other: turnip current oscillates $\sim \pm .2 \mathrm{~A}$

Tothizer overview swears pump fill when for openerMIE Instr. No: $988-45-02-006$ Due Date: $1-24-97$

(signoff) Loop Complete:

Date:

$5 / 1496$ 
Manufacturer: KATY INSTRS. Model No.:

420 Serfal No:

\section{$877 \cdot 45 \cdot 02 \cdot 004$}

MTE Instr. No: $817 \cdot 45 \cdot 08 \cdot 040$

Due Date: $\begin{array}{r}2 \cdot 28 \cdot 98 \\ \hline\end{array}$

\section{$5 \%-$ screen}

Input Range $0-20$

Tolexance $2 \%$-OUTPUT

Units: Amps

Input (A) output (ma)(micon) Lo: Hi: As Found In/Out As Left

$\begin{array}{llll}0 & 4.00 \quad 0 \quad 3.68(-1) \quad 4.32(1)\end{array}$ $3.99(0)$ in $3.99(0)$ $20 \quad 20.00 \quad 20 \quad 19.68(19) 20.32(21) \quad 19.98(20) \quad \mathrm{N} 19.98(20)$

(signoff)Galibrated By:

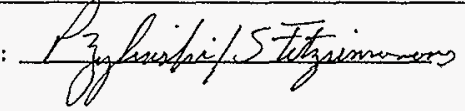

Date: $5 \cdot 16 \cdot 97$

Location: 101AY Coolint WATER Towier (Pump 1B)

comments Factory calibrated, check zero; SPAN. Use current generator w/calibrated MTE clamp on meter 
$3 / 28 / 96$

W-03O LOOP TEST DATA SHEET

HNF-SD-W030-TD-003,

REV. 0, PAGE 157

Tag: LAL_AY1_EWTK_I I/O TYpe: DI Description: AYIEWTWrLVILO

CutIs: 47 Chan No: 23 P\&ID: H-2-131067 LOgic:

Remarks :

Location:

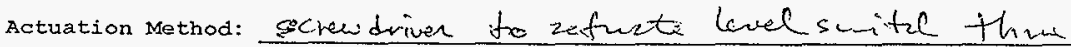
test port provided.

Sensor Calibration:

per procedure Mlotcilibutzble (data attached)

(signoff)

Setpoint:

Loop Tolerance:

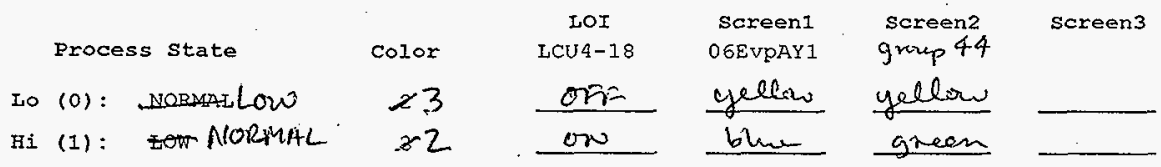

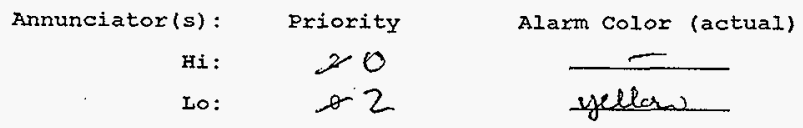

revised per kist

other:

MTE Instr. No: Due Date:

(signoff) LoOp complete:

Date:

$12 / 96$ 
Tag: LSL-AY101EWTK-1

P\&ID: H-2-131067, SH 1

LOOP: $\quad H-2-131333, \mathrm{SH} 16$

Manufacturer: IIT MCDONAlELL Model No.: IMILLER

MTE Instr, No:

L/A Due Date: _ L N/A

Input Range Functional test

Units: Sw/TCH.

Tolerance $N / A$

Input loutput Lo: Hi: As Found in/out As Left

Mect.

TRIP SWITCH

"SEE NSTRUCTONS IN COMMEATS"

(signoff) Calibrated By:

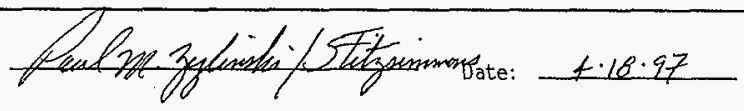

Location: $\quad$ IOIAY COOLWG LLATER TOWER.

Comments

Instructions: Actuate level switch with screwdriver through access port provided. Verify LAL (YELLOW) Recieved on Micon Screen.

Is AlARM Received at MiCON? YYN 
G: CE/CSH-AY101EWT-1 I/0 Type: N/A

Description: Tower Cond.

P\&ID: $\mathrm{H}-2-131067$

Remarks: Local loop on AY101 Cooling Tower with no input to the MCS. Controls conductivity of the tower water via feed and bleed.

Location: Conductivity element in cooling tower filtration 10op. Conductivity switch/controller located in panel next to local tower control panel

Actuation Method: Simulate conductivity changes with a decade box and verify that the controller will open the tower drain valve on high conductivity and close the drain valve when conductivity is below setpoint (1000 umho)

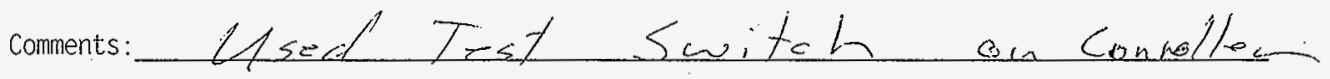

MTE Instr. No:

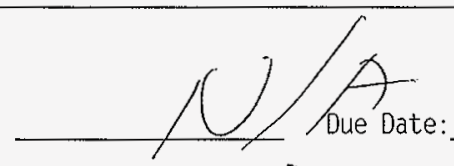

(signoff) Loop Complete:

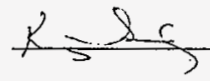

Date: 
Tag: CSH-AY101EWT-1

P\&ID: $H-2-131067$. SH 1

LOOP: W-030-P1

Manufacturer: IDEX Model No.: SHICE FACX Serial No: SE-7X4 MTE Instr. No: Buffer soln- 7070 Due Date: lamites cal.

Input Range $0-6000 \quad$ Tolerance $2 \%$

Units: Mmho's/cm

7.1 .2 Input Output $\mathrm{LO}$ : $\mathrm{Hi}: \quad$ As Found In/Out As Left

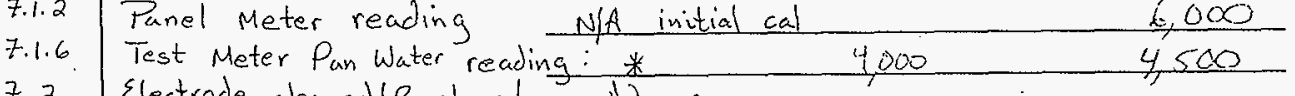

7.2 Electrode cleaned/Replaced New?

7.3

7.3.2 As faund trip point Panel Meter reading

7.4.2. Control indicator Light Panel Meter reading 2432 wminil

7.4- Retay-replaced- $(-y) N A)=$ ing 6.11 .96

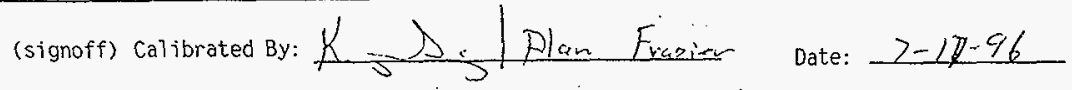

Location: AY. Cooling water Towers

7.1 .8 Conductivity controller panel reading $\frac{4.500}{11}$.

7.1 .9 Is cond meter $(*)$ and conductivity controller within $2 \%(y)$

* Buffer sohn. used while system S/D and dramed.

7.4.5 Is the reading from 7.4.2-withing of of specified trip point setting? $(y / N) . N)$ 
P\&IO:H-2-131067

Remarks: Loca l loop with no inputs to the MCS. Interupts conductivity controls if there is no flow established through the conductivity cell.

Location: Flow switch located at AY101 cooling tower in filtration loop downstream of conductivity element.

Actuation Method:

Actuate switch locally or simulate switch actuation using electrical jumper. verify that a no flow condition prevents the conductivity cell from acutating the tower drain valve on high conductivity.

Comments:

$$
\text { Used Flow through System }
$$

MTE Instr. No:

Due Date:

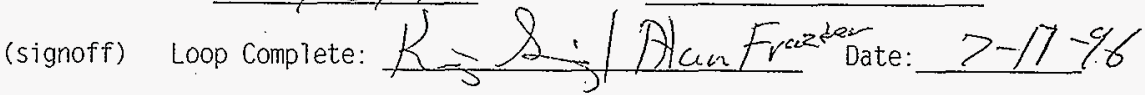


Tag: FSL-AY101WS-1.

P\&ID: H-2-131067, SH 1

LOOP:

Manufacturer: IDEx/Pitsafeeder Model No.: Option 3 Serial No: SFZZZ MTE Instr. No: Due Date:

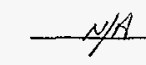

Units:

Input

output

Lo:

Hi:

As Found

In/out

As Left

Finctional

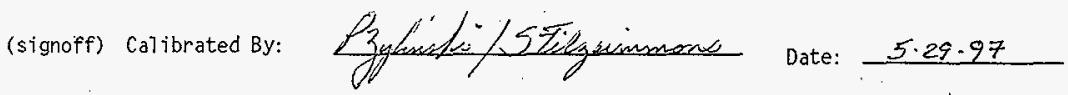

Location: 101 AY Cooling Water Tewer

comments * De-energize circ fump to cause low flow.

Does flow light on Conductivitymeter (CSH-AYIOIEWT-I) light up when Flow surtch is activated? $\mathrm{N} N$

* Manually activated switch while system is SlD and drained 
Manufacturer: Juhnson Controls Mode1 No.: ABфABA-44 serial No: 25-000-701 $817-13.55 .025$

MTE Instr. No: $\frac{817 \cdot 13 \cdot 55-014}{817 \cdot 45 \cdot 08 \cdot 0 / 4}$ 817.45 .08 .044

Due Date: $\frac{16-18-97}{2-12-98}$

Py s.m.s

Input Range 60-140

Tolerance NHA Z\%

Units: DEG F

Input Output

Lo:

$\mathrm{Hi}:$

As Found

In/Out

As Left

$100^{\circ} \mathrm{F}$ Switch

98.4 106.6 100.6 100.6

(signoff)Calibrated By: Fitzsimmons/zylinske

Date: $4-45-q 7$

Location: LOLAY Cooling Water Tower

Comments Damper Control, set to 'B' span for testing - begins opening CALIBRATE PER VENDOR DATA SET TO TURN ON AT 100 DEG F 
$3 / 8 / 96$

LIMITED

W-030 CALIBRATION DATA SHEET

HNF-SD-W030-TD-003,

REV. 0, PAGE 164

Tag: FE-AY101EWT-1A1

P\&ID: H-2-131067, SH 1

LOOP:

$2 E \cdot 000-697$

Manufacturer: Johnson Controls model No.: A/9ANC-25 serial No:

$$
817-45-08-040
$$

MTE Instr. No: $778-13-55-002$

2.5.97

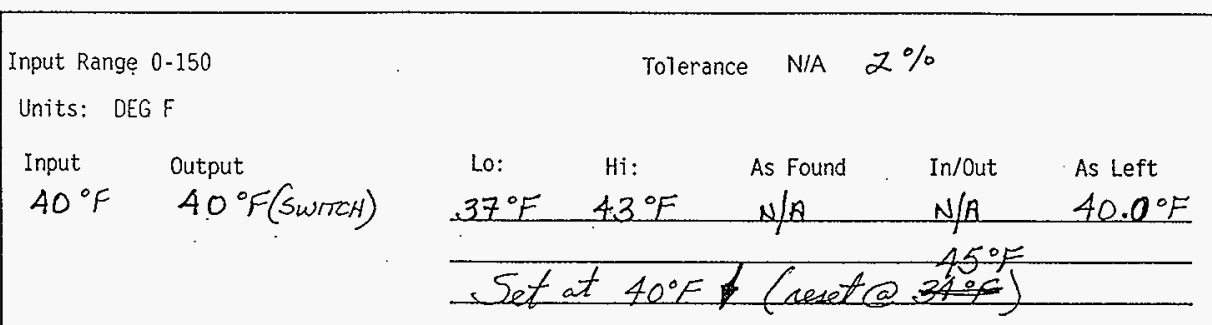

(signoff) Calibrated By:

Date: $3-14-96$

Location: 101AY Coking Toured

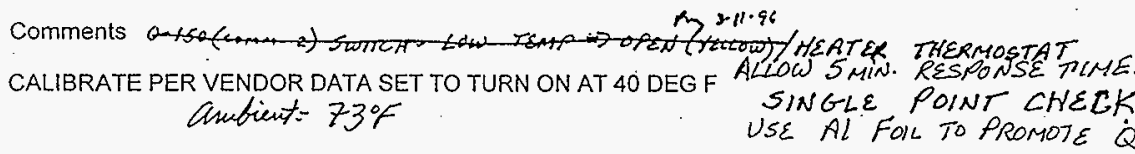

Note 1: Wired ned (common) to yellow (close on temp a A), Wiring (electrical) diagram shows yellow -in hire". w/ heater lome

- Please chick to make sure this isis opposite desired position.!!!

Notes: Response tine required due to long length of copsillayy tube (min. $=5$ min) 
Tag: TS-AYIO1EWT-1AI

P\&ID : H-2-131067, SH I

LOOP:

Manufacturer: Juhnson Controls Mode1 No.: Al9ANC-25 Serial No: $2 E-000 \cdot 697$ $817-13.55 .025$

MTE Instr. No: $817-13-55-014$ $7 \cdot 24-97$

\section{$817 \cdot 45 \cdot 08-044$}

Due Date: $11 \times 18.97$ $2 \cdot 12-98$

Input Range 0-150

Tolerance N/A

Units : DEG F

Input

$40^{\circ} \mathrm{F} \quad 40^{\circ} \mathrm{F}$ (Switd)

\begin{tabular}{llrrr} 
Lo: & Hi: & As Found & In/Out & As left \\
$37^{\circ} \mathrm{F}$ & $43^{\circ} \mathrm{F}$ & 43.0 & IN & 40.1 \\
\hline
\end{tabular}

Set at $40^{\circ} \nabla\left(\operatorname{resetat} 45^{\circ} \mathrm{F}\right)$

(signoff)Calibrated By: Fitesimmans

Date: $3-19-97$

Location: 101AY Cooling Tower

Comments

CALIBRATE PER VENDOR DATA SET TO TURN ON AT 40 DEG F 
Tag: IAALI_AY1EWT_1

Logic:

Remarks :

Location:

Actuation Method: TURNEP LEAK DETECTON QN ANO OFF

sensor calibration: $N / A$

$\square$ per procedure (data attached)

Setpoint:

Loop Tolerance:

\begin{tabular}{|c|c|c|c|c|c|c|}
\hline & Process state & Color & $\begin{array}{c}\text { LOI } \\
\text { LCU4-15 }\end{array}$ & $\begin{array}{c}\text { Screen } 1 \\
\text { 06EvpAY1 }\end{array}$ & Screen2 & screen 3 \\
\hline Lo & (0): & 3 & OFF & Low & & \\
\hline Ei & NORMAL & 2 & $O N$ & $N O R M A C$ & & \\
\hline
\end{tabular}

\begin{tabular}{|c|c|c|}
\hline Annunciator (s): & Priority & Alarm Color (actual) \\
\hline Hi: & 0 & - \\
\hline Lo: & 2 & $y \leq C \mathrm{COW}$ \\
\hline
\end{tabular}

other:

MPE Instr. No: 
Tag: FI_AY2_EWR_1 I/O Type: AI Description: EwrHdx Flow

Cntlx: 46 Chan No: 24 P\&ID: H-2-131068 Logic:

Remaxks :

Location:

Actuation Method: $4-20 \mathrm{~mA}$ simucoroc

Sensor Galibration:

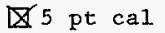

$\square$

per procedure $V I D g T A$ (data attached)

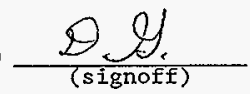

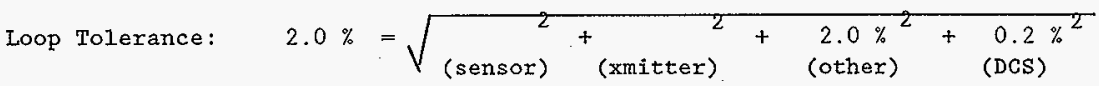

Units: GPM

Process Input

Lo: $\quad 0.00$

Hi: $\quad 300.00$
Readout Tolerance

$$
\begin{gathered}
-6.03-6.03 \\
293.97-306.03
\end{gathered}
$$

\begin{tabular}{|c|c|}
\hline LOI & Screen1 \\
\hline LCU4-22 & 07EvpAY2 \\
\hline$-0,35$ & $-0,02$ \\
\hline 298,92 & 299.40 \\
\hline
\end{tabular}

$\begin{array}{rcr}\text { Annunciator(s): } & \text { Priority } & \text { Setpoint } \\ \text { Max: } & 0 & 99999.99 \\ \text { HiHi: } & 0 & 99999.99 \\ \text { Hi: } & 0 & 99999.99 \\ \text { Lo: } & 2 & 170.00 \\ \text { LoLo: } & 0 & -9999.99 \\ \text { Min: } & 0 & -9999.99\end{array}$

Tolerance

Trip Point

$163.97-176.03$

169.83

Other:

Tested totalizer and It passea

$1 K G A C$

MTE Instr. No: 817-23-01-005 Due Date: $1 / 2 / 97$

(signoff) Loop Gomplete: $\quad \theta . \mathscr{g}$.

Date: $4 / 12 / 96$ 
$6 / 13 / 96$

W-030 CALIBRATION DATA SHE']

HNF-SD-W030-TD-003,

REV. 0, PAGE 168

Tag: FT-AY102EWR-1

P\&ID : $\mathrm{H}-2-131068$, SH 1

LOOP: H-2-131333, SH 11

Manufacturer: Fisher Porter Model No.: $504 \mathrm{~V}^{+3212815 B}$ serial No: $93 \mathrm{~W} 817043$

Moe Instr, No: $817 \cdot 45 \cdot 08 \cdot 040$

Due Date: $\begin{aligned} & 2-5-97 \\ & 6-4 \cdot 97\end{aligned}$

Input Range $0 \sim 300$

Tolerance $2 \%$

Units: gpu

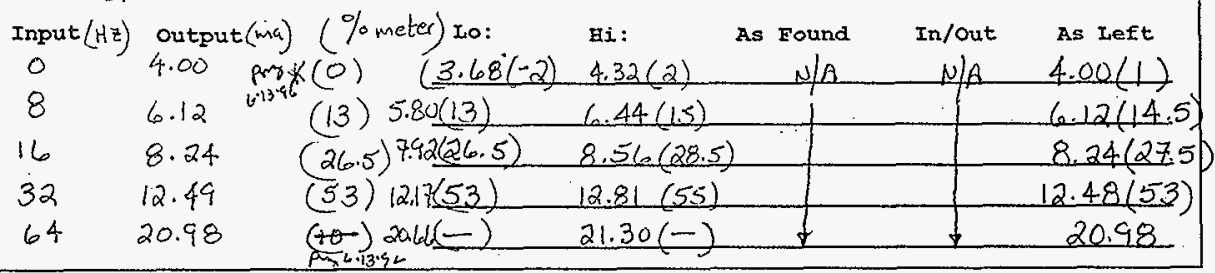

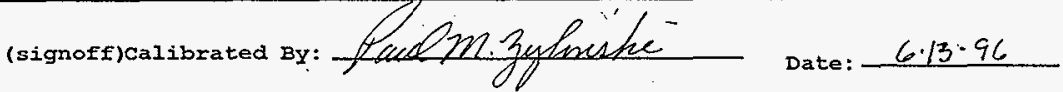

Location: 102AY COOWWG WBTER tower

$$
\begin{aligned}
& \text { comments Calibration factor }(\bar{K})=24.115 \mathrm{C} / \mathrm{gal} @ 93^{\circ} \mathrm{F} \\
& f_{0}=120.6 \mathrm{~Hz} \\
& \text { Span factor }=1324 \\
& f \text { test }=60.3 \mathrm{~Hz} \text { (max }
\end{aligned}
$$

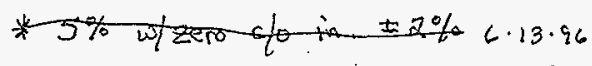

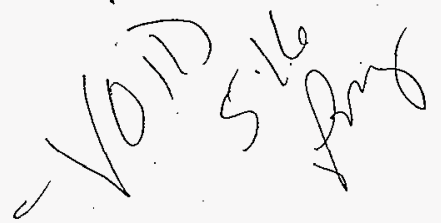


Tag: FT-AY102EWR-1

P\&ID: H-2-131068, SH 1

LOOP: $\mathrm{H}-2-131333, \mathrm{SH} 11$

Manufacturer: Fisher Porter Model No.: $50 \angle \mathrm{V}-32 / 2 \mathrm{~B} 1 \mathrm{JB}$ serial No:

MTE Instr. No: $817 \cdot 45 \cdot 08 \cdot 040$

\section{Due Date: $\quad \begin{array}{r}2.4 \cdot 97 \\ 2.28 \cdot 98\end{array}$}

$93 \omega \phi 17 \phi 43$

\section{che}

Input Range $0-300(0.64 \mathrm{~Hz})$

Tolerance $2 \%$

Units: gpm

\begin{tabular}{|c|c|c|c|c|c|c|c|c|}
\hline Input $(\mathrm{Hz})$ & \multicolumn{2}{|c|}{ Output (ma) ( $\%$ meter) } & Lo: & $\mathrm{Hi}$ : & As Found & & In/Out & As Left \\
\hline 0 & 4.00 & 0 & $3.68(-2)$ & $4.32(2)$ & 4.00 & $(0.5)$ & IN & $4.00(0.5)$ \\
\hline 8 & 6.12 & 13 & $5.80(18)$ & $6.44(15)$ & 6.11 & $(14.5)$ & $\alpha$ & $6.11(14.5)$ \\
\hline 16 & 8.24 & .26 .5 & $7.92(24.5)$ & $8.56(28.5)$ & 8.23 & $(28)$ & $I N$ & $8.23(28)$ \\
\hline 32 & 12.49 & 53 & $12.17(51)$ & $12.81(55)$ & 12.47 & $(53.5)$ & $I N$ & $12.47(53.5)$ \\
\hline 64 & 20.98 & & 20.62 & 21.30 & 20.96 & $(N / N)$ & IN & $20.96(\mathrm{~N} / \mathrm{A})$ \\
\hline
\end{tabular}

(signoff) Calibrated By:

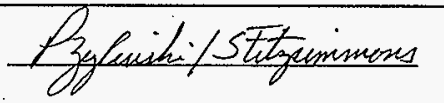

Date: $5 \cdot 16 \cdot 97$

Location: 102AY Cooling Water Tower

comments Calibration factor $(\bar{K})=24.115 \mathrm{c} / \mathrm{gal} @ 93^{\circ} \mathrm{F}$

$$
\begin{aligned}
& f_{0}=120.6 \mathrm{~Hz} \\
& S_{\text {pan factor }}=1324 \\
& f_{\text {test }}=60.3 \mathrm{~Hz} \text { (max) }
\end{aligned}
$$

Venfied $O$ and Span at MicoN. Pry

$$
\begin{gathered}
0=0 \mathrm{gpm} \\
53 \%(159 \mathrm{gpm})=158 \mathrm{gpm}
\end{gathered}
$$


Tag: PDAH_AY2WF_2 I/O TYPe: DI

Description: AY2EWFtrDpHi

Cntlr:

48 Chan No:

20

P\&ID: $H-2-131068$

Logic:

Remarks :

Location:

Actuation method: PrEESUre sounce, REGUCATED PRESSUR Source, Instrument isted below.

Sensor Calibration:

区

per procedure

(data attached)

(signoff)

point: $15 \mathrm{psi}$

Loop Tolerance:

\begin{tabular}{|c|c|c|c|c|c|c|c|}
\hline & & & & LOI & Screen 1 & Screen2 & Screen3 \\
\hline & Process & s state & Color & LCU 4-21 & 07ЕvрАY2 & & \\
\hline Lo & $(0):$ & NORMAL & 2 & OFF & G nEEN & & \\
\hline $\mathrm{Hi}$ & (1): & $\mathrm{HIGH}$ & 3 & $O N$ & Yelcow & & \\
\hline
\end{tabular}

$\begin{array}{rcc}\text { Annunciator }(s): & \text { Priority } & \text { Alarm color (actual) } \\ \text { Hi: } & 2 & \text { IELCON } \\ \text { IO: } & 0 & \end{array}$

Other:

MrE Instr. No: $8 / 7-3.5-40-031$

Due Date: $10-22-910$ 
Tag: PDIS-AY102WF-2

P\&ID : H-2-131068, SH 1

LOOP: H-2-131333, SH 17

Manufacturer: DiNeR Mode1 No.: $213220 \mathrm{C}$ Serial No:

MTE Instr. No: $817-35.10-2.5$ Due Date: $8-8358$

Input Range $0-20$

Tolerance $5 \%$

Units: PSI

$\begin{array}{lc}\text { Input } & \text { Output } \\ 0 & 5 \\ 5 & 5 \\ 10 & 15 \\ 15 & 20 \\ 20 & .5\end{array}$

\begin{tabular}{|c|c|c|c|c|}
\hline $\begin{array}{l}\text { Lo: } \\
1.0 \\
\end{array}$ & $\begin{array}{l}\text { Hi: } \\
1,0 \\
\end{array}$ & $\begin{array}{c}\text { As Found } \\
.5 \\
\end{array}$ & $\begin{array}{l}\text { In/Out } \\
\mathrm{O}, \mathrm{i}\end{array}$ & $\begin{array}{c}\text { As Left } \\
\mathrm{O}\end{array}$ \\
\hline$\dot{H C}$ & 6.0 & 6.0 & not & 4.75 \\
\hline $9 . c^{-}$ & 11.0 & 11.5 & $0.1=$ & 10 \\
\hline 14.0 & 16.0 & 17.0 & הט & 15.25 \\
\hline 100 & 21.0 & 22.0 & OUT & 200 \\
\hline
\end{tabular}

(signoff)Calibrated By:

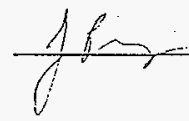

Date: $91-27$

Location: AYtoz Gordenser

\section{Comments}

SET SWITCH TO ALARM AT 15PSI, FUNCTIONAL TEST: DOES ALARM COME IN AT MICON? YES NO $X$

$$
\begin{aligned}
& \text { Not WIREOUP. } \\
& \text { JPerris }
\end{aligned}
$$


Tag: PDIS-AY102WF-2

P\&ID: H-2-131068, SH I

Mode1 No.:
LOOP: H-2-131333, SH 17

Sexial No:

MIE Instr. No: $817-35-40-031$

Due Date:

Input Range $0-20$

Tolerance $5 \%$

Units: PSI

Input output

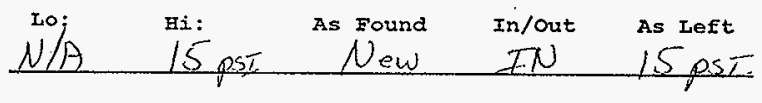

(signoff)calibrated By: Rig 2 Sat Hursen

Location:

Comments

SET SWITCH TO ALARM AT 15PSID

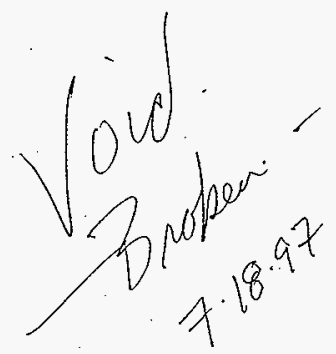


$5 / 14 / 96$

W-030 LOOP TEST DATA SHEET

HNF-SD-W030-TD-003,

REV. 0, PAGE 173

Tag: II_AY2_EWP_1A I/O TYPe: AI Description: EWPump1ACurxent

Cntlx: 46 Chan No: 25 P\&ID: H-2-131068 Logic: Y404

Remarks :

Location:

Actuation Method: OPERATE motor a mEASURE RUNAWh CURRENT

sensor calibration: $N / A$ - TRANSmITTER FACTOMY CALIBRATEO

$\square 5 \mathrm{pt}$ call

per procedure (data attached)

(signoff)

LOOP Tolerance:

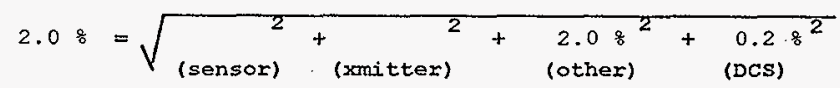

Units: AMP

Process Input

Readout Tolerance

Lo: $\quad 0.00$

Bi:-20.00-19.60 $20.40=$

Running) current 9.4

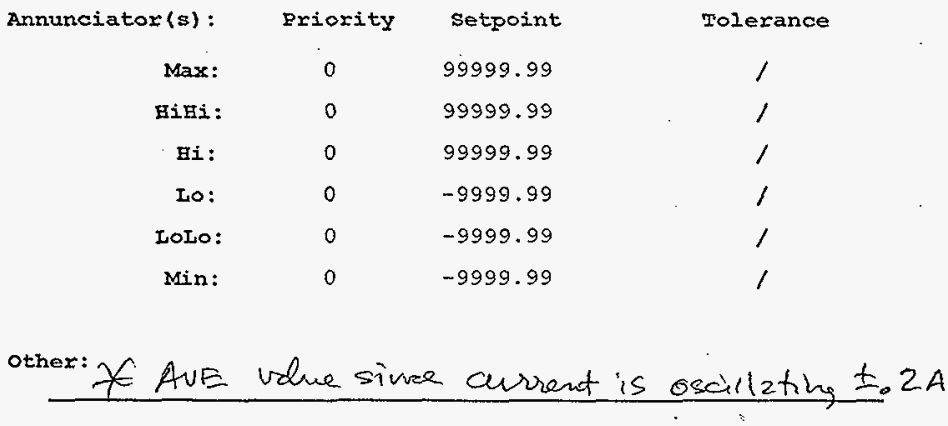

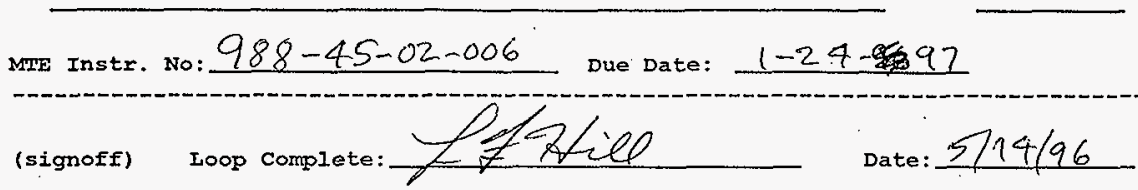

MTE Instr. No: $\frac{988-45-02-006}{1-24-497}$ Due Date: $\frac{1-29}{\text { Loop complete: }}$ Date: $5 / 14 / 96$

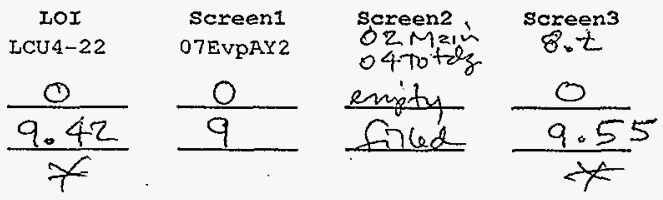

Trip Point

$\bar{\square}$


$5 / 14 / 97$

W-030 CALIBRATION DATA SHEET

HNF-SD-W030-TD-003,

REV. 0, PAGE 174

Tag: TT-AY102EWP-1A

P\&ID : $\mathrm{H}-2-131068$, SH 1

LOOP: $H-2-131333, \mathrm{SH} 6$

Manufacturer: KATY INSTRS Model No. 420 Serial No:

MTE Instr. No: $817.45 .02 \cdot 004$

Due Date: $\begin{aligned} & 5 \cdot 16 \cdot 92 \\ & z \cdot 28 \cdot 98\end{aligned}$

Input Range $0-20$

Tolerance $2 \%$ - screen

Units: Amps

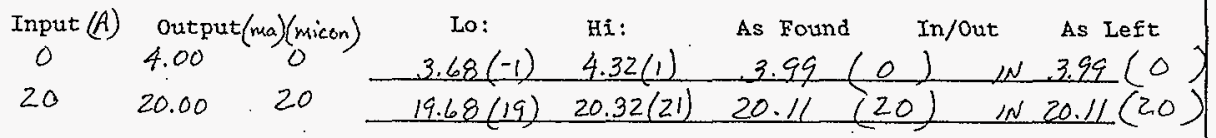

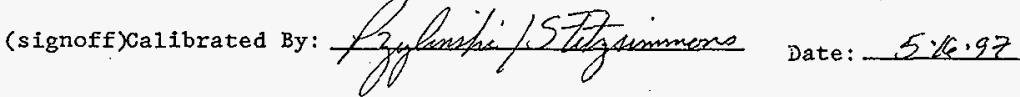

Location: $102 A Y$ CoOlinG WATER Tower (Pun pIN)

comments factory calibrated; check zero; SPAN. Use current generator w/ calibrated MTE clamp on meter 
Tag: II_AY2_EWP_1B I/O TYPe: AI

Cntlr: 46

Remarks :

Location:

Actuation Method: Gperite motor As measure vumisy quivent

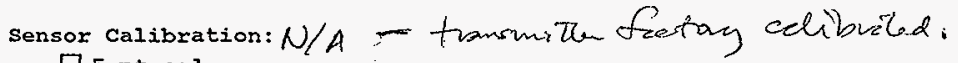
$\square 5$ pt cal pet procedure (data attached)

Loop Tolerance: $\quad 2.0 \div=\sqrt{\text { (sensor) }{ }_{\text {(xmitter) }}^{2}+\text { (other) }^{2}+0^{2}+0_{\text {(DCs) }}^{0.22^{2}}}$

Units: $A M P$

Process Input

Lo: $\quad 0.00$

II: 20.00 tuming currint 9.25

Annunciator(s):

Max:

HiEi :

Ei:

LO:

LOIO:

Min:
Readout Tolerance

$-.40 \%$

.40 $19.60 / 20.40$ $8.82 / 9.65$

Priority

setpoint 99999.99

99999.99

99999.99

$-9999.99$

$-9999.99$

$-9999.99$

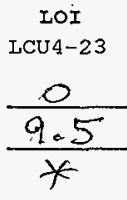

Tolexance

l

/

1

I

/

I
Screen1
07EvpAY2 \& 0 4 Totalz

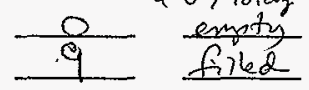

Trip Point

other

A AUE udve since current is oscillating. I. $2 \mathrm{~A}$

MTE Instr. No: $988-45-02-006$ Due Date: $1-24-97$ 
Manufacturer: KATY INSTRS. Mode1 No.: 420 Serial No:

\section{$817.45 \cdot 02 \cdot 004$}

MTE Instr. No: $8 / 7 \cdot 45 \cdot 08 \cdot 040$

Due Date: $\begin{array}{r}5 \cdot 16 \cdot 97 \\ 2-28 \cdot 98 \\ \hline\end{array}$

Input Range $\Delta-20$.

$5 \%$ screen

Units: Amps

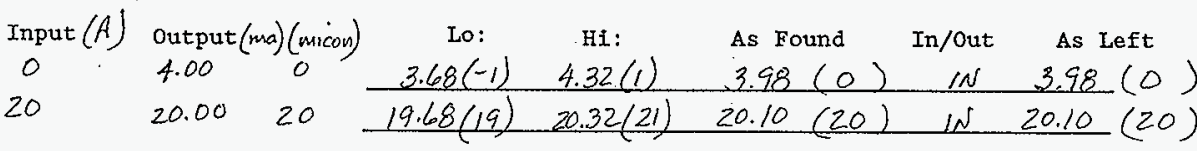

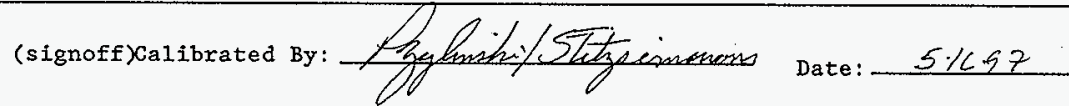

Location: 102AY CoOLNG WATER Tower (PunsiB)

comments Factory calibrated; check zero; span. Use current generator w/calibrated MTE clamp on meter 
Tag: IAL_AY2_EWTK_1 I/O TYPe: DI

Description: AY2EWTwrLvlLo

CntIr:

48 Chan No:

23

PEID: $H-2-131068$

Logic:

Remarks :

Location:

Actuation Method: screwdiven to zefunte buel switzl thre test

$$
\text { port provided. }
$$

Sensor Calibration:

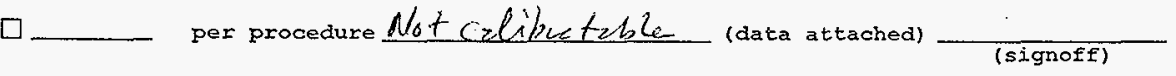

Setpoint:

Loop Tolerance:

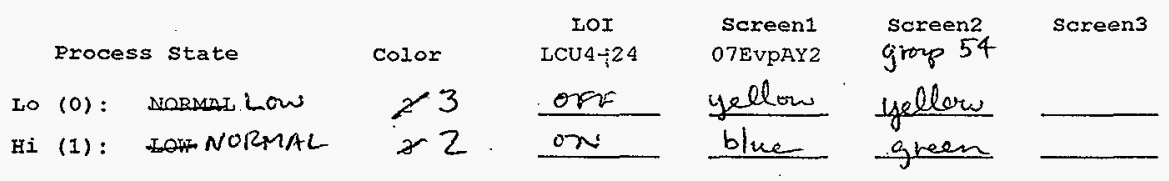

$$
\begin{aligned}
& \text { Annunciator(s): Priority Alarm Color (actual) }
\end{aligned}
$$

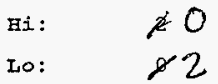

$$
\begin{aligned}
& \text { tott } \\
& \frac{\text { yellow }}{\text { yen }}
\end{aligned}
$$

revised pen KSt. divection $19 t$

Other:

MIE Instr. No:

Due Date:

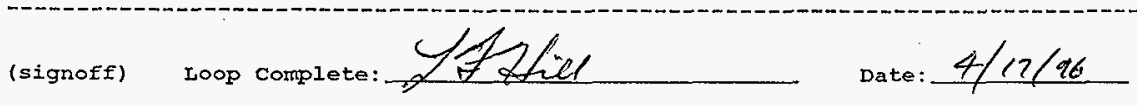


Tag: LSL-AY102EUTK-1

P\&ID: H-2-131068, SH 1

LOOP: $\quad H-2-131333$, SH 16

Manufacturer: $\quad \frac{\text { ITTMCD RNWEL }}{i \text { MILER }}$ Model No.:

$63 M$ Serial No:

$2 E \cdot 000-732$

MTE Instr. No:

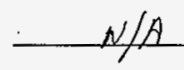

Due Date:

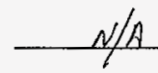

Input Range Functional test

Toierance $\omega / A$

Units: Sw/7CHA

Input Output Lo: Hi: As Found In/Out As Left

$M E C H$

TRIP SWITCH

"SEE WSTRUCTIONS IN COMMENTS

(signoff) Calibrated By:

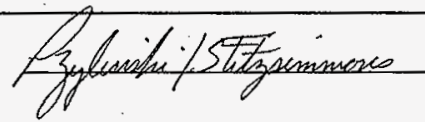

Date: $\quad 4 \cdot 18 \cdot 97$

Location: HOZAY COOWNG WATER TOWER.

Comments

Instructions: Actuate level switch with screwdriver through access port provided. Verify LAL (YELLow) recieved on MICON screen.

IS ALARM RECEIVED AT MICON? YYN 
Remarks :

\section{Location:}

Actuation Method: TUR,ED LEPKi PETECrEM QP/OFF

sensor calibration: $i / / \mathcal{H}$

$\square$ per procedure (data attached)

Setpoint:

Loop Tolerance:

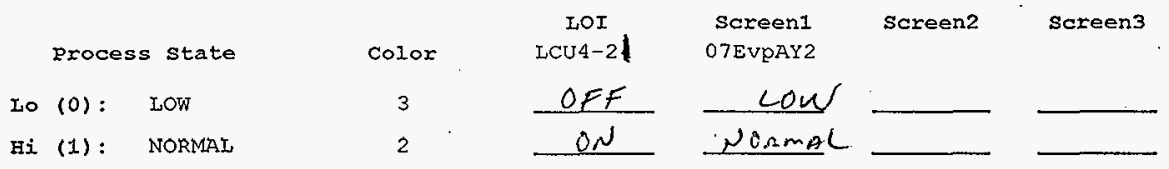

Annunciator(s): Priority

Hi: 0

Lo: 2
Alarm color (actual)

YEciou)

other:

MIP Instr. No:

Due Date: 
i: CE/CSH-AZ102EWT-1 1/0 Type: N/A Description: Tower Cond.

P\&ID: $H-2-131070$

Remarks: Local 100p on A2102 Cooling Tower with no input to the MCS. Controls conductivity of the tower water via feed and bleed.

Location: Conductivity element in cooling tower filtration ioop. Conductivity switch/controller located in panel next to local tower control panel

Buffer solution

Actuation Method: Simulate conductivity changes with a decade-box and verify that the controller will open the tower drain valve on high conductivity and close the drain valve when conductivity is below setpoint $(1000 \mu$ mho)

Comments: Peure pesfonmed to changs surtich from 120 VAC to I2VAC.

MTE Instr. No: Buffes Solse S/N7070 Due Date: $2 / 98$ (signoff) Loop Complete:

Date: $6 \cdot 12 \cdot 90$ 
$6 / 4 / 96$

W-030 CALIBRATION DATA SHEET

HNF-SD-W030-TD-003,

REV. O, PAGE I81

Tag: CSH-AZ102EUT-1

P\&ID: $H-2-13107067$, SH 1

LOOP: W-030-P1

Manufacturer: IDEX

Model No.: SHICEFACX

Serial No: $-5 F \cdot 727$

MTE Instr. No: Buffer Sold. S/N 7070 Due Date: $2 / 98$

limited rial.

Input Range $0-6,000$

Units: $\mu$ mhos / cm

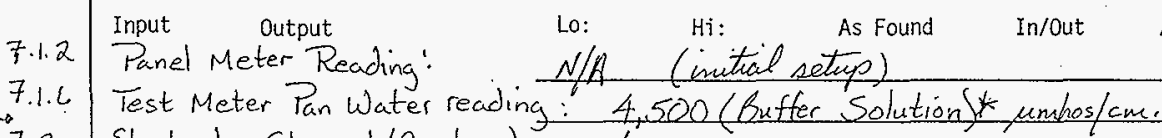

7.2 Electrode Cleaned/Replaced : No

7.3 Specified Trip Point Setting. 1000 un shes /om

7.3.2 As found Trip Point Panel meter reading. N/A. (initial setup.)

7.4.2 Control indicator Light Panel Meter reading 1,020 , umbos/am

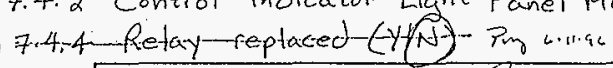

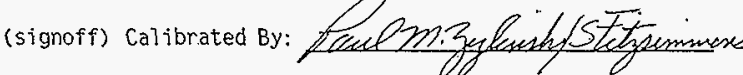

Date: $6 \cdot 46 \cdot 96$

Location: Az Cooling Water Towers
Tolerance $2 \%$

In/ Out As Left 
ר̣: FSL-AY102WS-1 I/0 Type: N/A

Description: Tower Cond. Flow switch

P\&ID:H-2-131068

Remarks: Local 100p with no inputs to the MCS. Interupts conductivity controls if there is no flow established through the conductivity cell.

Location: Flow switch located at AY102 cooling tower in filtration loop downstream of conductivity element.

Actuation Method:

Actuate switch 7ocally or simulate switch actuation using electrical jumper. Verify that a no flow condition prevents the conductivity cell from acutating the tower drain valve on high conductivity.

Comments:

MTE Instr. No:

(signoff) Loop Complete:
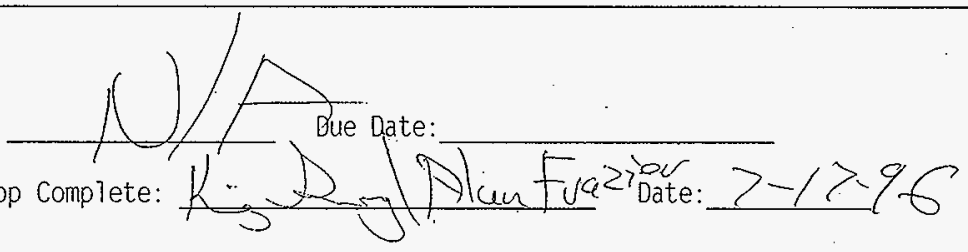
Tag: FSL-AY102WS-1

P\&ID: $\quad H-2-131068$, SH 1

LOOP:

Manufacturer: Dex/Pulsafeeder Model No.: Qutuor, 3

Serial No: SF-726

MTE Instr. No:

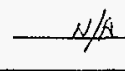
Due Date: Funstronal

Units:

Input

output

Lo:

Hi:

As Found

In/out

As Left

Functional

(signoff) Calibrated By:

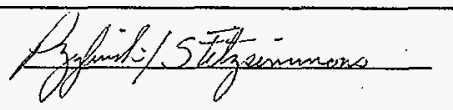

Date:

$5.28 \cdot 97$

Location:

102 AY COOLWG WATER YOWEE.

Comments * De-energize circ pump to cause low flow.

Does flow light on Conductivity meter (CSH-AY102EWT-1) light up when flow switch is activated? $(9) N$

* Manually 
TC

Tag: TS-AY102EWT-1A2
P\&ID : H-2-131068, SH 1
LOOP:

Manufacturex: Joknson Controlsmodel No.: ABфABA-44 serial No: $2 E-000-702$ $817-13.55 .025$

MTE Instr. No: $\frac{817 \cdot 13 \cdot 55-014}{817-45-08-044}$ $7-24-97$

Due Date: $\frac{11-18-97}{2-12-98}$
Input Range 60-140 Tolexance NAR $519 \%$

Units: DEG F

Input Output $100^{\circ} \mathrm{F}$ switch
Lo:

98.4
Hi:

As Found 100.1

In/Out

As Left ZW 100.1

(signoff)Galibrated By: Fifasimanons/Zylinst, Date: $4-25-97$

Location: 102AY Cooling Water Tower

Comments

CALIBRATE PER VENDOR DATA SET TO TURN ON AT 100 DEG F 
LIMITED

$3 / 8 / 96$

W. $030^{\vee}$ CALIBRATION DATA SHEET

HNF-SD-W030-TD-003, TS.

P\&IO: H-2-131068, SH 1

REV. 0, PAGE 185

Tag: IE-AY102EWT-1AL

P\&IO: H-2-131068, SH 1 LOOP:

Manufacturer: Johnson Controls model No.: A19ANC-25 serial no: $2 E-000-698$

$8 / 7 \cdot 45 \cdot 08-040$

$2 \cdot 5 \cdot 97$

MTE Instr. No: $\frac{778-13-55-002}{2}$ Due Date: $\frac{m_{32}^{2}}{3 \cdot 9-19 \cdot 96}$

Input Range $0-150$

Units: DEG F

Input. Output

$40^{\circ} \mathrm{F} \quad 40^{\circ} \mathrm{F}$ (SWiTCH)
Tolerance N/A $2 \%$

\begin{tabular}{lllll} 
Lo: & $\mathrm{Hi}$ & As Found & In/Out & As Left \\
$37^{\circ} \mathrm{F}$ & $43^{\circ} \mathrm{F}$ & $\mathrm{N} / \mathrm{A}$ & $\mathrm{N} / \mathrm{A}$ & $40.0^{\circ} \mathrm{F}$ \\
\hline
\end{tabular}

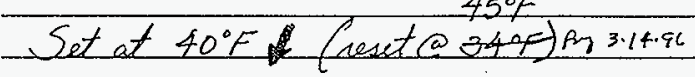

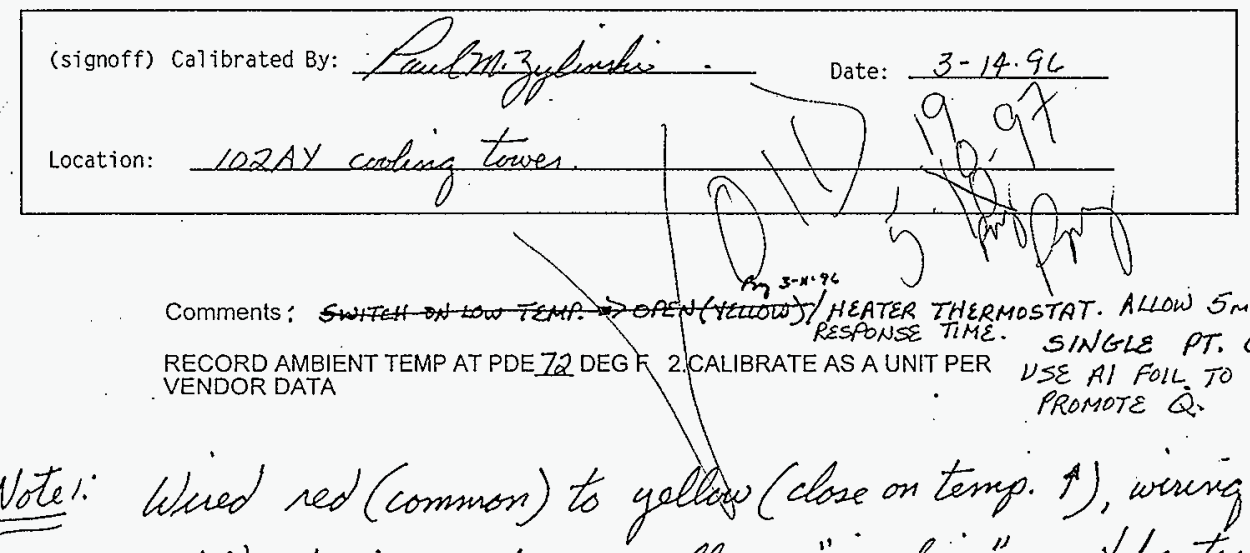
(electrical) diagram show yellow - "in line" - w/ heater element. - Please check to make sure this isis opposite desired position!!!

Noted Response time required due to long length of capillary tide. (min $=5$ mix.) 
Tag: TS-AY102EWT-1A1

P\&ID : H-2-131068, SH 1

LOOP :

Manufacturex: Johnson Control Model No.: A19ANC-25 Serial No: 2E-000-698 MTE Instr. No: $\begin{array}{r}817-13 \cdot 55 \cdot 0.25 \\ 817-13 \cdot 55-014\end{array}$ Due Date: $\frac{\begin{array}{l}7-24-97 \\ 11-18-97\end{array}}{2-12.98}$

MTE Instr. No: $\frac{817.45-08-044}{817}$

Input Range 0-150

Tolerance N/A

Units : $D E G F$

\begin{tabular}{|c|c|c|c|c|c|c|}
\hline $\begin{array}{l}\text { Input } \\
40^{\circ} \mathrm{F}\end{array}$ & $\begin{array}{l}\text { Output } \\
40^{\circ} F \text { (Switch) }\end{array}$ & $\begin{array}{l}\text { Lo: } \\
37^{\circ} F\end{array}$ & $\begin{array}{l}\text { Hi: } \\
43^{\circ} \mathrm{F}\end{array}$ & $\begin{array}{c}\text { As Found } \\
45.1\end{array}$ & $\begin{array}{l}\text { In/Out } \\
\text { ouT }\end{array}$ & $\begin{array}{l}\text { As Left } \\
39.8\end{array}$ \\
\hline . & & \multicolumn{5}{|c|}{ Set at $40^{\circ} \mathrm{F} \downarrow$ (reset at $45^{\circ} \mathrm{F}$ ) } \\
\hline
\end{tabular}

(signoff)Galibrated By: Fitasimmons

Date: $3-14-97$

Location: 1024 Coling Tower

Comments

CALIBRATE PER VENDOR DATA SET TO TURN ON AT 40 DEG F 
Tag: FI_AZ1_EWR_1 I/O Type: AI Description: EwrHdr Flow
Cntlr: 51
Chan No:
10
P\&ID : $\mathrm{H}-2-131069$
Logic:

Remarks :

Location:

Actuation Method: Betz Colibator on $x$ motter isput

Sensor Calibration:

$\otimes^{\prime} 5$ pt cal per procedure $V I d z \hbar$ (data attached)

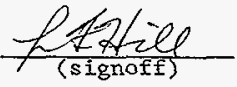

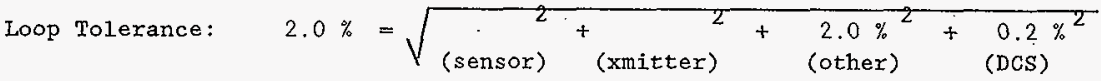

Units: GPM

Process Input

Readout Tolexance

Lor

Lo: $\quad 0.00$

Hi: $\quad 400.00$

$$
-8.04-8.04
$$

$391.96-408.04$

$\begin{array}{rrr}\text { Screen1 } & \text { Screen2 } \\ \text { LcU3-16 } & \text { 08EvpAZ1 } & \text { gremp } 62\end{array}$

Screen 3

$\frac{-0.56}{399.28} \frac{-0.40}{400.00 .02}$

$\begin{array}{rccc}\text { Annunciator(s): } & \text { Priority } & \text { Setpoint } & \text { Tolerance } \\ \text { Max: } & 0 & 99999.99 & - \\ \text { HiHi: } & 0 & 99999.99 & - \\ \text { Hi: } & 0 & 99999.99 & - \\ \text { Lo: } & 2 & 295.00 & 286.96-303.04 \\ \text { LoLO: } & 0 & -9999.99 & - \\ \text { Min: } & 0 & -9999.99 & -\end{array}$

Trip Point

Other:

totalizer weiventad

MTE Instr. No: Bef2 Celbutor

Due Date: $817-23-01-005$ 
$5 / 1 / 97$

W-030 CALIBRATION DATA SHEET

HNF-SD-W030-TD-003,

REV. 0, PAGE 188

Tag: FT-AZ101EWR-1

P\&ID: H-2-131069, SH 1

LOOP: H-2-131334, SH 11

Manufacturer: Fisher Porter Model No.: $50 L V-3212 B 1 J B$ Serial No:

$817 \cdot 23 \cdot 01 \cdot 015$

$93 \omega 017046$

MTE Instr. No: $\quad 817.45 .08 \cdot 040$.

6.4 .97

Due Date: $\quad 2 \cdot 28 \cdot 98$

Input Range $0-400(0-64 \mathrm{~Hz})$

Tolerance $2 \%$

Units: gem

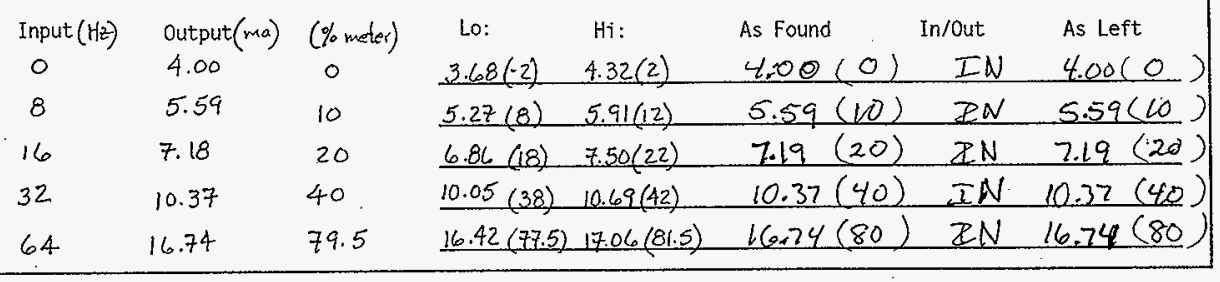

(signoff) calibrated By: Fitzsimmuns/Zyloingki $\quad$ Date: $5-16-97$

Location: $101-A z$ Cooling Water Tower

comments Calibration factor $(\bar{K})=24.115 \mathrm{C} / \mathrm{gal} @ 93^{\circ} \mathrm{F}$

$f_{0}=160.8 \mathrm{~Hz}$

Span factor $=1083$

$f_{\text {test }}=80.4 \mathrm{~Hz}(\max )$

reified: Span readings at MAcoN. Pry

$$
\begin{aligned}
& 0=0 \text { gp } \\
& 80 \%(320 \mathrm{gpm})=320 \mathrm{gpm}
\end{aligned}
$$


$6 / 11 / 96$

W-030 CALIBRATION DATA SKEET

HNF-SD-W030-TD-003,

REV. O, PAGE 189

Tag: FT-AZ101EWR-1

REID: $\mathrm{H}-2-131069$, SH 1

LOOP: H-2-131334, SH 11

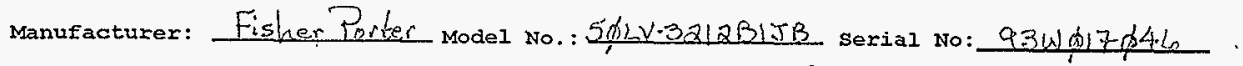

MTE Instr. No: $\begin{aligned} & 817 \cdot 45 \cdot 08-040 \\ & 817-23-01-015\end{aligned}$

$=\frac{2 \cdot 12 \cdot 95}{6-4 \cdot 97} 2-5-97$

Input Range $0-400$

Tolerance $2 \%$

Units: gem

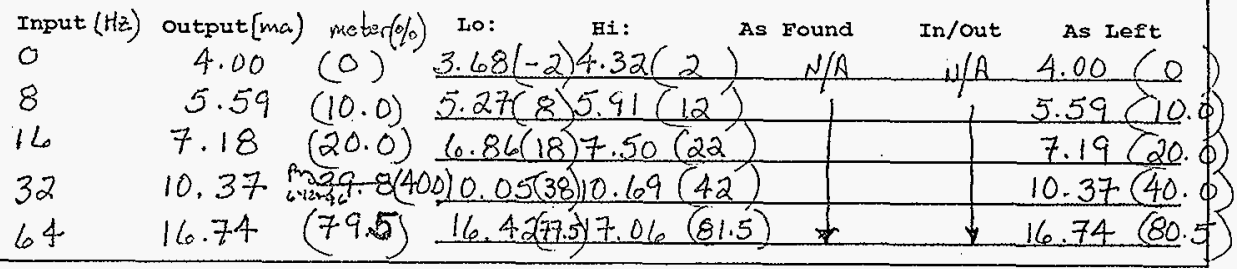

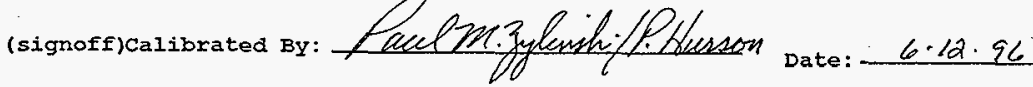

Location: $10 L-A Z$ COO/ ING LATER TOWER.

comments Calibration factor $(\vec{k})=24.115 \mathrm{c} / \mathrm{gal} @ 93^{\circ} \mathrm{F}$

$f_{0}=160.8 \mathrm{~Hz}$

Span factor $=1083$

$f_{\text {rest }}=80.4 \mathrm{~Hz}$ (max)

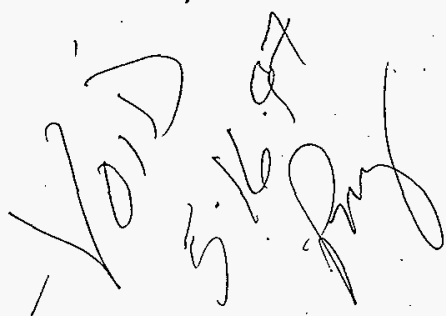




\section{Tag: PDAH_AZ1WF_2 I/O TYPe: DI Description: AZ1EWFtrDpHi}
Cntix:
52 Chan No: 20
P\&ID: $\quad$ H-2-131069
Logic:

Remarks :

Iocation:

Aatuation Method: REGULATEA PRESSURE SOURCE

Sensor Calibration:

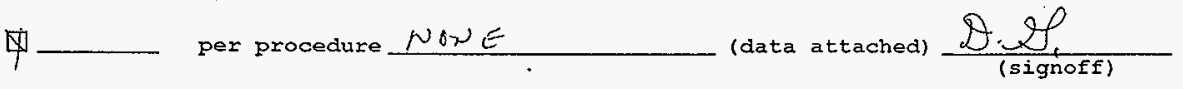

Setpoint: $\quad 15 \mathrm{psi}$

Loop Tolerance:

\begin{tabular}{|c|c|c|c|c|c|c|c|}
\hline \multirow{2}{*}{\multicolumn{2}{|c|}{ Process }} & & & LOI & Screen 1 & Screen2 & Screen 3 \\
\hline & & state & color & LCU3-15 & 08EvpAz1 & & \\
\hline Lo & $(0):$ & NORMAL & 2 & & & & \\
\hline Hi & (1): & HIGH & 3 & & & & . \\
\hline
\end{tabular}

$\begin{array}{rcc}\text { Annunciator(s): } & \text { Priority } & \text { Alarm Color (actual) } \\ \text { Hi: } & 2 & \\ \text { IO: } & 0 & \end{array}$

Other:

MTE Instr. No: $817-35-40-031$ Due Date:

(signoff) Loop complete: 0.4 
Tag： PDIS-AZ101WF-2

Manufacturer: DWYER

\section{$H \cdot 2 * 310695 H 1$}

P\&ID : $-030-P 1-P_{\text {rz }} 7 \cdot 18.97$

Model No.: $43220 \mathrm{C}$
LOOP: H-2-131334, SH 17

Serial No:

$\begin{array}{ll}\text { Input Range } 0-20 \\ \text { Units: PSI } \\ \text { Input } & \text { Output } \\ 0 & 0.00 \\ 5 & 5.00 \\ 10 & 10.00 \\ 15 & 15.00 \\ 20 & 20.00\end{array}$

Input Range $0-20$

Tolerance $2.00 \%$

MTE Instr. No: $817-35-40-031$

Due Date: $7 \cdot 31 \cdot 97$

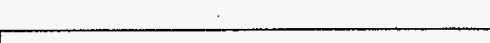

Units: PSI

$2 E-000-7129$.

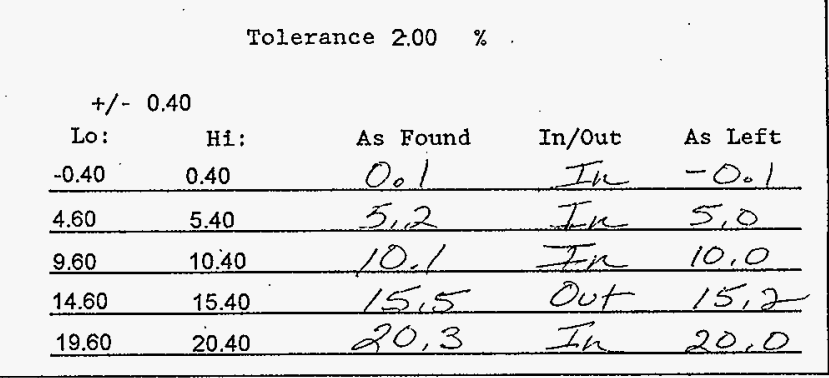

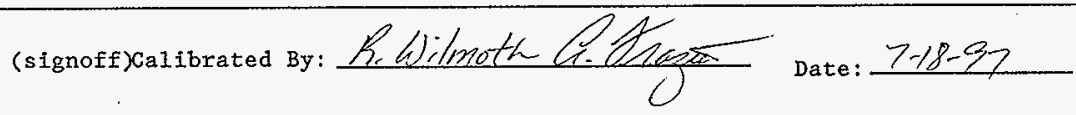

Location: AZIO1 CONAER

Comments

SET SWITCH TO ALARM AT 15PSI, FUNCTIONAL TEST: DOES ALARM COME IN AT MICON? YES NO 2

Power not available, limited cal. 
Manufacturer:

Model No.:

Serial No:

MIIE Instr. No: $817-35-40-031$ Due Date:

Input Range $0-20$

Units: PSI

Input output

$15 \mathrm{psi}$ switch
Tolerance $5 \%$

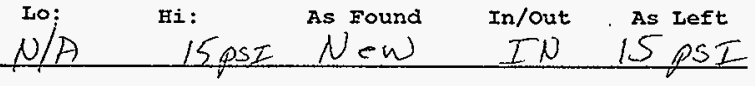

(signoff)calibrated By: $\frac{1}{3} 3$ ) Pat Hwison Date: $4-16-96$

Iocation:

Comments

SET SWITCH TO ALARMAT 15PSID

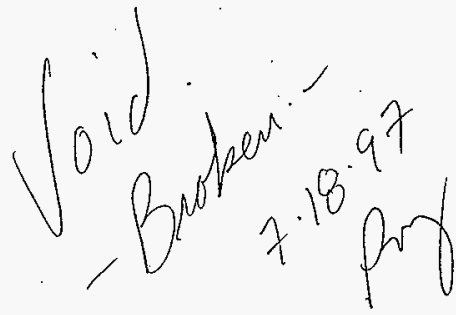


Tag: II_AZ1_EWP_1A I/O Type: AI

Cntlr: 51 Chan No: 11
Description: EwPumplACurrent

P\&ID: H-2-131069
Logic: $Y 408$

Remarks :

Location

Actuation Method: CORRENT SOURCE tHROVGH CT WRITH CALAR ATED

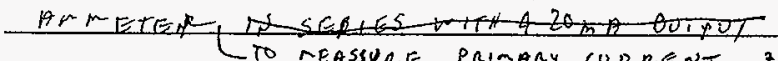

Sensor Galibration: $31 A$

$\square 5$ pt cal

Witl Loof per procedure (data attached)

(signoff)

Loop Tolerance: $\quad 2.0 \%=\sqrt{{ }_{(\text {sensor) }}^{2}+{ }_{\text {(xmitter) }}^{2}+\text { (other) }_{\text {(on }}^{2.0 \%}{ }^{2}+0.2 \%^{2}}$

Units: AMP

Process Input

Lo: $\quad 0.00$

Hi: $\quad 50.00$
Readout Tolerance

$-1.00-1.00$

$49.00-51.00$
IOT

LCU $3-16$

$-0.03$

int.

49.53
Screen1 08EvpAZ1

$O$

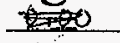

50
Screen2 c2msin

$Q P E$ $O x$
Screen 3 04 total

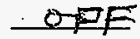

$\sigma x$
Tolerance

Trip Point

$\begin{array}{rrrrr}\text { Max: } & 0 & 99999.99 & - & \\ \text { HiHi: } & 0 & 99999.99 & - & \\ \text { Hi: } & 0 & 0.00 & - & \\ \text { Lo: } & 0 & -9999.99 & - & - \\ \text { LoLo: } & 0 & -9999.99 & - & \\ \text { Min: } & 0 & -9999.99 & & \end{array}$

Other:

$817-45-02-010$

MTE Instr. No: $817-45-08055$

$1 / 24 / 42$ Due Date: Htos 
Tag: IT-AZ101EWP-1A

Manufacturex: KATY NSTRS. Model No. : $A 20$ Serial No:

\section{$817 \cdot 45 \cdot 08 \cdot 040$}

MTE Instr. No: $817 \cdot 45 \cdot 02 \cdot 004$
$2 \cdot 28 \cdot 98$

Due Date: $5 \cdot 16 \cdot 97$

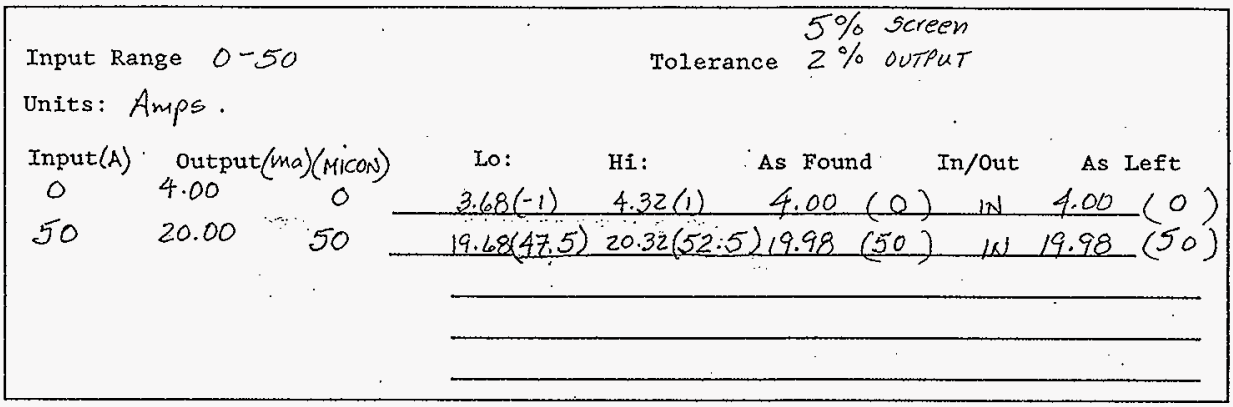

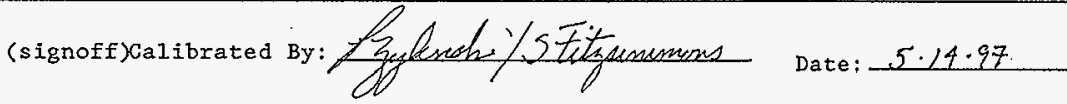

Location: IOIAZ COOLNG WRTER TOWER.(PUMP iA)

comments Functional, Factory calibrated; check zero and span. Use current generator w/ calibrated (MTE) clamponmeter 
Tag: II_AZ1_EWP_1B I/0 Type: AI

Cnt1r: 51 Chan No: 12
Description: EwPumplBCurrent

P\&ID : H-2 - 131069
Logic: $Y 410$

Remarks :

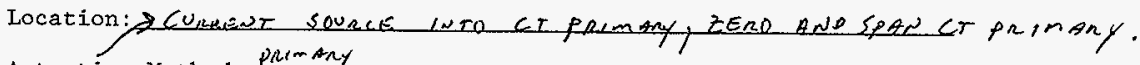

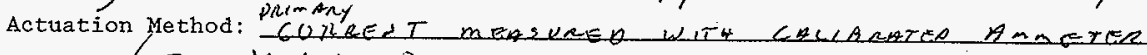
(

Sensor Galibration:

$\square$ pt cal
$\rrbracket$ Wit coop pex procedure (data attached)

Loop Tolerance:

$$
2.0 \%=\sqrt{{ }_{\text {(sensor) }}^{2}+{ }_{\text {(xmitter) }}^{2}+\begin{array}{c}
2.0 \% \\
\text { (other) }
\end{array}^{2}+\begin{array}{c}
0.2 \%^{2} \\
\text { (DCS) }
\end{array}}
$$

Units: AMP

Process Input

Lo: $\quad 0.00$

Hi: $\quad(50.00) \nsucc$

$(50.00) \neq \quad 49.00-51.00$
F $20 \mathrm{~mA}$ zppleed for input eleck

Annunciator(s): Priority Setpoint

eadout Tolerance

$$
-1.00-1.00
$$

$\begin{array}{ll}\text { Max: } & 0 \\ \text { HiHi : } & 0\end{array}$

Hi: $\quad 0$

Lo: 0

LoLo: 0

Min:

0
99999.99

99999.99

0.00

$-9999.99$

$-9999.99$

$-9999.99$

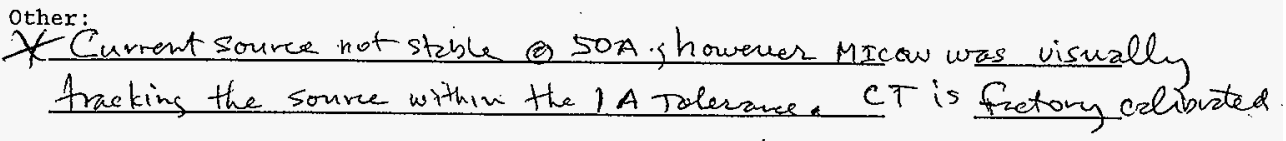
MTE Instr. No: 817-45-02-010 Due Date: $1 / 24 / 97$

LOI LCU3 - 17

Screen 1 08EvpAZ1

Screen2 ozMain Screen
o4totelg $\frac{0.00}{49.92}$ $\frac{0}{50}$ $\frac{\text { OTFF }}{\text { ON }}$ OPE ON $\rightarrow$

Tolerance

Trip Point 
$4 / 25 / 97$

W-030 CALIBRATION DATA SHEET

$$
\begin{aligned}
& \text { HNF-SD-W030-TD-003, } \\
& \text { REV. 0, PAGE } 196
\end{aligned}
$$

Tag: IT-AZ101EWP-1B

P\&ID: H-2-131069, SH 1

LOOP: $\mathrm{H}-2-131334$, SH 5

Manufacturer: KATY iNSTR. Model No.: 420

Serial No:

$817 \cdot 45 \cdot 08 \cdot 040$

MTE Instr. No: $817 \cdot 45 \cdot 02 \cdot 004$

Due Date: $\begin{array}{r}2 \cdot 28 \cdot 98 \\ 5 \cdot 16 \cdot 97\end{array}$

Input Range $0-50$

Units: Amps

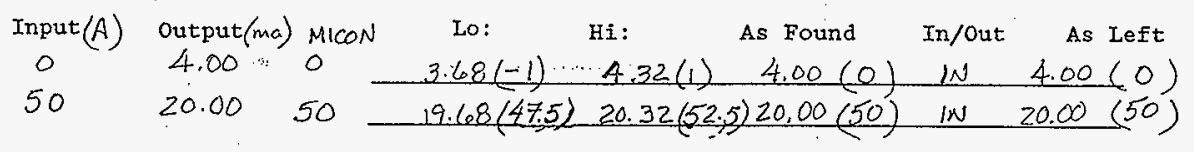

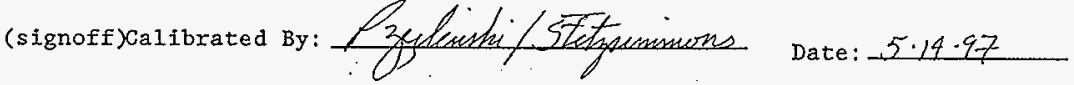

Location: $10 / A Z$ COOLIE WATER TOWER (PUMP IB)

comments. Functional, Factory calibrated; check zero and span Use current generator w/ calibrated (MTE) clampon meter. 
Tag: LAL_AZI_EWTK_1. I/O TYpe: DI 52 Chan No: 23
Description: Az1EWTwrLvlLo

P\&ID: $H-2-131069$
Iogic:

\section{Remarks:}

Location:

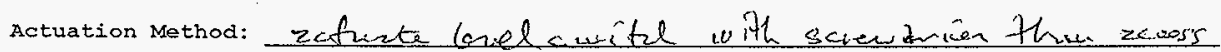
port pwôndet

Sensor Calibration:

[ . per procedure Not celikntabls (data attached)

Setpoint:

Loop Tolerance:

Process state

Io $(0)$ : HOW LOW

Hi (1): IOFINORARAL
LOI

color

23

32 on screen1 O8Evpaz 1 LCU3-18

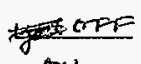

yellow:

blue
Screen2 group 64 yellow grean
Annunciator (s):

Hi:

Io:

Priority
20
.02

Alarm color (actual)

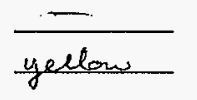

revised direction
pert

other:

MTE Instr. No:

Due Date: 
2/19/97

W-030 CALIBRATION DATA SHEET

HNF-SD-W030-TD-003,

REV. O, PAGE 198

Tag: LSL-AZ101EWTK-1

P\&1D: H-2-131069, SH 1

LOOP: H-2-131334, SH 16

Manufacturer: $\frac{\text { IIT McDonnell Model No.: }}{\dot{i} M \text { Miller }}$ le 3M
MTE Instr. No: $\quad-M / A$

Serial No: $\quad 2 E-000 \cdot 733$

Input Range Functional test

Tolerance $N / A$

Units: Switch

Input Output

Lo:

$\mathrm{Hi}$ : As Found

In/Out

As Left

Mech. Suited

"SeE INSTRUCTIONS UN COMMENTS"

(signoff) Calibrated By:

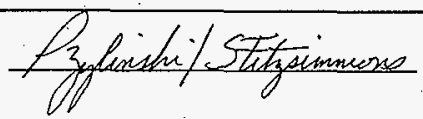

Date: $4 \cdot 18 \cdot 97$

Location: IOI AZ COOLWG WATER TOWER.

Comments

Instructions: Actuate level switch with screwdriver through access port provided. Verify LAL (Yellow) recieved on MACoN screen.

IS RLARM REceived at MICON Y Y 
June 4. 1996

W030 LOOP TEST DATA SHEET

$$
\begin{aligned}
& \text { HNF-SD-W030-TD-003, } \\
& \text { REV. O, PAGE } 199
\end{aligned}
$$

7G: CE/CSH-AZ101EWT-1 I/O Type: N/A

Description: Tower Cons.

r\&ID: $H-2-131069$

Remarks: Local loop on AZ101 Cooling Tower with no input to the MCS. Controls conductivity of the tower water via feed and bleed.

Location: Conductivity element in cooling tower filtration loop. Conductivity switch/controller located in panel next to local tower control panel

Actuation Method: Simulate conductivity changes with a decade box and verify that the controller will open the tower drain valve on high conductivity and close the drain valve when conductivity is below setpoint (1000 mho)

Comments:

Censure performed to change acietch grover $120 \mathrm{VAC}$ to $12 \mathrm{VAC}$

MTE Instr. No: Buffer sem soN fo 70 Due Date: $2 / 98$

(signoff). Loop Complete:

trambenzyliosich

Date: 6e:12.90 
$6 / 4 / 96$

W.030 CALIBRATION DATA SHEET

HNF-SD-W030-TD-003,

REV. 0, PAGE 200

Tag: CSH-AZ101EWT-1

P\&ID: $H-2-131069$. SH 1

LOOP: W-030-P1

Manufacturer: TDEX

Model No.: SHLCEZACX Serial No: SF .725

MTE Instr. No: Buffer sole. 7070 Due Date: 2-98

Limiter) cal

Input Range $0 \cdot 6,000$

Units: remho's/cm
Tolerance $2 \%$

Lo: Hi: As Found

In/Out

As left

1.2 Panel Meter Reading: N/A -initial cal

1.6. Test Meter Pan Water reading: * 4500 unishoslcm

.2 Electrode Cleaned/Replaced : No

3 Specified Trip Point Setting: $1000 \mu$ mhos $/ \mathrm{cm}$

3.2 As found Trip Point Panel meter reading: N/A (initial setup)

4.2 Control indicator Light Panel Meter reading 1020 gumbos $/ \mathrm{cm}$ $\because 4.4-$ Seleverid replaced $(M / N) 6 \cdot 11-96$

(signoff) Calibrated By:

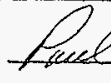

Date: $6 \cdot 13 \cdot 94$

Location: Az Cooling Water towers

7.1.8 Conductivity controller Panel reading 4,500 pushostam 7.1 .9 Is cond.meter and conductivity controller within $2 \%$ comments

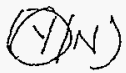

* Buffer sols. used while system stD: drained.

7.4 .5 Is the reading from 7.4 .2 within $6 \%$ of specified trip point setting? (IN) 
P\&ID:H-2-131069

Remarks: Local 100p with no inputs to the MCS. Interupts conductivity controls if there is no flow established through the conductivity cell

Location: Flow switch located at AZ101 cooling tower in filtration loop downstream of conduct ivity element.

Actuation Method:

Actuate switch locally or simulate switch actuation using electrical jumper. Verify that a no flow condition prevents the conductivity cell from acutating the tower drain valve on high conductivity.

Comments:

MTE Instr. No: Buffer selu S/N 7070 Due Date: (signoff) Loop Complete:

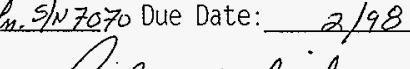
- Date: $6 \cdot 22 \cdot 96$ 
Tag: FSL-AZ101WS-1

LOOP:

Manufacturer: Idexpulscifedet Model no.: Oftion 3 Serial No:

SF 25

MTE Instr. No:

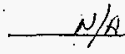

Due Date:

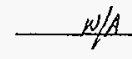

Input Range

Tolerance

Units:

Input Output

Lo:

$\mathrm{Hi}:$

As Found

In/Out

As Left

\section{Functional}

(signoff) Calibrated By:

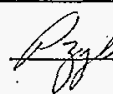

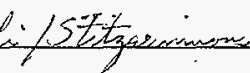

Date: 529.97

Location: 10LAZ Gosling Wates towes

Comments De-energize circ. pump to cause. low flow.

Does flow light on Conductivity meter(CSH-AZ1O(EWT-y) light up when flow switch is activated? $Y N$

* Manually activated switch while system is s/D and drained. 
Tag: TC $-A Z 101 E W T-1 A 2$

P\&ID : H-2-131069, SH 1

LOOP :

Manufacturer: $\frac{\text { Johnson Controlsmodel No. : ABQBABA-44 }}{817-13-55-025}$ Serial No: $\frac{2 E-000-203}{7-24-97}$ MTE Instr. No: $817-13-55.014$ $817-45-08-044$ Due Date: $\frac{11-18-97}{2-12-98}$

Input Range $60-140$ Tolerance $\operatorname{Pin}^{5 \cdot 14 \cdot \% 7} 2 \%$

Units: DEG $F$

Input Output Lo: $100^{\circ} \mathrm{F}$ Ssutch

\begin{tabular}{ccccc} 
Lo: & Hi: & As Found & In/Out & As Left \\
98.4 & 101.6 & 100.4 & FN & 100.4 \\
\hline
\end{tabular}

(signoff)Calibrated By: Fitzsimmons/Zylinskt Date: $\frac{4-15-97}{5-9}$ Location: 101 AZ Cooling Water Tower

Comments

CALIBRATE PER VENDOR DATA SET TO TURN ON AT 100 DEG F 
$6 / 3 / 96$

W-030 CALIBRATION DATA SHEET

HNF-SD-W030-TD-003,

REV. O, PAGE 204

$T S-A Z|O| \varepsilon W T-|A|$

Tag:

P\&ID: $\begin{aligned} t+2-131069 & \text { SH -1 } \\ -2-132078 & -5 H-1\end{aligned}$

LOOP:

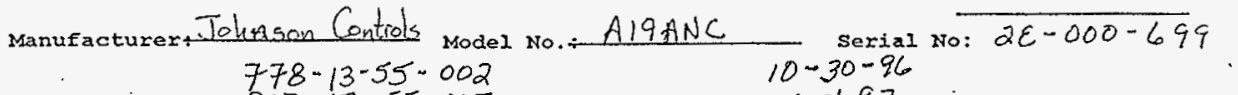

MTE Instr. No:- $\frac{817-13-55-015}{817 \cdot 45-08-040} \quad$ Due Date: $\frac{5-21 \cdot 97}{2-5-97}$

Input Range $\frac{\mathrm{Pg}_{1}^{\mathrm{C}} \mathrm{3}^{\mathrm{Ck}}}{\mathrm{C}}: 0-150$

Tolerance $2 \%$

Units: ${ }^{\circ} \mathrm{F}$

Input output

$40^{\circ} \mathrm{F}$ SiWTTCN

Lo: Hi: As Found In/Out As Left

$37 \quad 43 \quad \Delta / A-N / A$

$4+\circ 39)^{\circ} \mathrm{F}$

(signoff)Calibrated By:

Date: $-6 \cdot 3 \cdot 96$

Location: 101AZ COOLNE WBTER TOWER

Comments Low Time. SwITCH. Swatch closed on 40\% to to energize beaters.

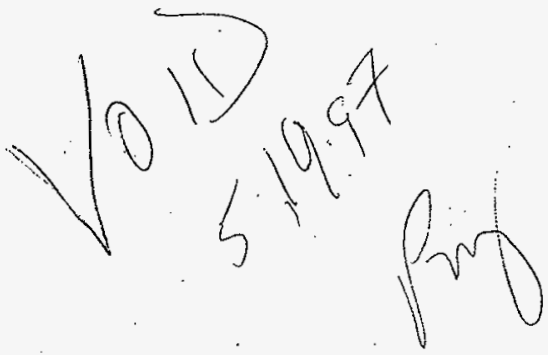


Tag: TS-AZ101EWT-1A1

P\&ID : H-2-131069, SH 1

LOOP :

Manufacturer: Johnson Controls mode1 No.: AIGANC Serial No: $2 E-000 \cdot 699$

$817-13.55 \cdot 015$

MTE Instr. No: 817.45 .08 .040 $817-13 \cdot 35 \cdot 025$
5.21 .97

Date: $\frac{2 \cdot 28 \cdot 99}{7-24 \cdot 97}$
Input Range 0-150

Units: DEG $F$

Input Output

$40 \quad$ switch
Tolerance $2 \%$

Lo: Hi: 43

As Found

In/Out

As Left

37 36 DUT 40

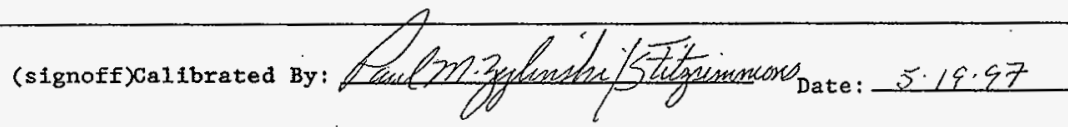

Location: 1O/AE COOWNG WATER TOWER.

Comments LOW TEMP. SWITA, SWTEN CLOSES ON $40^{\circ} \mathrm{F}$ to ENZRGHE WEATERS CALIBRATE PER VENDOR DATA SET TO TURN ON AT 40 DEG F 
Tag: LAIL_AZ1EWT_1

CntIr: 52

Remarks:

Location:

Actuation Method: TURNEP tEAK DETECTORQP ANP

sensor calibration: $N / A$

$\square$ pex procedure (data attached)

(signoff)

Setpoint:

Loop Tolerance:

Process State

Lo (0): IOW

Hi (1): NORMAL

\begin{tabular}{|c|c|c|c|}
\hline & IOI & Screen1 & Screen2 \\
\hline Color & ICU3-15 & 08EvpAZ1 & \\
\hline
\end{tabular}

3

2

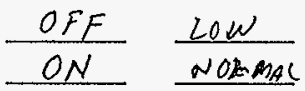

Annunciator(s): $\quad$ Priority

Hi: 0

Lo: 2
Alarm color (actual)

Yeccow

other:

MIE Instr, No:

Due Date:

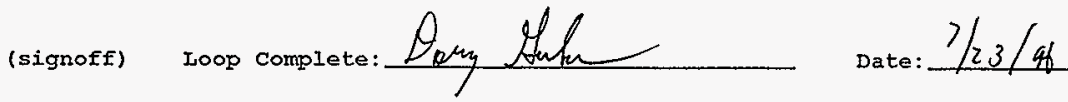


Tag: FI_AZ2_EWR_1 I/O Type: AI

Cntlr: 51 Chan No: 24
Description: EwrHdx Flow

P\&ID: $\mathrm{H}-2-131070$
Logic:

Remarks :

Location:

Actuation Method: $\frac{\text { calibated 4-20 Source on ovtpot side of }}{\text { Transmitter }}$

Sensor Calibration:

$5 \mathrm{pt} \mathrm{cal}$ per procedure $\square$ Data

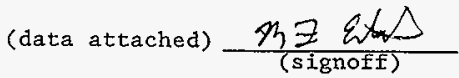

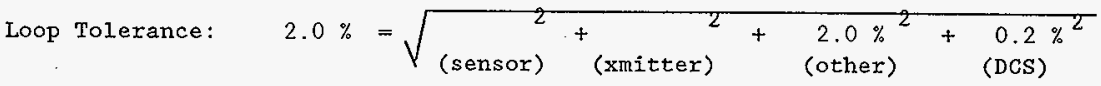

Units: GPM

Process Input

Lo: $\quad 0.00$

Hi: $\quad 300.00$

Annunciator(s):

Max:

HiHi :

$\mathrm{Hi}$ :

Lo:

LoLo:

Min:
Readout Tolerance

$$
-6.03-6.03
$$

$293.97-306.03$

Priority Setpoint 99999.99 99999.99 99999.99 170.00 $-9999.99$ $-9999.99$

0
IOI LCU3-22

Screen1 09EvpAZ2

$\frac{0.02}{299.81}$

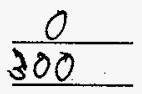

Screen2 gray 72 0.05 299.54
Tolerance

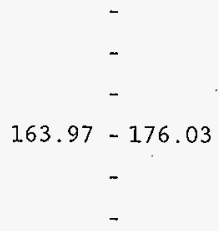

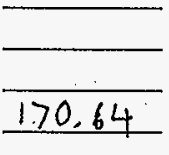$$
-9999.99
$$

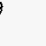

\section{other:}

\section{MTE Instr. No: $81>-13-55-023$ Due Date: $3-26-97$}

(signoff) Loop Gomplete: $\overline{2} \exists$ Elat

Date: $6-13-96$ 
$5 / 1 / 97$

Tag: FT-AZ102EWR-1
W-030 CALIBRATION DATA SHEET
HNF-SD-W030-TD-003,

REV. 0, PAGE 208

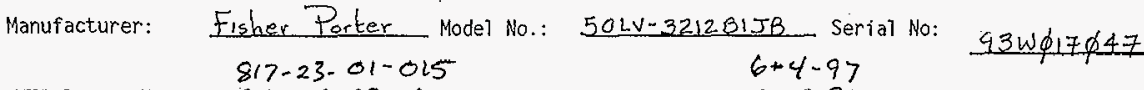

MTE Instr. No: $817 \cdot 45 \cdot 08 \cdot 040$

Due Date: $\quad 2 \cdot 28.98$

Input Range $0-300(0-64 \mathrm{~Hz})$

Tolerance $2 \%$

Units: gem

\begin{tabular}{ccccccccc} 
Input (Hz) & Output(ma) & $(\%$ meter $)$ & Lo: & Hi: & As Found & In/Out & As Left \\
0 & 4.00 & 0 & $3.68(-2)$ & $4.32(2)$ & 3.97 & $(0.5)$ & IN & $3.97(0.5)$ \\
8 & 6.12 & 13 & $5.80(11)$ & $1.44(15)$ & 6.09 & $(13.5)$ & IN & $6.09(13.5)$ \\
16 & 8.24 & 26.5 & $7.92(24.5)$ & $8.56(28.5)$ & 8.22 & $(26.5)$ & IN & $8.22(26.5)$ \\
32 & 12.49 & 53 & $12.17(51)$ & $12.81(55)$ & 12.48 & $(53)$ & IN & $12.48(53)$ \\
64 & 20.98 & - & 20.66 & 21.30 & 20.98 & $(\mathrm{~N} / \mathrm{A})$ & IN & $20.98(\mathrm{~N} / \mathrm{A})$ \\
\hline
\end{tabular}

(signoff) Calibrated By:

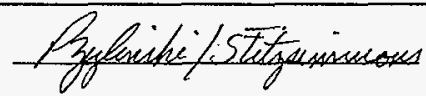

Date: $5 \cdot 16.97$

Location: $102 \mathrm{AZ}$ Cooling Water tower

Comments Calibration factor $(\bar{k})=24.115 \mathrm{C} / \mathrm{gal} \odot 93^{\circ} \mathrm{F}$

$f_{0}=120.6 \mathrm{~Hz}$.

Span factor $=1324$

$f_{\text {test }}=60.3 \mathrm{~Hz}(\max )$

Verified zeno: Spare at macon.

Pry

$$
\begin{gathered}
0=-1 \mathrm{gpm} \\
53 \%(159 \mathrm{gpm})=160 \mathrm{gpm}
\end{gathered}
$$


Tag: FT-AZ102EWR-1

P\&ID: H-2-131070, SH 1

I,OOP: H-2-131334, SH 11

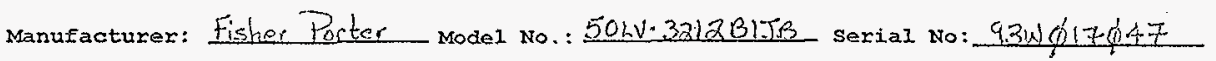
MTE Instx. No: $817 \cdot 45-08-040$ Due Date: $\begin{array}{r}2-5 \cdot 97 \\ \frac{6-9.97}{6}\end{array}$

Input Range $0-300$ Tolerance $2 \%$

Units: $g p^{m}$

Input (Hz) output (ma) $\quad\left(\begin{array}{c}\% \\ \text { meter) Io: }\end{array}\right.$

日i: As Found In/out As Left

8 4.00 6.12

16 Pmy $8.49 \times 8.24(26.5) 792(24.5856(28.5)$

$32 \quad 12.49$

$64 \quad 20.98$

(53) $12.17(51) 12.81(5.5)$

$-(+06) 20.66(-) 21.30$

b.12. 6

(signofe)calibrated By: focel mighensti.

Date: $6 \cdot 12 \cdot 96$

Iocation: $A Z$ CoOLWE WATER TOWEr

comments Calibration factor $(\vec{k})=24.115 \mathrm{C} / \mathrm{gal} @ 93^{\circ} \mathrm{F}$ $f_{0}=120.6 \mathrm{~Hz}$

Span factor $=1324$

$f_{\text {test }}=60.3 \mathrm{~Hz}$ (max)

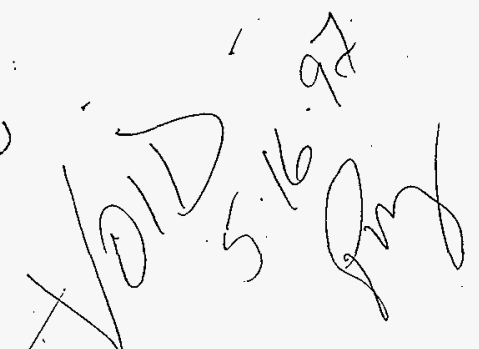


Tag: PDAH_AZ2WE_2 I/O TYPe: DI Descxiption: AZ2EWFtrDpHi

CntIr: 53 Chan No: 20 P\&ID: H-2-131070 Iogic:

Remarks:

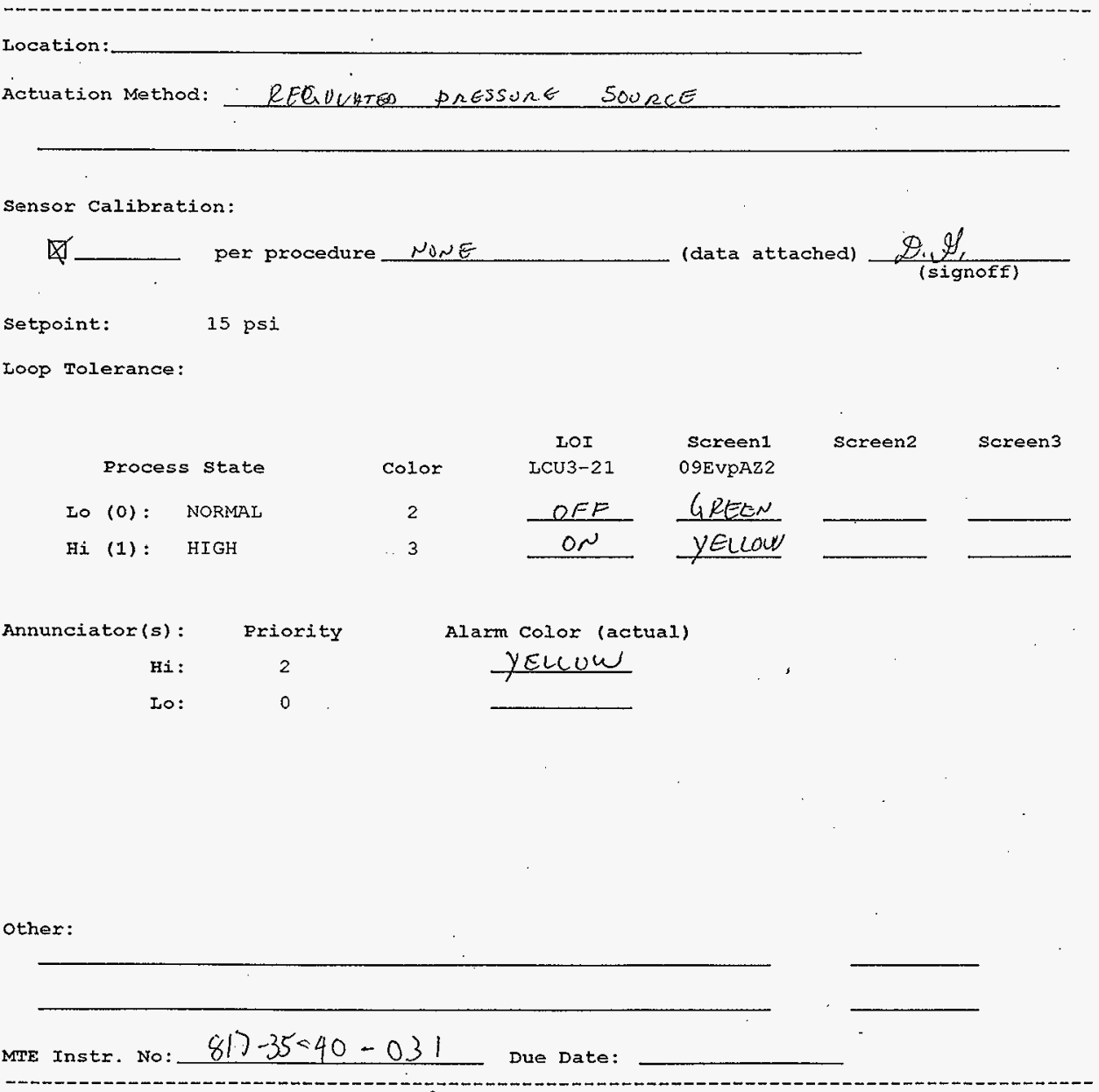

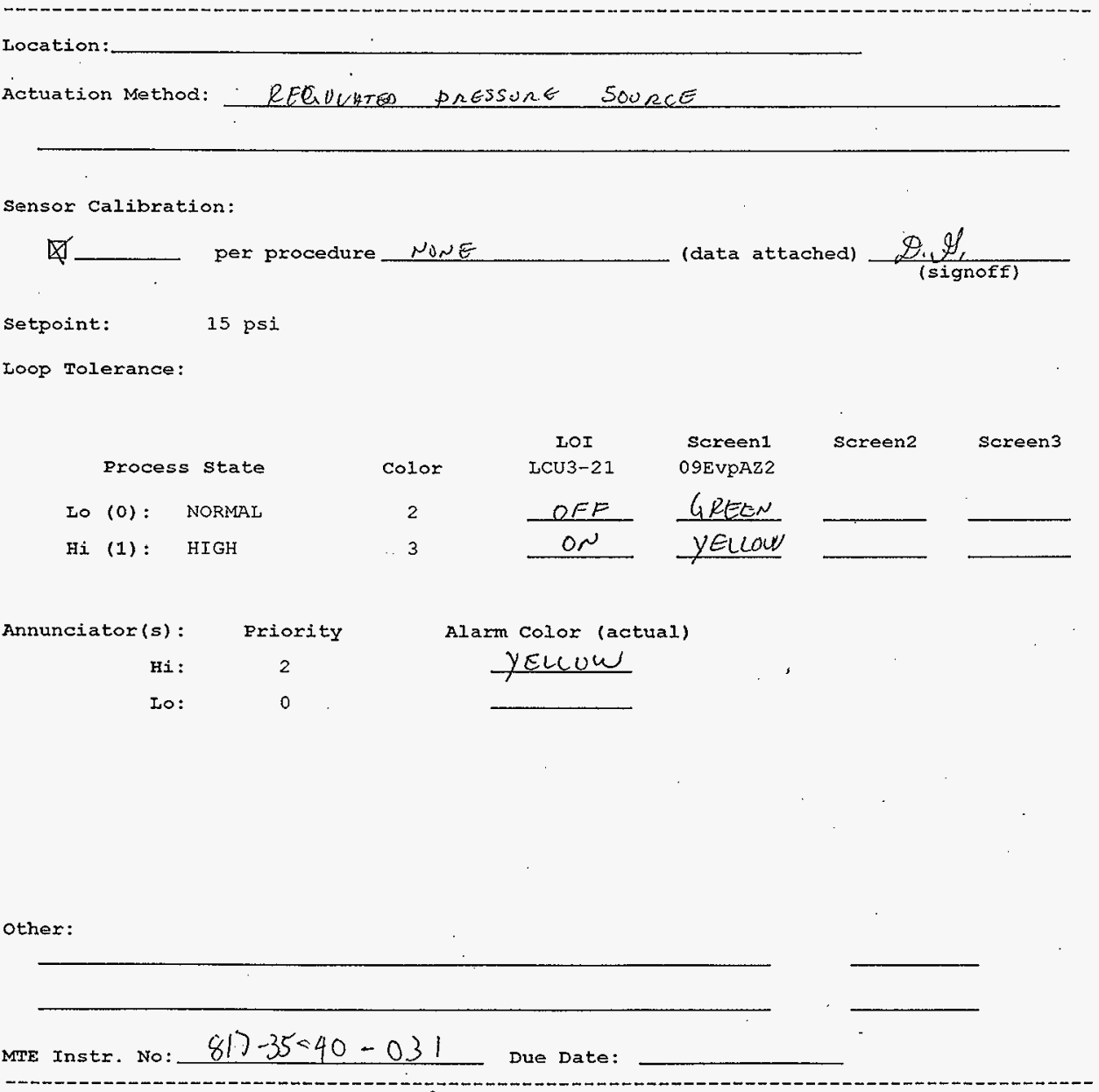

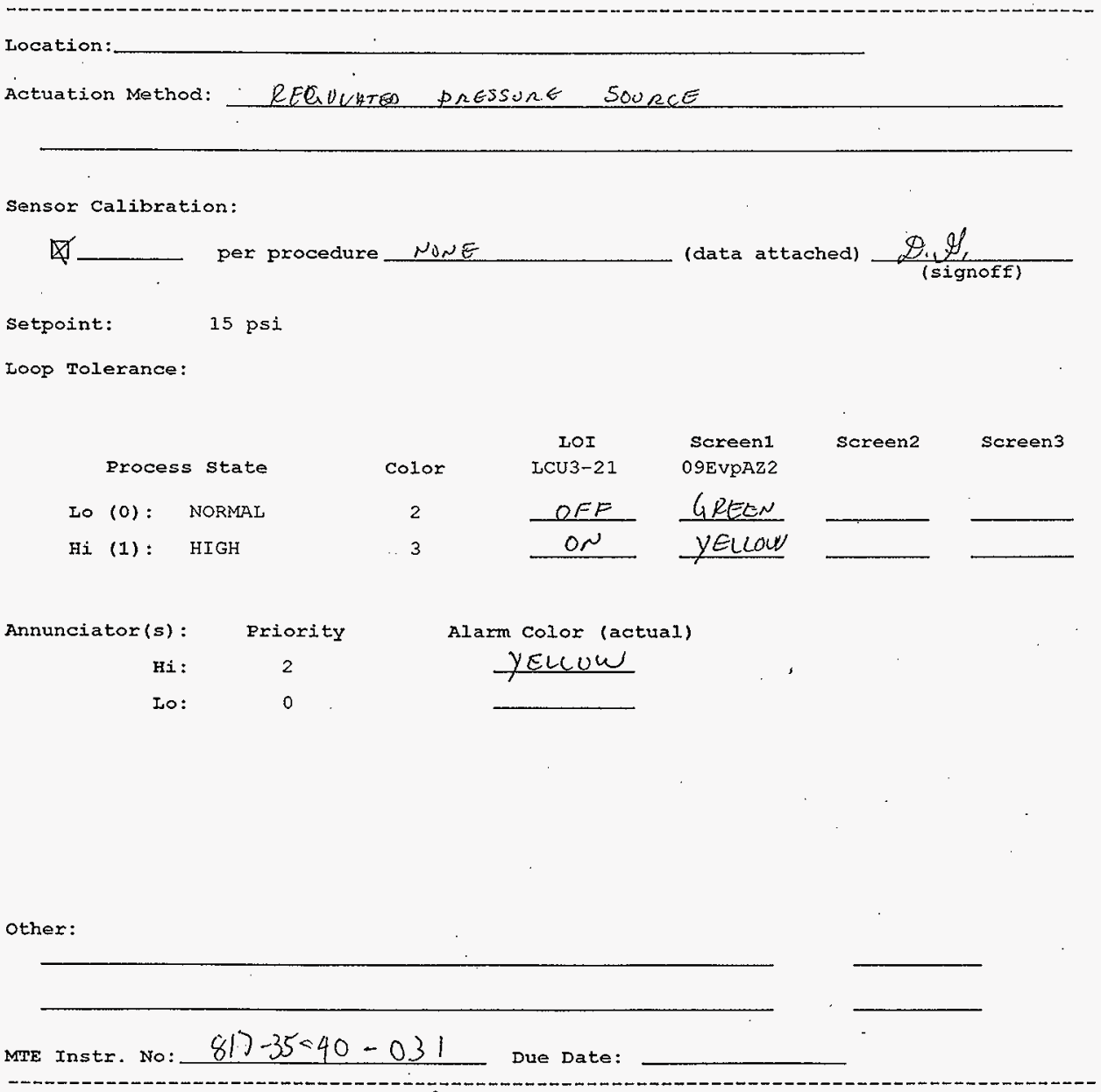

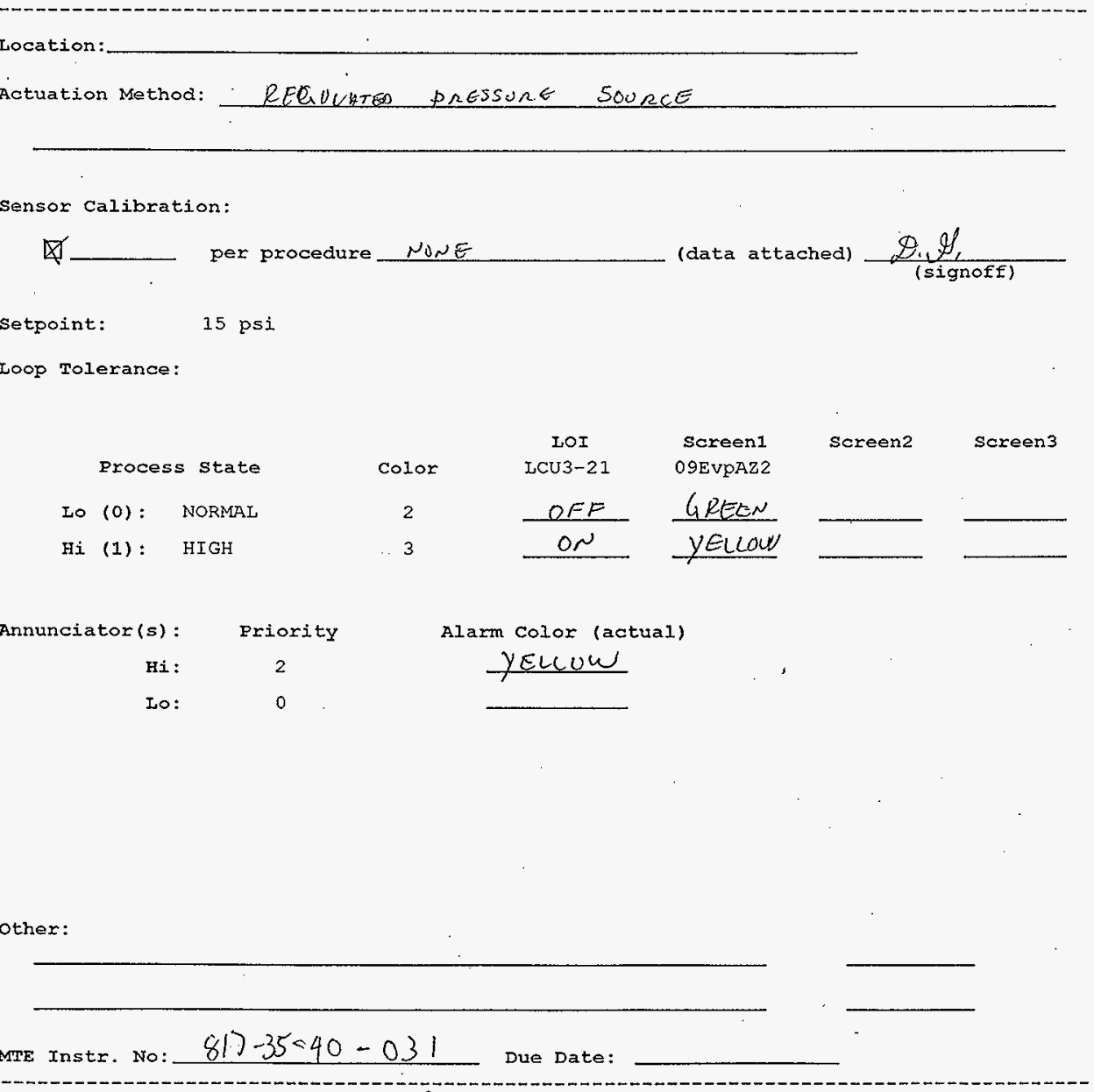

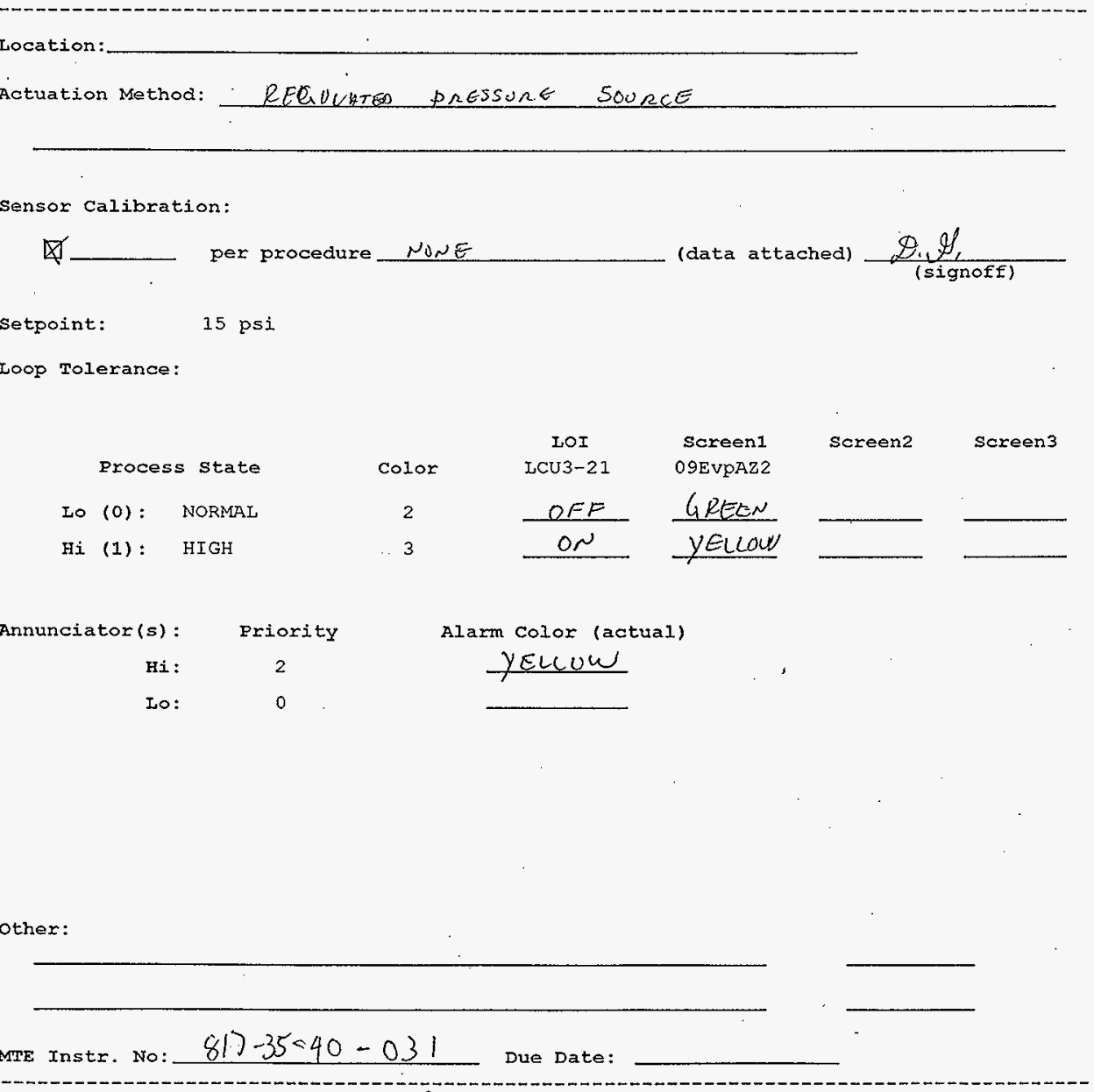

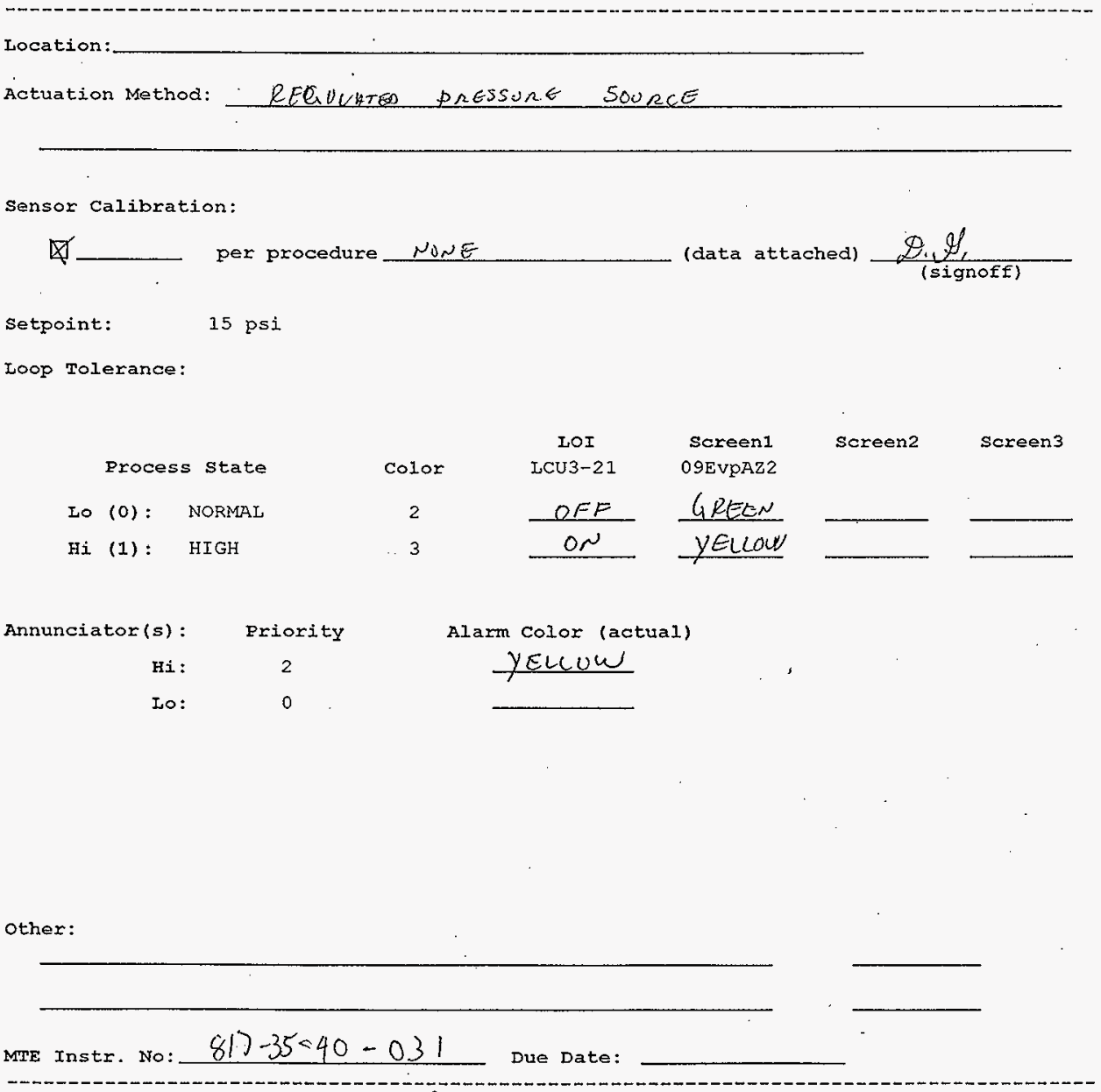

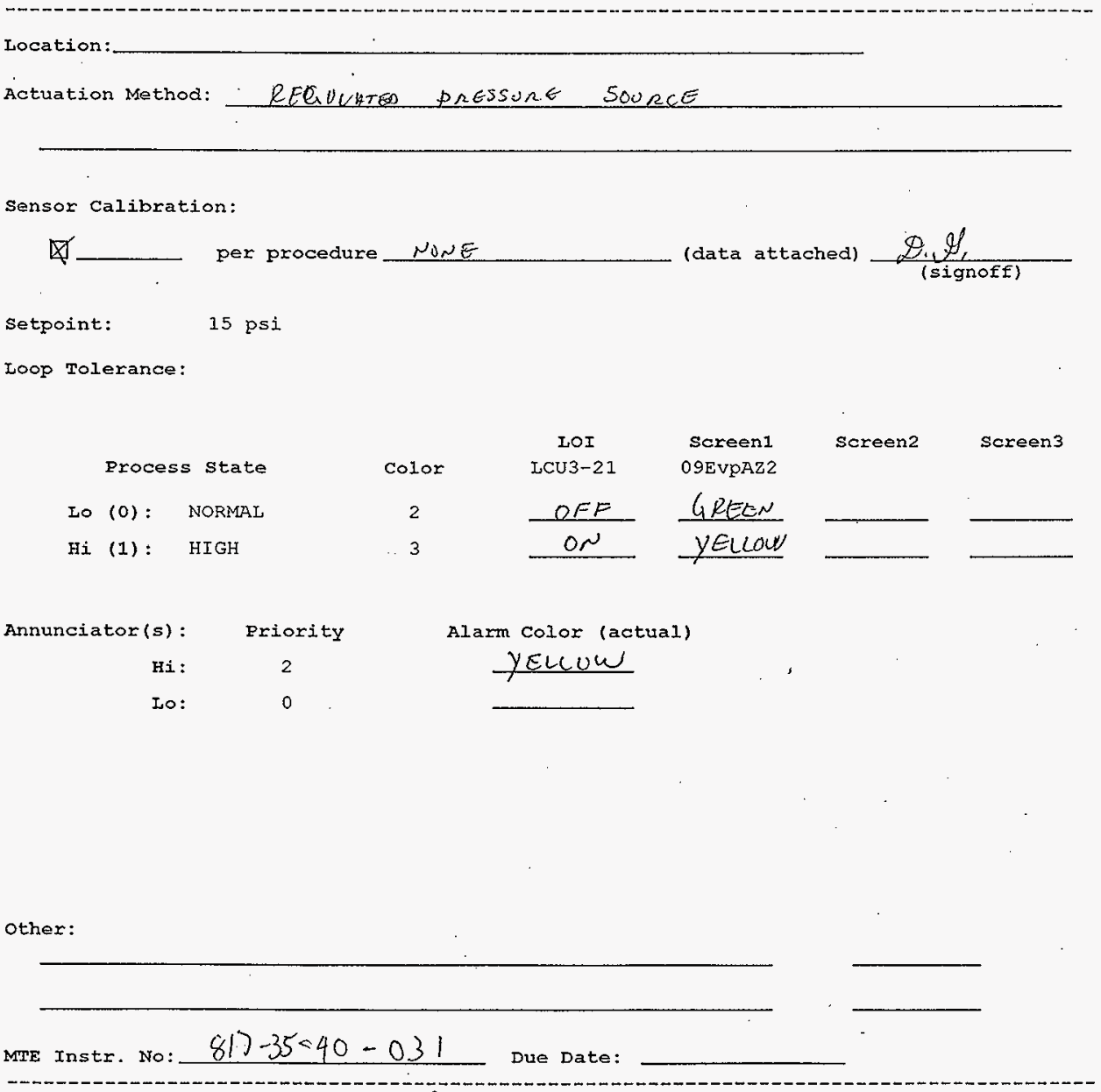

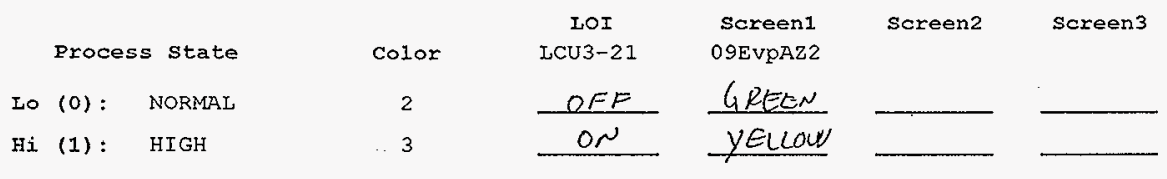

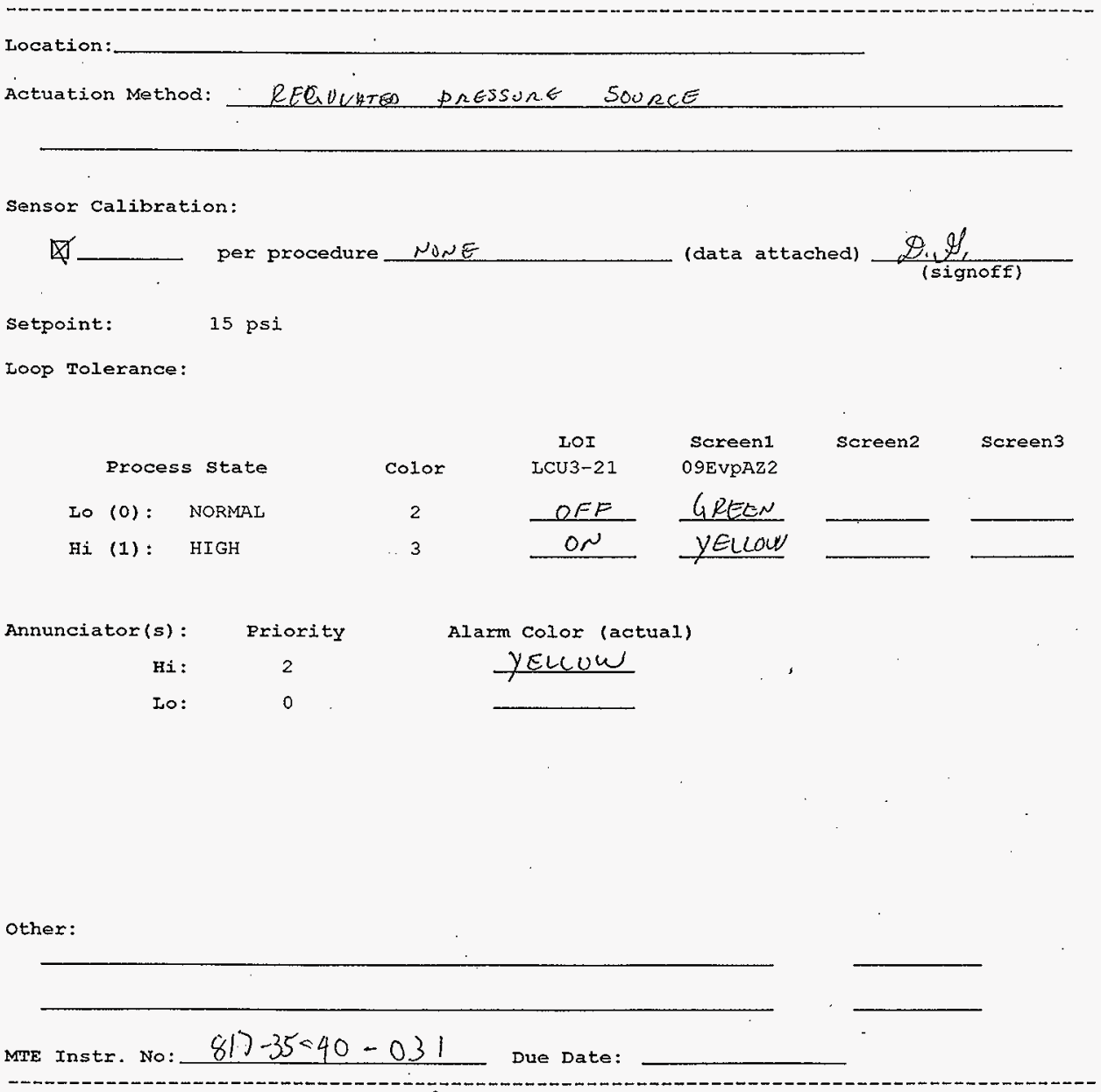

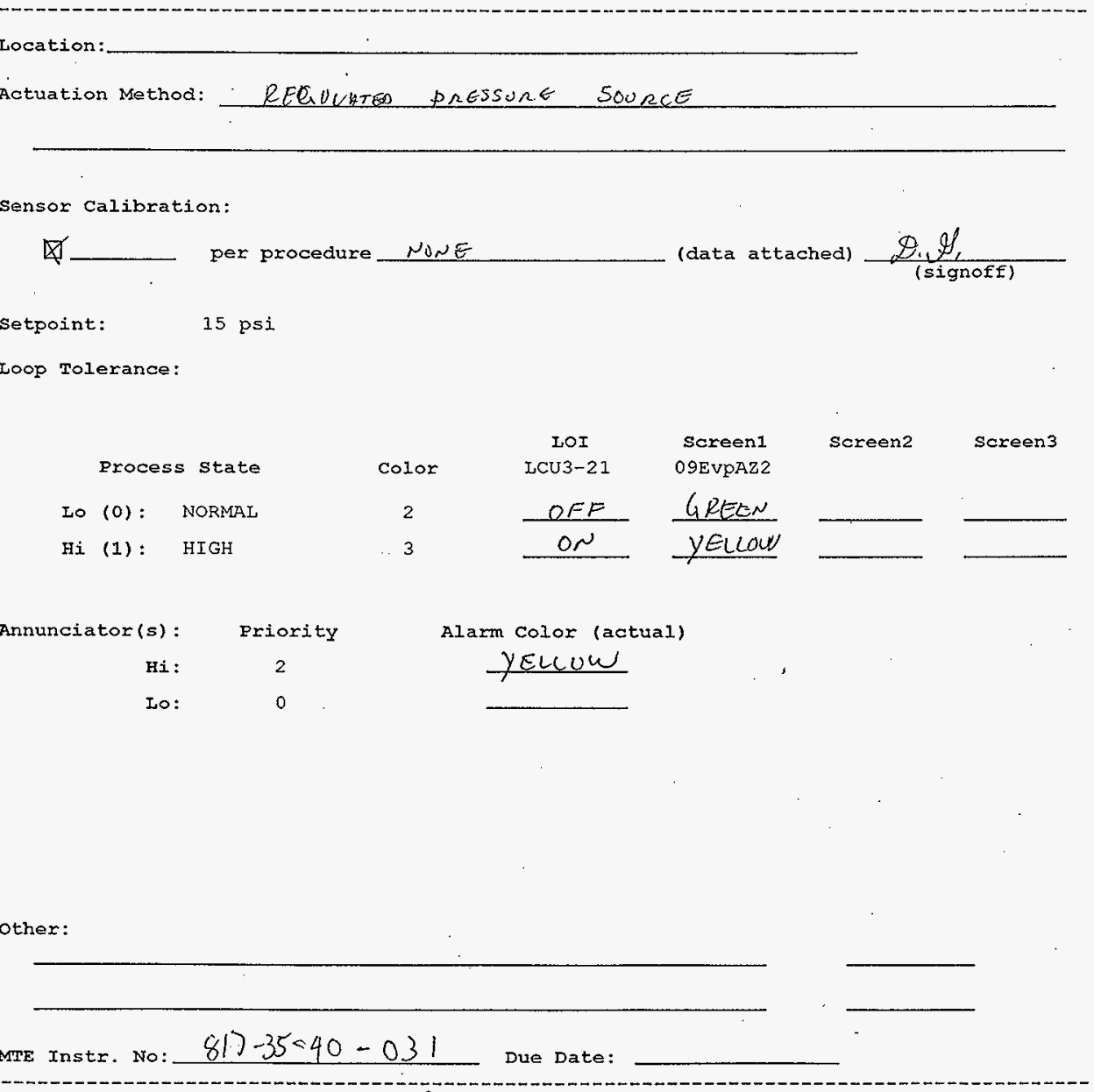

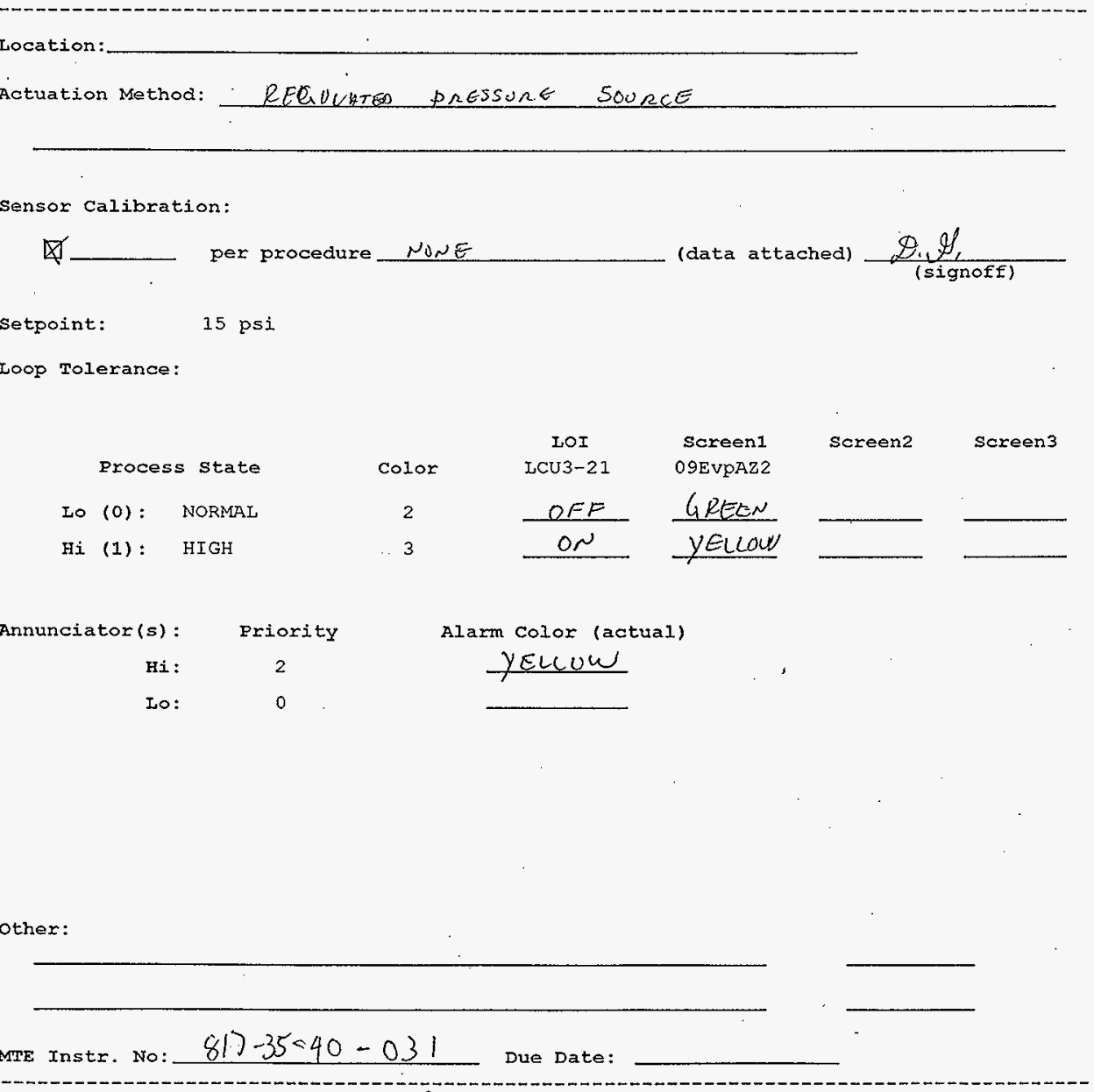

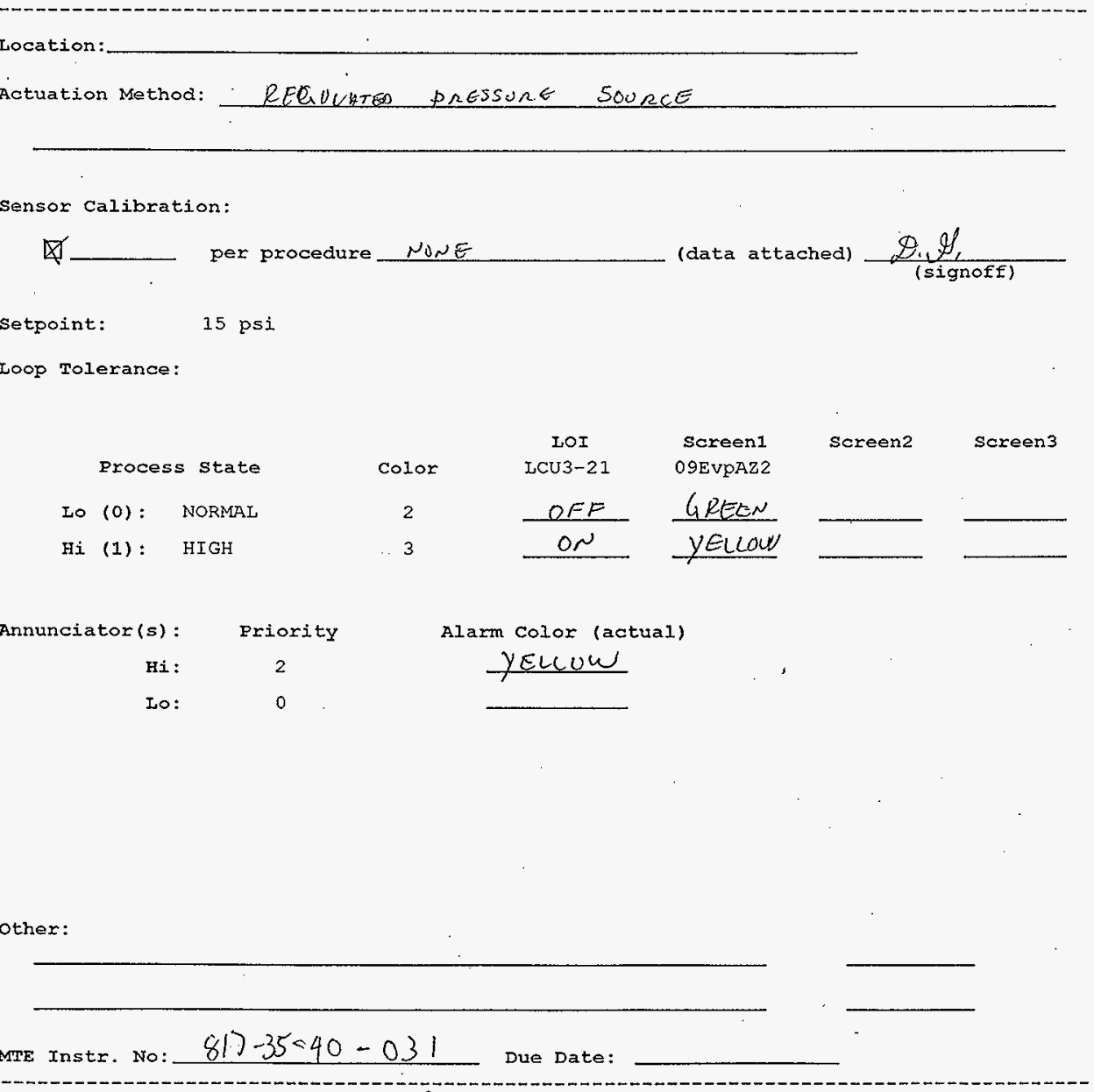

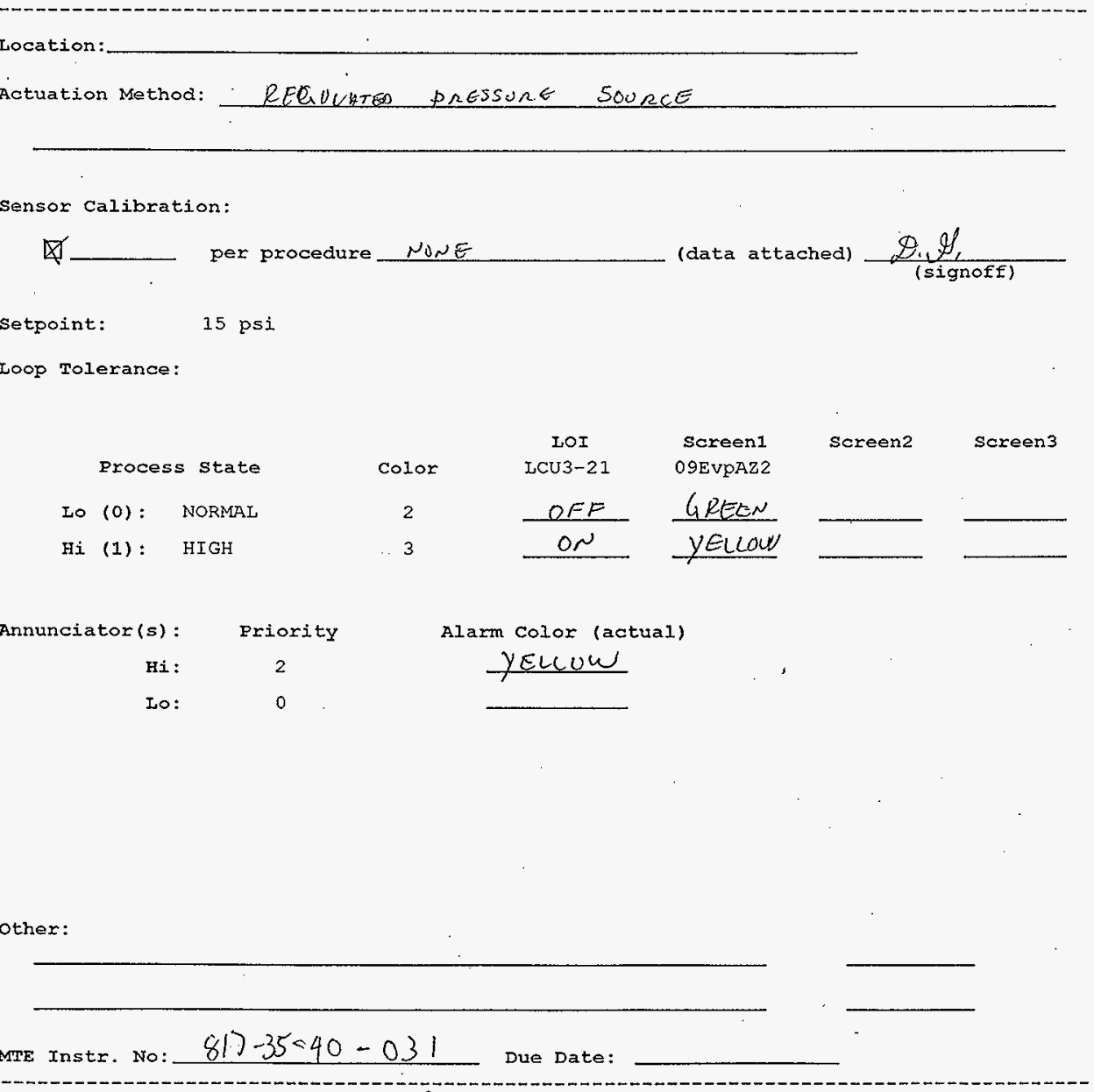

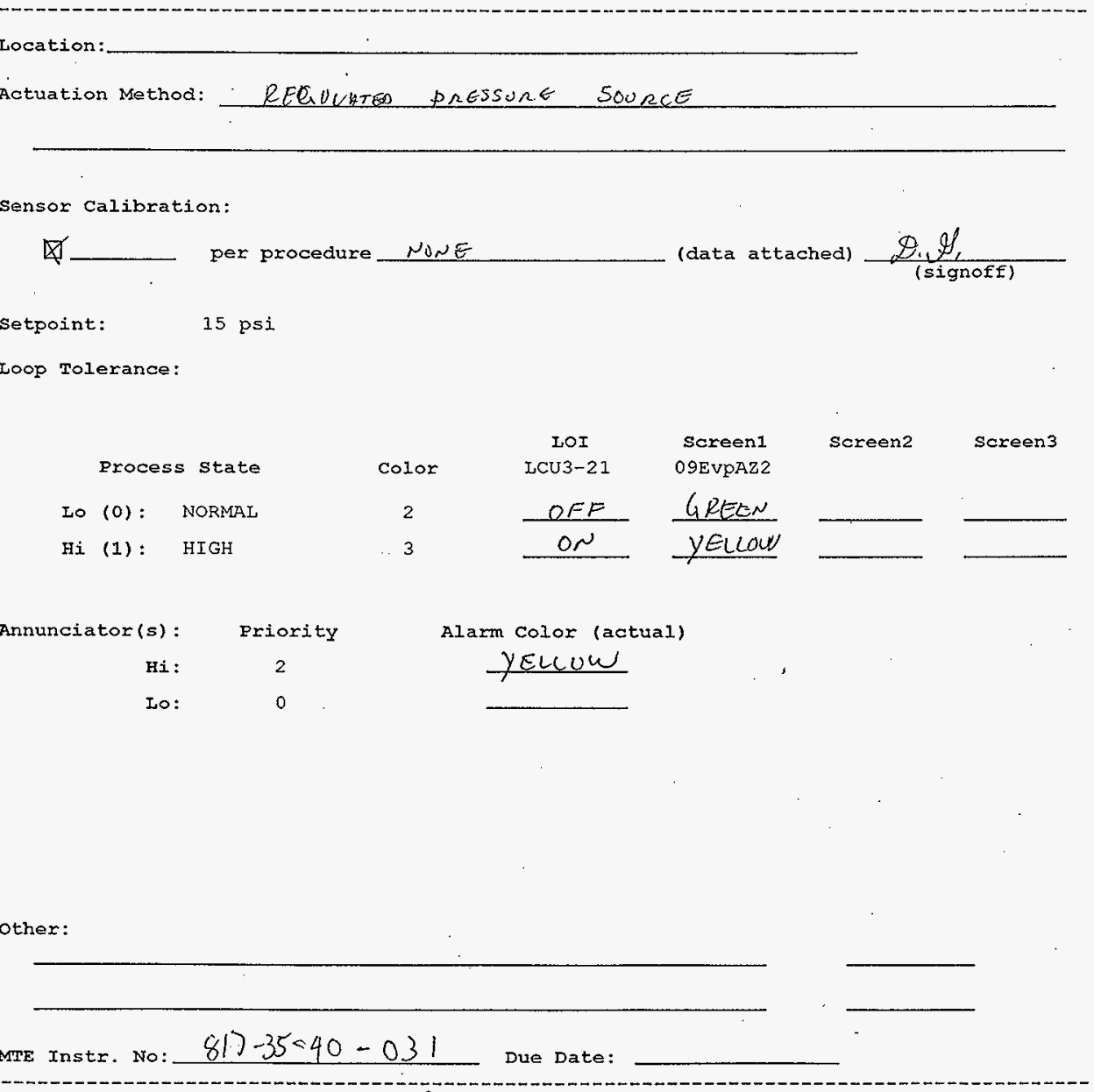

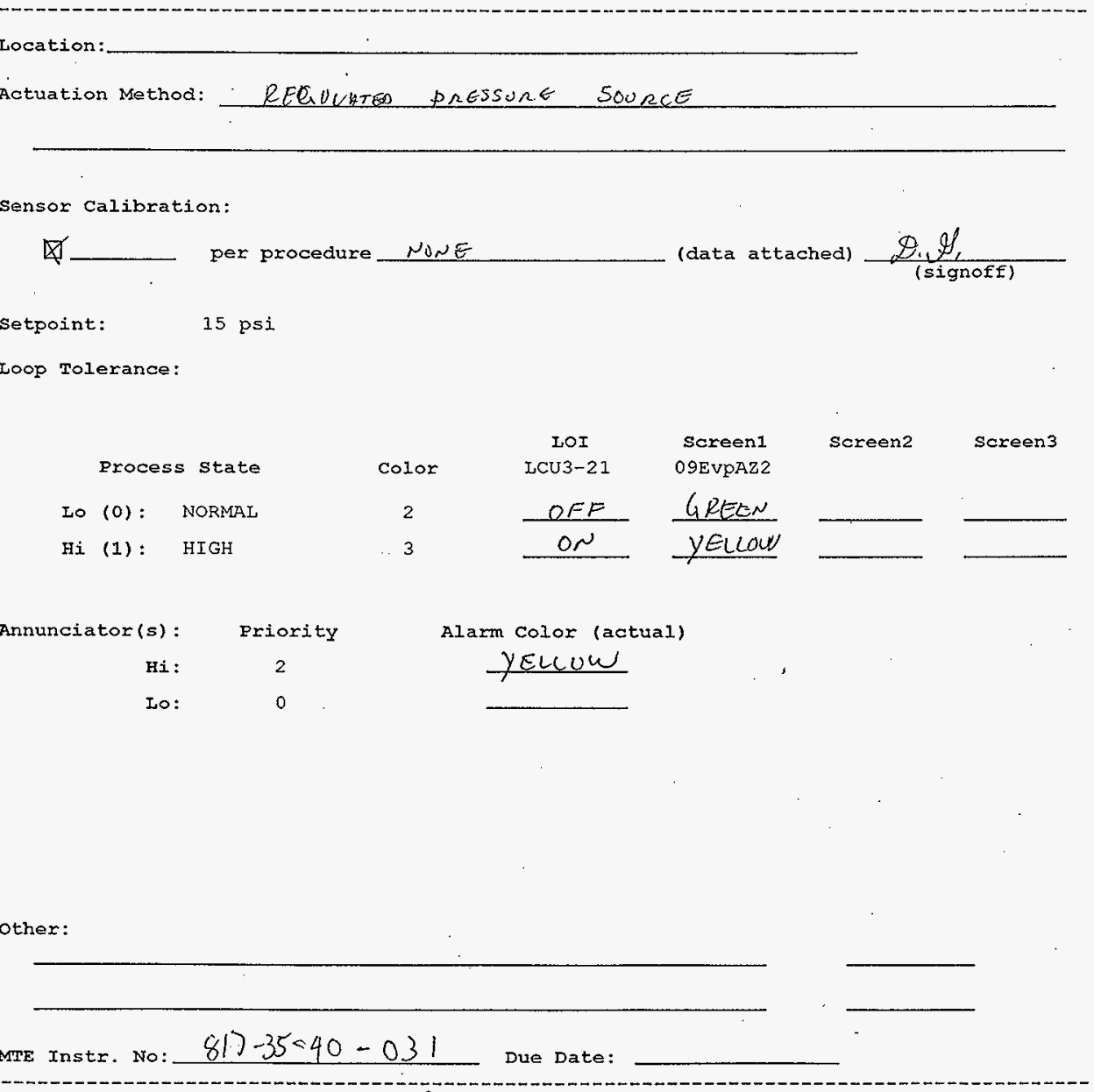

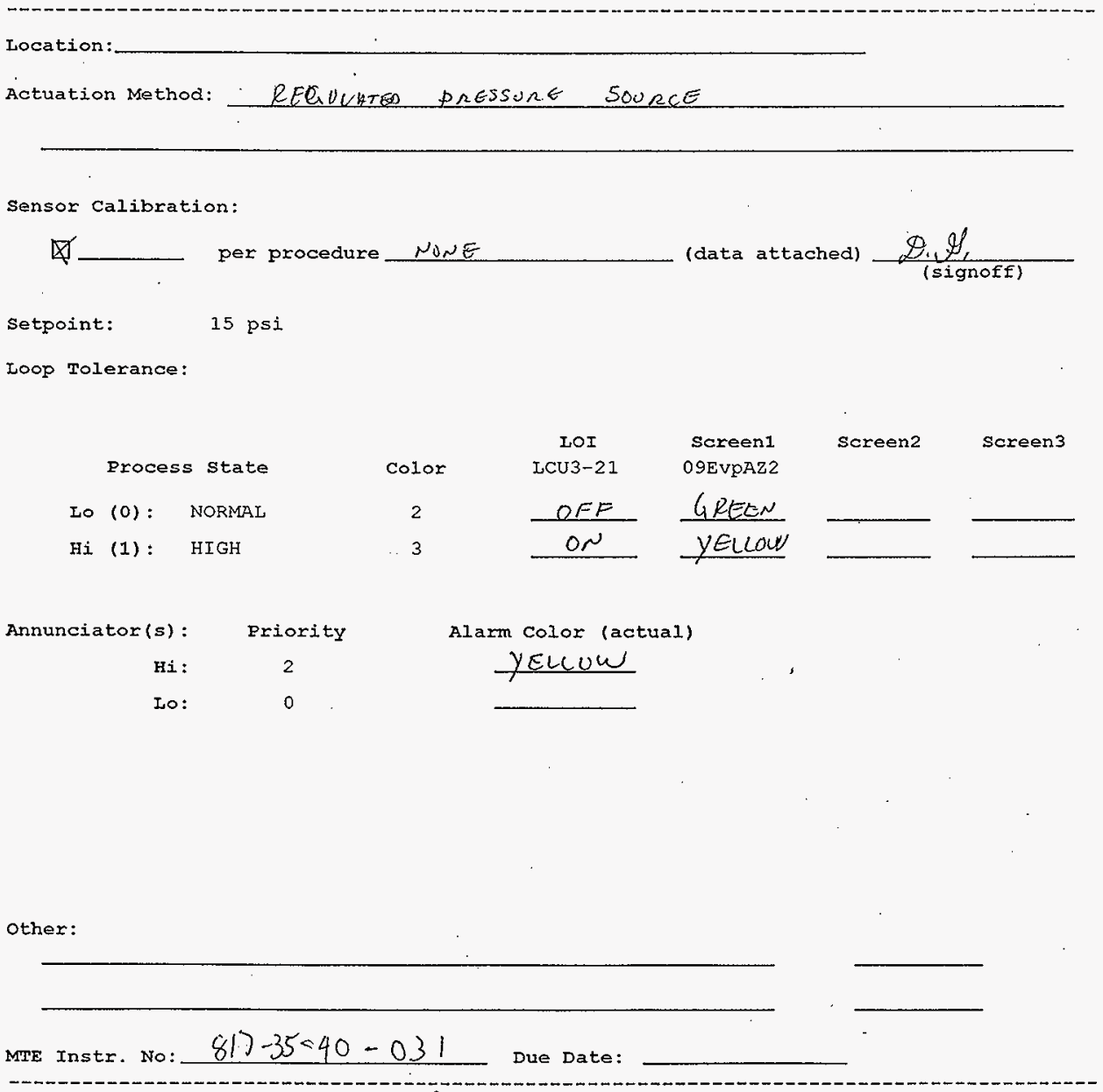

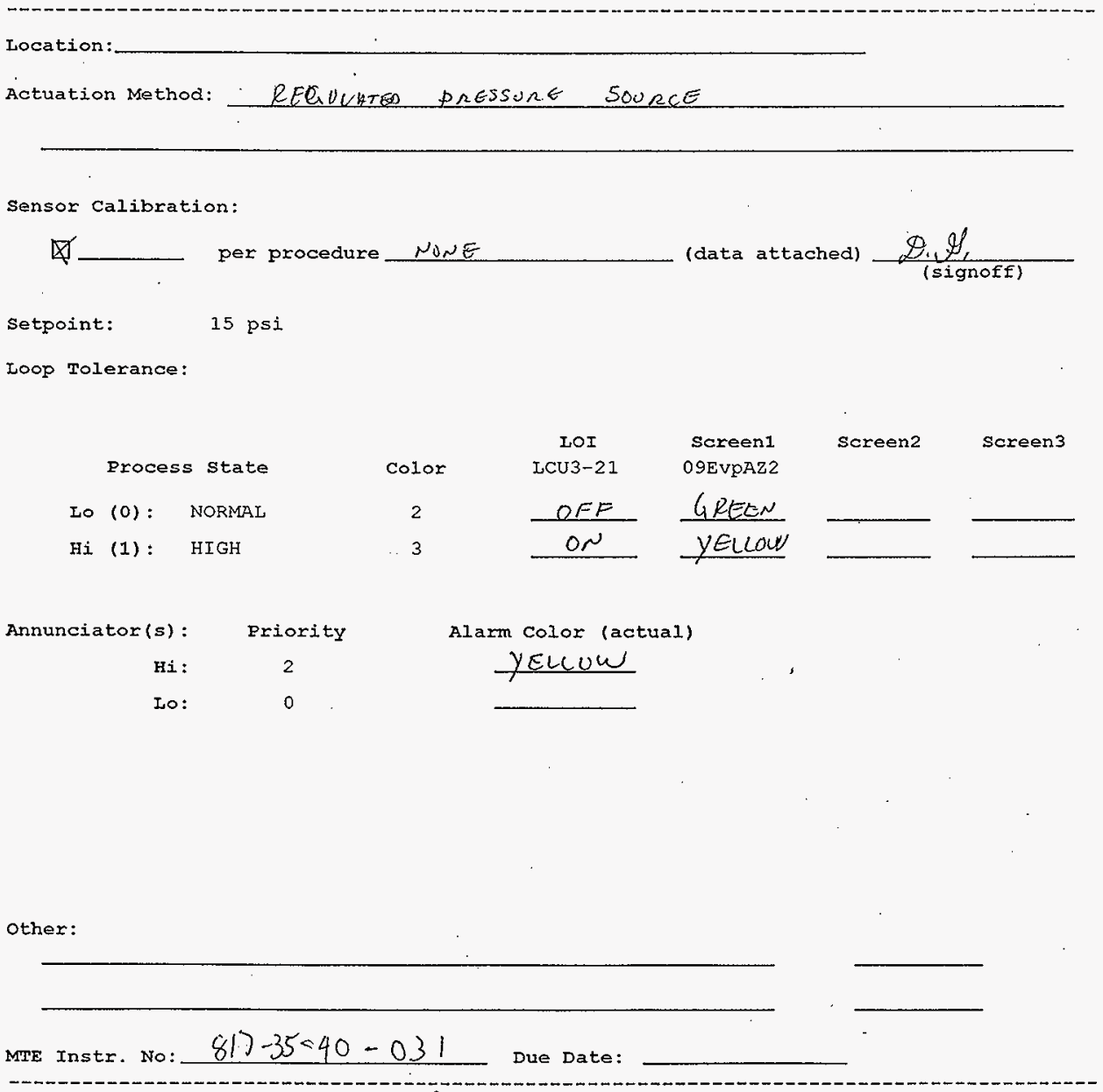

(signoff) Loop complete:

Date: $9 / 16 / 96$ 
Tag: PDIS-A2102WF-2

Manufacturer: DWYER

Mode1 No. : $43220 \mathrm{C}$

Serial No: 2E-000-770

MTE Instr. No: $8 / 7-35-40-03 /$ Due Date: $7-3 /-97$

Input Range $0-20$

Units : PSI

Input output

0.00

$5 \quad 5.00$

$10 \quad 10.00$

$15 \quad 15.00$

$20 \quad 20.00$

\section{Tolerance $2.00 \%$}

$$
+/-0.40
$$

Lo: Hi: As Found In/Out As Left

\begin{tabular}{lllll}
-0.40 & 0.40 & 0.0 & $I_{n}$ & 0.0 \\
\hline 4.60 & 5.40 & 4.9 & $I_{n}$ & 4.9 \\
\hline 9.60 & 10.40 & 10.0 & $\operatorname{In}$ & 10.0 \\
\hline 14.60 & 15.40 & 15.0 & $I_{n}$ & 15.0 \\
19.60 & 20.40 & 19.8 & $\operatorname{In}$ & 19.8 \\
\hline
\end{tabular}

(signoff)Calibrated By: R. Wilmoth Q. Anzos Date: $7-18-97$

Location: AZIO2 CONDENSER

Comments

SET SWITCH TO ALARM AT 15PSI, FUNCTIONAL TEST: DOES ALARM COME IN AT MICON? YES NO $\swarrow$

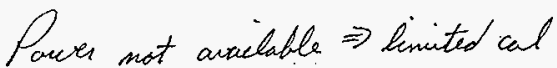


Manufacturer:

Model No.:

Serial No:

Mre Instr. No: $817-35-40 \cdot 031$

Due Date:

Input Range $0-20$

Units: PSI

Input output
Tolexance $5 \%$

\begin{tabular}{|c|c|c|c|c|}
\hline IO: & $\begin{array}{l}\text { Hi: } \\
15 \text { QSF }\end{array}$ & $\begin{array}{l}\text { As Found } \\
N e w\end{array}$ & $\begin{array}{l}\text { In/Out } \\
I N\end{array}$ & $\begin{array}{l}\text { As Left } \\
150 s y\end{array}$ \\
\hline
\end{tabular}

(signoff)calibrated By: $\frac{k=3}{3}$ / Pat Hurson Date: $4-16-96$

Iocation:

Comments

SET SWITCH TO ALARM AT 15PSI D

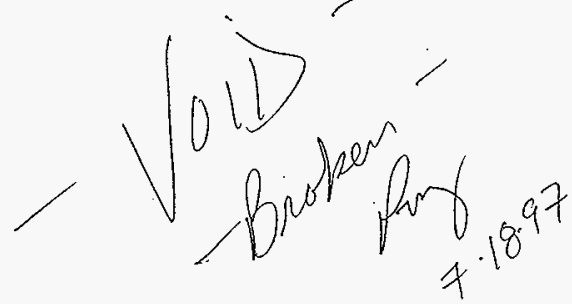


Tag: II_AZ2_EWP_1A I/O Type: AI

Description: EwPumplaCurrent

Cnt1x: 51

Chan No: 25

P\&ID: $H-2-131070$

Logic: Y412

Remarks :

Location:

Actuation Method: pun motor t compure cribrited anpeter to DCS

sensor calibration: N/A - Tronsmitter Factery calibrated

口 5 pt cal

$\square$ per procedure (data attached)

Loop Tolerance:

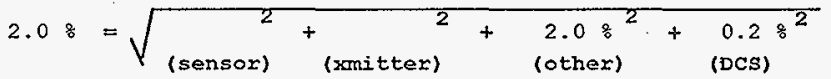

Units: AMP

Process Input

Readout Tolerance

Lo:

0.00

$-.40 /$

.40

Hi :

$$
\text { Running current } 1000 \mathrm{mzc}
$$

9.4

Annunciator(s):

$$
\text { Priority }
$$

Setpoint

99999.99

Max:

$$
0
$$

BiHi:

Hi:

$$
0
$$

99999.99

Lo:

$$
0
$$

99999.99

LOLO:

Min:

\begin{tabular}{|c|c|c|}
\hline $\begin{array}{c}\text { IOI } \\
\text { LCU3-22 }\end{array}$ & $\begin{array}{c}\text { Screen1 } \\
\text { 09EvpAZ̨2 }\end{array}$ & $\begin{array}{l}\text { screen2 } \\
\text { of main } \\
\text { of Totglim }\end{array}$ \\
\hline 0 & 0 & Empty \\
\hline 9.46 & $3+9.52$ & filled \\
\hline
\end{tabular}

0

$-9999.99$

$-9999.99$

$-9999.99$
Screen 3

Tolexance

Trip Point

$$
\begin{aligned}
& 1 \\
& 1 \\
& 1
\end{aligned}
$$$$
\text { / }
$$$$
\text { / }
$$

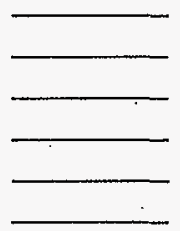

othex:

Two Point check. Compared Ammete, reading to Des reading

MIE Instr. No: $988-45-02-006$ Due Date: 1/24/97

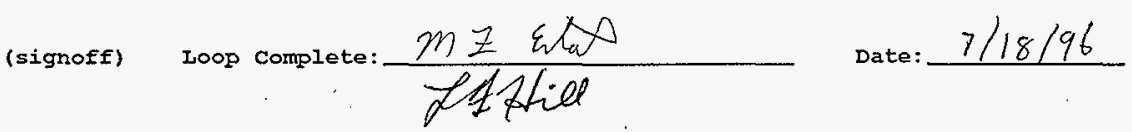


$5 / 14 / 97$

W-030 CALIBRATION DATA SHEET

HNF-SD-W030-TD-003,

REV. 0, PAGE 214

Tag: .IT - AZ102EWP-IAji:

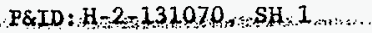

LOOP: H-2-13̧1334, SH 6

Manufacturer: KATY NSTRS. Model No.: 420 Serial No:

$817 \cdot 45 \cdot 08 \cdot 040$

$2 \cdot 28 \cdot 98$

MTE Instr. No: $817 \cdot 45: 02 \cdot 004$

Due Date: $5 \cdot 16.97$

Input Range $D-20$

Tolerance $2 \%$ - output

Units: $A$

$$
\begin{array}{ccccccccc}
\text { Input (A) } & \text { Output }(\mathrm{ma})(\mathrm{MicoN}) & \text { Lo: } & \text { Hi: } & \text { As Found } & \text { In/Out } & \text { As Left } \\
0 & 4.00 & 0 & 3.68(-1) & (1) & 4.00(0) & \text { in } & 4.00 & (0) \\
20 & 20.00 & 20 & 19.68(19) & 20.32(21) & 20.00(20) & \text { in } & 20.00 & (20)
\end{array}
$$

(signoff)Galibrated By:

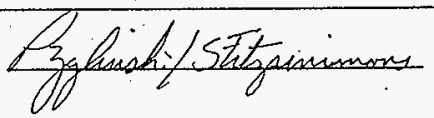

Date: $5 \cdot 14 \cdot 97$

Location: $102 \mathrm{AZ}$ COOING WATER TOWER (PUMP IA)

comments Functional. Factory calibrated; check zero and span.

Use current generator w/ calibrated MTE clamp on meter 
Tag: II_AZ2_EWP_1B IB I/O TYPe: AI

Description: EwPump1BCurrent

Cnt1r: 51 Chan No: 26 .

P\&ID: $H-2-131070$

Logic: $Y 414$

Remarks :

Location:

Actuation Method: Run mitor \& compare ammeter te $D(S$

sensor calibration: N/A Transmitt Factory calrodid. $\square 5$ ptcal per procedure

(data attached)

(signoff)

Loop Tolerance: $\quad 2.08=\sqrt{\text { (sensor) }^{2}+\text { (xmitter) }^{2}+\text { (other) }^{2.0 \%}{ }^{2}+0.28^{2}}$

Units: AMP

Process Input

Readout Tolerance

Lo: $\quad 0.00$

$-.40 / \quad .40$

Hi: $\quad 20.00$

$19.60 / 20.40$

9.2

Annunciator(s): Priority setpoint

Tolerance

Trip Point

$\begin{array}{ccc}\text { Max: } & 0 & 99999.99 \\ \text { HiHi: } & 0 & 99999.99 \\ \text { Hi: } & 0 & 99999.99 \\ \text { LO: } & 0 & -9999.99 \\ \text { LoLO: } & 0 & -9999.99 \\ \text { Min: } & 0 & -9999.99\end{array}$

other: Two point check. compared. Ammeter reading to OCS reading.

\begin{tabular}{|c|c|c|c|}
\hline $\begin{array}{c}\text { LOI } \\
\text { LCU3-23 } \\
0\end{array}$ & $\begin{array}{c}\text { Screen1 } \\
\text { 09Evpaz2 } \\
0\end{array}$ & $\begin{array}{l}\text { screen2 } \\
\text { odmain } \\
04 \text { pofilay } \\
\text { Empty }\end{array}$ & Screen 3 \\
\hline 9.36 & 9.39 & F.lled & . \\
\hline
\end{tabular}

Min:

1
1
1
1
1

MIE Instr. No: $\quad 988-45-02-006$ Due Date: $1 / 24 / 47$

(signoff) Loop complete: $\frac{m \geq \operatorname{lin} D}{2 \neq 26 \text { ill }}$

Date: $7 / 18 / 96$ 
Manufacturer: KATY NSTRS. Model No.: 420 ' Serial No:

\section{$817 \cdot 45 \cdot 08 \cdot 040$}

MTE Instr. No: $8 / 7 \cdot 45 \cdot 02 \cdot 004$
$2 \cdot 28 \cdot 98$

Due Date: $5 \times 16.97$

$5 \%$-screen
Input Range $0-20 \quad$ Tolerance $2 \%$ - ovtput

Units: $A$

$\begin{array}{ccccccccc}\operatorname{Input}(A) & \text { Output(ma)(MicoN) } & \text { Lo: } & \text { Hi: } & \text { As Found } & \text { In/Out } & \text { As Left } \\ 0 & 4.00 & 0 & 3.68(-1) & 4.32(-1) & 4.00(0) & \text { N } & 4.00 & (0) \\ 20 & 20.00 & 20 & 19.68(19) & 20.32(21) & 20.00(20) & \text { IN } & 20.00(20)\end{array}$

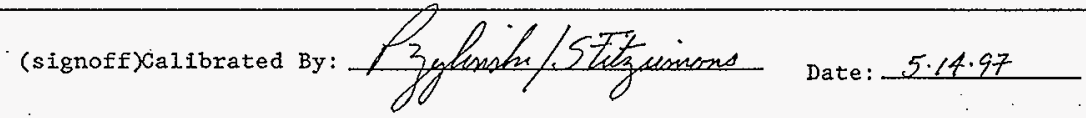

Location: $10 Z$ AZ COOLNG WATER TOWIR (PuMP 1B)

comments. Factory calibrated; check zero; sPAN. Use current generator w/ calibrated MTE clamp on meter functional 
Tag: IALL_AZ2EWT_1

Cntlr: 53

Chan No: 19

P\&ID: $\mathrm{H}-2-1310 \% 0$

Logic:

Remarks :

Location:

Actuation Method: TURNED LEAK DETECTDR ON AND OFF

sensor calibration: $N / A$

口

per procedure (data attached)

Setpoint:

Loop Tolerance:

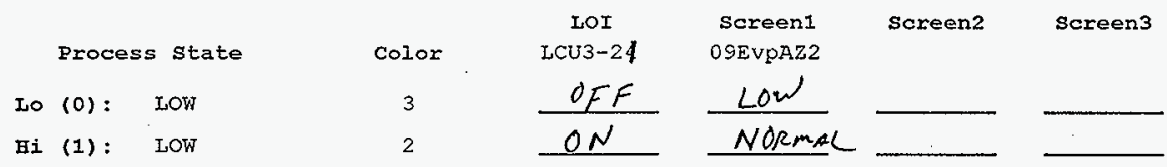

\section{Annunciator(s): $\quad$ Priority}

Hi. 0

Lo: 2
Alarm color (actual)

yeccow

other:

MTE Instr. No:

Due Date:

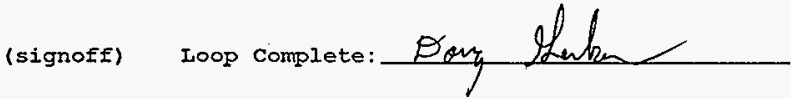


$3 / 28 / 96$

W-030 LOOP TEST DATA SHEET

HNF-SD-W030-TD-003,

REV. 0, PAGE 218

Tag: IAL_AZ2_EWTK_1 I/O TYpe: DI Description: Az2EWTWrLvILo

Cutler: 53 Chan No: 23 P\&ID: H-2-131070. Logic:

Remarks :

Location:

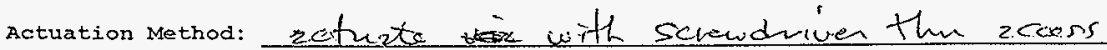

port praised

Sensor Calibration:

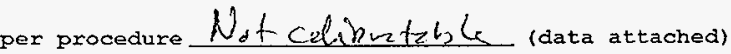

(signoff)

Setpoint:

Loop Tolerance:

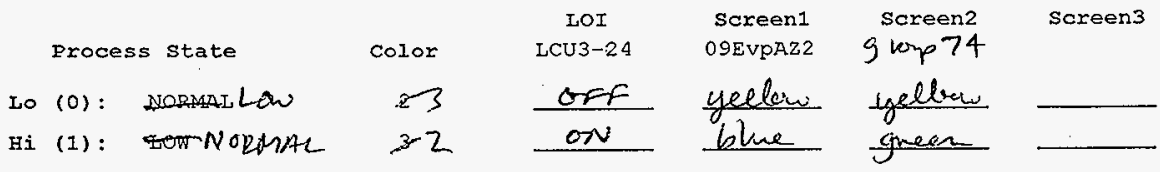

$\begin{array}{rcc}\text { Annunciator (s): } & \text { Priority } & \text { Alarm color (actual) } \\ \text { Hi: } & 20 & - \\ \text { Lo: } & 62 & \text { yellow }\end{array}$

- revised per KEA

Other:

MTE Instr. No:

Due Date:

(signoff) Loop complete:

Ariel

Date: $4 / 17 / 46$ 
2/19/97

W-030 CALIBRATION DATA SHEET

HNF-SD-W030-TD-003,

REV. O, PAGE 219

Tag: LSL-AZ102EWTK-1

P\&ID: H-2-131070, SH 1

LOOP: H-2-131334, SH 16

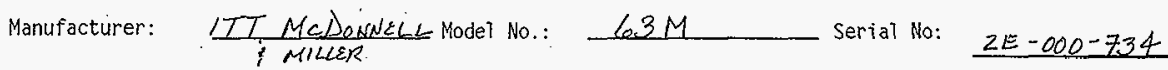

MTE Instr. No: _-N/D

Due Date: $\quad-N / /$

Input Range Functional

Tolerance $N / A$

Units: Switch

Input output

Mech SWITH.

Lo: Hi: As Found In/ Out As Left

TrIP.

"SEE INSTRUCTIONS IN COMMENTS"

(signoff) Calibrated By:

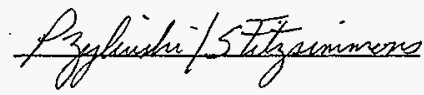

Date: $\quad 4 \cdot 18 \cdot 97$

Location: 10Z AZ Cooling WATER Tower.

Comments

Instructions: Actuate level switch with screwdriver through access port provided. Verify $\angle A L$ (yellow) recieved
on Micon screen.

IS ALARM Received at MICON $Y{ }_{N}$ 
June 4, 1996

W030 LOOP TEST DATA SHEET

HNF-SD-W030-TD-003,

REV. 0, PAGE 220

G: CE/CSH-AY102EWT-1 I/0 Type: N/A

Description: Tower Gond.

P\&1D: $H-2-131068$.

Remarks: LoCal] loop on AY102 Cooling Tower with no input to the MCS. Controls conductivity of the tower water via feed and bleed.

Location: Conductivity element in cooling tower filtration 100p. Conductivity switch/controller located in panel next to local tower control panel

Actuation Method: Simulate conductivity changes with a decade box and verify that the controller will open the tower drain valve on high conductivity and close the drain valve when conductivity is below setpoint $(1000$ ambo $)$

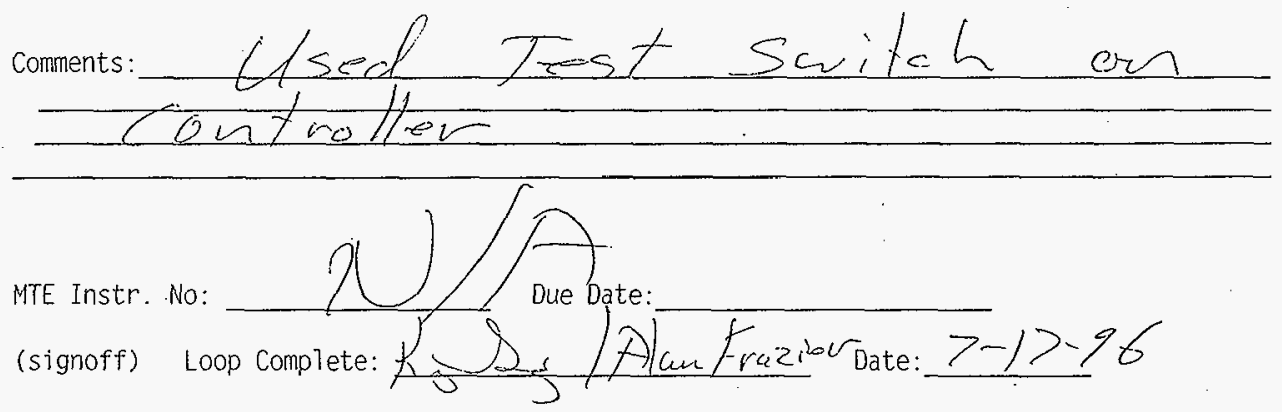


Tag: CSH-AY102EWT-1

P\&ID: $H-2-131068, \mathrm{SH} 1$

LOOP: W-030-P1

Manufacturer: IDEX_.. Model No.: SHICEZACX Serial No: $5 F-72 L$ MTE Instr. No: Butfer soln. s/w 7070 Due Date: $2 / 98$ Limitel cal.

Input Range $0-6000 \quad$ Tolerance $2 \%$

Units: umho's/cm

Input output . Lo: Hi: As Found In/Out As Left

7.1:2 Pane Meter Reading _n N/A - initial cal 6,000

\begin{tabular}{l|lll}
7.1 .6 & Test Meter Pan water reading $* 4,500$ m mos/cm & 4,500 \\
7.2 & Electrode Cleaned/Replaced & No
\end{tabular}

7.3 Specified Trip point Setting: 1000 umhos/em

7.3.2 As found Trip point Panel meter reading $N / A$

7.4.2 Control indicator Light Panel Meter reading 200 inehos

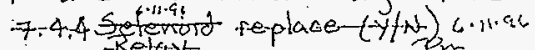

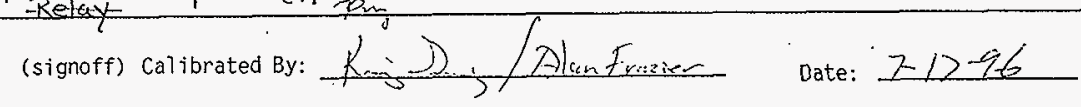

Location: AY Coling water towers

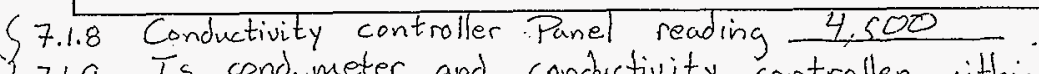

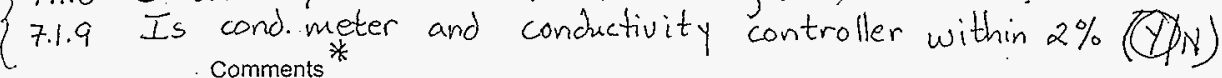

7.4 .5 Is the reading from 7.4 .2 withis $6 \%$ of specified Trip Point setting? $(Y / N)$ 
P\&IO:H-2-131070

Remarks: Local 100p with no inputs to the MCS. Interupts conductivity controls if there is no flow established through the conductivity cell.

Location: Flow switch located at AZ102 cooling tower in filtration loop downstream of conductivity element.

Actuation Method:

Actuate switch locally or simulate switch actuation using electrical jumper. Verify that a no flow condition prevents the conductivity cell from acutating the tower drain valve on high conductivity.

Comments:

Buffer suln s/N7070

MTE Instr. No: Af Rngulas:se Date:

(signoff) Loop Complete:

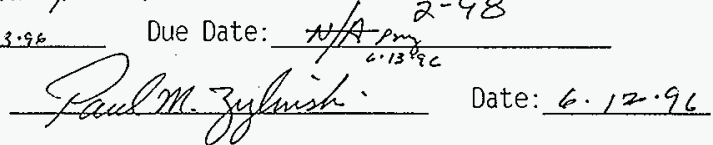


$5 / 1 / 97$

W-030 CALIBRATION DATA SHEET.

HNF-SD-W030-TD-003,

REV. 0, PAGE 223

Tag: FSL-AZ102WS-1

P\&ID: H-2-131070, SH 1

LOOP:

Manufacturer: Idex/Pulsafeeder Model No.: $\quad$ Option 3 Serial No: $5 F \neq 27$

MTE Instr. No:

Functional

Due Date: $\frac{N / A}{7 / A}$

Input Range

Units:

Input Output
Tolerance

Lo:
As Found
In/Out

Functional

(signoff) Calibrated By:

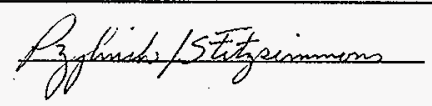

Date: $5 \cdot 29 \cdot 97$

Location: $102 A Z$ Sorting hate tower

Comments * De-energize cir pump to cause low flow.

Does flow light on Conductivity meter (CSH-AZ1OZEWT-1) light up when flow switch is activated? (Y) $N$

* Manually activated while system is siD and drained. 
Tag: TC-AZ102EWT-1A2

P\&ID: $H-2-131070$. SH 1

LOOP:

Manufacturer: JoHusan Controls Model No.: ABUABA-44 Serial No: 2E-000-704. $817 \cdot 13 \cdot 55 \cdot 015$

MTE Instr. No: $\quad 817 \cdot 45 \cdot 08 \cdot 040$ $817 \cdot 13 \cdot 35 \cdot 025$

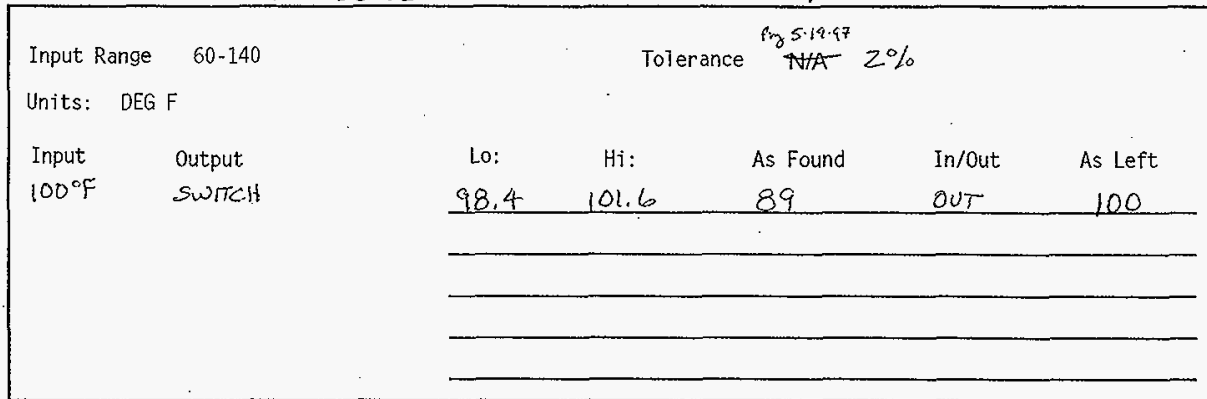

(signoff) Calibrated By:

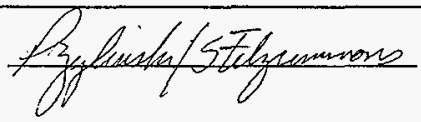

Date: $5 \cdot 19.97$

Location: IOZAZ COOLING WATER TOWER

Comments Damper control, set to 'B' span for testing. - begins opening
damper at $100^{\circ} \mathrm{F}$ CALIBRATE PER VENDOR DATA SET TO TURN ON AT 100 DEG F 
$3 / 8 / 96$

W.030 CALIBRATION DATA SHEET

HNF-SD-W030-TD-003,

REV. 0, PAGE 225

TC-AZIOZEWव-IAO

$4-2-131070,5 H 1$

Tag: IE-A210IETTAZ

P\&ID: H-2-131069,-SH-1 LOOP:

$$
\begin{aligned}
& 2 E-000-704 \\
& 2 E-000-703
\end{aligned}
$$

Serial No:

MTE Instr. No: $817-45-08-040$

Due Date: $2=5-97$

Input Range 60-140

Units: $D E G F$

Input Output

$100^{\circ} \mathrm{F}$ Switch

Model <compat>No.: $A B \not A B A-44$

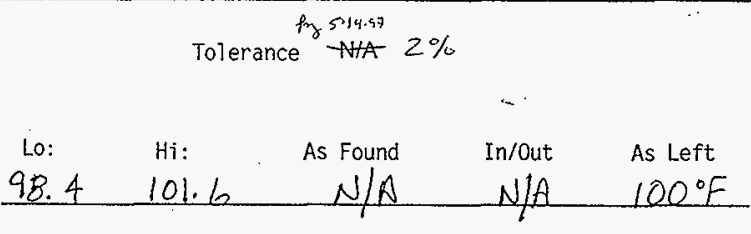

(signoff) Calibrated By:

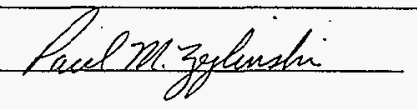

Date: $6-3 \cdot 96$

Location: 102 AZ Cooling water tower

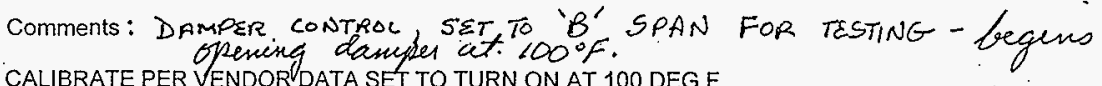
CALIBRATE PER VENDORLATA SET TO TURN ON AT 100 DEG F
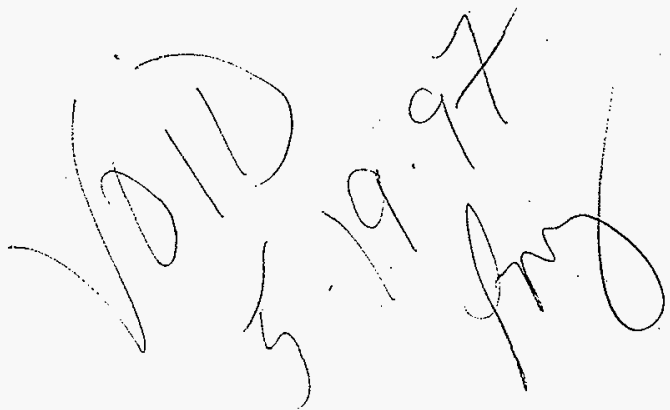
$6 / 3 / 96$

W-030 CALIBRATION DATA SHEET

HNF-SD-W030-TD-003,

REV. 0, PAGE 226

TS-AZ102EWT-|A|

Tag: $\forall E-A Z K I-1$
P\&ID: $\begin{aligned} H-2-131070,5 H 1 \\ t-2-231078 r-S H-1\end{aligned}$
LOOP:

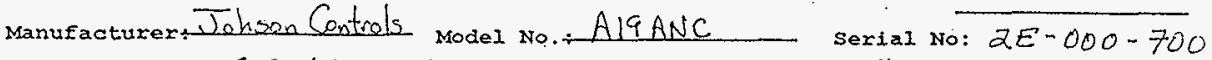
$817-45-08-04$

MIRE Instr. No: $\frac{817 \cdot 13 \cdot 55 \cdot 015}{778-13 \cdot 55-002}$ Due Date: $\frac{2-51 \cdot 97}{10 \cdot 30 \cdot 96}$

Input Range $40^{\circ} \mathrm{F}^{*} 0-150$

Tolerance $2 \%$

Units: ${ }^{\circ} \mathrm{F}$

Input output

$40 \%$ SwitcH

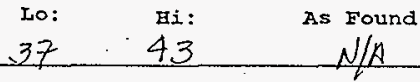

In/ out

NI

As Left

$39^{\circ} \mathrm{F}$

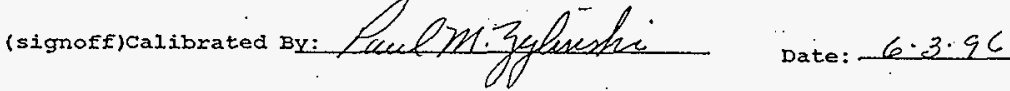

Location: $102 \mathrm{AZ}$ COONS WATER TOWER.

Comments Low TEMP. SWITCN, switch closes on $40^{\circ} \%$ to energize heaters.

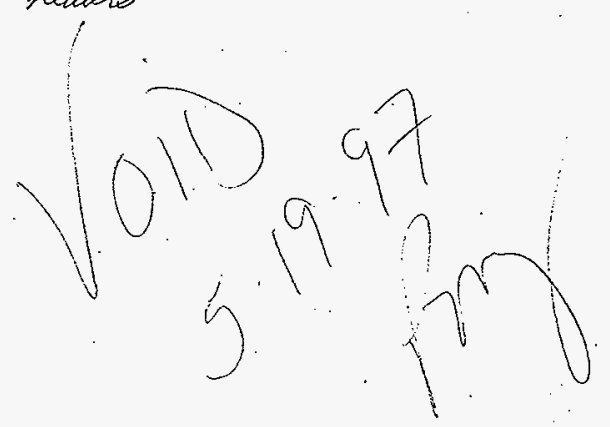


Tag: TS-AZ102EWT-1A1

P\&ID : H-2-131070, SH 1

LOOP:

Manufacturer: Johnsan Controls ModeI No.: AIGANC $817 \cdot 13 \cdot 55 \cdot 015$ MTE Instr. No: $817 \cdot 4 \cdot 5 \cdot 083 \cdot 040$ $\frac{817 \cdot 45 \cdot 085 \cdot 043}{817 \cdot 35: 025}$ Due Date: $\frac{5 \cdot 21 \cdot 97}{7 \cdot 28 \cdot 98}$ Sexial No: $2 E-000-700$

Input Range $0-150$ Tolerance $2 \%$

Units: DEG F

$\begin{array}{llccccc}\text { Input } & \text { Lutput } & \text { Lo: } & \text { H1: } & \text { As Found } & \text { In/Out } & \text { As Left } \\ 40 & \text { SwitcH } & 37 & 43 & 44 & \text { ovT } & 40\end{array}$

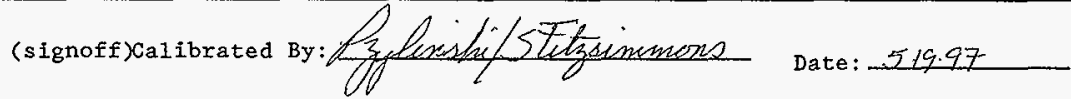
Location: $102 \mathrm{AZ}$ COOLNG WATER TOWER

Comments Low TEMP SWITCH, SWITCH CLOSES ON $40^{\circ} \mathrm{F}+$ TO ENERGIZE HEATERS CALIBRATE PER VENDOR DATA SET TO TURN ON AT 40 DEG F 
Tag: YS_AY1_EWSP_1 I/O TYPe: DI

Description: AY1EWSpyPumpsts
CntIr:
47
Chan No:
21
PEID: $\mathrm{H}-2-131067$
Logic:

Remarks :

Location:

pullod in stuten to openzie bzelc contef

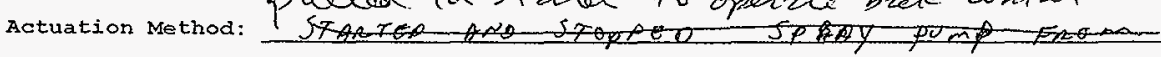

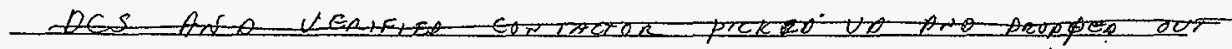
Sensor calibration: $N / A$

$\square \ldots$ per procedure (data attached)

(signoff)

Setpoint:

Ioop Tolerance:

Process state
Lo $(0):$ Color

Annunciator(s):

Priority

Alarm color (actual)

$\begin{array}{ll}\text { Hi: } & 0 \\ \text { Io: } & 0\end{array}$

other:

MTE Instr. No:

Due Date:

(signoff) Loop complete:

$$
\text { Letested }
$$

Date: $4 / 15 / 96$

$5 / 2 / 96$ 
Cntir: 47

Remarks :

Location:

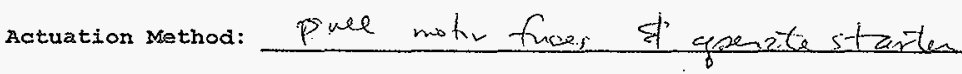

\section{Sensor Calibration:}

a $\ldots$ pex procedure $N / A$ (data attached)

Setpoint:

Loop Tolexance:

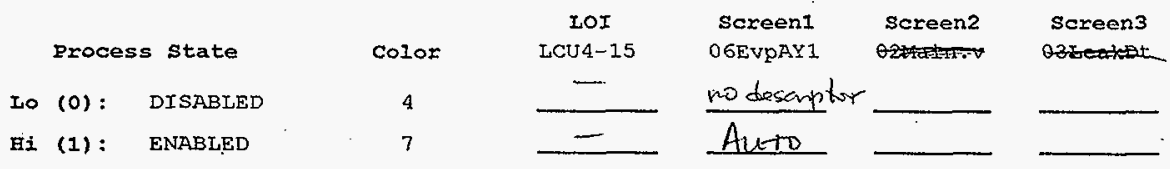

$\begin{array}{rcc}\text { Annunciator(s): } & \text { Priority } & \text { Alarm color (actual) } \\ \text { Hi: } & 0 & \\ \text { Io: } & 0 & \end{array}$

other:

MIE Instr. No:

Due Date:

(signoff) Loop complete: 
Tag: YS_AY1_EWP_1A1 I/O TYPe: DI

Cntir:

Chan No: 25

25

Description: AYIEWPmpA RemLoC

Remarks:

Location:

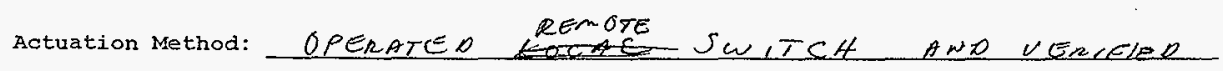
DCS GRARALC

Sensor Calibration: $N / A$

D__ per procedure (data attached)

Logic: 
Tag: YS_AY1_EWP_1BI I/O Type: DI

Description: AY1EWPMpB RemLoc

Cotlr:

47

Chan No: 26

P\&ID : $\mathrm{H}-2-131067$

Logic:

Remarks :

Location :

Actuation Method:

o perzated remote

\section{GRAPAIC}

Sensor calibration: $N / A$

per procedure

(data attached)

(signoff)

setpoint:

Loop Tolerance:

$\begin{array}{lccc}\text { Process state } & \text { Color } & \begin{array}{c}\text { LOI } \\ \text { ICU4-17 }\end{array} \text { O6EvpAY1 } & \text { Screen2 } \\ \text { Lo (0): LOCAL } & 7 & \text { STATE } & \text { STATE }\end{array}$

Annunciator (s):

Priority

Hi:

Lo:

0

3
Alarm color (actual)

w ito

\section{Other:}

MTE Instr. No:

Due Date:

(signoff) Loop complete: D.\&.

Date: $4 / 15 / 96$ 
Tag: AY1 EWP_1BA

Cntir:

Logic :

Remarks :

Location:

Actuation Method:

ACTIUATED SWITCH AT DCS ANO UENUEE

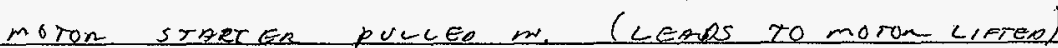

Sensor Cajibration: $N / A$

$\square$ per procedure (data attached) (signoff)

Setpoint:

Loop Tolerance:

Process State

Lo (0): FALSE

Hi (I) : TRUE

Annunciator (s) :

Hi:

LO:
Priority

0

0
LOI

Color

4

7
Screen1 Screen2

Scxeen 3 
Tag: AY1 $E W P \_1 B B$

Cntlx:

Remarks :

Location:

Actuation Method:

ACT,U ATEO SWITCH Q5

DCs

END $\triangle E R I E N O$

morom srowten Parjopep

Sensor Calibration: $N / A$

$\square$

per procedure

(data attached)

Setpoint :

Loop Tolerance:

Process state

Lo (0): FALSE

Hi (1) : TRUE
IOI

Color

4

7
Screen1 Screen2

Screen3

$\begin{array}{rcc}\text { Annunciator (s) : } & \text { Priority } & \text { Alarm Color (actual) } \\ \text { Hi: } & 0 & \\ \text { Lo: } & 0 & \end{array}$

other:

MTE Instr. No: Due Date: 
Tag: AY1_EWSP_1A I/O TYpe: DO

Cnt1r: 49 Chan No: 9 P\&ID: H-2-131067,5H.1 Logic:

Remarks :

Location:

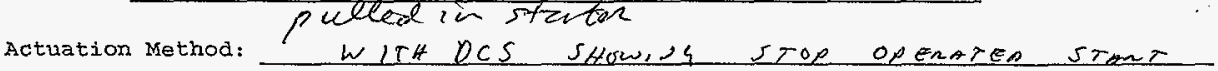

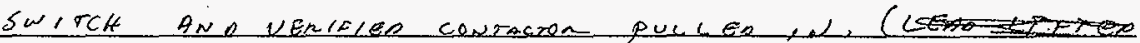

Sensor Calibration: $N / A$

$\left(\begin{array}{l}\text { PULLEO cONTECrON } \\ \text { PUSES }\end{array}\right.$

$\square$

per procedure

(data attached)

(signoff)

Setpoint:

Loop Tolerance:

Process state

Lo $(0):$ FALSE

Hi $(1):$ TRUE
LOI

Color

4

7
Screen 1 $O E[p A Y$
Screen2

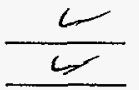

Screen 3

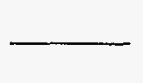

Annunciator (s):

priority

Hi:

Lo:

0

0

Alarm Color (actual)

Other:

MTE Instr. No: Due Date:

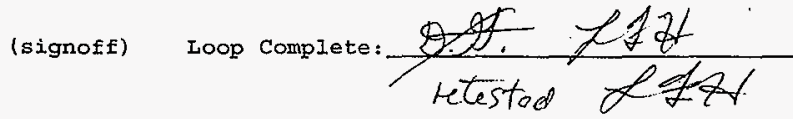

Date: $4 / 15 / 46$ $5 / 2 / 96$ 
Tag: $A Y I$ EWSP_18 I/O. Type: DO Description: EwSpPump Stop

Cnt1r:

49 Chan No: 10

P\&ID: $H \cdot 2-131067,5 H .1$

Logic:

Remarks :

Lociation:

Actuation Method:

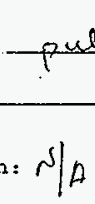

per procedure (data attached)

(signoff)

Setpoint:

Ioop Tolerance:

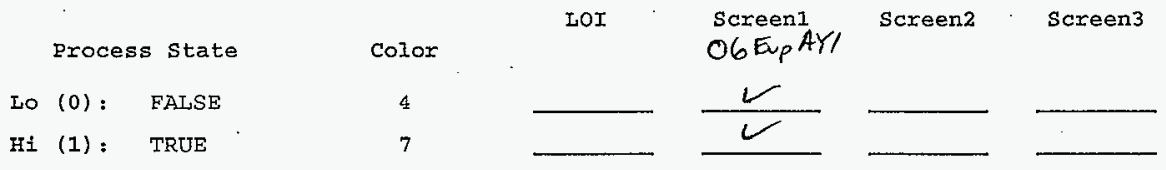

$\begin{array}{rcc}\text { Annunciator (s): } & \text { Priority } & \text { Alarm Color (actual) } \\ \text { Hi: } & 0 & \\ \text { LO: } & 0 & \end{array}$

Other:

MTE Instr. No: 
Tag: AYI__EWT_1A I/O TYPe: DO Description: EWTOWer On

Cntlx: $49^{\circ}$ Chan No: 11 P\&ID:H-2-131067, SH.1 Iogic:

Remarks :

Location:

Actuation Method: $\quad 2$.

Sensor Calibration:

$\square$ …...... per procedure

$N / A$ (data attached)

Setpoint:

Ioop Tolerance:

Process State
LO (0): Color

$\begin{array}{rcc}\text { Annunciator }(s): & \text { priority } & \text { Alarm Color (actual) } \\ \text { Hi: } & 0 & \\ \text { LO: } & 0 & \end{array}$

other:

MTE Instr. No:

Due Date:

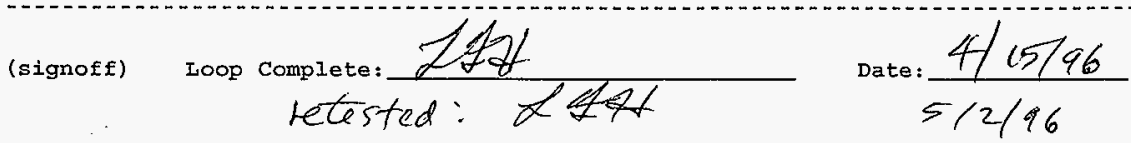


Tag : AY1 EWT_1B

Cntlr:

49. Chan No: 12

P\&ID: $H \cdot 2 \cdot 131067, S H .1$ Logic:

\section{Remarks :}

Location:

Actuation Method: operatad stater

Sensor Calibration:

$\square$ per procedure

N $12 A$ (data attached)

Setpoint :

Ioop Tolerance:

\begin{tabular}{|c|c|c|c|c|c|c|c|}
\hline & Process & s state & Color & LOI & $\begin{array}{l}\text { screen } 1 \\
\text { OGEup AYI }\end{array}$ & Screen2 & Screen 3 \\
\hline Lo & $(0):$ & FALSE & 4 & & $L$ & & \\
\hline $\mathrm{Hi}$ & (1): & TRUE & 7 & & & & \\
\hline
\end{tabular}

$\begin{array}{rcc}\text { Annunciator }(\mathrm{s}): & \text { Priority } & \text { Alarm Color (actual) } \\ \text { Hi: } & 0 & \\ \text { LO: } & 0 & \end{array}$

Other:

MTE Instr. No:

Due Date:

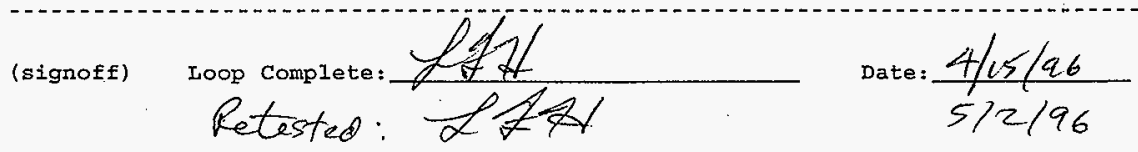


$3 / 28 / 96$

W-030 LOOP TEST DATA SHEET

HNF-SD-W030-TD-003,

REV. 0, PAGE 238

Tag: YS_AY2_EWSP_1 I/O Type: DI Description: AY2EWSpYPumpSts

Contr: 48 Chan No: 21 P\&ID: H-2-131068 Logic:

Remarks:

Location:

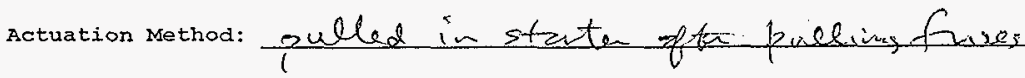

Sensor Calibration:

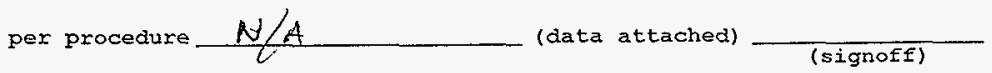

Setpoint:

Loop Tolerance:

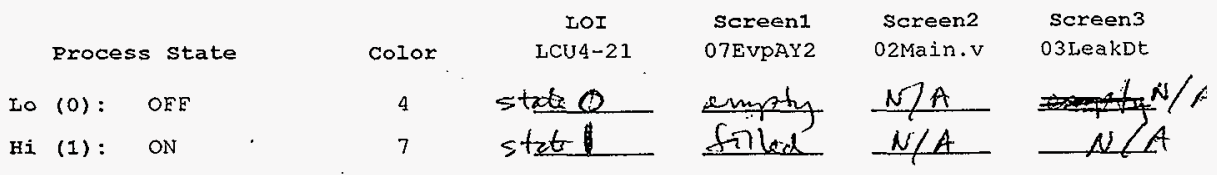

$\begin{array}{rcc}\text { Annunciator }(s): & \text { Priority } & \text { Alarm Color (actual) } \\ \text { Hi: } & 0 & \\ \text { Lo: } & 0 & \end{array}$

other:

Also totals scram $-N / A$

MTE Instr. No:

Due Date:

(signore) Loop complete:
Retested:

Date: $\frac{4 / 18 / 96}{5 / 2 / 96}$ 
Tag: YS_AY2_EWT_1 I/O Type: DI . Description: AY2EWTwrEanEnable

CntIr: 48 Chan No: 22 R\&ID: H-2-131068 Logic:

Remarks :

Iocation:

Actuation Method:

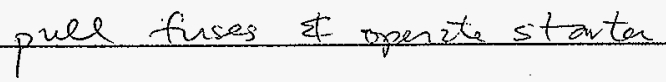

Sensor Calibration:

$\square$ per procedure $\ldots N / 4$ (data attached) (signoff)

Setpoint:

Loop Tolexance:

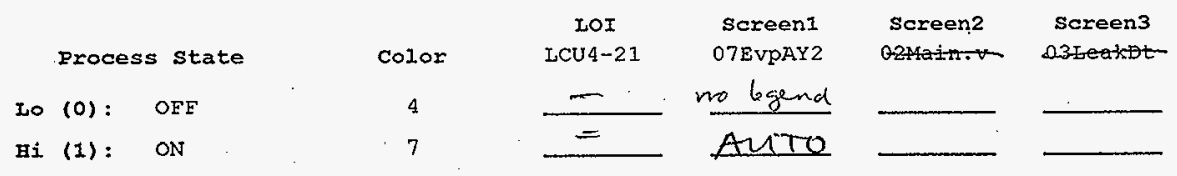

$\begin{array}{rcc}\text { Annunciator(s): } & \text { Priority } & \text { Alarm Color (actual) } \\ \text { Hi: } & 0 & \\ \text { IO: } & 0 & \end{array}$

other:

MTE Instr. No:

Due Date:

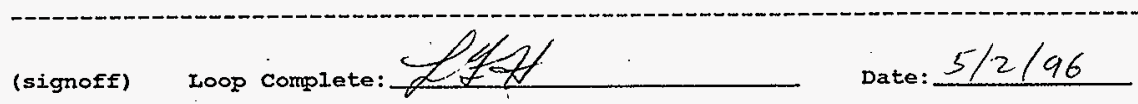


Tag: YS_AY2_EWP_1AI I/O TYpe: DI Description: AY2EWPmpA RemLoc

Cntle:

48 Chan No: 25

P\&ID: $H-2-I 31068$

Logic:

Remarks :

Iocation:

Actuation Method:

suital

Sensor calibration:

$\square$

per procedure _.. $N / A$

(data attached)

(signoff)

Setpoint:

Loop Tolerance:

\begin{tabular}{|c|c|c|c|c|c|c|c|}
\hline \multirow{2}{*}{\multicolumn{2}{|c|}{ Process }} & \multirow[b]{2}{*}{ state } & \multirow[b]{2}{*}{ Color } & IOI & Screen1 & & \multirow{2}{*}{ Screen 3} \\
\hline & & & & $\operatorname{LCU} 4-22$ & O7EvpAY2 & Gore 52 & \\
\hline Lo & $(0):$ & LOCAL & & & white & $\angle 0 C A+2$ & \\
\hline $\mathrm{Hi}$ & (1): & REMOTE & 2 & iftete 9 & - & $M A N U$ & \\
\hline
\end{tabular}

$\begin{array}{rcc}\text { Annunciator(s): } & \text { Priority } & \text { Alarm color (actual) } \\ \text { Hi: } & 0 & \text { Lo: } \\ \text { Loite }\end{array}$

other:

MTE Instr. No:

Due Date:

(signoff) Loop complete:

Date: $4 / 18 / 96$ 
Tag: YS_AY2_EWP_1B1 I/O TYPe: DI

Description: AY2EWPmpB RemLoc

Cntlr:

48

Chan No: 26

P\&ID: $H-2-131068$

Logic:

Remarks :

Iocation:

Actuation Method:

Sensor Calibration:

$\square$ per procedure

$$
\text { NA }
$$
(data attached) (signoff)

Setpoint:

Loop Tolerance:

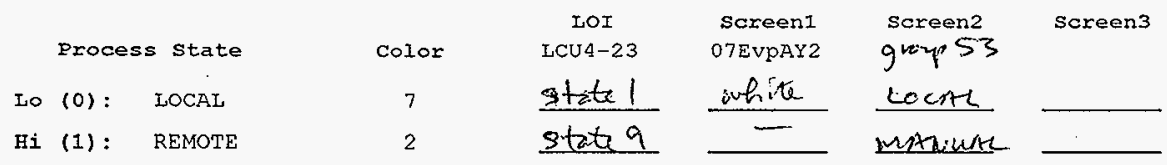

Annunciator(s): $\quad$ Priority

Hi :

Io:

3
Alarm color (actual)

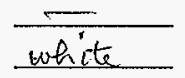

other:

MTE Instr. No: Due Date: 
Tag: AY2___EWT_1A I/O Type: DO Description: EwTowex On

CntIr: 49 Chan No: 26 R\&ID:H-2-131068, SH.1 IOgic:

Remarks :

Iocation:

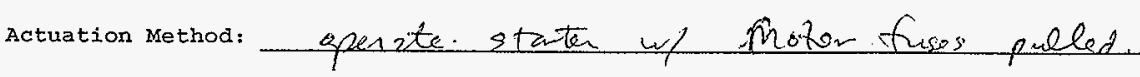

Sensor Calibration:

$\square$...... per procedure

1. 4 (data attached)

Setpoint:

Loop Tolerance:

Process state

IO $(0):$ FAISE

Hi (1) : TRUE
LOI

Color

4

7
Screen1 Screen2

Screen3

$\begin{array}{rcc}\text { Annunciator }(s): & \text { Priority } & \text { Alarm Color (actual) } \\ \text { Hi: } & 0 & \\ \text { Lo: } & 0 & -\end{array}$

other:

MTE Instr. No:

Due Date:

(signoff) roop complete: 
Tag: AY2__EWT_1B I _ IO Type: DO

Description: EwTower Off

Cotix:

49 Chan No: 27.

P\&ID : H-2-131068, SH.1

Logic :

Renarks :

Iocation:

Actuation method: pull motor fuses it epenzte staten

Sensor Calibration:

[

(data attached) attached)

Setpoint :

Ioop Tolerance:

Process State

LO $(0)$ : FALSE

Hi (1): TRUE
LOI

Color

4

7 (data attached) attached)

Annunciator (s) : $\quad$ Priority

Hi :

Io:
0

0

(a)

Alarm Color (actual)

Other:

MTE Instr. No:

Due Date:

(signoff) Loop complete: Cetested:

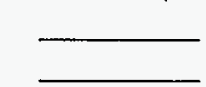


Tag : AY2 EWSP 1 A

Cotir:

Logic:

\section{Remarks:}

Location

Actuation Method: priel fuses of senente strater

Sensor Calibration:

$\square$ per procedure (data attached)

Setpoint :

Loop Tolerance:

Process State

Lo (0): FAISE

Hi (1) : TRUE

Annunciator (s): Hi : LO:
IOI

Color

7

\section{4 \\ 4}

Screen1

Screen2

\section{Screen 3}


CntIr: 49 Chan No: 25

PEID: $H-2-131068$, SH. I Logic:

Remarks :

Iocation:

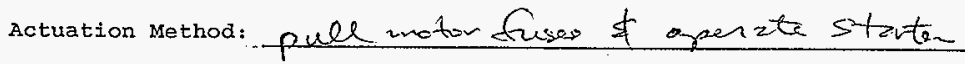

Sensor Calibration:

$\square$ per procedure (data attached)

Setpoint :

Ioop Tolerance:

\begin{tabular}{|c|c|c|c|c|c|c|c|}
\hline & Process & state & Color & LOI & Screen1 & Screen2 & Screen 3 \\
\hline Lo & $(0):$ & FALSE & 4 & & & & . \\
\hline $\mathrm{Hi}$ & (1): & TRUE & 7 & & & & \\
\hline
\end{tabular}

$\begin{array}{rcc}\text { Annunciator (s): } & \text { Priority } & \text { Alarm Color (actual) } \\ \text { Hi: } & 0 & \\ \text { LO: } & 0 & \end{array}$

Other:

MTE Instr No: Due Date:

(signofe) Loop complete: flay

Petroted: Date: $\frac{4 / 18 / 96}{5 / 2 / 96}$ 
Tag: AY2_EWP IAA I/O TYPe: DO

Description: EwPumplA Start

Cntlx:

49 Chan No:20

PEID: $H-2-131068$, SH. I Logic:

Remarks:

Iocation :

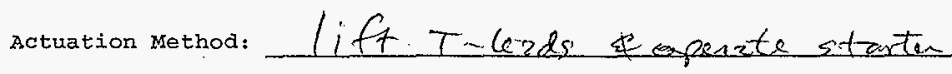

Sensor Calibration:

$\square$ per procedure (data attached)

Setpoint:

Loop Tolerance:

Process State
TO $(0):$ COIOI

Annunciator (s):

$\begin{array}{ll}\text { Hi: } & 0 \\ \text { Io: } & 0\end{array}$

Other:

MTE Instr. No:

Due Date:

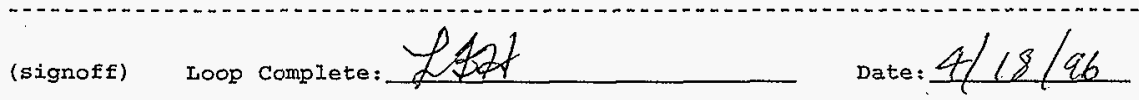


Tag : AY2 EWP_1AB I/O TYpe: DO

Description: PvRstrtB Stop

Cntlr:

49 Chan No:21

P\&ID: H.2.131068, SH. I Logic:

Remarks:

Location:

Actuation Method: lift $T=$ ceds $\$$ openate storter

Sensor Calibration:

$\square$ per procedure (data attached)

(signoff)

Setpoint:

Loop Tolerance:

\begin{tabular}{|c|c|c|c|c|c|c|c|}
\hline & & & & IOI & Screen1 & Screen2 & Screen 3 \\
\hline & Process & s state & Color & & & & \\
\hline Io & $(0):$ & PALSE & 4 & & -0 & 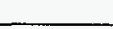 & 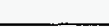 \\
\hline $\mathrm{Hi}$ & (1): & TRUE & 7 & & & & \\
\hline
\end{tabular}

$\begin{array}{rcc}\text { Annunciator }(s): & \text { Priority } & \text { Alarm Color (actual) } \\ \text { Hi: } & 0 & \\ \text { Lo: } & 0 & \end{array}$

\section{other:}

MTE Instr. No:

Due Date:

(signoff) Ioop complete: 121 Date: $\frac{4 / 18 / 96}{6}$ 
Tag: AY2__EWP_1BA I/O Type: DO

Description: EwPumplB Start

CntIr:

49 Chan No: 22

P\&ID : $H-2-131068,3 H$

Logic:

Remarks:

Location:

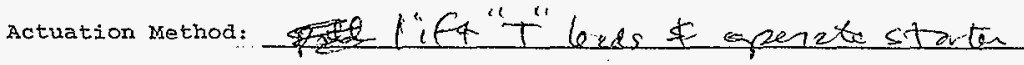

Sensor Calibration:

$\square$ per procedure (data attached)

(signofe)

Setpoint :

Loop Tolerance:

Process state

LO (0): FAISE

Hi (1) : TRUE
IOI

color

4

7
ScreenI Screen2

Screen 3

$\begin{array}{rcc}\text { Annunciator }(s): & \text { Priority } & \text { Alarm Color (actual) } \\ \text { Hi: } & 0 & \\ \text { Io: } & 0 & \end{array}$

Other:

MTE Instr. No:

Due Date:

(signoff) Loop complete:

Date: $4 / 18 / 26$ 
Tag: $A Y 2$ EWP_1BB

CntIr:

Logic:

\section{Remarks :}

Iocation :

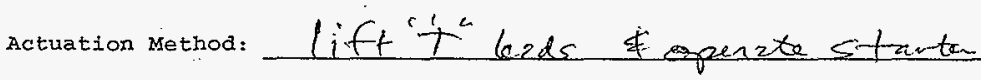

Sensor Calibration:

$\square$ per procedure (data attached)

\section{Setpoint :}

Loop Tolerance:

Process State
LO (0): Color
Hi (1): FALSE

$\begin{array}{rcc}\text { Annunciator (s): } & \text { Priority } & \text { Alarm Color (actual) } \\ \text { Hi: } & 0 & \\ \text { Lo: } & 0 & \end{array}$

Other:

MTE Instr. No:

Due Date:

(signoff) Inoop complete:

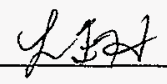

Date: $4 / 18 / 96$ 


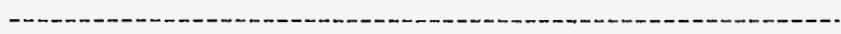

Tag: YS_AZI_EWSP_1 I/O TYpe: DI Description: AZlEWSpyPumpSts

Cntlr: 52 Chan No: 21 P\&ID: H-2-131069 Iogic:

Remarks :

Iocation:

Actuation method: _. pullin stanter

Sensor Calibxation:

$\square$ per prociedure N/A (data attached)

Setpoint:

Loop Tolerance:

\begin{tabular}{|c|c|c|c|c|c|c|c|}
\hline \multirow{2}{*}{\multicolumn{2}{|c|}{ Process }} & & I & LOI & screen 1 & screen 2 & Screen 3 \\
\hline & & s state & Color & ICU3-1.5 & 08EvpAZ1 & 02Main. & 03LeakDt \\
\hline Io & $(0):$ & $O E F$ & 4 & State 0 & $O F F$ & & \\
\hline $\mathrm{Hi}$ & (I): & ON & 7 & STATE 1 & $O N$ & & . \\
\hline
\end{tabular}

$\begin{array}{rcc}\text { Annunciator(s): } & \text { Friority } & \text { Alarm color (actual) } \\ \text { Hi: } & 0 & \\ \text { LO: } & 0 & \end{array}$

other:

MTE Instr, No:

Due Date:

(signoff) Ioop complete: 
Tag: YS_AZI_EWT_1 I/O Type: DI

Description: Az1EWTwrFanEnable

Cntle: 52

Chan No: 22

PEID: $H-2-131069$

Logic:

Remarks:

Location:

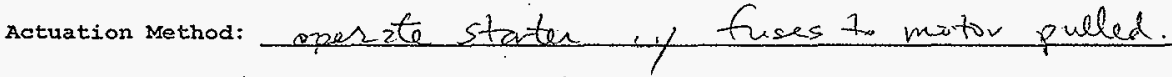

Sensor calibration:

$\square \ldots$ per procedure __. N/A (data attached)

Setpoint:

Loop polerance:

\begin{tabular}{|c|c|c|c|c|c|c|c|}
\hline \multirow{2}{*}{\multicolumn{2}{|c|}{ Process }} & & & IOI & Screen 1 & Screen2 & Screen 3 \\
\hline & & s state & Color & ICU3-15 & 08EvpAZ1 & & \\
\hline Lo & $(0):$ & DISABLED & 4 & stale 0 & no lagend & & \\
\hline $\mathrm{Ei}$ & (1): & ENABIED & 7 & Stat 1 & $A \cup 70$ & & $\therefore$ \\
\hline
\end{tabular}

$\begin{array}{rcc}\text { Annunciator(s): } & \text { Prioxity } & \text { Alarm color (actual) } \\ \text { Ei: } & 0 & \\ \text { Io: } & 0 & \end{array}$

Other:

MIE Instr. No:

Due Date:

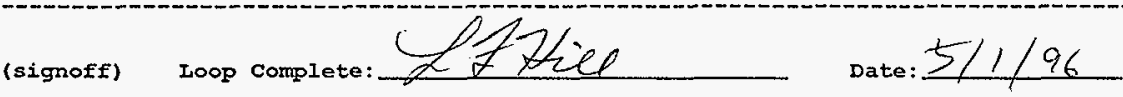


Tag: YS_AZ1_EWP_1AI I/O Type: DI

Description: AZ1EWPmpA RemLOC

Cntlr: 52 Chan No: 25 P\&ID: H-2-131069 Logic:

Remarks :

Location:

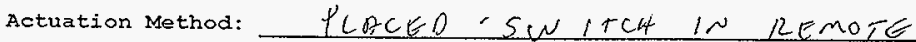

sensor calibration: $N / A$

$\square$ ____ per procedure (data attached)

Setpoint:

Loop Tolerance:

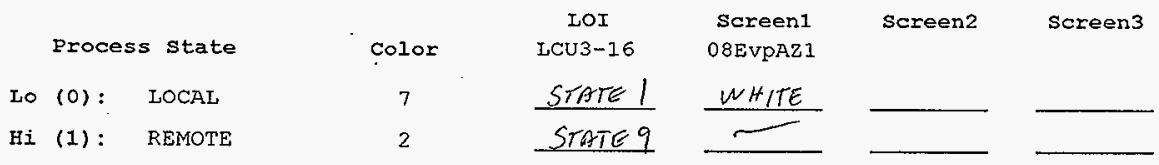

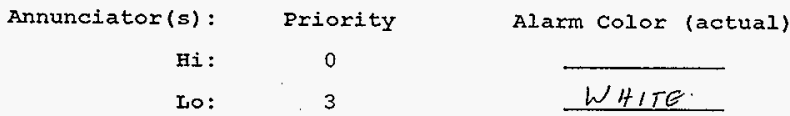

Other:

MTE Instr. No:__nlP Due Date: 
Cnt1r:

52 Chan No:

26

P\&ID: $11-2-131069$

Remarks:

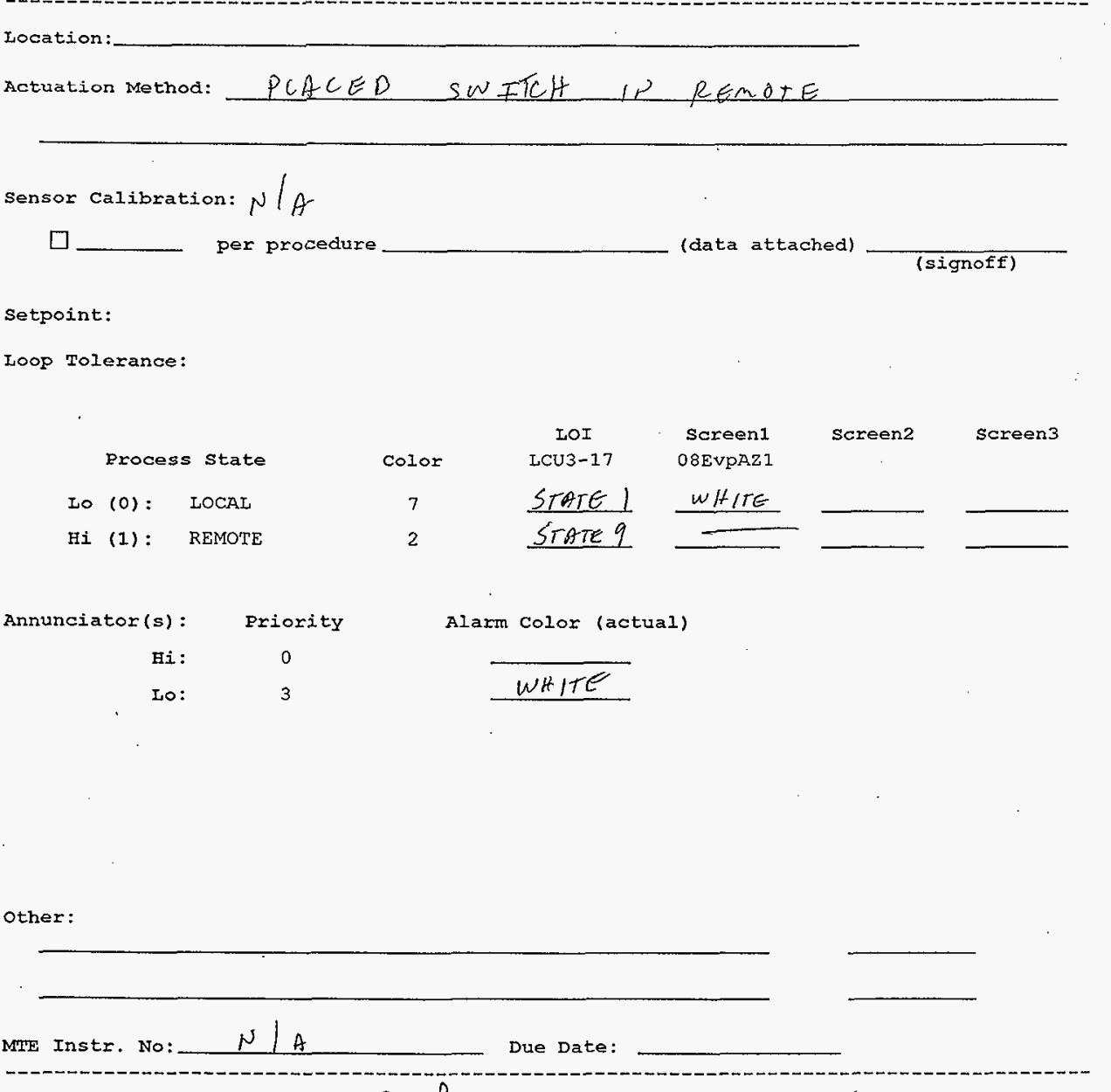

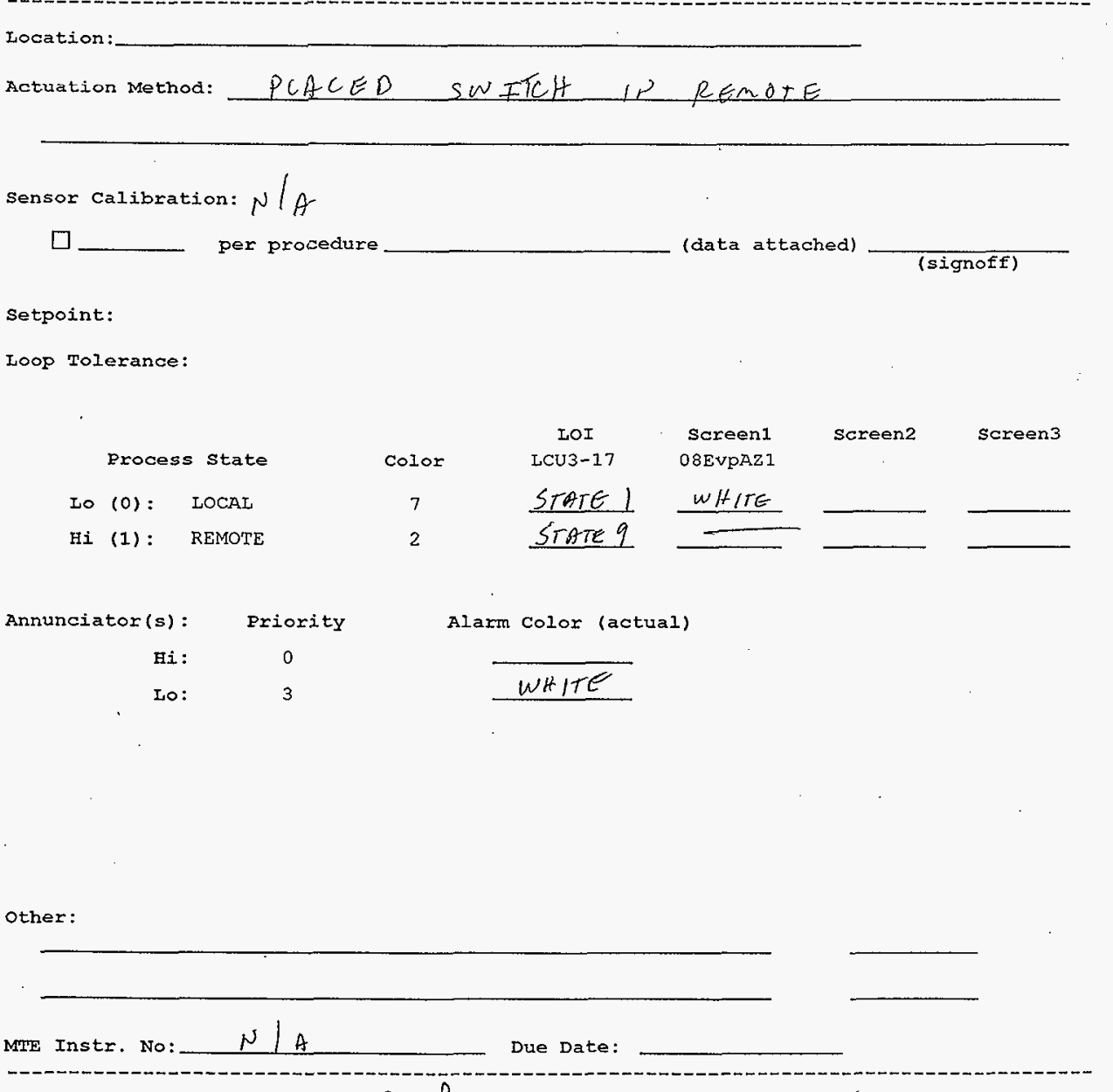

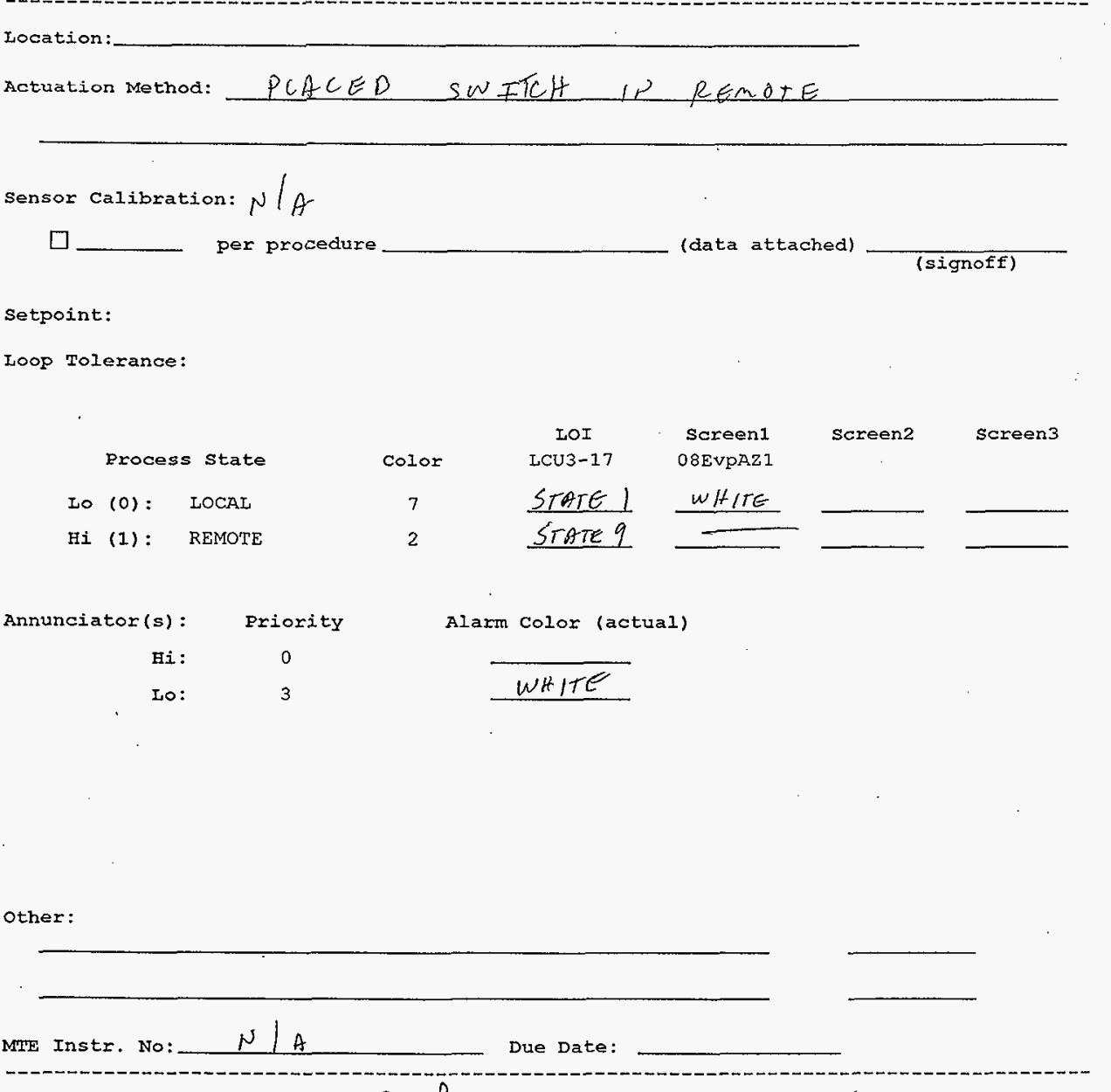

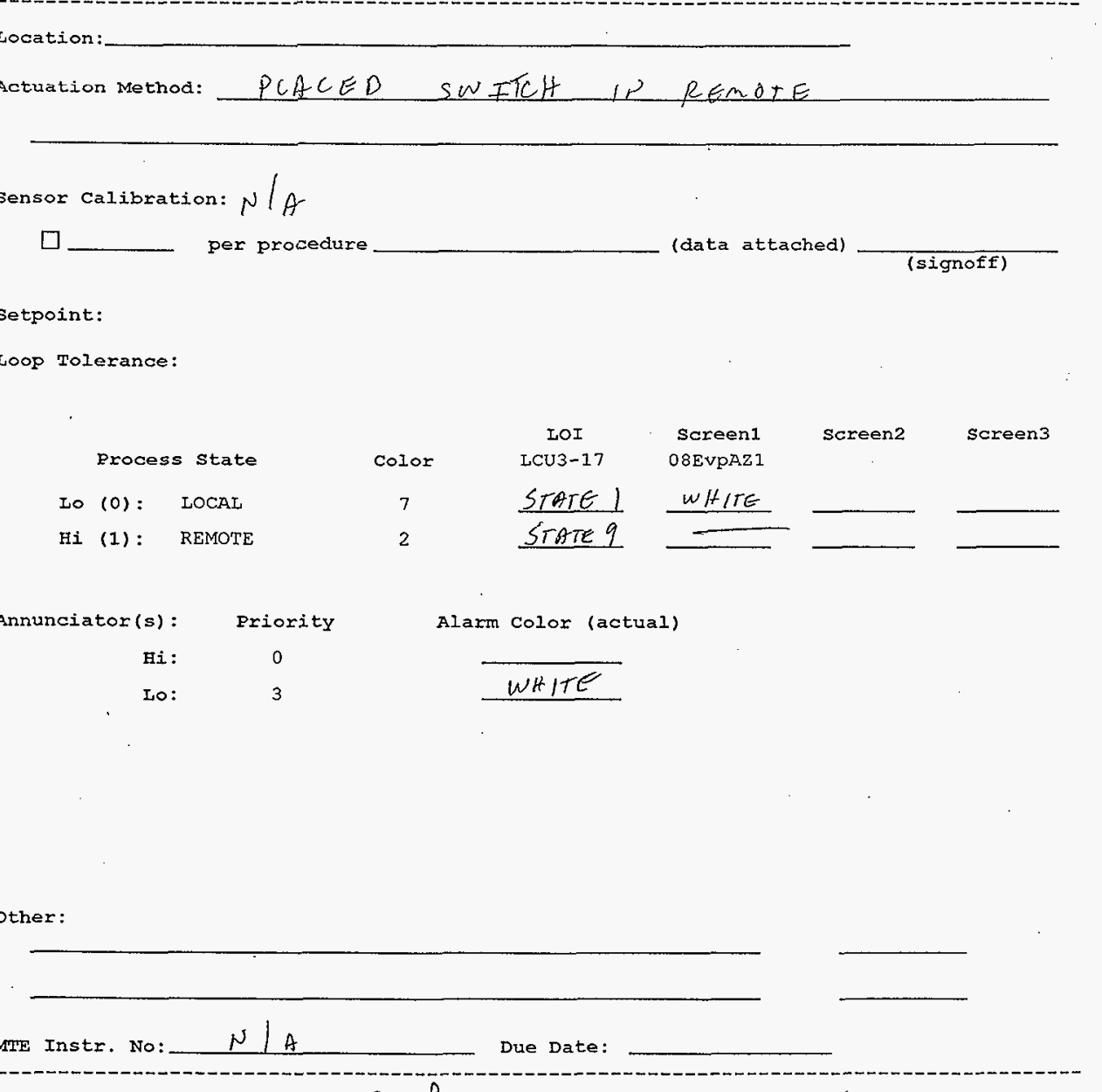

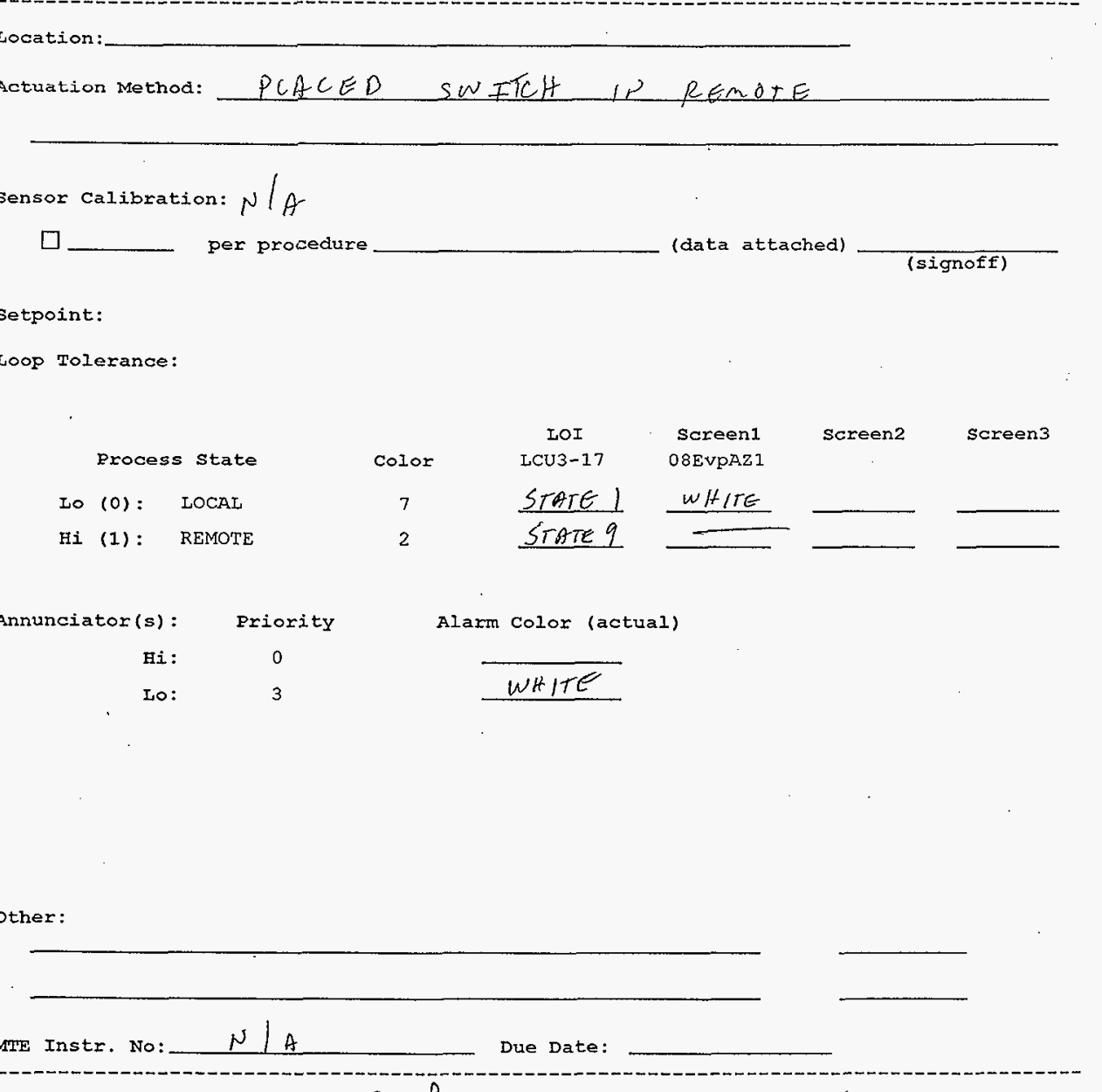

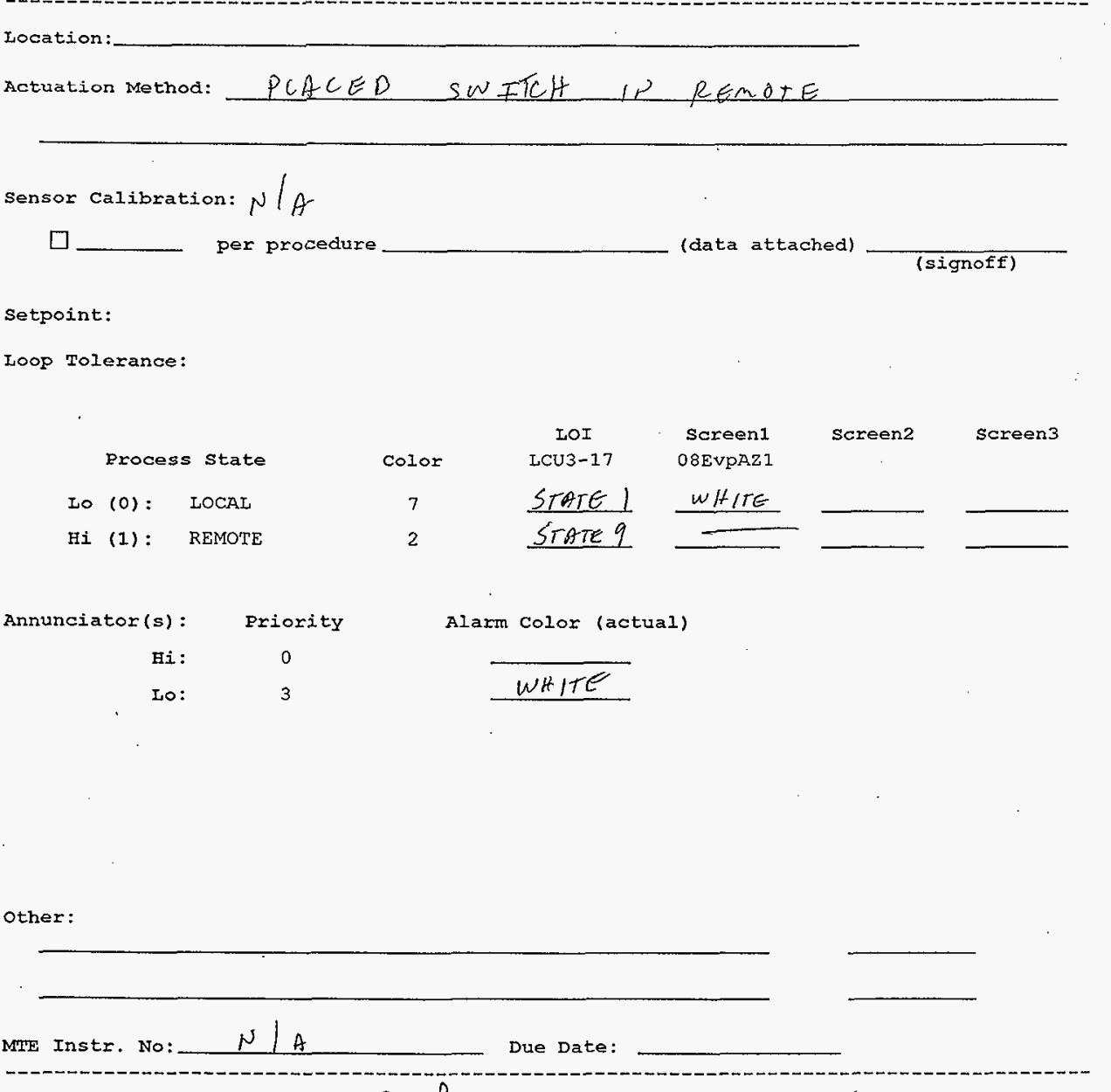

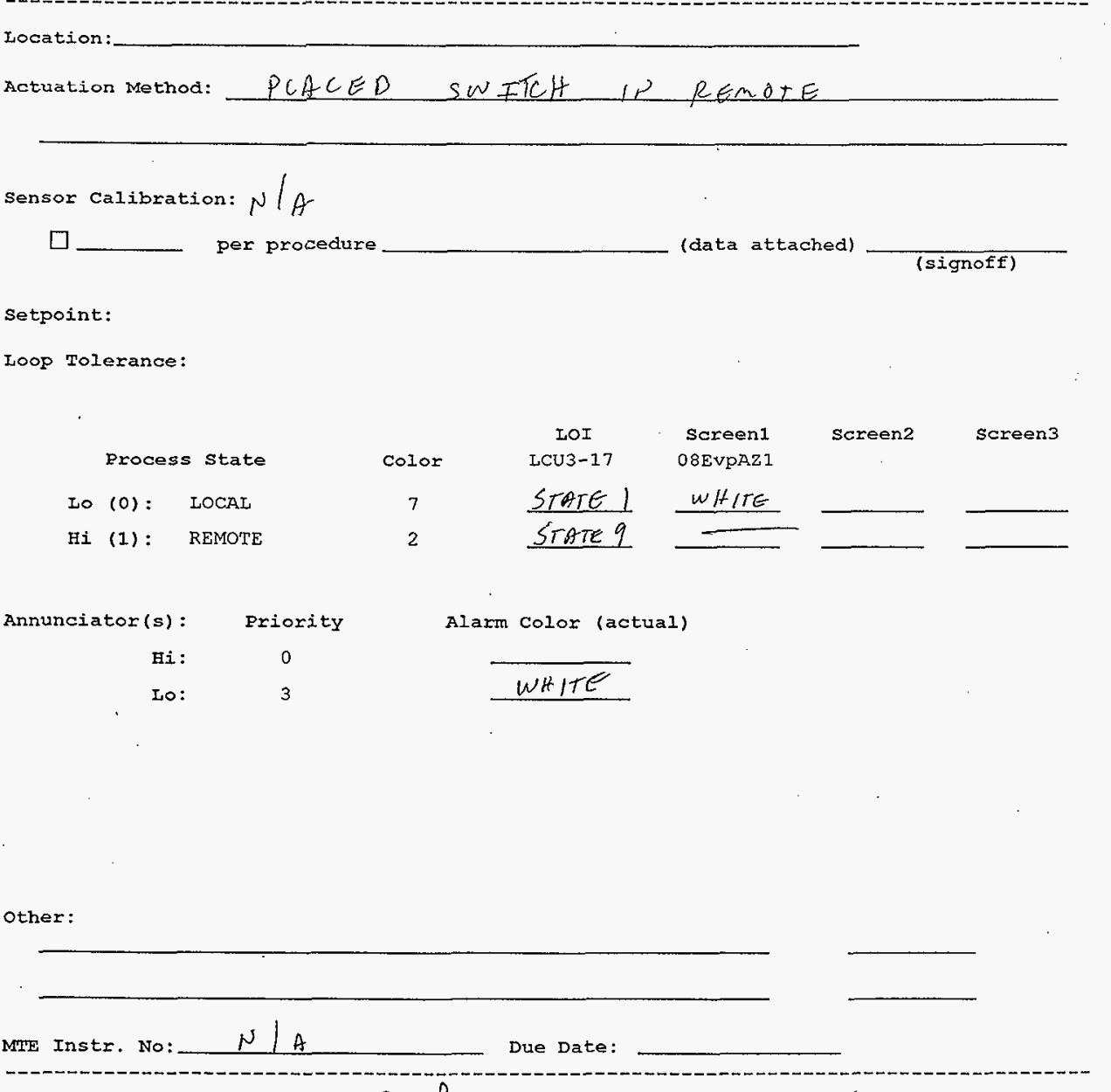

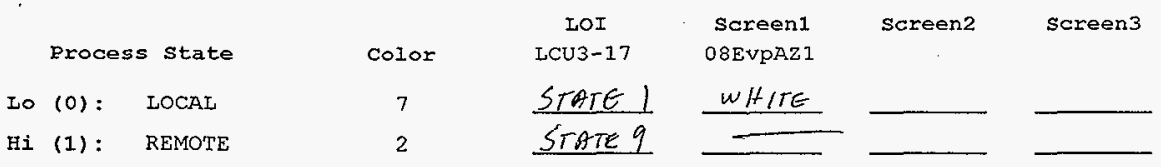

$\begin{array}{rcc}\text { Annunciator(s): } & \text { Priority } & \text { Alarm Color (actual) } \\ \text { Hi: } & 0 & \text { WH/TE } \\ \text { LO: } & 3 & \text { W }\end{array}$

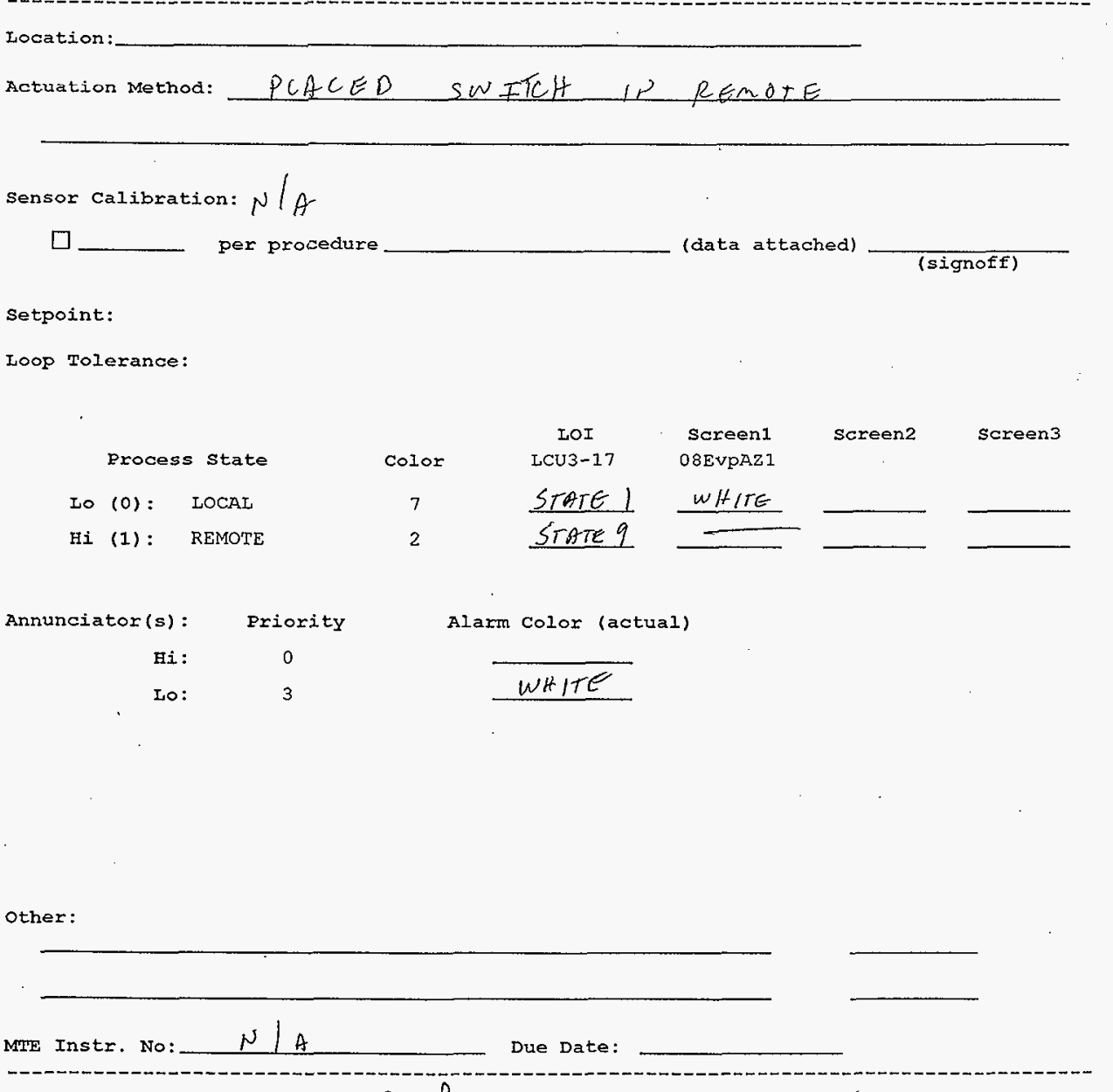

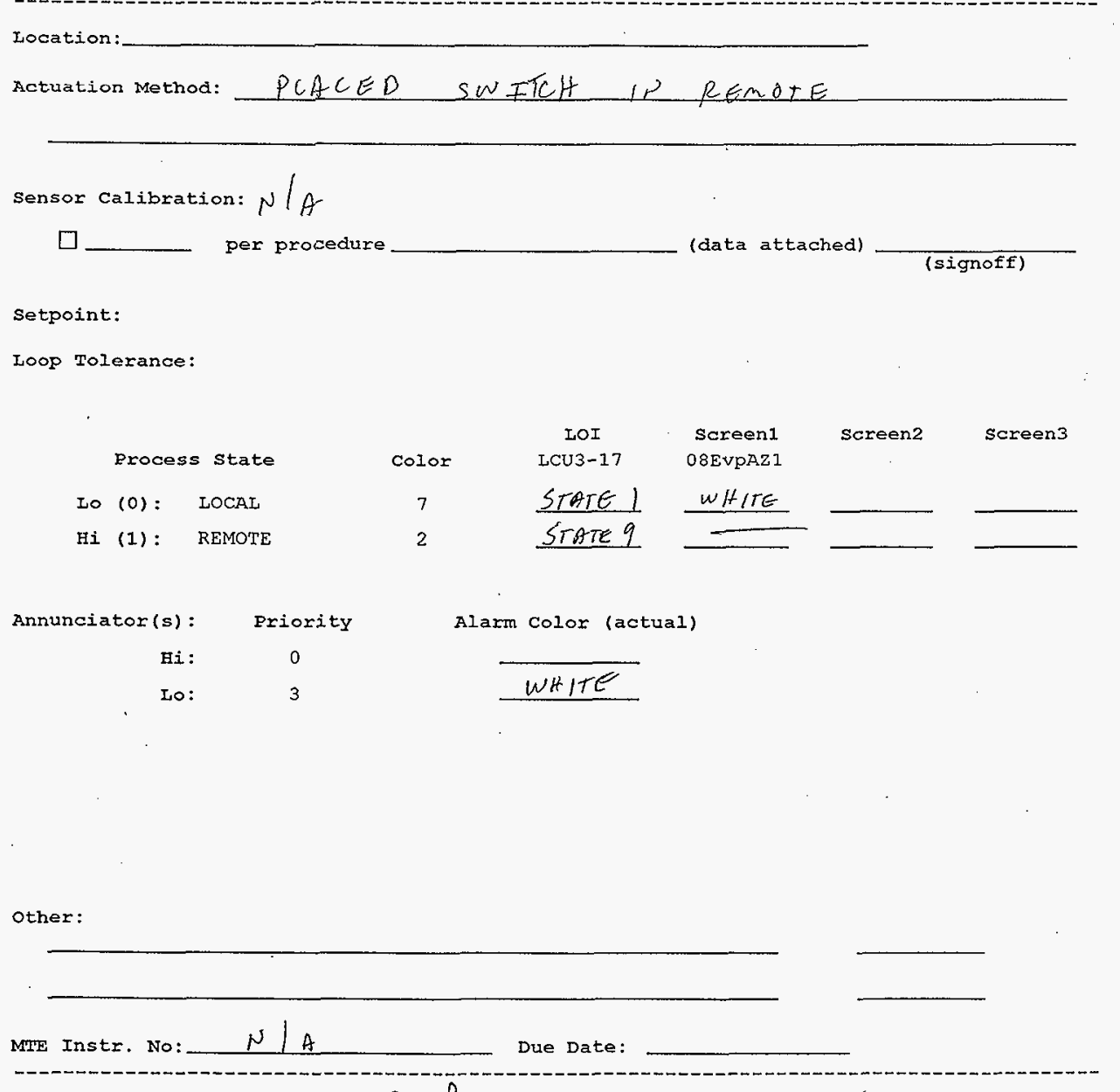

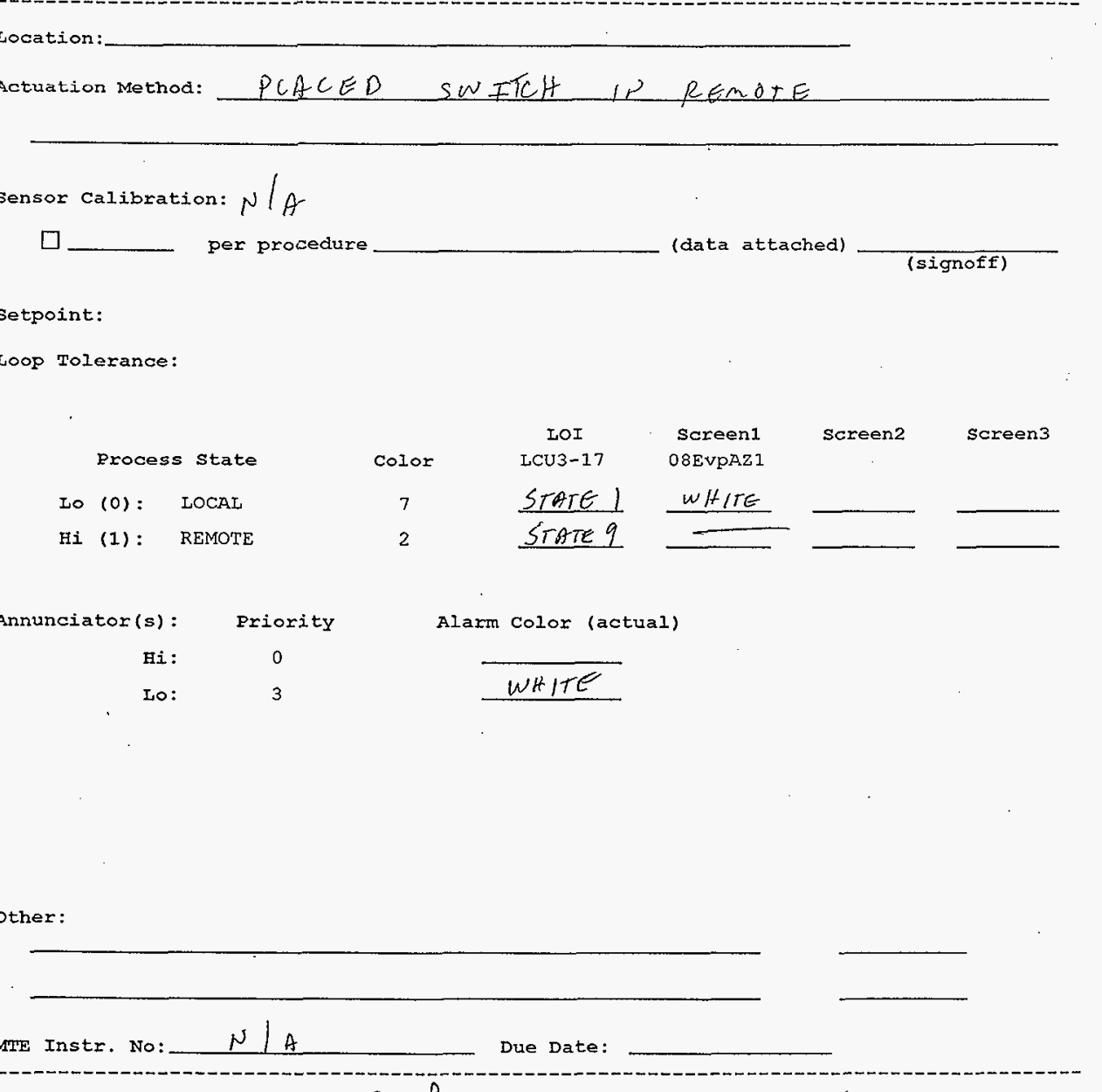

(signoff) Loop complete:_. 2. O. Date: $4 / 6 / 96$ 


\section{Tag: AZI__EWT_1A I/O Type: DO Description: EwTower On}

Critis: 54 chan No: 11 P\&ID:H-2-131069, SH.1 Iogic:

\section{Remarks :}

Location:

Actuation method: puce fistor fuses 5 seante sforter

Sensor Calibration:

$\square \ldots$ per procedure

$N / A$ (data attached)

Setpoint:

Loop Tolerance:

\begin{tabular}{|c|c|c|c|c|c|c|c|}
\hline & Process & state & Color & LOI & $\begin{array}{l}\text { Soreeñ } \\
\text { GROOP } \\
\text { RCNDR54-2 }\end{array}$ & Screen2 & Screen 3 \\
\hline Lo & $(0):$ & FALSE & 4 & & FALSE & 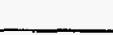 & \\
\hline $\mathrm{Hi}$ & (1): & TRUE & 7 & & & & \\
\hline
\end{tabular}

$\begin{array}{rcc}\text { Annunciator }(s): & \text { Priority } & \text { Alarm color (actual) } \\ \text { Hi: } & 0 & \\ \text { Lo: } & 0 & \end{array}$

Other:

MTE Instr. No:

Due Date:

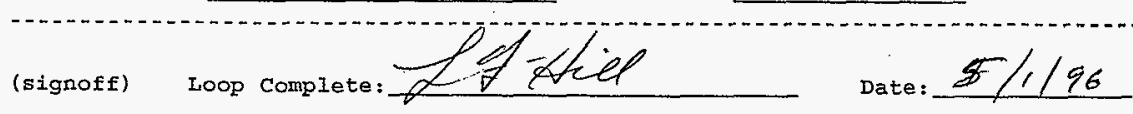


Tag: AZI___EWI_1B I/O Type: DO Description: EwTower , Off

CntIr: 54 Chan No: /2 P\&ID: H-2-131069, SH.l Logic:

Remarks:

Location :

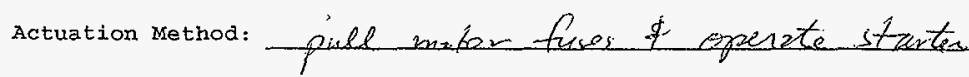

Sensor Calibration:

$\square \ldots$ per procedure

ULA (data attached)

(signoff)

Setpoint:

Loop Tolerance:

\begin{tabular}{|c|c|c|c|c|c|c|c|}
\hline & & & & IOI & Screen 1 & Screen2 & Screen 3 \\
\hline & Process & state & color & & & & \\
\hline Lo & $(0):$ & FALSE & 4 & & & & \\
\hline $\mathrm{HI}$ & $(1):$ & TRUE & 7 & 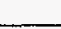 & 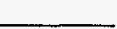 & ${ }_{2}$ & 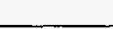 \\
\hline
\end{tabular}

$\begin{array}{rcc}\text { Annunciator }(s): & \text { Priority } & \text { Alarm Color (actual) } \\ \text { Hi: } & 0 & - \\ \text { Lo: } & 0 & \end{array}$

Other:

MTE Instr. No:

Due Date:

(signoff) soop complete: 2 thel

Date: $57 / 96$ 
Tag: AZ1. EWSP_18 I/O Type: DO

Description: EwSpPump Stop

Cotlr:

54 Chan No: 10

REID: $H-2-131069$, SH. 1 Logic:

Remarks :

Location:

actuation method: buel fures i openzte sterter

Sensor Calibration:

$\square \ldots$ per procedure

$N / A$ (data attached)

Setpoint :

Loop Tolerance:

Process State
LO (0): FALSE

$\begin{array}{rcc}\text { Annunciator(s) : } & \text { Priority } & \text { Alarm Color (actual) } \\ \text { Hi: } & 0 & \\ \text { LO: } & 0 & \end{array}$

Other:

MTE Instr. No: Due Date: (signofi) Ioop complete: 
Tag: $A Y 1$ EWP_IAB

CntIx:

49 Chan No: 6

Remarks :

Location:

Actuation Method: $w / T H \quad D C S$ SHOW $\sim 4$ START OPERATEN

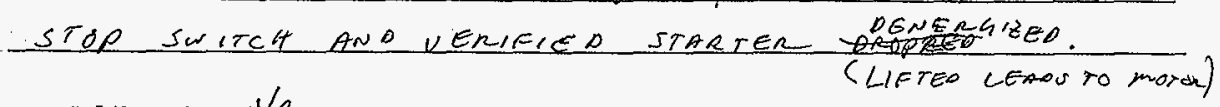
Sensor Calibration: $N / A$

$\square$ per procedure (data attached)

Setpoint:

Loop Tolerance:
Process State

Lo (0): FALSE

Hi (1): TRUE
IOI

color

\section{4}

7

$\begin{array}{rcc}\text { Annunciator (s) : } & \text { Priority } & \text { Alarm Color (actual) } \\ \text { Hi: } & 0 & \\ \text { LO: } & 0 & \end{array}$

\section{Other:}

NTE Instr. No:

Date: $4 / 15 / 46$ 
Tag: AY1_EWP_IAA I/O TYPe: DO Description: EwPump1A Start

Cntlr: 49 Chan No: 5 PEID: H-2.13106\%, SH.1 Logic:

Remarks:

Location:

Actuation Method: ACTJVATED SWITCH AT DCS AND

VERIFIEO CONTAETOR PULCEDIN. (LIETEO LENOS TO mator)

Sensor Calibration: $N / A$

$\square$ (data attached)

Setpoint:

Loop Tolerance:

\begin{tabular}{|c|c|c|c|c|c|c|c|}
\hline \multicolumn{3}{|c|}{ Pxocess state } & \multirow{2}{*}{$\begin{array}{c}\text { Color } \\
4\end{array}$} & \multirow[t]{2}{*}{ LOI } & \multirow[t]{2}{*}{ Sareenl } & \multirow[t]{2}{*}{ Screen 2} & \multirow[t]{2}{*}{ Screen 3} \\
\hline Lo & $(0):$ & FALSE & & & & & \\
\hline$H i$ & (1): & TRUE & 7 & & & & \\
\hline
\end{tabular}

Annunciator(s): Priority

Alarm Color (actual)

$\begin{array}{ll}\text { Hi: } & 0 \\ \text { LO: } & 0\end{array}$

other:

MTE Instr. No: Due Date:

(signoff) Loop complete: D.\&

Date: $4 / 15 / 46$ 
Tag: YS AZ2 EWSP_1 I/O TYPe: DI

Description: Az2EWSpyPumpsts

Cntlr:

53 Chan No:

21

P\&ID: $\mathrm{H}-2-131070$

Iogic:

Remarks:

Location:

Actuation method: pule fuses Ex pule is straver

\section{Sensor Calibration:}

$\square$ per procedure

N) I/A

(data attached)

(signoff)

Setpoint:

Loop Tolerance:

\begin{tabular}{|c|c|c|c|c|c|c|c|}
\hline & & & & LOI & screen 1 & Screen2 & screen 3 \\
\hline & Process & 3 state & Color & LCU3-21 & 09EvpAZ2 & 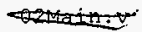 & Q2I0ALt \\
\hline Lo & (0): & OFE & 4 & sibta 0 & $\theta+s$ & & \\
\hline $\mathrm{Hi}$ & (1): & ON & 7 & stzet & biled & & \\
\hline
\end{tabular}

$\begin{array}{rcc}\text { Annunciator(s): } & \text { Priority } & \text { Alarm Color (actual) } \\ \text { Hi: } & 0 & \\ \text { LO: } & 0 & \end{array}$

Other:

MIE Instr. No:

Due Date:

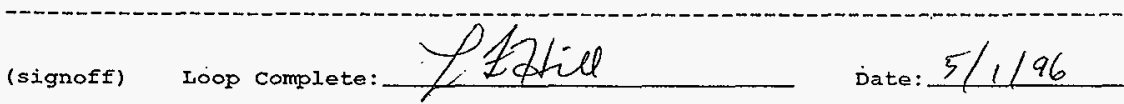


cntIr: 53

Chan No: 22

P\&ID: H-2-131070

Iogic:

Remarks :

Location:

Actuation Method: 1 iff motor lo ads of operta state

Sensor Calibration:

口_... per procedure U / A

(signoff)

Setpoint:

Loop Tolerance:

\begin{tabular}{|c|c|c|c|c|c|c|c|}
\hline & & & & IOI & screent & Screen2 & screen 3 \\
\hline & Process & s state & Color & LCU3-21 & 09EvpAZ2 & & \\
\hline Lo & $(0):$ & DISABLED & 4 & Stat 0 & ne gens & & \\
\hline Ez & (1): & ENABLED & 7 & strte 1 & Auto & & . \\
\hline
\end{tabular}

Annunciator(s): Priority Alarm color (actual)

Hi: $\quad 0$

\section{Other:}

MTE Instr. No:

Due Date:

(signoff) Loop complete:


Tag: YS_AZ2 _EWP_1A1 I/O Type: DI

Description: AZ2EWPmpA RemLoc

Cntir:

Chan No:

25

P\&ID: $\mathrm{H}-2-131070$

Iogic:

Remarks :

Location:

Actuation Method:

opperate losel switzl

Sensor Calibration:

$\square$. pex procedure

N)A (data attached)

Setpoint:

Loop Tolerance:

\begin{tabular}{|c|c|c|c|c|c|c|c|}
\hline & & & & LOI & Screenl & Screen 2 & Screen 3 \\
\hline & Process & 5 state & Color & $\mathrm{LCU} 3-22$ & 09EvpAz2 & & \\
\hline Lo & $(0):$ & LOCAL & 7 & STAR:1 & WHIFE & 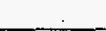 & \\
\hline $\mathrm{Hi}$ & (1): & REMOTE & 2 & STate? & & & \\
\hline
\end{tabular}

Annunciator (s): Priority

$\begin{array}{ll}\text { Hi: } & 0 \\ \text { Lo: } & 3\end{array}$

Alarm color (actual)

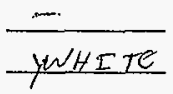

other:

MPE Instr. No:

Due Date:

(signoff) Loop complete: 


Tag: YS_AZ2_EWP_1BI I/O TYPe: DI Description: AZ2EWPmpB RemLOC

Cnt1r: 53 Chan No: 26 P\&ID: H-2-131070 Logic:

Remarks:

Location:

Actuation Method: repente locil suritel

Sensor Calibration:

口... per procedure

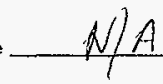
(data attached)

setpoint:

Loop Tolerance:

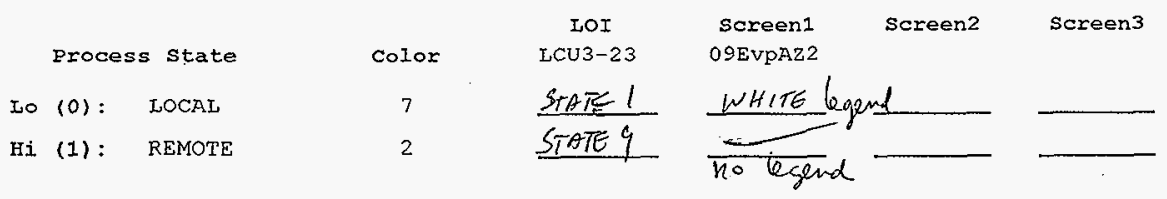

$\begin{array}{rcc}\text { Annunciator }(s): & \text { Priority } & \text { Alarm color (actual) } \\ \text { Hi: } & 0 & \text { WltLre } \\ \text { LO: } & 3 & \text { W }\end{array}$

Other:

MTE Instr. No:

Due Date: 
Tag: $A Z 2$ EWSP_1A I/O Type: DO

CntIr:

Remarks :

Location:

Actuation method: puef finsos stepenzte staver

Sensor Calibration:

$\square \ldots$ per procedure $N / 4$ (data attached)

Setpoint:

Loop Tolerance:

Process State
LO (0): Color
Hi (1): TRLSE

$\begin{array}{rcc}\text { Annunciator (s): } & \text { Priority } & \text { Alarn Color (actual) } \\ \text { Hi: } & 0 & \\ \text { Lo: } & 0 & \end{array}$

Other:

MTE Instx. No: Due Date:

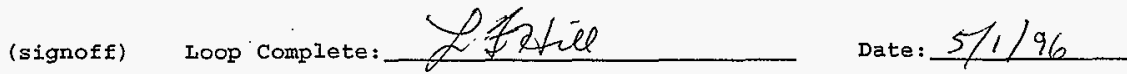


Tag : $\mathrm{AZ2}$ EWSP_1B I/O Type: DO

Description: EwSpPump Stop

CntIr:

54 Chan No: 25

P\&ID: $H-2-131070$, SH.I Logic:

Remarks :

Iocation:

Actuation Method: pulp fuses \& openzte starte

Sensor Calibration:

$\square$

per procedure

N/A

(data attached)

Setpoint :

Ioop Tolerance:

Process state

Io (0) : FALSE

Hi (1) : TRUE

Annunciator (s) :

Hi. :

Io:
LOI

color

4

7 screen1 Screen2

Screen3

Other:

Alarm Colox (actual)

0

o

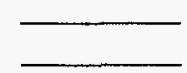


Tag: AZ2_EWT_IA I/O Type: DO

Description: EwTower on

Cntir: 54 Chan No: 26 2EID:H-2-131070, SH.1 Iogic:

Remarks :

Location:

Actuation Method: puel fues \& operzte sturte

Sensor Calibration:

per procedure $N / 4$ (data attached)

Setpoint:

Loop Tolerance:

$\begin{array}{lcccc} & & \text { LOI } & \text { Screen1 } & \text { Screen2 } \\ \text { Process State } & \text { Color } & & & \\ \mathrm{Hi}(\mathrm{I}): \text { FALSE } & 4 & & \end{array}$

$\begin{array}{rcc}\text { Annunciator (s) : } & \text { Priority } & \text { Alarm color (actuaI) } \\ \text { Hi: } & 0 & \\ \text { Lo: } & 0 & \end{array}$

other:

MTE Instr. No:

Due Date:

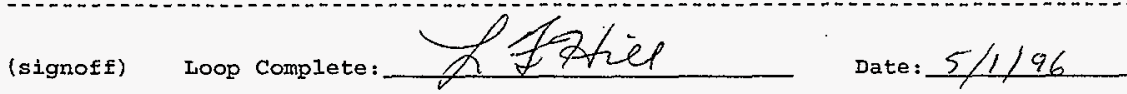


Tag: AZ2__EWT_1B IB I/ Type: DO

Description: EwTower

Off

Cntlr:

54 Chan No: 27

P\&ID: $H-2-131070,5 H .1$ Iogic:

Remarks :

Location:

Actuation Method:

- pues-Gases \& openzts starter

Sensor Calibration:

$\square$ - pex procedure

$N / A$ (data attached)

(signoff)

Setpoint:

Loop Tolerance:

Process state

Io (0): FAISE

Hi (1): TRUE

Annunciator (s) :

Fi:

Lo: priority

0

0
LOI

Colox

4

7
Screen1. Screen2

\section{Screen3}

Alarm Color (actual)
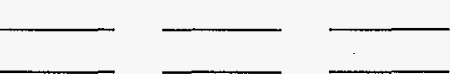

Other:

MTE Instr. No:

Due Date:

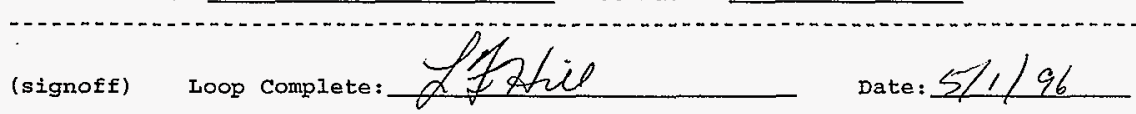


Tag: AZ2_EWP_1AA I/O TYPe: DO Description: EwPumplA Start

CntIr: 54 Chan No: 20 P\&ID:H-2-131070, sH.1 Logic:

Remarks :

Location:

Actuation method: Liff motor leads ft gyenats starten

Sensor Calibration:

$\square$ per procedure

1) $A$ (data attached)

(signoff)

Setpoint:

Loop Tolerance:

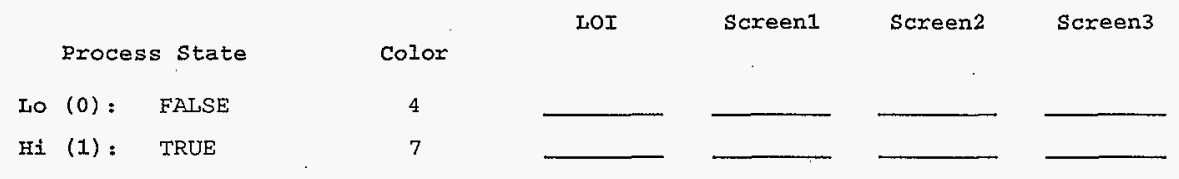

$\begin{array}{rcc}\text { Annunciator }(s): & \text { Priority } & \text { Alarm Color (actual) } \\ \text { Hi: } & 0 & \\ \text { Lo: } & 0 & \end{array}$

other:

MTE Instr. No:

Due Date:

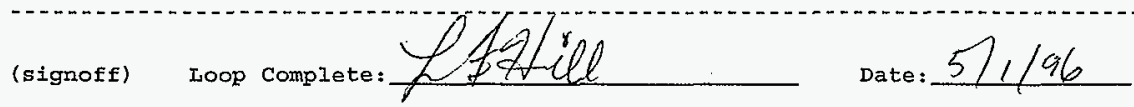


Tag: Az2 EWP_1AB

Cnt1r:

Remarks:

Location:

Actuation method: 1 iff motor 6 ads s prenzlestziter

Sensor Calibration:

$\square$. per procedure
N)A (data attached)

Setpoint :

Ioop Tolerance:

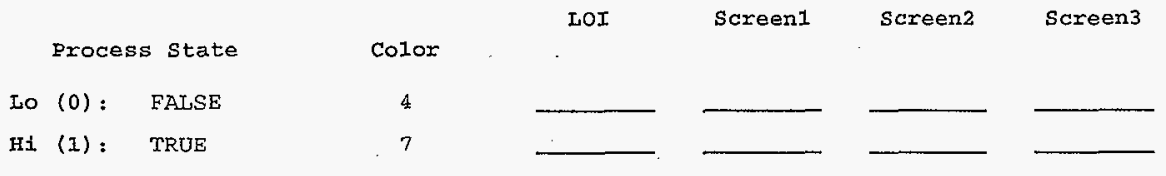

Hi: $\quad 0$

Other:

Alarm Color (actual)

Annunciator (s) : $\quad$ Priority

$$
\text { Alaxm Colox (actual) }
$$

MTE Instr. NO:

Due Date:

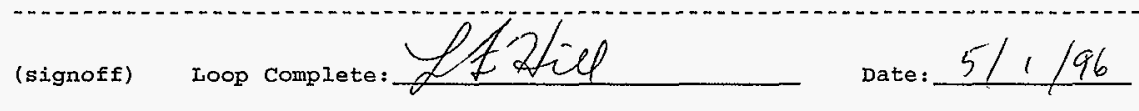


Tag: AZ2 EWP_IBA

CntIr:

Chan No: 22

PEID : H-Z-131070, SH.l Logie:

Remarks :

Location :

Actuation Method: ifi notor 6
per procedure $\frac{(\mathrm{A} /)}{4}$ eids \& zetrezte starea

Sensor Calibration:

$\square$ (data attached)

Setpoint:

LOOP Tolexance:

process State
LO (0): FAISE
Hi (I): TRUE

$\begin{array}{rcc}\text { Annunciator (s): } & \text { Priority } & \text { Alarm Color (actual) } \\ \text { Hi: } & 0 & \\ \text { LO: } & 0 & \end{array}$

Other:

MTE Instr. No: Due Date:

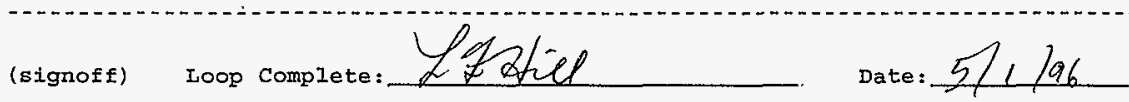


Tag: AZ2 EWP_IBB

Cnt1r:

Remarks :

Location:

Actuation Method: lif motor londs \& soenzte stavter

Sensor Calibration:

$\square$ per procedure $N / A$ (data attached)

Setpoint:

Loop Tolerance:

\begin{tabular}{|c|c|c|c|c|c|c|c|}
\hline & . & & & INOI & Scxeen1 & Screen2 & screen 3 \\
\hline & Process & State & Color & & & & \\
\hline Lo & $(0):$ & FAISE & 4 & & & $\therefore$ & \\
\hline Hi & (1): & TRUE & 7 & & & & \\
\hline
\end{tabular}

$\begin{array}{rcc}\text { Annunciator (s): } & \text { Priority } & \text { Alarm Color (actual) } \\ \text { Hi: } & 0 & \\ \text { IO: } & 0 & \end{array}$

Other:

MTE Instr. No: Due Date:

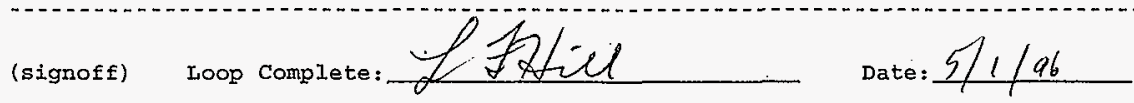


Tag: AZI_EWSP_1A I/O Type: DO Description: EwSpRump Start

Cntir: 54 Chan No: 9 P\&ID: H-Z-1310 69 , SH. I Logic:

Remarks :

Location:

Actuation Method: pull mator fuses F opente ctater

Sensor Calibration:

$\square$

pex procedure
$N / A$ (data attached)

Setpoint:

Loop Tolerance:

Process State

Io (0): FALSE

Hi (1) : TRUE

Annunciator (s) :

Hi:

LO:

\section{Priority}

0

0
IOI

color

4

7
Screen1 Screen2 Screen3

Alarm Color (actual)

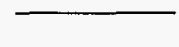

othex:

MTE Instr. No: Due Date: (signoff) Loop complete: Date: $51 / 96$ 
Tag: AZ1_EWP_IAA I/O Type: DO Description: EwPumplA Start

CntIr: 54 Chan No: 5 R\&ID: H-Z-131076, SH. I IOgIC:

Remarks :

Location:

Actuation Method:

prelin Strotion

witerats iffed

Sensor Calibration:

$\square$

per procedure

L) $/ A$ (data attached)

Setpoint:

Loop Tolerance:

Process state

Lo (0): FALSE

Hi (1): TRUE
LOI

Color

4

7
Screen1 Scxeen2

screen 3

$\begin{array}{rcc}\text { Annunciator (s) : } & \text { Priority } & \text { Alarm Color (actual) } \\ \text { Hi: } & 0 & \\ \text { LO: } & 0 & \end{array}$

Other:

MTE Instr. No: Due Date:

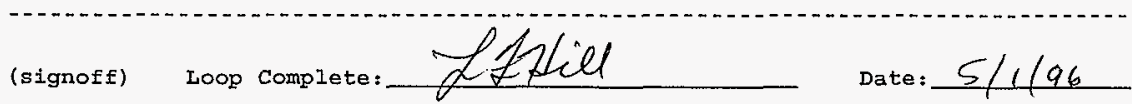


Tag: AZ1____ EWP_1AB I/O Type: DO Description: EwPump1A Stop

Cntir:

54 Chan No: 6

R\&ID: $H-2-131076,5 H \cdot 1$ Logic:

Remarks :

Location:

Actuation method: lift wotor loads \& peerate starla

Sensor Calibration:

$\square$

per procedure
$N A$ (data attached)

Setpoint:

Loop Tolerance:

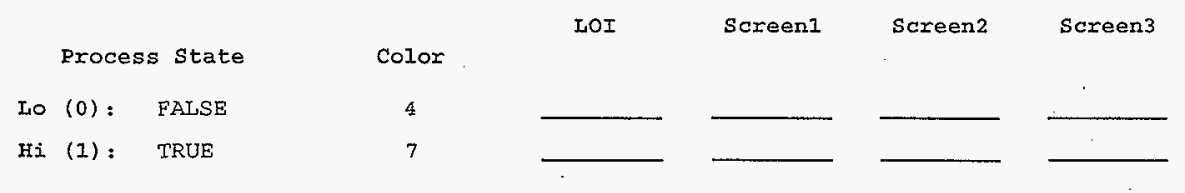

$\begin{array}{rcc}\text { Annunciator }(\mathrm{s}): & \text { Priority } & \text { Alarm Color (actual) } \\ \text { Hi: } & 0 & \\ \text { IO: } & 0 & \end{array}$

Other:

MTE Instr: No:

Due Date:

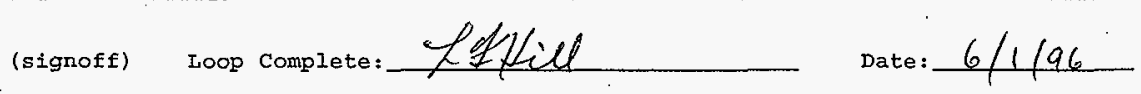


Tag: AZ1_EWP_1BA I/O Type: DO

Description: EwRump1B Start

Cntlr:

54. Chan No: 7

P\&ID: $H-2-131076,5 H .1$ rogic:

Remarks :

Location:

Actuation Method: Iift motor.6eds f capete cteren

Sensor Calibration:

$\square \ldots$ pex procedure.

NA

(data attached)

(signoff)

Setpoint:

Loop Tolerance:

Process State
LO $(0)$ : Color

$\begin{array}{rcc}\text { Annunciator(s): } & \text { Priority } & \text { Alarm Color (actuai) } \\ \text { Hi: } & 0 & \\ \text { LO: } & 0 & \end{array}$

other:

MTE Instr. No:

Due Date:

(signoff) Loop complete: ffEfiel Date: $5 / 1 / 96$ 
Tag: AZ1__ EWP_1BB I/O Type: DO

Description: EwPumplB Stop

CntIr: 54 Chan No: 8 R\&ID:H-2-131076, sH.1 Logic:

Remaxks :

Iocation:

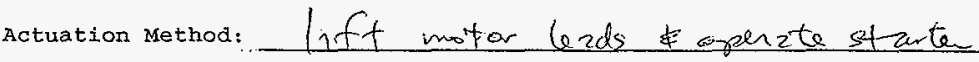

Sensor Calibration:

a _ per procedure

$N / A$

(data attached)

(signoff)

Setpoint:

Loop Tolerance:

\begin{tabular}{|c|c|c|c|c|c|c|c|}
\hline & Process & s state & Color & - LOI & Screenl & Screen2 & Screen 3 \\
\hline Lo & $(0):$ & FALSE & 4 & $\ldots$ & & & \\
\hline $\mathbf{H i}$ & (1): & TRUE & 7 & $\ldots$ & 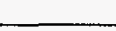 & 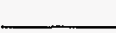 & $\ldots$ \\
\hline
\end{tabular}

$\begin{array}{rcc}\text { Annunciator (s) : } & \text { Priority } & \text { Alarm Color (actual) } \\ \text { Hi: } & 0 & \\ \text { Lo: } & 0 & \end{array}$

other:

MTE Instr. No:

Due Date:

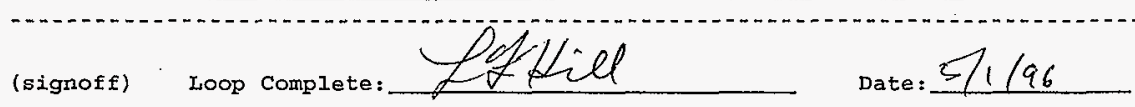


Tag: YS_AY 1_EWT_1A1 I/O TYpe: DI

Description: AY1EWTwrFansts

Cntir: 47

Chan No: 24

P\&ID: $\mathrm{H}-2-131067$

Logic:

Remarks :

Location:

Actuation Method: pull fuses $\$$ rpente stavien

Sensox Calibration:

$\square$ per procedure

$1 / A$ (data attached)

Setpoint:

Loop Tolexance:

\begin{tabular}{|c|c|c|c|c|c|c|c|}
\hline & & & & LOI & Screen1 & Screen2 & Screen 3 \\
\hline & Process & s state & Color & $\operatorname{LCU} 4-15$ & 0 6 EvPAY 1 & 02Main. $\mathrm{V}$ & 03LeakDt \\
\hline & $(0):$ & OFE & 4 & 0 & & & \\
\hline $\mathrm{Hi}$ & (1): & ON & 7 & & +716 & tillo & \\
\hline
\end{tabular}

$\begin{array}{rcc}\text { Anmunciator(s): } & \text { Prioxity } & \text { Alarm Color (actual) } \\ \text { Hi: } & 0 & \\ \text { Lo: } & 0 & \end{array}$

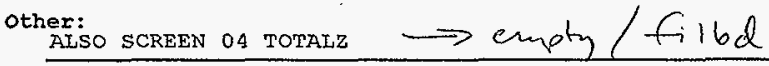

MIE Instr. No:

Due Date:

(signoff) Loop complete: 
Tag: YS_AY2_EWT_1AI I/O TYpe: DI Description: AY2EWTwrEansts

Cntlr: 48 Chan No: 24

P\&ID : H-2-131068

Logic:

Remaxks :

Location:

Actuation Method: puel fuses \& opente statea

sensor Calibration:

口 per procedure $\cup / 2 A$ (data attached)

Setpoint:

Loop Tolerance:

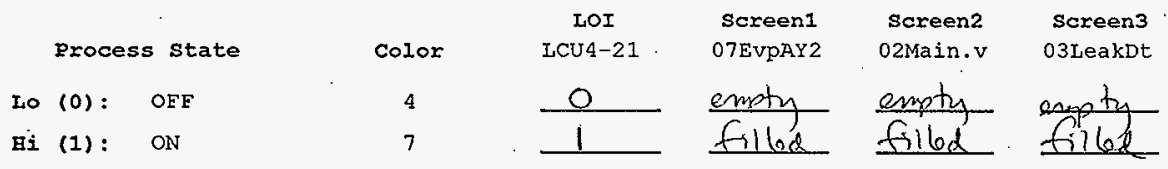

$\begin{array}{rcc}\text { Annunciator(s): } & \text { Priority } & \text { Alarm Color (actual) } \\ \text { Hi: } & 0 & \\ \text { Lo: } & 0 & \end{array}$

Other:
AISO SCREEN 04 Totalz empty/-Priled

MTE Instr. No:

Due Date: 
Tag: YS_AZ1_EWT_1A1 I/O TYPe: DI

Description: Az1EWTwrFansts

CntIr: 52

Chan No: 24

P\&ID: $\mathrm{H}-2-131069$

Logic:

Remarks :

Location:

Actuation Method: 1 ift motor leses \& peturta staten

Sensor Calibration:

$\square$

per procedure
$N / A$

(data attached)

setpoint:

Ioop Tolerance:

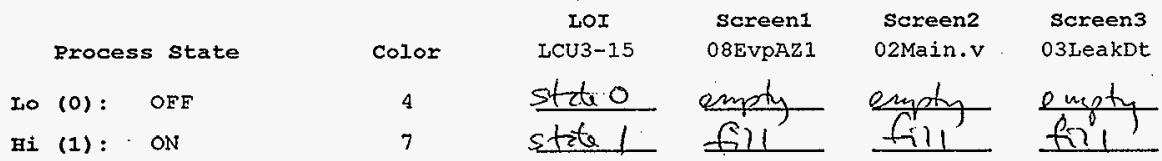

$\begin{array}{rc}\text { Annunciator(s): } & \text { Priority } \\ \text { Ei : } & 0 \\ \text { Lo: } & 0\end{array}$

Alarm color (actual)

other:

ALSO SREEN O4 TOTALz. $\rightarrow$ empty $/$ fil

MPE Instr. No:

Due Date:

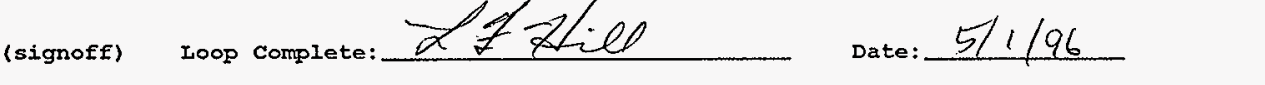


Tag: IS_AZ2_EWT_IAI I/O Type: DI Description: Az2EWTwrFansts

Cntlx: 53

Chan No: 24

P\&ID: $H-2-131070$

Iogic:

\section{Remaxks :}

Iocation:

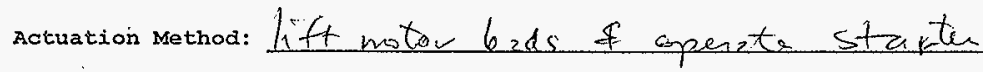

Sensor calibration:

$\square \longrightarrow$ per procedure $\frac{N / A}{T}$ (data attached)

(signoff)

Setpoint:

Loop Tolerance:

\begin{tabular}{|c|c|c|c|c|c|c|c|}
\hline & & & & IOI & Screen1 & Screen2 & Screen 3 \\
\hline & Process & state & Colox & LCU3-21 & O9EvpAZ2 & 02Main, $\mathrm{V}$ & 03 LeakDt \\
\hline Io & $(0):$ & OFF & 4 & stzes 0 & & & chmot \\
\hline $\mathrm{Hi}$ & (1): & ON & 7 & stat 1 & & & \\
\hline
\end{tabular}

$\begin{array}{rcc}\text { Annunciator (s): } & \text { Priority } & \text { Alarm Color (actual) } \\ \text { Hi: } & 0 & \\ \text { Lo: } & 0 & \end{array}$

ALSO SCREEN 04 TOTALZ $\rightarrow$ emoth $\rightarrow$ f lo le

MIE Instr. No:

Due Date:

(signoff) Loop complete: 
Tag: PI-AY101EWP-1A1

P\&ID : H-2-131067, SH 1

IOOP :

Manufacturer: MaDariel Contruls Mode1 No. : Safety Gaug a Serial No: $2 E-000-682$ MTE Instr. No: $817-35-40-015$ Due Date: $3-19-97$

Input Range 0-15

Tolexance $3 \%$

Units: PSI

Input : Output

$0 \quad 0$

7.5 PSF 7.5

15 PST 15

-10 in $H g-10$
-20 in $\mathrm{Hg}-20$

\begin{tabular}{ccccc} 
Lo: Hi: & As Found & In/Out As Left \\
-.45 .45 & 0.4 & IN & 0.4 \\
\hline 7.05 & 7.95 & 7.9 & IN & 7.9 \\
\hdashline-14.55 & 15.45 & 15.3 & IN & 15.3 \\
\hline-9.1 & -10.9 & -9.3 & IN & -9.3 \\
\hline-19.1 & -20.9 & -20.0 & IN & -20.0 \\
\hline
\end{tabular}

(signoff)Calibrated By: Fitzsimindns

Date: $2 \cdot 14 \cdot 97$

Location: AY10 1. Cooling Water tower (EwP-10-Pups-Swetion)

Comments Compound Gauge 
Tag: PI-AY101EWP-1A2

P\&ID: H-2-131.067, SH 1

LOOP :

Manufacturer: McDaniel Cantrols Model No.: Safety Gauge. Serial No: $2 \bar{E}-000-683$ MTE Instr. No: $817-35-40-013$ Due Date: $5 \cdot 30-97$

Input Range $0-60$

Units: PSI

Input Output

0

30

30

60

60

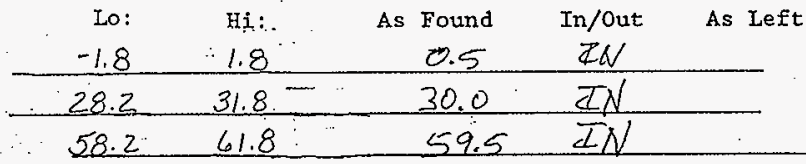

(signoff)Galibrated By: Fitzsimmons

Date: $2 \cdot 14-97$

Location: Cooling water tower (101Ay) (Ewe-1A-Pump Discharge)

Comments 
Tag: PI-AY101EWP-1BI

P\&ID: H-2-131067, SH 1

LOOP :

Manufacturer: MrDanid Controls Model no. : Safety Gange serial No:2R:000-684 MTE Instx. No: $817-35-40 \cdot 015$

Due Date: $3 \cdot 19 \cdot 97$

Input Range 0-15

Tolexance $3 \%$

Units : PSI

\begin{tabular}{cccccccc} 
Input & Output & Lo: & Hi: & As Found & In/Out & As Left \\
0 & 0 & -.45 & .45 & 0 & IN & 0. \\
7.5 PSI & 7.5 & 75 & 7.05 & 7.45 & 7.4 & IN & 7.4 \\
15. PSI & 15 & 14.55 & 15.45 & 14.8 & IN & 14.8 \\
$-10 I N H g$ & -10 & -9.1 & -10.9 & -9.6 & IN & -9.6 \\
-20 IN Hg -20 & -19.1 & -20.9 & -19.9 & IN & -19.9 \\
\hline
\end{tabular}

(signoff)calibrated By: Fitzsimmons

Date: $2 \cdot 14 \cdot 97$

Location: AY 101 Cooling water tower (EwP-1B Pump Suction. $)$

comments Compound Gauge 
Tag: PI-AY101EWP-IB2

P\&ID: $\mathrm{H}-2-131067$, SH 1

LOOP :

Manufacturer: MiDaniel Controls Model No.: Safety Gauge Serial No:2E-000-685. MTE Instr. No: $817-35-40-013$ Due Date: $5-30-97$.

Input Range $0-60$

Tolexance $3 \%$

Units: PSI

Input output

00

$30 \quad 30$

60

60

\begin{tabular}{ccccc} 
Lo: & Hi: & As Found & In/out & As Left \\
-1.8 & 1.8 & 0 & IN & 0 \\
\hline 28.2 & 31.8 & 30 & IN & 30 \\
\hline 58.2 & 61.8 & 59.8 & IN & 59.8
\end{tabular}

(signoff)Galibrated By: Fitzsimmons

Date: $2 \cdot 14 \cdot 97$

Location: Ay 101 Cooling Water Tower (Eisp-1B - Pump Discharge)

Comments 
Tag: PI-AYLOLWS-1

P\&ID : $\mathrm{H}-2-131067$, SH 1

LOOP:

Manufacturer: Ashcroft Mode1 No. : Duralife Seria1 No: $2 E \cdot 000-681$ MTE Instx. No: $817-35-40.011$ Due Date: $5-22-97$

\begin{tabular}{|c|c|c|c|c|c|c|}
\hline \multirow{2}{*}{\multicolumn{2}{|c|}{$\begin{array}{l}\text { Input Range } 0-60 \\
\text { Units: PSI }\end{array}$}} & \multirow{2}{*}{\multicolumn{3}{|c|}{ Tolerance $5 \%$}} & \multirow{2}{*}{\multicolumn{2}{|c|}{$\cdot$}} \\
\hline & & & & & & \\
\hline Input & \multirow{2}{*}{$0^{\text {output }}$} & Lo: & $\mathrm{Hi:}$ & As Found & In/Out & As Left \\
\hline 0 & & -3 & +3 & e) & $I N$ & 0 \\
\hline 15 & 15 & 12 & 18 & 14.5 & IN & 14.5 \\
\hline 30 & 30 & 27 & 33 & 29.5 & $Z N$ & 29.5 \\
\hline 45 & 45 & 42 & 48 & 44.5 & $\mathbb{Z N}$ & 44.5 \\
\hline 60 & 60 & 57 & 63 & 59.5 & $\geq N$ & 59.5 \\
\hline
\end{tabular}

(signoff)calibrated By: Fitzsimmons Date: $-3-6-97$

Location:

Comments 
Tag：PI-AY101WS-2

P\&ID : H-2-131067, SH 1

LOOP:

Manufacturer: Ashcreft Model No.: Puralife

Serial No: 2E-000-680 MTE Instr. No: $817-35-40-011$

Due Date: $5-22-97$

Input Range $0-60$

Units : PSI

Input

0

15

30

45

60

60

\begin{tabular}{ccccc} 
Lo: & Hi: & As Found & In/out & As Left \\
-3 & +3 & 4 & out & 0 \\
\hline 12 & 18 & 18 & IN & 15 \\
\hline 27 & 33 & 33 & $I N$ & 30 \\
\hline 42 & 48 & 48 & $I N$ & 44.5 \\
\hline 57 & 63 & 63 & $\nexists N /$ & 60 \\
\hline
\end{tabular}

(signoff)Calibrated By: Fitzsimmons

Date: $3-6-97$

Location:

Comments 
$2 / 14 / 97$

W-030 CALIBRATION DATA SHEET

HNF-SD-W030-TD-003,

REV. 0, PAGE 286

Tag: PI -AY102EWP-1A1

P\&ID: H-2-131068, SH 1

LOOP :

Manufacturer: McDaniel Controls Model No.: Safety Gauge Serial No:2E-000.677

MTE Instr. No:

$817 \cdot 35 \cdot 40=015$

Due Date: $3-19 \cdot 97$

Input Range 0-15

Units: PSI

$\begin{array}{cc}\text { Input } & \text { Output } \\ 0 & 0 \\ 7.5 \text { PSI } & 7.5 \\ 15 \text { PSI } & 15 \\ -10 \mathrm{inHg} & -10 \\ -20 \mathrm{in} \mathrm{Hg} & -20\end{array}$
Tolerance $3 \%$

\begin{tabular}{ccccc} 
Lo: & Hi: & As Found & In/ out & As Left \\
-.45 & .45 & 0 & IN & 0 \\
\hline 7.05 & 7.95 & 7.4 & IN & 7.4 \\
\hline 14.55 & 15.45 & 15.2 & IN & 15.2 \\
\hline-9.1 & -10.9 & -9.2 & IN & -9.2 \\
\hline-19.1 & 20.9 & -19.0 & IN & -19.0 \\
\hline
\end{tabular}

(signoff) Calibrated By: Fitzsimmons

Date: $2 \cdot 14 \cdot 97$

Location: By 102 Cooling Water Pad (EwP-1R-Pump Suction)

Comments Compound Gauge 
Tag: PI-AY102EWP-IA2

P\&ID: H-2-131068, SH 1 LOOP:

Manufacturer: McDaniel Controls Model No.: Safety Gauge serial No:2E-000.674 MTE Instx. No: $817 \cdot 35-40-013$ Due Date: $5 \cdot 30 \cdot 97$

Input Range $0-60$

Units: PSI

Input

0

30

60 output

0

30

60
Tolerance $3 \%$

\begin{tabular}{ccccc} 
Lo: & Hi: & As Found & In/out & As Left \\
-1.8 & 1.8 & 0 & IN & 0 \\
\hline 28.2 & 31.8 & 29.9 & IN & 29.9 \\
\hline 58.2 & 61.8 & 60.0 & IN & 60.0 \\
\hline
\end{tabular}

(signoff)Calibrated By: $\overline{F i t z s i m m o n s ~}$

Date: $2 \cdot 14 \cdot 97$

Location: AY 102 Cooling water Tower (EwP-1A-Pump Discharge)

Comments 
Tag: PI-AY102EWP-1BI

P\&ID : H-2-131068, SH 1

LOOP:

Manufacturer: MaDaniel Controls Model No. : Safety. Guuge serial No: $22-000-676$ MTE Instx. No: $817-35-40-015$ Due Date: $3-19.97$

Input Range 0-15

Tolerance $3 \%$

Units: PSI

Input output

OPSI 0

7.5 PSF 7.5

15PSI 15

-10 INHy -10

$-20 \mathrm{IN} \mathrm{Hg}-20$

\begin{tabular}{ccccc} 
Lo: & Hi: & As Found & In/Out & As Left \\
-.45 & .45 & 0 & IN & 0 \\
\hline 7.05 & 7.95 & 7.1 & IN & 7.1 \\
\hline 14.55 & 15.45 & 14.8 & IN & 14.8 \\
\hline-9.1 & -10.9 & -10.0 & IN & -10.0 \\
\hline-19.1 & -20.9 & -19.9 & IN & -19.9 \\
\hline
\end{tabular}

(signoffXalibrated By: Fitzsimmons

Date: $2 \cdot 14 \cdot 97$

Location: Ay 102 Cooling Water Tower (Ewe-1B-Pump Suction) 
Manufactuxer: McDaniel Controlsmodel No.: Safety Gauge serial No: 2E.000.675 MTE Instr. No: $817-35 \cdot 40-013$ Due Date: $5-30-97$

Input Range $0-60$

Units : PSI

Input output

00

$30 \quad 30$

6060
Tolerance $3 \%$

\begin{tabular}{|c|c|c|c|c|}
\hline $\begin{array}{r}\text { Lo: } \\
-1.8 \\
\end{array}$ & $\begin{array}{l}\mathrm{Hi}: \\
1.8 \\
\end{array}$ & $\begin{array}{c}\text { As Found } \\
0\end{array}$ & $\begin{array}{c}\text { In/out } \\
\text { IN }\end{array}$ & $\begin{array}{c}\text { As Left } \\
0\end{array}$ \\
\hline 28.2 & 31.8 & 29.8 & $I N$ & 29.8 \\
\hline 58.2 & 61.8 & 59.8 & $I N$ & 59.8 \\
\hline
\end{tabular}

(signoff)Galibrated By: Fitzsimmons

Date: $2-|4-9\rangle$

Location: Ay 102 Cooling Water Tower (EwP-1B-Pump Discharge) 
HNF-SD-W030-TD-003, REV. O, PAGE 290

Tag: PI-AY102WS -1

P\&ID : H-2-131068, SH 1

LOOP:

Manufacturex: Ashcroft

Model No.: Duralife

Serial No: $2 E-000-679$

MTE Instr. No: $817-35-40.011$

Due Date: $5-22.97$

Input Range 0-60

Tolerance $5 \%$

Units : PSI

$\begin{array}{cc}\text { Input } & \text { Output } \\ 0 & 0 \\ 15 & 15 \\ 30 & 30 \\ 45 & 45 \\ 60 & 60\end{array}$

\begin{tabular}{ccccc} 
Lo: & $\begin{array}{c}\text { Hi: } \\
-3\end{array}$ & As Found & In/Out & as Left \\
\hline 3 & 18 & 0 IN & IN & 2 \\
\hline 12 & 33 & 15 & $I N$ & 15 \\
\hline 27 & 30 & $I N$ & 30 \\
\hline 42 & 48 & 45 & $I N$ & 45 \\
\hline 57 & 63 & 60 & $I N$ & 60 \\
\hline
\end{tabular}

(signoffjalibrated By: Fifzsiminwes

Date: $3-6-97$

Location:

Comments 
Manufacturer: Ashcroft Mode1 No.: Duralife Serial No:2E-000.678 MTE Instr. No: $817-35-40-011$ Due Date: $5 \cdot 22-97$

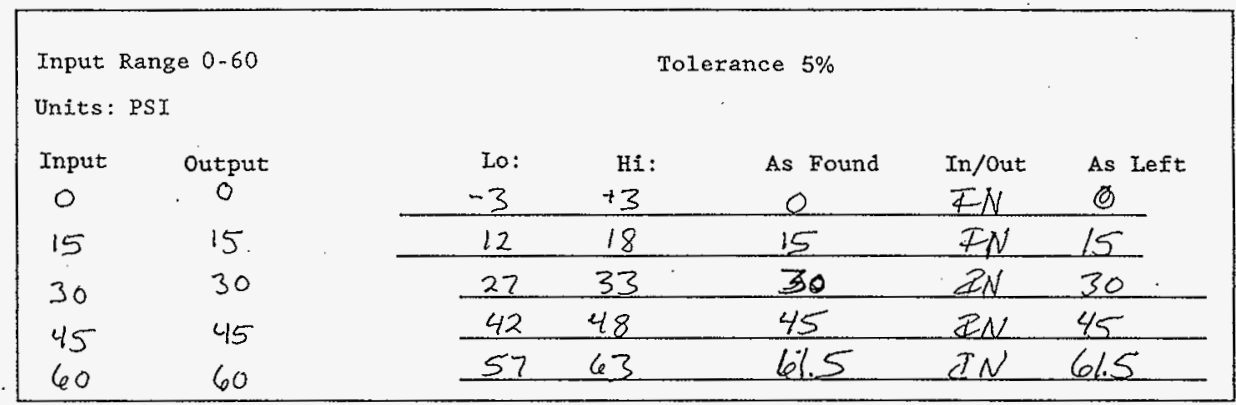

(signoff)Calibrated By: Fitzsimmons Date: $-3-6-97$

Location: 
$6 / 6 / 96$

H-030 CALIBRATION DATA SEEL

$$
\begin{aligned}
& \text { HNF-SD-W030-TD-003, } \\
& \text { REV. 0, PAGE } 292
\end{aligned}
$$

Tag: PI.AZ|0|EंWP-|A| P\&ID:

Lune.

Manufacturer: McDaniel Controls Model No.: Safety Gauge serial no: 2E 000b loo

Mere Instr. No: $817 \cdot 35 \cdot 40 \cdot 030$

Due Date: $7 \cdot 31 \cdot 97$

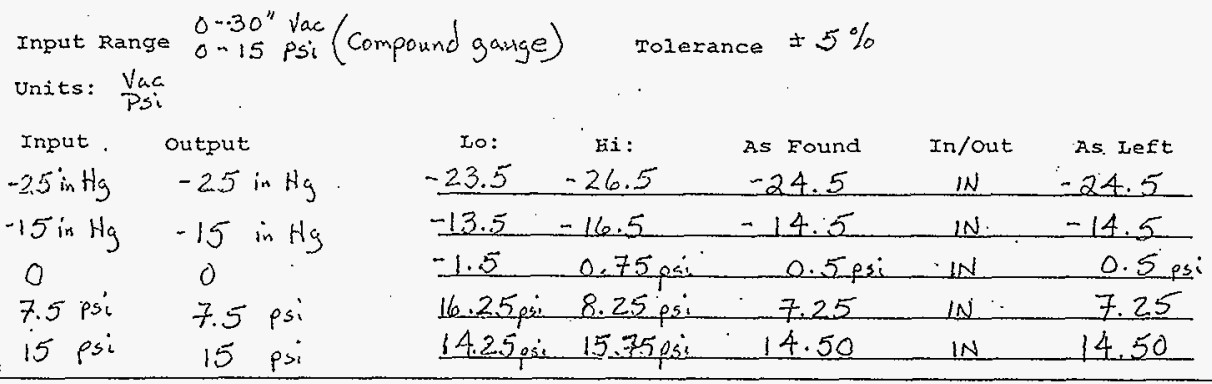

(signoff) Calibrated BY:

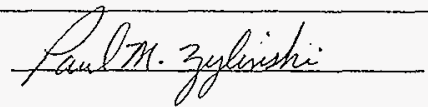

Date: $2: 5 \cdot 97$

Location: $101 A z$ Cooling water tower (Ewp-lA pump suction)

comments Gruporend gauge. 
rag: PI. AZ101EWP-IAZ

PEXD:

HNF-SD-W030-TD-003, REV. 0, PAGE 293

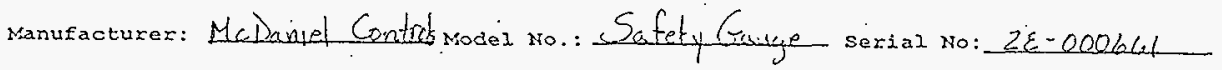
MTE Instr. No: $-817 \cdot 35 \cdot 40 \cdot 011$ Due Date: $5 \cdot 22 \cdot 97$

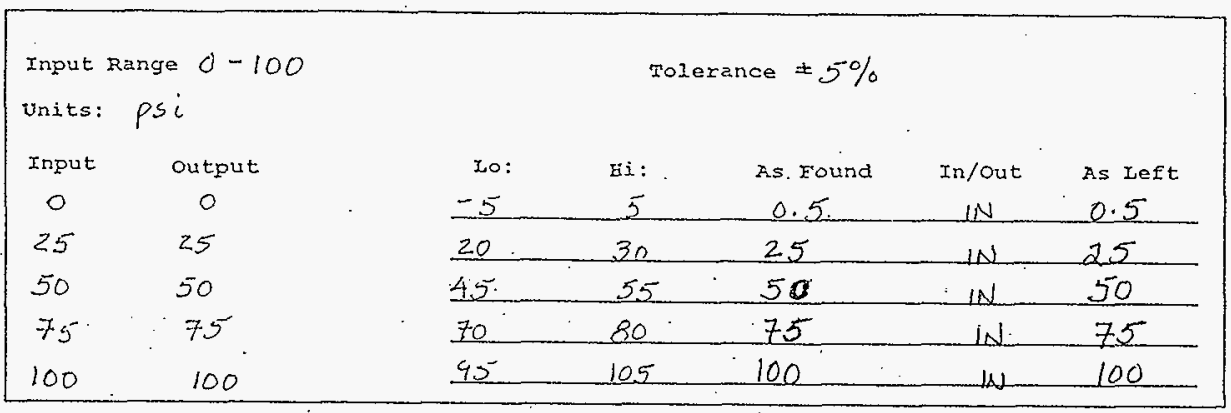

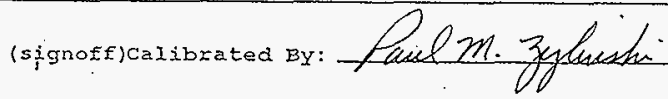

Date: $2 \cdot 5 \cdot 97$

Location: Iol Az Conling water timer (Ewp A Pump Discharge)

Comments 


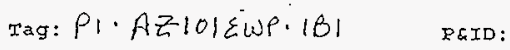

HNF-SD-W030-TD-003,

REV. 0, PAGE 294

I $\infty$ P:

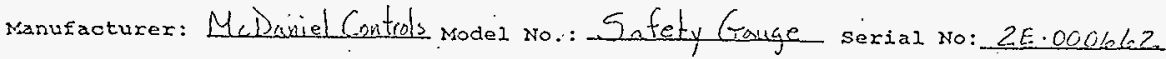

Me Instr. No: $-817: 35 \cdot 40 \cdot 030$

Due Date: $7 \cdot 31 \cdot 97$

Input Range $0-30^{\circ} \mathrm{Hg} \mathrm{VaC}$

Tolerance $\pm 5 \%$

Units: in $\mathrm{Hg}_{\mathrm{VaC}} \mathrm{O}^{-} \mathrm{Psi}$ Psi.

Input output

-25 in $H_{3}-25 \mathrm{in} \mathrm{H}_{5}$

-15 in $\mathrm{H}_{\mathrm{j}}-15 \mathrm{in} / \mathrm{lg}$

7.5 .0

7.5 ps: $7.5 \mathrm{psi}$

Lo:

Rs. Found

snout

As Left

$-23.5 \quad-26.5 \quad-24.5$

in

$-24.5$

$\begin{array}{lll}-13.5 & -16.5 & -14.5\end{array}$

$-14.5$

$\begin{array}{llll}-1.5 & 0.75 \text { si } & -.5 & \text { iN }\end{array}$

$-.5$

$1.75 p$ i 8.25

$14.250: 15.75 \rho \mathrm{si} 14.5$

$\frac{7.25}{4.5}$

(signoff)Calibrated BY:

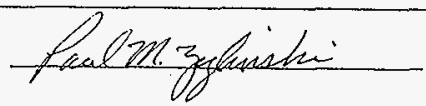

Date: 2 in. 57

Location: $101 \mathrm{AZ}$ cooling, water tower (sw) in Pump suction

comments Compound gauge 
Tag: $P_{1} \cdot A Z 101 \varepsilon \omega P \cdot 1 B Z$ Z\&ID: LOOP:

Manufacturer: Mulaniel Controts modei no.: Satety Gauge serial no: 28.000663 MTE Instr. No: $817: 35 \cdot 40 \cdot 011$ Due Date: $5 \cdot 22 \cdot 97$

\begin{tabular}{|c|c|c|c|c|c|c|c|}
\hline \multirow{2}{*}{\multicolumn{2}{|c|}{$\begin{array}{l}\text { Input Range } 0-100 \\
\text { Units: ps. }\end{array}$}} & \multicolumn{6}{|c|}{ Tolerance $\pm 5 \%$} \\
\hline & & & & & & & \\
\hline Input & Output & Lo: & $\mathrm{ri}$ : & As & 5. Found & In/out & As Left \\
\hline 0 & 0 & -5 & 5 & & 0 & IN & 0 \\
\hline 25 & 25 & 20 & 30 & & 25 & IN & 25 \\
\hline 50 & 50 & 45 & .55 & & 50 & $\therefore$ IN & 50 \\
\hline 75 . & 75 & 70 & 80 & . & 75 & ins & 75 \\
\hline 100 & 100 & 95 & 105 & & 99.5 & uN & 99.5 \\
\hline
\end{tabular}

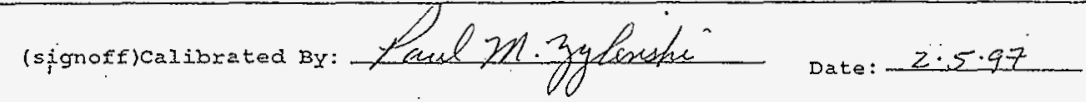
Location: Lor Az Coding water touser (EwP IB - Pump Discharge) 
Tag: PI.AZ102EWP-|A|

PEID:

LOOP:

HNF-SD-W030-TD-003,

REV. 0, PAGE 296

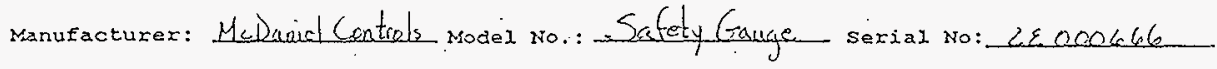
MTE Instr. No: $817 \cdot 35 \cdot 40 \cdot 030$ Due Date: $7 \cdot 31 \cdot 97$

Input Range $0-30 " \mathrm{Hg}$ Vac
Units: lig Vac
Input psi
output

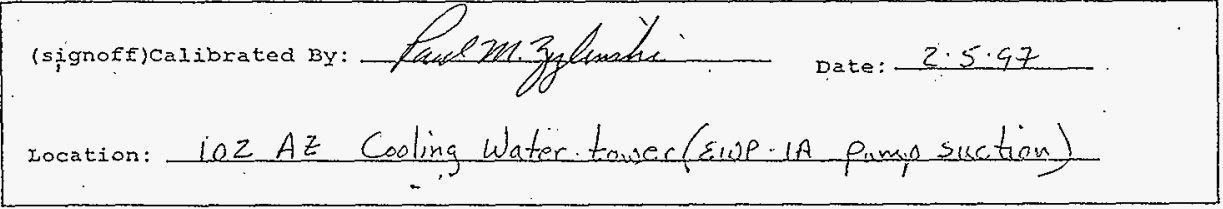

comments Compound gauge 
Tag: $P|\cdot A z 102 E W P \cdot| A 2$

P\&ID :

LOOP:

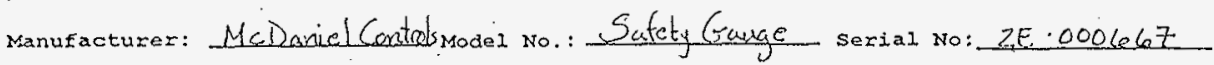
MTE Instr. No: $817 \cdot 35 \cdot 40 \cdot 011$ Due Date: $5 \cdot 22 \cdot 97$

$\begin{array}{lc}\text { Input Range } & 0- \\ \text { units: } & \text { psi } \\ \text { Input. } & \text { output } \\ 0 & 0 \\ 15 & 15 \\ 30 & 30 \\ 45 & 45 \\ 60 & 60\end{array}$

Tolerance $\pm 5 \%$

Input Rarge $0-60$
60.60

tput

5

30

60

\begin{tabular}{ccccc} 
Lo: & hi: & As Found & In/out & As Left \\
-3 & .2 & 0.5 & N & 0.5 \\
\hline 12 & 18 & 14.5 & IN & 14.5 \\
\hline 27 & 33 & 30.0 & IN & 30.0 \\
\hline 42 & 48 & 45.0 & 10 & 45.0 \\
\hline 57 & 63 & 60.0 & iN & 60.0 \\
\hline
\end{tabular}

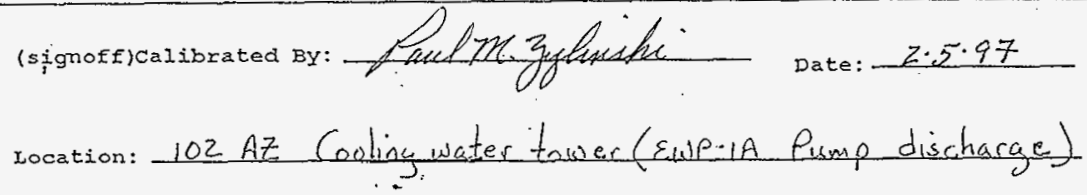

Comments 
rag: $P_{1} \cdot A Z 102 E \omega P \cdot|B|$

P\&ID :

HNF-SD-W030-TD-003,

REV. O, PAGE 298

Loo: :

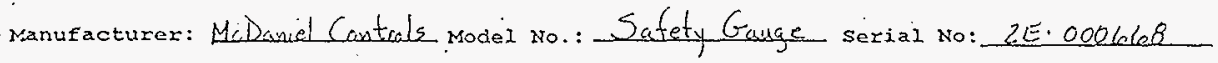
MrE Instx. No: $817 \cdot 35 \cdot 40 \cdot 030$ Due Date: $7 \cdot 31 \cdot 97$

Input Range $\begin{aligned} & 0.30 \mathrm{im} \cdot \mathrm{Hg} \mathrm{Vac} \\ & 0-15 \text { psi }\end{aligned}$

Tolexance $\pm 5 \%$

Units: in Hlg Vac

Input output

$-25 \mathrm{inhy}-25 \mathrm{inHg}$

Io:

A.s. Found

In/out

As Left

-15 in $\mathrm{Hg} \quad-15$ in $\mathrm{Hg}$

0

0

7.5 psi 7.5 p.si

$15 \mathrm{psi} \quad 15 \mathrm{psi}$

$-23.5 \quad-26.5$

$-24.5$

IN

$-24.5$

$-13.5 \quad 16.5$

$-14: 5$

IN. -14.5

$-1.5 \quad 0.75,0 ;$

0

IN

0

$6.75 p_{i} 8.25 p_{i} \quad 7.25 \quad$ is $\quad 7.25$

14. 25 pri 15.75 ps:

14.75 iN

14.75

(signoff)Calibrated BY:

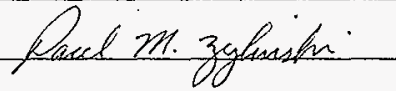

Date: $2 \cdot 5 \cdot 97$

Location: $\ln A z$ Conling woter tower (Ewp-1B pump suction)

Comments Compound Gauge. 
Tag: $P_{1} \cdot A Z_{102 E W}-1 B 2$

P\&ID:

LOOP:

Manufacturex: MaDraiel Controls Model vo.: Safety Gauge serial so: $2 E .000669$ Mre Instr. No: $817 \cdot 35 \cdot 40 \cdot 011$ Due Date: $5 \cdot 22 \cdot 97$

\begin{tabular}{|c|c|}
\hline Units: & \\
\hline $\begin{array}{c}\text { Input } \\
0\end{array}$ & $\begin{array}{c}\text { Output } \\
0\end{array}$ \\
\hline 15 & 15 \\
\hline 30 & 30 \\
\hline 45 & 45 \\
\hline 60 & 60 \\
\hline
\end{tabular}

$$
\text { rolerance } \pm 5 \%
$$

Units: $\rho$ si

Input. output INO: $E i$ :

As Found

In/out As Left

\begin{tabular}{ccccc}
-3 & 3 & 0 & 10 & 0 \\
\hline 12 & 18 & 14.5 & 10 & 14.5 \\
\hline 27 & 33 & 29.5 & 10 & 29.5 \\
\hline 42 & 48 & 44.5 & 10 & 44.5 \\
\hline 57 & 63 & 60.0 & 10 & 60.0 \\
\hline
\end{tabular}

(siggnofficalibrated BY:

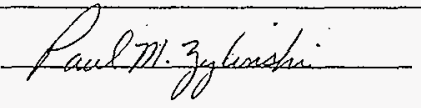

Date: $-2 \cdot 5 \cdot 97$

Iocation: $102 \mathrm{Az}$ Cooling Water tower (EwP.1B pump discharge)

Comments. 
$6 / 6 / 96$

F-030 CALIBRATION DATA SHEET

HNF-SD-W030-TD-003,

REV. 0 , PAGE 300

Tag: Pl. AZ 102WS-Z

R\&ID:

LOO:

Manufacturer: ASHCROET Model No .:-0-60 serial No: 2E. 000664

MTE Instr. No: $-817 \cdot 35 \cdot 48 \cdot 011$

Due Date: $\quad 5 \cdot 22 \cdot 97$

Input Range $0-60$

Tolerance $5 \%$

units: Psi

$\begin{array}{cc}\text { Input } & \text { Output } \\ 0 & 0 \\ 15 & 15 \\ 30 & 30 \\ 45 & 45 \\ 60 & 60\end{array}$

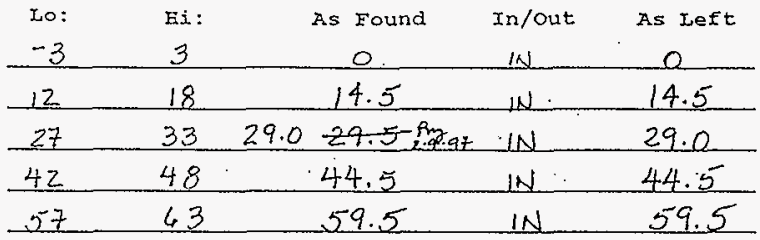

(signoff)Calibrated BY:

Mm. Jossiche

Date: $2 \cdot 4 \cdot 97$.

Location: 102. AZ Cooling water tower

Comments 
HNF-SD-W030-TD-003, REV. O, PAGE 301

Tag: P1. Azlozws - 1
I.0:
PEXD:

Manufacturex: A.5HCRDFT Model No.: $0-60$ serial No:2E-000665 MTE Instr. No: $817 \cdot 35 \cdot 40 \cdot 0 / 1$ Due Date: $5 \cdot 22 \cdot 97$

Input Range $0-60$

Units: PSi

Input output

$0 \quad 0$

$15 \quad 15$

$30 \quad 30$

45.45

60.60
Tolerance $5 \%$

\begin{tabular}{ccccc} 
Lo: & Ei: & As Found & In/out & As Left \\
-3 & 3 & +.5 & IN & +.5 \\
\hline 12 & 18 & 15.0 & IN & 15.0 \\
\hline 27 & 33 & 29.5 & IN & 29.5 \\
\hline 42 & 48 & .44 .0 & iN & 44.0 \\
\hline 57 & 63 & 59.5 & NN & 59.5
\end{tabular}

(signoff)Calibrated By:

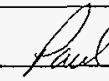

Date: $2: 4 \cdot 97$

rocation: 102-Az Cooling water tower

Comments 


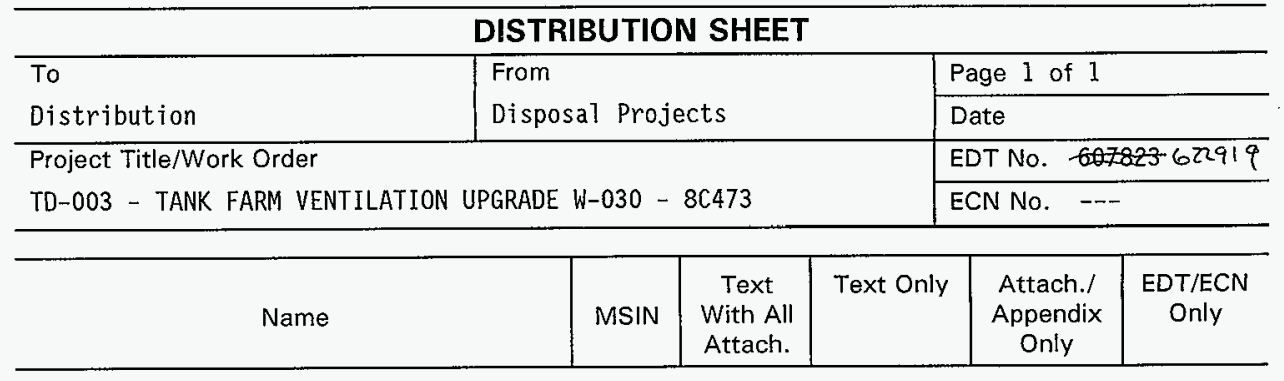

US Department of Enerqy

Richland Operations office

C. R. Pacheco

S7-54 $\quad x$

Fluor Daniel Northwest Company

D. P. Devine

G3-12

H. M. Chafin

R3-25

$x$

Lockheed Martin Hanford Company

G. P. Hopkins

S. U. Zaman

S. R. Pierce

W. D. Winkelman

W. M. Harty

J. R. Kriskovich

$\begin{array}{rrr}\begin{array}{l}5-03 \\ \text { S5-12 }\end{array} & X \\ -55-05-12 & X & X \\ \text { R3-12 } & X \\ \text { S5-13 } & & X \\ \text { R1-56 } & & X\end{array}$

Numatec Hanford Company

K. A. Colosi (2 copies)

D. B. Cole

T. Choho

M. D. Gerken

R3-25

R3-25

R3-47

R3-38

$x$
$X$
$x$
$X$

B\&W Hanford Company

L. F. $\mathrm{Hill}$

N2-54

X

others

DPC/TFIC

W-030 Project Files

A3-94

R1-29

$x$
$x$ 